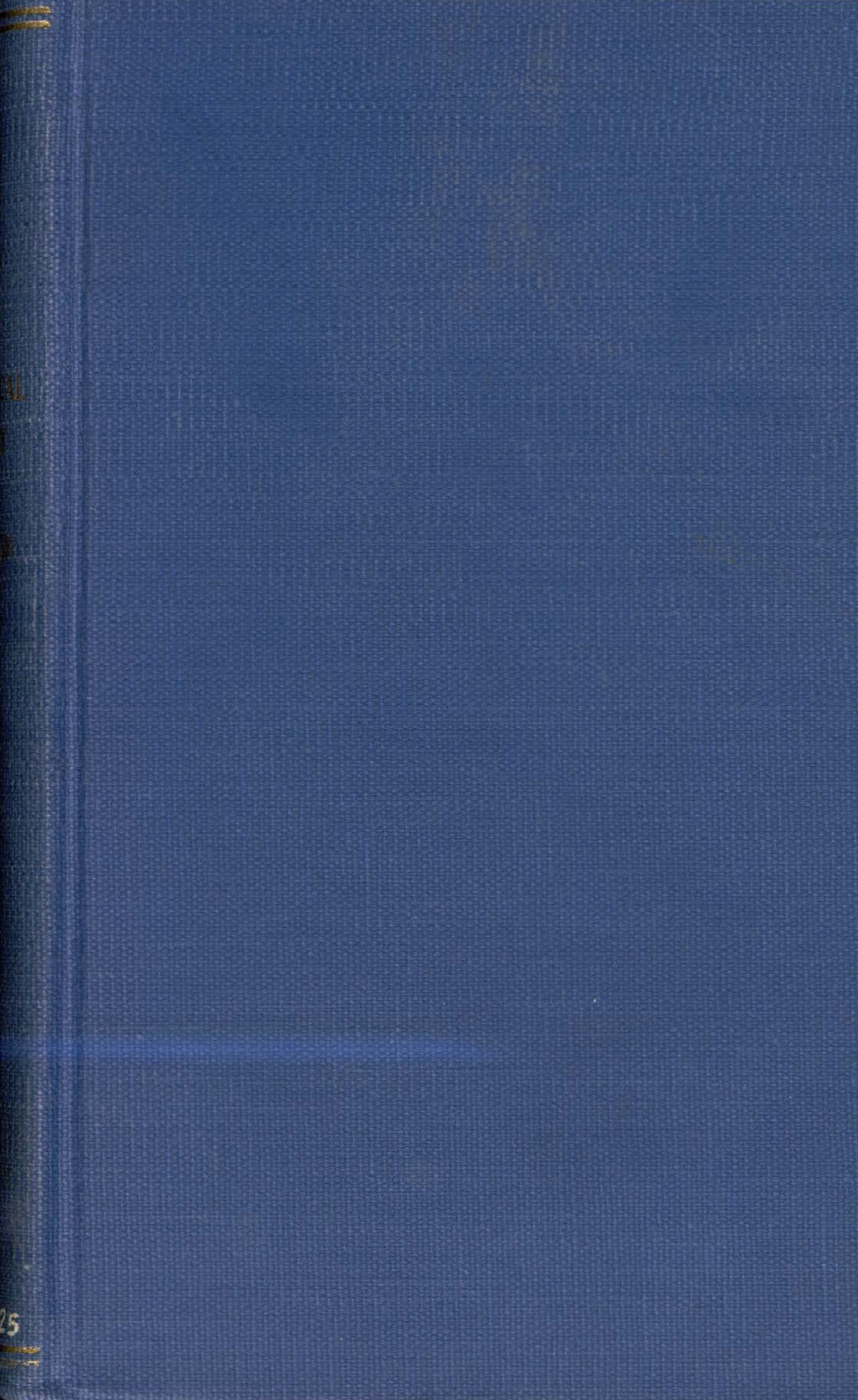







UNITED STATES DEPARTMENT OF THE INTERIOR

\section{MICROSCOPIC DETERMINATION OF THE ORE MINERALS}

\section{GEOLOGICAL SURVEY BULLETIN 825}

\section{U. S. AHISTAOHO}

WUESE, TEXAS 

UNITED STATES DEPARTMENT OF THE INTERIOR

Ray Lyman Wilbur, Secretary

GEOLOGICAL SURVEY

George Otis Smith, Director

\section{Bulletin 825}

$\overline{.60}$

\section{MICROSCOPIC DETERMINATION OF THE ORE MINERALS}

BY

M. N. SHORT

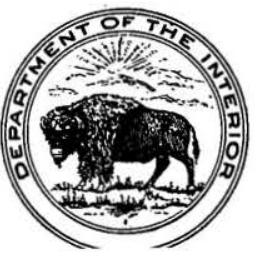

UNITED STATES

GOVERNMENT PRINTING OFFICE

WASHINGTON : 1931 



\section{CONTENTS}

Page

Preface

Part 1. Microscopic technique... 1

Introduction................... 1

The metallographic microscope._... 3

Mounting the specimen....... 7

Mounting in modeling wax

Mounting in brass boxes.

Warming oven

Polishing the specimen

Polishing process at the United States Geological Survey...... 10

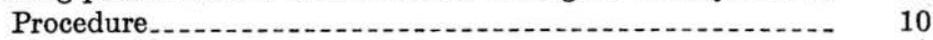

General remarks . . .

Impregnating the specimen

Polishing process at Harvard University

Polishing process at the University of Minnesota

Photography of polished sections...

Photomicrographic camera

Light filters. .

Exposure formula............... 26

Developing and printing

References............ 29

Part 2. Physical properties.

Color

Hardness.......

Examination of opaque minerals in polarized light_....... 39

Summary _._. 39

References...... 39

Apparatus........ 40

Details of the method...... 42

Methods of observation....... 45

Adjustment of the instrument.

Sources of error

Electroconductivity

Part 3. Etch reactions and determinative tables..... 50

Etch reactions.......... 50

Determinative table

Introduction..... 58

Index

Outline of table

Soft minerals

Hard minerals

Binnenthal minerals... 103

Discredited or doubtful minerals

Index of minerals grouped by elements...... 107 
Part 4. Microchemical methods................... 115

Introduction................ 115

Procedure and apparatus................... 116

Collecting the material .......... 118

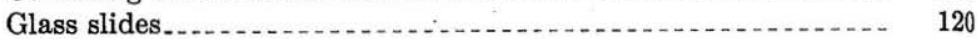

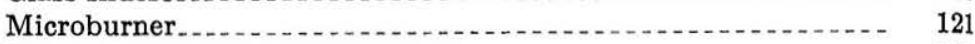

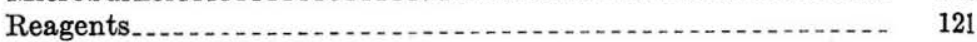

Capillary tubes.

Methods of applying tests............. 123

Sensitivity of microchemical tests _...

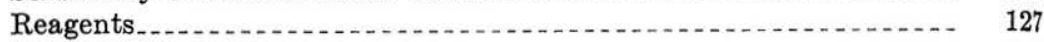

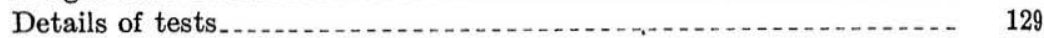

Zinc

Copper.

Nickel . .

Cobalt....

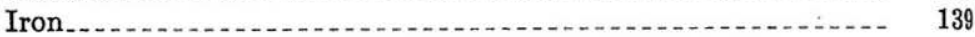

Mercury . . . .

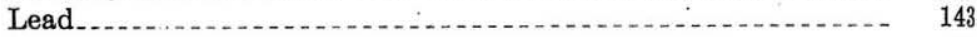

Silver....

Gold

Arsenic . . . . .

Antimony and bismuth.

Tin

Tellurium ....

Selenium

Manganese......... 168

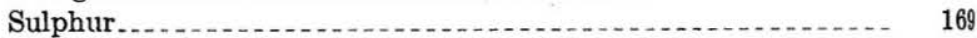

A systematic scheme of microchemical analysis............ 173

Specific tests for minerals . . . . .

Index

\section{ILLUSTRATIONS}

Plate 1. Photomicrographs of polished specimen of ore from Magma

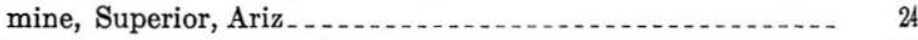

2. Microchemical tests for zinc.

3. Microchemical tests for copper............ 136

4. Microchemical tests for cobalt and nickel........... 136

5. Microchemical tests for mercury ........ 136

6. Microchemical tests for lead......

7. Microchemical tests for silver........... 152

8. Microchemical tests for gold.

9. Microchemical tests for arsenic and tellurium ......... 168

10. Microchemical tests for antimony ........... 168

11. Microchemical tests for bismuth and tin .................... 168 
FIGURE 1. Metallographic microscope with equipment for investigating

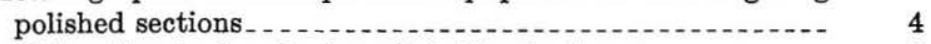

2. $a$, Prism illuminator; $b$, glass-disk illuminator....... 5

3. Improvised microscope camera

4. Leitz "Makam" camera attachment_................. 21

5. Bausch \& Lomb large metallographic camera. . . . 22

6. Comparison microscope _... 31

7. Talmage hardness machine

8. Microscope arranged for use with polarized reflected light_._- 41

9. Photomicrographs of ores taken in polarized reflected light_- $\quad 47$

10. Platinum wire for transferring drop of reagent to polished surface.................. 52

11. Enlarged view of needle for scraping polished surface .... 119

12. Improvised alcohol lamp .................... 121

13. Capillary tube .

14. Diagram showing Chamot's method I _........ 124

15. Glass-stoppered dropping bottle_.

16. Zine and copper mercuric thiocyanate crystals_........ 131 



\section{PREFACE}

The development of improved methods for the determination of the ore minerals and the collection of data on these minerals have occupied the attention of the writer for more than four years. The work has been done mostly in the laboratories of the United States Geological Survey except from September, 1927, to June, 1928, while he occupied the position of lecturer in economic geology in Harvard University and carried forward the work with the aid afforded by the resources of the Harvard Laboratory of Economic Geology.

It would be impossible to do full justice to all who have contributed to the completion of the present work. The writer wishes to acknowledge his especial indebtedness to his former teacher, Prof. L. C. Graton, who first aroused his interest in the subject and who has on many occasions given his counsel on the various problems encountered. Professor Graton also lent to the writer Murdoch's original suite of ore minerals, which is deposited in the Harvard Laboratory of Economic Geology.

Much assistance was rendered by the writer's former students, Drs. H. J. Fraser and H. A. Powers, who contributed some valuable original data as well as advice and criticism.

Dr. William F. Foshag, curator of mineralogy of the United States National Museum, has at all times helped the writer in his endeavors to procure authentic specimens of ore minerals. Practically all of the writer's suite of specimens were obtained from the National Museum. The Roebling and Canfield collections of minerals, recently acquired by the Museum, have proved valuable sources of rare minerals.

Dr. George A. Thiel, of the University of Minnesota, made available the results of his admirable research on the manganese minerals and gave generously of his time in checking the identity of the manganese minerals in the writer's collection.

Prof. Edward Sampson, of Princeton University, contributed to much of the recent improvements in the technique of polishing and more than anyone else is responsible for the perfection of methods using polarized light in the investigation of opaque minerals. Much of the information on the subject contained in this bulletin was taken from Professor Sampson's recent article.

Finally the writer desires to thank his colleagues of the Geological Survey for the assistance they have given him. George Steiger made possible several quantitative analyses of doubtful minerals. Thomas $\mathrm{S}$. Lovering read the manuscript and has given valuable advice and criticism. 



\title{
MICROSCOPIC DETERMINATION OF THE ORE MINERALS
}

\author{
By M. N. ShorT
}

\section{PART 1. MICROSCOPIC TECHNIQUE}

\section{INTRODUCTION}

The metallographic microscope is the most valuable instrument for identifying opaque minerals and for studying their mutual relationships. It plays the same part for the opaque minerals that the petrographic microscope does for transparent minerals. The metallographic microscope differs from the petrographic microscope in that it contains a reflector placed in the barrel of the microscope directly above the objective, and light is reflected vertically downward through the objective onto the specimen to be studied. The specimen or mineral is prepared for study by grinding and polishing a flat surface. It is then mounted so that the polished surface is parallel to the microscope stage, and the microscope is focused on the surface to be studied.

The identification of the opaque ore minerals in polished sections has made much progress since 1916, when Murdoch's pioneer researches were first published. ${ }^{1}$ The textbooks of Davy and Farnham, ${ }^{2}$ Schneiderhöhn, ${ }^{3}$ and Van der Veen ${ }^{4}$ have all contributed new ideas and methods of attack.

Not all the methods outlined in the texts cited have proved entirely satisfactory, and some discrimination is required in selecting from the methods described those which are of greatest value. The writer has made an effort to select those methods which, in his opinion, are most useful and which at the same time do not require elaborate and expensive apparatus. The scheme of identification adopted is based on the following observations:

\footnotetext{
${ }^{1}$ Murdoch, J., Microscopical determination of the opaque minerals, New York, 1916.

'Davy, W. M., and Farnham, C. M., Microscopic examination of the ore minerals, New York, 1920.

'Schneiderhőhn, Hans, Mikrokopischen Bestimmung und Untersuchung von Erzen, Berlin, 1922.

- Van der Veen, R. W., Mineragraphy and ore deposition, The Hague, 1925.
} 
1. By means of polarized light, minerals crystallizing in the isometric system can be separated from those crystallizing in the other five systems.

2. By means of a needle, minerals can be graded as to hardness? into two classes-hard, or those which can be scratched with dif. ficulty or not at all, and soft, or those which are easily scratched with a needle. The three grades of hardness described by Murdoch and by Davy and Farnham are accordingly modified by eliminating the "intermediate" class.

3. The systematic etching scheme described by Davy and Farn. ham, although far from perfect, is of great value in saving time. The etch tests are sufficient for the identification of some minerals, but for most minerals identifications based on this method must be checked by other methods.

4. Qualitative chemical tests on a microscopic scale constitute the most reliable method available for identifying an ore mineral. These tests require considerable time. Unless the investigator has a clue as to what elements are present, he must test for the different ele. ments in turn in a systematic way, analogous to the methods of ordinary qualitative analysis. The most effective and the quickest method of identifying a mineral is first to obtain all the data yieldel by methods 1 to 3 . This procedure will generally limit the designa. tion to a choice of half a dozen or less minerals. The final choic will be governed by definite microchemical tests for one or more suspected elements.

The necessity for more accurate data than are available in the literature was brought out during the present investigations. Micro. chemical tests made on Murdoch's original suite of ore minerals and on the suite of specimens selected by the writer from the United States National Museum showed that over 20 per cent of these minerals were mislabeled. The necessity for careful selectiol of specimens is thus manifest.

The present investigation by no means eliminates all the errors resulting from incorrectly designated specimens. Microchemical tests are qualitative and not quantitative and are thus incapable of distinguishing between different minerals having the same elements. The writer can not, at present, distinguish beegerite $\mathrm{Pb}_{6} \mathrm{Bi}_{2} \mathrm{~S}_{9}$; cosalite, $\mathrm{Pb}_{2} \mathrm{Bi}_{2} \mathrm{~S}_{5}$; and lillianite, $\mathrm{Pb}_{3} \mathrm{Bi}_{2} \mathrm{~S}_{6}$. The onl safe way of distinguishing between these minerals would be to obtail quantitative chemical analyses of selected specimens and try to ob tain distinctive criteria on polished sections of these specimens $b$ methods 1 to 3 outlined above. Usually but not always if suffices to obtain material from the type localities of the minerals With such material it is not necessary to make analyses, as th 
original identification of the minerals from these localities is usually based on quantitative analyses. This plan is especially useful on minerals that are confined to one or two localities. For example, a mineral from Rezbanya, Hungary, containing lead and bismuth and labeled "rezbanyite" is probably what it purports to be.

It should not be forgotten, however, that many published analyses, especially the earlier ones, were based on impure material. Before the reflecting microscope was applied to the study of ores many ore minerals were assumed to be pure because no impurities were visible in the hand specimen. Microscopic investigation has shown the error of such an assumption. Even apparently pure crystals are by no means always pure. The correct formula for bornite escaped determination for over a hundred years because the crystals of apparently pure bornite analyzed were not pure but were later shown to be intermixtures of bornite and chalcopyrite. It thus becomes evident that no ore mineral should be analyzed until it has first been examined microscopically and shown to be pure. In the descriptions of minerals given in this bulletin, data obtained from analyzed material will be accompanied by reference to the analyses.

A suitable designation for the study of the ore minerals under the metallographic microscope has not yet been suggested. The term "mineralography" proposed by Murdoch is obviously not suitable, as the study embraces only a certain group of minerals. The term "mineragraphy" proposed by Whitehead ${ }^{5}$ and used by Davy and Farnham and by Van der Veen is decidedly harsh and unpleasant. The term "chalcography" used by Schneiderhöhn is unsuitable, as it has already been applied to the art of engraving on copper. ${ }^{8}$ The writer prefers not to use any of the above terms and will retain the exact if cumbersome phrase " microscopic study of the ore minerals."

\section{THE METALLOGRAPHIC MICROSCOPE}

Any compound microscope can be converted into a metallographic microscope by means of a vertical illuminator, consisting of a short tube in which is mounted a reflector. An opening in the side of the tube admits light to the reflector. The light after impinging on the reflector is thrown vertically downward through the objective and strikes the polished surface of the mineral to be examined. The light is then reflected vertically upward from the polished surface, passes through the objective and behind or through the reflector, and after passing through the ocular reaches the eye.

' Whitehead, W. L., Notes on the technique of mineragraphy: Econ. Geology, vol. 12, p. $697,1917$.

- Webster's New International Dictionary. This confusion in terminology was first noted by Mr. E. E. Fairbanks in an unpublished paper. 
There are two types of vertical illuminator, the difference depend: ing on the kind of reflector used. The prism illuminator consists of a glass prism which occupies approximately one-half of the area of: the tube. This arrangement enables part of the rays reflected from the surface to pass behind the reflector. (See fig. 2.) The glass. disk illuminator consists of a thin transparent glass disk whici occupies practically the entire area of the tube. In both types the reflector is rotatable in the tube and is turned until the clearest image appears.

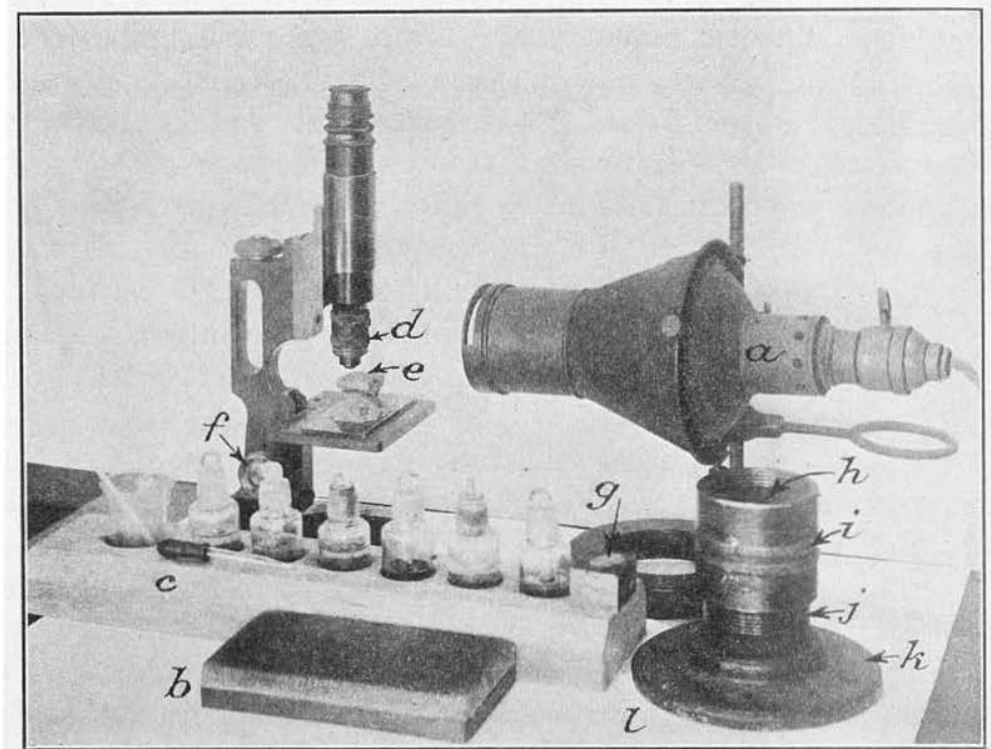

FIgUre 1.-Metallographic microscope with equipment for investigating polished sections. $a$, Spencer lamp, No. 374; $b$, rubbing block; $c$, reagents for etch tests; $d$, vertical illuminator; $e$, polished section on modeling wax: $f$, screw for racking stage up and down; $g$, polished section in brass mount; $h$, lead filling; $i$, sleeve; $j$, nipple; $k$, flange; $l$, mounting cup

The vertical illuminator screws on to the lower end of the microscope barrel, and the objective, in turn, screws on or is fastened br a spring clip to the lower end of the illuminator.

The glass-disk illuminator, also referred to as the plane-glas illuminator, gives a more even illumination than the prism illuminator, but only about half of the light striking the glass disk is re flected downward to the polished section; the rest of the light is absorbed by the disk or passes horizontally through it and is absorbed in the walls of the illuminator tube. The light impingine downward on the polished surface is reflected upward. On strik ing the plate-glass disk again, part of the upward-moving light i: reflected back toward the lamp and leaves the microscope. A smal proportion of the upward-moving light is absorbed by the dist 
itself, and the remainder gets through the disk and so reaches the eye. As a result of these losses only a small part, possibly less than one-fourth, of the original intensity of the light is available for microscopic observation.

The prism illuminator reflects downward practically all the light that impinges on the prism. However, only part of the light reflected by the polished section reaches the eye, as the prism itself occupies half of the section of the tube, and all that part of the light which, on its upward path, strikes the prism is reflected outside of the microscope and lost. Only that part of the upward-moving light that passes behind the illuminating prism reaches the eye. To get the maximum illumination the prism is turned so that the rays do not move exactly vertically downward but strike the surface
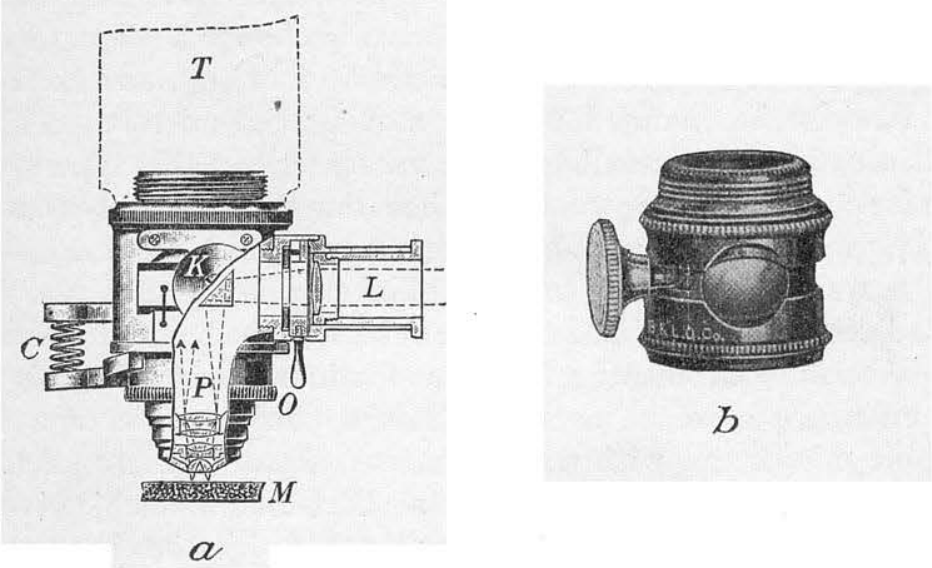

Figurn 2.- $a$, Prism illuminator. (Courtesy of E. Leitz, Inc.). $T$, microscope barrel ; $K$, prism; $L$, converging lens; $O$, short-mounted objective; $P$, bundle of rays passing upward behind prism; $M$, polished surface; $C$, spring clip holding objective to illuminator barrel. b, Glass-disk illuminator. (Courtesy of Bausch \& Lomb Optical Co.)

with a slight inclination from the vertical and in turn are reflected with just enough inclination from the vertical to pass by the prism.

Van der Veen ${ }^{7}$ compares the two types of illuminator as follows:

The specific intensity of the illumination by the prism illuminator is higher than that of the glass [disk] illum nator, because the bundle of rays, although divided into two parts, keeps its original intensity. In the plane-glass type every ray is reduced in intensity while the whole bundle remains intact. For this reason, the resolving porver of the plane-glass illuminator is much higher than that of the prism illuminator. Therefore, the former is especially desirable for high magnification.

The writer prefers the prism type of illuminator for work with daylight and with ordinary artificial illumination (using an ordinary incandescent electric bulb and a daylight glass screen). This

${ }^{7}$ Van der Veen, R. W., op. cit., p. 3. 
is satisfactory for use with the 16-millimeter and 4-millimeter ob. jectives. With objectives of higher magnification than the 4-milli. meter lens and lower magnification than the 16-millimeter lens and for microphotography the glass-disk illuminator is preferable. Strong artificial illumination is required for the higher magnifica. tions. The writer finds the 6-volt 108-watt tungsten-ribbon Mazda lamp the most satisfactory. The arc lamp gives an intense light but one which varies in intensity.

Figure 1 shows the most common type of metallographic micro. scope. The stage can be moved vertically by means of a rack and pinion but is not rotatable. The microscope barrel with attached illuminator and objective remains fixed, and the polished surface is brought into focus by racking the stage up or down. The advantage of this arrangement is that the light source does not need to be shifted in position when the objective is changed. The distance of the polished surface above the glass slide that supports the specimen can likewise be changed without moving the microscope barrel or light source. The metallographic microscope of this type is entirely satisfactory for most work but has the disadvantage that it can not be used in investigations requiring polarized light.

The writer prefers a petrographic microscope for use with rẹ. flected light. The illuminator can be attached and detached in a few seconds by means of a ring that fits in a clamp. In this manner the microscope can be changed from a petrographic to a metallo. graphic microscope with a minimum of effort. Nearly all the work with reflected light is done with a short-mounted objective of 16 . millimeter focal length. Consequently, the objective is screwed into the vertical illuminator and not detached from it when the illuminator is removed from the microscope.

The 16-millimeter objective is the best all-round lens for metallographic work for the following reasons:

1. It gives the most satisfactory field of view for the greatest number of mineral intergrowths - that is, the size of mineral grains in the greatest number of specimens is such that they are best ob. served with that lens.

2. It combines clearness of vision with depth of focus and freedom from boundary shadows. (See pl. 1.)

3. It is the best lens for use with polarized light. (See p. 42.)

Objectives of lower magnification than the 16-millimeter lens are more frequently used than those of higher magnification. The 24 . millimeter and 32-millimeter lenses are the ones most frequently used, These two lenses do not have to be designed for use without the cover glass and do not require special short mounts; the 24 and 32 millimeter petrographic objectives are entirely satisfactory for metallographic work. 
If the mineral grains are so large that they require the use of an objective of still lower magnification, it is best to dispense with the use of the metallographic microscope altogether and view the polished section under the binocular microscope, using oblique light. By tilting the surface at the correct angle, a mirrorlike reflection is obtained which is similar to that produced by vertical illumination.

The 8-millimeter and 4-millimeter short-mounted objectives and more rarely the short-mounted 1.9-millimeter oil-immersion lens are occasionally used for higher magnifications, but the difficulty of obtaining a flat surface on a polished mineral specimen hinders the use of the oil-immersion lens. Fortunately the size of grain in mineral intergrowths is rarely so small that the oil-immersion lens is required.

The rotatable stage and tube analyzer of the petrographic microscope are necessary when polished sections are examined in polarized light.

For ordinary microscopic work, where the comparison microscope is not required, daylight furnishes satisfactory illumination. With some experience, the eye readily adjusts itself to changing strength of daylight. A north exposure or one shielded from direct sunlight is required. If trees, buildings, or other objects of any considerable size are in front of the microscope, the images of these objects are apt to be reflected on the surface of the mineral under the microscope. If an uninterrupted view can not be obtained, artificial illumination is required. The writer used a Spencer microscope lamp No. 374, made by the Spencer Lens Co., Buffalo, N. Y., equipped with a 115-volt 100watt Mazda bulb, a condenser, and a "daylight" color screen.

\section{MOUNTING THE SPECIMEN}

Mounting in modeling wax.-The polished specimen is mounted by being pressed into a lump of modeling wax on a glass slide. A convenient size for the slide is $31 / 2$ by $11 / 2$ inches by $1 / 8$ inch. The polished surface is, of course, uppermost and must be parallel to the glass slide. A number of leveling devices are on the market, but none of them are as convenient as the home-made mounting cup made of 2-inch pipe fittings shown in Figure 1, which is similar to one illustrated by Davy and Farnham. ${ }^{8}$

This consists of a 2-inch nipple 3 inches long filled with lead, a sleeve $2 \frac{1}{2}$ inches long, and a flange. The upper surface of the nipple with its lead filling and the upper edge of the sleeve are machined so as to be at right angles with the length. The sleeve is rotated so that its upper edge is a convenient distance above

\footnotetext{
${ }^{8}$ Davy, W. M., and Farnham, C. M., op. cit., p. 5.
} 
the lead surface. The distance selected determines the distance be. tween the polished surface and the upper surface of the slide. The section is pressed down by hand into the wax, the polished surface being approximately parallel to the glass slide; then the slide with wax and section is turned upside down, and the polished surface is lowered till it comes into contact with the lead surface of the cup. The slide is then pushed downward until it rests on the sleeve. The advantage of this leveling device is that the height of the polished surface above the slide can be adjusted at will.

Mounting in brass boxes.-A much more effective method of mounting than the temporary emplacement in modeling wax is to cement the polished chip in a section of rectangular brass tubing by means of sealing wax. The polished sections thus mounted are easier to handle, and tiny fragments or fragile chips that could not be held steadily by hand or that would break to pieces during the polishing process are firmly gripped in the cement, so that the grinding can be continued to any required degree. A flatter surface is obtained when the chip is mounted in the brass box than can be obtained on an un. mounted chip, as the entire area of the box acts as a support for tho specimen during grinding and polishing. In the United States Geo. logical Survey laboratory polished sections that are to be examined once and then discarded are polished unmounted, and those that are to be retained permanently are mounted in brass.

A rough flat surface is first ground on one side of the specimen before cementing it in the brass tube. After the specimen is mounted, the grinding is continued and is followed by the polishing process. If desired, however, the specimen can be completely ground and polished before mounting in brass.

The rectangular tubing used measures $11 / 2$ by $11 / 8$ inches outside dimensions in cross section and is cut to $7 / 8$-inch lengths. These tubes were furnished already cut by the American Brass Co., Waterbury, Conn., and cost approximately 3 cents each. Before the specimen is mounted, two conical indentations are punched in each of the longer sides of the tube to prevent the mounting medium from slipping in the brass. These indentations are driven in with a steel center punch, and to prevent inward bulging of the tubing when they are driven, it is placed over a steel bar which has a slot of triangular cross section cut on one face at right angles to the length of the bar.

Before the sealing wax is poured the number and other designations of the specimen are stamped on the shorter side of the brass tubing by means of a hammer and $\frac{3}{32}$-inch dies. The tube is supported while being stamped by a steel bar without the triangular slot. 
When a specimen is to be mounted in sealing wax both the specimen and the tube must be warmed to approximately the temperature of the molten wax; otherwise the sealing wax will shrink away from the walls of the tube in cooling. When the tube is warmed before pouring the sealing wax, the brass shrinks more than the wax and thus fits tightly to it.

The warming 'can be done on an electric hot plate but is most conveniently done in a warming oven. (See p. 10.) A steel plate $91 / 2$ by 10 inches by $1 / 4$ inch with one machined surface supports the specimens and their brass mounts. A sheet of cardboard is placed on the steel plate, and the sections are placed face down on it. The brass tubes are placed over the sections in such a way as to center each section in its mount. The steel plate is then placed in the oven or on the hot plate until a temperature on its upper surface of approximately $100^{\circ} \mathrm{C}$. is attained. The steel plate is then withdrawn, and the molten sealing wax is poured into the brass tubes until it reaches a level just above the indentations. About 30 specimens can be mounted in one pouring of sealing wax. When cool, the cardboard is easily removed from the mounts.

Sealing wax tends to flow during hot weather, and it has been found advisable to give the wax a backing of plaster of paris. The section is placed face down, and plaster of paris is poured until it almost fills the tube, about $1 / 8$ inch of clearance being left in order that the mount will rest on its brass edges when righted.

A sealing wax made of 120 grams of flaked shellac, 90 grams of resin, and 45 cubic centimeters of turpentine has been found entirely satisfactory and is superior to those on the market in that it is free from gritty filler. The ingredients are placed in a "granite-ware" saucepan about $5 \frac{1}{2}$ inches in diameter and $2 \frac{1}{2}$ inches deep, and the pan is heated until vigorous bubbling sets in, the mixture being stirred from time to time by means of a tablespoon. When the ingredients are thoroughly mixed, the pan is removed from the heat source and the mixture stirred until it ceases to bubble. Bubbles on the surface of the mount-that is, adjacent to the polished surfaceare objectionable, as they tend to collect grit during the subsequent grinding and polishing. Sections mounted as described above and kept away from the sun or other sources of heat will last several years without any appreciable deterioration of the sealing wax. The writer has sections that have been mounted five years, most of which are free from checking on the surface of the wax and others show it only slightly. Experience at Harvard has shown that eventually the sealing wax will disintegrate and the specimen will have to be remounted. 
Portland cement has been suggested as a mounting medium, ${ }^{9}$ but experience has shown that it is almost impossible to prevent particles of grit from breaking from the surface and scratching the softer minerals.

Undoubtedly the best mounting medium yet discovered is solid bakelite. This requires a hydraulic press with heating units, and as this apparatus costs at least $\$ 500$, it will probably not come into general use. This method has been developed at Harvard University but has not yet been described.

Warming oven.-In the Geological Survey laboratory the warm. ing is done in an oven equipped with electric heating coils. The oven measures 11 by 11 by $14 \frac{1}{2}$ inches and was manufactured by the Central Scientific Co., Chicago, Ill. By means of a thermostat a constant temperature is maintained. The temperature range is from $70^{\circ}$ to $115^{\circ} \mathrm{C}$.

A simple home-made oven will give good service. The design of this oven was suggested by Dr. Edwin P. Cox, formerly of the University of Oregon. It is a box constructed of metal and lined with asbestos to prevent the escape of heat. The box measures $161 / 2$ by $121 / 2$ by $121 / 2$ inches, outside dimensions. Heating is accomplished by six 75-watt electric bulbs arranged in parallel. Each bulb can be turned on or off by screwing it in or out in its socket. Such an oven constructed at Harvard University gave a temperature after two hours of $40^{\circ} \mathrm{C}$. with one bulb burning and $120^{\circ} \mathrm{C}$. with all six burning. The temperature maintained was very constant. Intermediate temperatures were obtained by varying the number of bulbs in the circuit. This oven serves very well in impregnating a porous specimen with bakelite, as described on page 15 .

\section{POLISHING THE SPECIMEN}

\section{POLISHING PROCESS AT THE GEOLOGICAL SURVEY}

Procedure.-The procedure outlined below is the one used in the petrographic laboratory of the Geological Survey for grinding and polishing unmounted specimens:

A. Removal of chip from hand specimen by chisel or with diamond saw.

B. Grinding of flat surface with 120 carborundum. Used only on hard ores; omitted on soft ores.

C. Grinding with $\mathrm{F}$ carborundum. This is the first grinding with soft ores and the second grinding with hard ores.

D. Grinding with 600 carborundum.

E. Polishing on muslin-covered lap with 65 alundum.

$F_{1}$. Polishing on billiard cloth with magnesia oxide or black magnetic rouge Omitted for some hard ores.

$\mathrm{F}_{2}$. Polishing on muslin-covered lap with chromic oxide or rouge. Used in place of $F_{1}$ on some hard ores. Omitted for most ores.

Short, M. N., Preparation of polished sections of ores : Econ. Geology, vol. 21, p. 658
} 
A. The desired fragment of the specimen is first chipped off by means of a cold chisel. Davy and Farnham ${ }^{10}$ advocated the use of a diamond saw, which consists of a steel disk with diamonds mounted in its periphery. Although this offers decided advantages, especially when a definite section through the specimen is required, its use is best avoided when possible because of its rapid wear when sawing hard minerals. A simple diamond saw, consisting of a thin copper disk which is fed with diamond dust, is described by Vanderwilt. ${ }^{11}$

A satisfactory surface can be prepared in a surprisingly short time by grinding on a horizontal lap with carborundum powder. Ordinarily the surface prepared should not exceed 1 square inch in area.

B. If the specimen consists of a large proportion of hard minerals, such as pyrite, it is best to do the first rough grinding with 120 carborundum. This is continued until a surface of sufficient area is obtained. The specimen is then removed and carefully washed to remove adhering carborundum grit. The hands should likewise be cleaned carefully to prevent coarse grit from getting into the succeeding finer grinding material. If the specimen consists mostly of softer minerals and contains only a minor proportion of pyrite or other hard minerals, it is best to begin the grinding process with $\mathrm{F}$ carborundum, as the coarser grades tend to tear deep holes and gashes in the softer minerals. A horizontal steel lap 12 inches in diameter is used. Its speed is 1,150 revolutions a minute.

After grinding the first surface, corners and projections of the specimen should be ground off in order to avoid excessive grooving of the surface by fragments breaking loose during the succeeding stages. It is advisable to bevel back the edge of the surface at least $60^{\circ}$ from the face, especially on specimens that are to be mounted in brass, as the mounting medium is more or less viscous and would have greater difficulty in flowing under a flatter beveled edge. The beveling is best done by holding the chip against the side of a rapidly rotating carborundum or emery wheel. If such a wheel is not available it can be done on the horizontal lap.

C. The section is then ground on a horizontal steel lap with $\mathrm{F}$ carborundum. The pits and scratches made by the coarser abrasive are removed, and a new series of pits and scratches, shallower than the first set, are made. Usually a minute suffices for this step. The speed of this lap is likewise 1,150 revolutions a minute.

D. The hands and specimens are carefully washed to remove any adhering $\mathrm{F}$ carborundum. The final grinding is done on a horizontal steel lap with 600 carborundum. The pits and scratches made by

${ }^{10}$ Davy, W. M., and Farnham, C. M., op. cit., p. 2.

"Vanderwilt, J. W., A simple diamond saw; Econ. Geology, vol. 25, p. 222, 1930. 
the F carborundum are ground out, and a still narrower and shal. lower series are made. Thirty seconds usually suffices for this stage of the grinding process. The section is then ready for polishing. The speed of this lap is likewise 1,150 revolutions a minute.

E. In the first stage of the polishing process 65 alundum is used on a lap covered with muslin or fine-textured linen. The linen is more expensive but requires changing less often because of its greater resistance to abrasion. The lap is 6 inches in diameter and its speed is 1,150 revolutions a minute. The cloth is first wet, then stretched tightly over the surface of the lap by means of a hoop around the periphery or by means of strong elastic bands held in place by a groove around the periphery. The amount of time required for polishing with $65 \mathrm{~F}$ alundum varies with the specimen. It is best to polish for one minute at a time, observing the effect under the micro. scope at the end of each step. When no further improvement is noted, the section is ready for the next stage. Ordinarily the $65 \mathrm{~F}$ alundum accomplishes all it can do in three minutes. If pits remain at the end of that time it is best to take them out on the chrome or rouge wheel. With some crumbly or very soft minerals the alundum keeps making new pits as fast as it removes the old ones. Experience is the best guide in determining when to discontinue polishing with $65 \mathrm{~F}$ alundum.

$\mathbf{F}_{1}$. The final polishing is accomplished by means of either mag. nesia or "black magnetic rouge" on a horizontal lap covered with billiard cloth. The trade name of this cloth is Samson cloth, and it is supplied by J. Magann \& Co., 78 Washington Street, Boston. Magnesium oxide or magnesia is effective in polishing pyrite and minerals of intermediate hardness. It also gives a good polish to softer minerals but tends to form more scratches than black mag. netic rouge. Magnesia is mixed with about 2 parts by volume of water and blown out of a wash bottle. Credit for discovering the value of magnesia as a polishing agent is due to Dr. Hans Schneiderhöhn, of the Technische Hochschule, Aachen, Germany. He advises keeping the bottle tightly stoppered, as the magnesia tends to absorb carbon dioxide from the air, forming magnesium carbonate, which scratches the softer minerals. Magnesium oxide actually has a greater hardness than the carbonate but breaks down in polishing to an exceedingly fine state of subdivision. Magnesia will remove fine pits as well as scratches from pyrite. Five minutes or less usually suffices to give a perfect polish to pyrite. Additional time may be advisable for polishing some minerals such as marcasite, which are prismatic in habit and more or less porous. The writer has spent as much as 15 minutes to good advantage. Magnesia is ineffective in polishing specularite and some other minerals of equal or greater 
hardness; for these minerals chromic oxide or rouge is more satisfactory.

"Black magnetic rouge" is a polishing powder manufactured by Binney \& Smith, 41 East Forty-second Street, New York. Its use was recommended to the Geological Survey by Prof. Alan M. Bateman. According to the manufacturers it is obtained by a patented process of precipitation and consists of 99 per cent $\mathrm{Fe}_{2} \mathrm{O}_{3}$. In general it has the same effect as magnesia, but it does not cut as fast. It is the best medium for polishing soft minerals, such as galena and chalcocite. It is more stable than magnesia and does not deteriorate when exposed to the air. It is mixed with a small proportion of water and applied to the cloth by means of a small paint brush. When used wet it will remove practically all the scratches, but in spite of care to prevent contamination by extraneous grit, a few scratches will persist, however long the polishing is continued. If the section is to be photographed, these last scratches can usually be removed by the following procedure, which was developed by F. S. Reed :

Apply moist magnetic rouge to the rotating lap, and press the specimen very lightly against it, giving the specimen a slow rotation. Continue until the lap is entirely dry. Some skill and experience are required in manipulation. If polishing is continued too long after the lap is dry the specimen tends to overheat. If polishing is stopped too soon the magnetic rouge tends to gum up and adhere to the specimen.

$\mathrm{F}_{2}$. Polishing on a billiard cloth with magnesia or black magnetic rouge as above described is effective for by far the greater number of specimens. It fails, however, to give a good final polish to very hard minerals, such as specularite, chromite, franklinite, and the hard manganese oxides. For this purpose fine floated, chromic oxide or rouge on a muslin or linen covered lap is recommended in place of magnesia as the final polishing agent. Neither fine floated chromic oxide nor fine floated rouge is available in the market, but both products are prepared in the Geological Survey laboratory by grinding in a small ball mill for 100 hours. The batch is removed from the ball mill and dumped into a tall cylindrical measuring jar 7.2 centimeters in diameter and 56 centimeters tall. Enough water is added to nearly fill the jar. The contents are then well shaken and allowed to stand 10 minutes. The top 15 centimeters of the contents of the jar is withdrawn by means of a siphon, the end of which should start at the top and be lowered slowly so as not to disturb the lower sludge. The material drawn off is placed in a 2.5 -liter bottle. The measuring jar is again nearly filled with water and thoroughly shaken and allowed to stand 10 minutes. The top 15 centimeters is again 
siphoned off into the bottle containing the sludge already removed. This process can be repeated until the 2.5 -liter bottle is filled. The bottle is allowed to stand over night, and the sludge settles to the bottom. The excess of clear water is siphoned off, and the sludge is ready for use.

The effect produced by rouge is practically identical with that produced by chromic oxide,but the chromic oxide is somewhat faster in its polishing action. Both powders are very effective in polishing quartz, glass, and the silicate minerals.

General remarks.-The average time for grinding and polishing an unmounted specimen by the process above described is from 12 to 15 minutes. If the specimen is mounted in brass an additional 10 minutes is required. The mounting should be done after the first flat surface is ground-in other words, between steps B and C. If the first surface is ground with $\mathbf{F}$ carborundum it should be mounted and then again ground with $\mathrm{F}$ carborundum until all edges of the brass tube rest evenly on the steel lap and the entire surface of the sealing wax has come into contact with the abrasive. The upper edges and corners of the brass mount should be beveled off after this stage of grinding is complete, in order to prevent the sharp edges of the brass from tearing the polishing cloth. The bevel should make an angle of about $45^{\circ}$ with the sides of the tube. Mounting in brass slows down the polishing somewhat but gives a more nearly plane surface to the specimen and does not affect adversely the quality of the polish.

The following table summarizes the characteristics of the abrasives as observed under the microscope. The size was measured by means of a micrometer ocular.

Characteristics of abrasives used in polishing mineral specimens

\begin{tabular}{|c|c|c|c|}
\hline \multirow[b]{2}{*}{ Abrasive } & \multicolumn{2}{|c|}{ Size (millimeters) } & \multirow[b]{2}{*}{ Characteristics } \\
\hline & $\begin{array}{c}\text { Largest } \\
\text { grain } \\
\text { observed }\end{array}$ & $\begin{array}{c}\text { Most of the } \\
\text { material }\end{array}$ & \\
\hline $\begin{array}{l}120 \text { carborundum } \\
\text { F carborundum } \\
600 \text { carborundum }\end{array}$ & \multirow{2}{*}{$\begin{array}{l}0.291 \\
.26 \\
.135 \\
.075\end{array}$} & \multirow{2}{*}{$\begin{array}{l}0.156 \\
.052-.104 \\
.02-.04 \\
.004-.01\end{array}$} & \multirow{2}{*}{$\begin{array}{l}\text { Angular and well sized. } \\
\text { Do. } \\
\text { Contains considerable proportion of } 0.06 \text {, also much } \\
\text { fine material, poorly sized. } \\
\text { Contains a considerable proportion of } 0.025 \text {, also much } \\
\text { fine material, poorly sized. }\end{array}$} \\
\hline 65 alundum........... & & & \\
\hline
\end{tabular}

These observations are more or less of a qualitative nature but serve to show the relative characters of the different abrasives. This is of some importance, for the trade names give no indication of real sizing of the material. These observations were first made by Sampson. They have been checked and supplemented by the present writer and further checked by C. S. Ross. 
Impregnating the specimen.-By far the greater proportion of specimens to be polished are strong enough to maintain themselves during the operations of grinding and polishing without noticeable disintegration. In ores that are porous and crumbly, however, the grains tend to work loose before they are completely polished, and when they are carried across the face of the specimen they groove and - scratch it. This may be avoided by filling the pore spaces with some binding medium that is carried into the pore spaces by capillarity or under a vacuum. The binding medium must be dissolved in some fluid of low viscosity, in order that the resulting solution can readily enter the pores. The section is heated sufficiently to evaporate the solvent, leaving the binding medium in the pores. As impregnation is most effective at the outer surface of the specimen it is advisable to give a flat surface to the specimen before it is impregnated. Some specimens are too crumbly to permit grinding even a rough flat surface. Such specimens should have a first impregnation before grinding and a second impregnation afterward.

The technique of impregnation with bakelite varnish has been described by Ross, ${ }^{12}$ who developed the method as used in making thin sections of friable rocks. In his paper in Economic Geology a method of impregnating specimens with bakelite under a vacuum is described.

The procedure described by Ross is highly successful with polished ores. The specimen is immersed in a dish containing the solution of bakelite in ether and alcohol or ether and acetone. The bakelite should be thinned to about the consistency of a thin watery syrup. The dish is covered to prevent too rapid evaporation of the solvent. The bakelite solution is drawn into the pores of the specimen by capillarity. After four to eight hours the cover is taken off the dish, and the contents are exposed to the air over night. The solution is then about the consistency of unthinned bakelite or possibly still thicker. The specimen is then removed from the dish and placed with its flat side uppermost in a warming oven. Care should be taken to have a generous coating of the solution on the surface and to keep the surface horizontal so that the solution will not run off. A temperature of approximately $40^{\circ} \mathrm{C}$. is maintained for about 24 hours. The temperature is then gradually raised to about $110^{\circ}$ C. and maintained there an additional 24 hours. At the end of that time the bakelite is hard and resembles amber. It is futile to attempt to hasten the process of curing bakelite. The application of too much heat or a too rapid increase in the heat will cause its decomposition and the formation of bubbles, which

\footnotetext{
is Ross, C. S., A method of preparing thin sections of friable rocks: Am. Jour. Sci., 5th ser.; vol. 7, p. 483, 1924 ; Methods of preparation of sedimentary materials for study: Econ. Geology, vol. 21, pp. 454-468, 1926.
} 
tend to blow out material already in the pores. The excess bake. lite is ground off the surface on a horizontal lap, with $\mathrm{F}$ carborun: dum. The specimen is then examined under the microscope. If impregnation has not been sufficient the entire process can be re peated, as cured bakelite is not affected by the solvent used. The second impregnation is usually not necessary, however, as bakelite holds the mineral grains firmly, and a specimen with 50 per cent of its pore spaces filled with bakelite will take a satisfactory polish.

The method above described is, in the writer's opinion, the best method yet devised for impregnating a specimen. It has the dis advantage, however, that it requires at least two and a half days for its completion. A method of impregnation in a vacuum with canada balsam and xylol that has been described by the writer ${ }^{15}$ nvolves much more work than the bakelite method, and the result is somewhat less satisfactory, but it can be accomplished in half an hour.

Another method that is quick and sufficiently satisfactory for most purposes involves the use of "kollolith," an artificial product somewhat similar to canada balsam; it is manufactured by Voigt \& Hochgesang, Gottingen, Germany. It is prepared in two grades, hard and soft. The hard grade is recommended for impregnating the specimen. A piece of kollolith is cut or broken off from the tube in which it comes, placed in a porcelain dish, and heated until it melts. The specimen is ground with a rough flat surface and then immersed in the melt, which is boiled for three minutes. The speci. men is then removed, care being taken to keep the flat surface upper. most so as to prevent the syrupy. melted kollolith from running off. On cooling the kollolith again hardens.

\section{POLISHING PROCESS AT HARVARD UNIVERSITY}

The most effective method of polishing yet described is the Van. derwilt method, ${ }^{14}$ devised in the Laboratory of Economic Geology, Harvard University. This method is based on two fundamental factors :

1. Polishing is a grinding process in which the grinding scratches are of submicroscopic width. To obtain a polish by fine grinding, the abrasives must be very carefully sized, and in the final grinding, the diameter of the largest grains must not exceed 0.001 millimeter. Vanderwilt devised a method of sizing grains based on slow circula. tion of the sludge through a series of cylinders. This method is slow but gives a much more uniform product than the quick method described on page 13 of this paper.

\footnotetext{
${ }^{13}$ Short, M. N., The preparation of polished sections of ores: Econ. Geology, vol. 21, p. 653,1926 .

16 Vanderwilt, J. W., Improvements in the polishing of ores: Econ. Geology, vol. 23, p. 292.1928.
} 
2. Cloth laps are dispensed with, and the polishing is done on laps made of lead. Water is excluded in the final stages of the process, and the abrasive is carried in oil. The laps run at a slow speed, the process from beginning to end requiring from 6 to 12 hours. The grinding and polishing are all done by machinery; there are four machines, each holding six specimens, and all stages of the process are carried on at the same time. With allowance for stoppage the time per specimen is from 20 to 40 minutes.

This method gives a surface of almost no relief, even between minerals of great contrast in hardness, such as pyrite and galena. It is superior to the usual polishing methods for preparing surfaces for microscopic observations and photonicrography at high magnifications. It is particularly valuable where it is desired to study thin alteration zones between hard and soft minerals, such as zones of incipient chalcocite enrichment along boundaries between pyrite and chalcopyrite. The principal disadvantages of the method are that it requires more time and a higher degree of technical skill than the usual methods, and an expensive apparatus is necessary.

\section{POLISHING PROCESS AT THE UNIVERISIT OF MINNESOTA}

In the process used at the University of Minnesota the first surface is ground on a horizontal wheel using a paste of 46 or 120 alundum. The flat surface thus obtained is beveled on a carborundum wheel. This is followed by hand grinding on a glass plate with alundum $5 \mathrm{~F}$, followed on another plate with alundum $60 \mathrm{~F}$. About one minute usually suffices for this step, but for some specimens more time can be spent to advantage. Polishing is accomplished on three vertical wheels driven at 1,800 revolutions a minute. The wheels are mounted with heavy linen or sheeting. The best grade of sheeting costs much less than linen and gives very satisfactory results. All abrasives are applied to these wheels with paint brushes dipped in the abrasive suspended in water. Alundum $60 \mathrm{~F}$ is used on the first wheel, chromic oxide (10 minutes) on the second, and either rouge (60 minutes) or magnesia for the final polish. Schwartz, ${ }^{15}$ who describes this process, states that the average amount of time spent for specimens presenting no unusual difficulties is from 6 to 10 minutes.

\section{PHOTOMICROGRAPHY OF POLISHED SECTIONS}

Photomicrographic camera.-Davy and Farnham ${ }^{16}$ state that For the mining geologist, photographs of the ore minerals and the relations between them, as seen by vertical illumination, are almost indispensable in pre-

\footnotetext{
${ }^{25}$ Schwartz, G. M., Discussion on polishing methods: Econ. Geology, vol. 22, p. 193. 1927.

${ }^{10}$ Davy, W. M., and Farnham, C. M., op. cit., p. 12.
} 
paring reports. A well-chosen illustration will establish a point more conclusively in the minds of the readers than pages of written description. For the student of ore deposits, such photomicrographs are invaluable records to be preserved as reference material.

Photomicrography is a very simple operation, far more so than is generally believed. All that is necessary is a camera box attached to the microscope, the objective and ocular of the microscope act. ing as a compound lens for the camera. The microscope itself

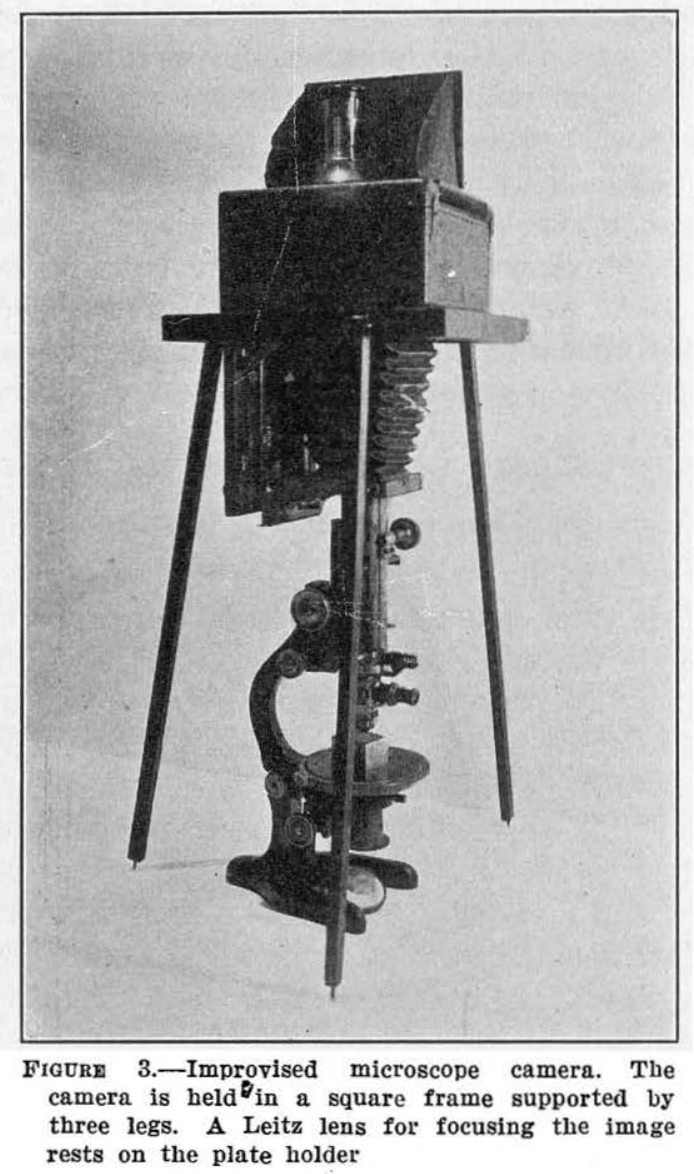

is a projection instrument and will project the image of the object on which it is focused. If a sheet of white paper is held horizontally above the ocular of the microscope, a circular image of this object is thrown on the paper. The diameter of the image depends upon the power of the ocular and the distance of the paper above it. An ocular of low magnification, such as the No. 1 Leitz, projects a conit of rays of relatively small vertical angle. An ocular of higher magnification projects a cone of rays with a wider angle, and con- 
sequently at a given distance the image projected is larger. The diameter of the image varies directly with the distance from the ocular, because the image is the base of a right circular cone of rays.

All that is necessary in photographing an image, then, is to place a photographic plate at a certain distance above the ocular of the microscope, the distance depending upon the size of the image desired, and to screen out all light other than that projected by the ocular of the microscope itself. The most essential requirement of the camera is that it be made rigid, to prevent vibration of the plate during exposure.

Figure 3 shows such an arrangement which gives excellent results. The camera is a 4 by 5 inch Folmer \& Schwing plate camera from which the lens has been removed; This is supported on a tripod, the legs of which are screwed onto the camera box. The plate holder is 57 centimeters vertically above the base of the tripod and 20 centimeters above the ocular of the microscope, with a 16-millimeter objective focused on a polished section mounted in brass. The tube length of the microscope with the vertical illuminator attached is 205 millimeters. The adjustable bellows provides for variations in focal distance. A black cloth provides a light-tight joint where the ocular enters the bellows. Differences in magnification are provided for by changing the objective or ocular. The No. 3 Leitz ocular just covers a 4 by 5 inch plate in the arrangement shown. With this ocular the different objectives give the following magnification: 32-millimeter Bausch \& Lomb, 29; No. 2 Leitz (24-millimeter), 49; 16-millimeter Bausch \& Lomb, 78; No. 4 Leitz'(9-millimeter), 144; 4-millimeter Bausch \& Lomb, 360.

This attachment does not provide a shutter for timing the exposure, which is accomplished by switching the electric light on and off. The exposure usually ranges from one second to ten minutes, hence a shutter that will give exposures of fractions of a second is not required.

The image is first focused as well as possible on a ground glass at the top of the camera; the ground glass is in the position that the photographic plate will later occupy and gives an accurate view of the image as it will appear in the photograph. It also enables the operator to equalize the illumination by turning the vertical illuminator or shifting the lamp. The light should be as strong as possible, and no filter should be used at first. If the minerals do not all show clearly, the effect produced by different color filters is then tried out by testing them in succession, as described on pages 24-25.

For an accurate focus the ground glass is inadequate, as the image produced is not sufficiently sharp, and a transparent plate 
of glass is required. This is conveniently obtained by soaking 8 photographic plate in hot water and scraping off the emulsion. Th ground glass is removed from the camera and the transparent glass put in its place. When this is viewed with the unaided eye no image is seen at first, and all that is visible is a strong spot of light in the ocular of the microscope. For this reason it is not generally known that it is possible to focus on a transparent glass. However, if a small hand lens is focused on the glass and the eye placed in a certain position, to be ascertained by trial, a sharp image appears. The optical axis of the hand lens must be parallel to the ray of light at that point, hence it is best to focus at the center of the plate, where the plate and medial plane of the lens are parallel. Some skill is necessary in detecting the image at first, but once successful the operator encounters no further trouble. With a little practice he almost instinctively locates the definite place where the image is seen. The image seen is only that part of the plate covered by the lens, but this image is sharp, and the amount of light required to give an observable image is only a small pro. portion of the light necessary to give a good image on the ground glass.

When the focus is obtained the transparent glass focusing plate is removed, and the plate holder with its inclosed photographic plate is inserted in its place. The slide of the plate holder is pulled out and the light turned on for the required exposure. The light is then turned off, the slide of the plate holder pushed back into place, and the plate holder removed and taken into a dark room, where the negative is developed and fixed.

The Leitz "Makam" camera attachment, which has been used in the Geological Survey laboratory, is similar to the improvised camera above described but has some features of advantage. It consists of 8 camera box with a $5 \mathrm{x}$ ocular. (The cost of this attachment is approximately \$86.) The ocular of the camera fits into the barrel of the microscope and takes the place of the usual ocular of the microscope. This supports the camera and thus obviates the need of a tripod sup. port. The camera has a horizontal eyepiece in a tube at right angles to the ocular. A prism placed in the optical axis of the camera just above the ocular throws the microscopic image through the eyepiece. The eyepiece can be moved in and out of its tube for the purpose of focusing. A lug attached to the eyepiece moves in a spiral slot, and by means of a circular vertical scale its position with the image in focus can be accurately located. The advantage of this arrangement is that it is not necessary to focus the image on the ground glass of transparent glass every time a picture is taken. The eyepiece is adjusted so that the image is in focus at the same time that it is in focus on the ground glass. Then when additional pictures are taken 
it is only necessary to focus the image in the eyepiece. However, the adjustment of the eyepiece varies for each objective, but it can be accurately recorded by means of the circular scale. Immediately before the photographic plate is exposed the prism that throws the image through the eyepiece is removed from the path of the light rays by pressing on a spring release. This arrangement permits an almost

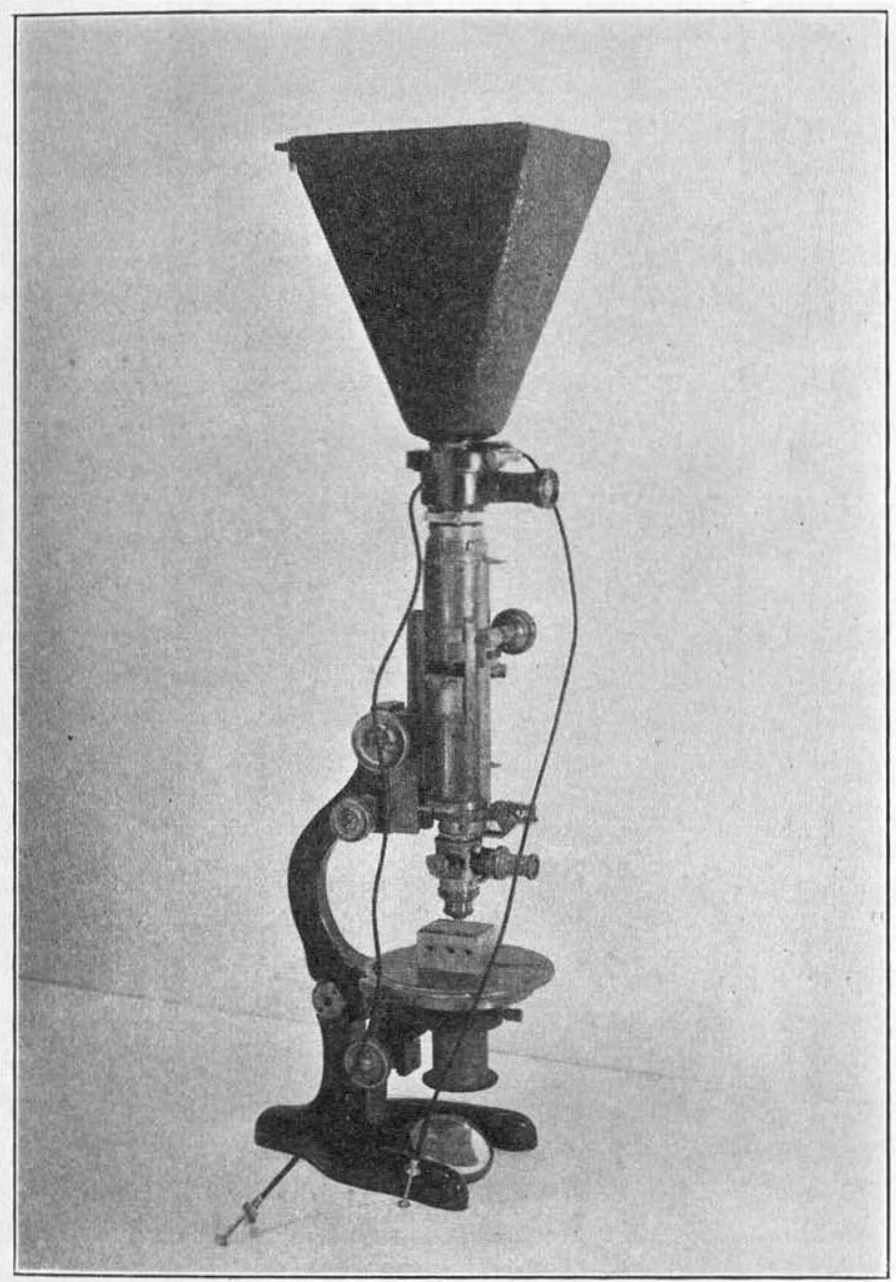

Figure 4.-Leitz " Makam" camera attachment

instantaneous focus before exposure and is of particular advantage in photographing growing crystals. All the photographs of microchemical tests shown in part 4 of this bulletin were taken with this attachment. The size of the photographs is $31 / 4$ by $4 \frac{1}{4}$ inches. As the camera attachment is an inverted pyramid, larger sizes would tend to make it more unstable and more readily affected by vibration. 


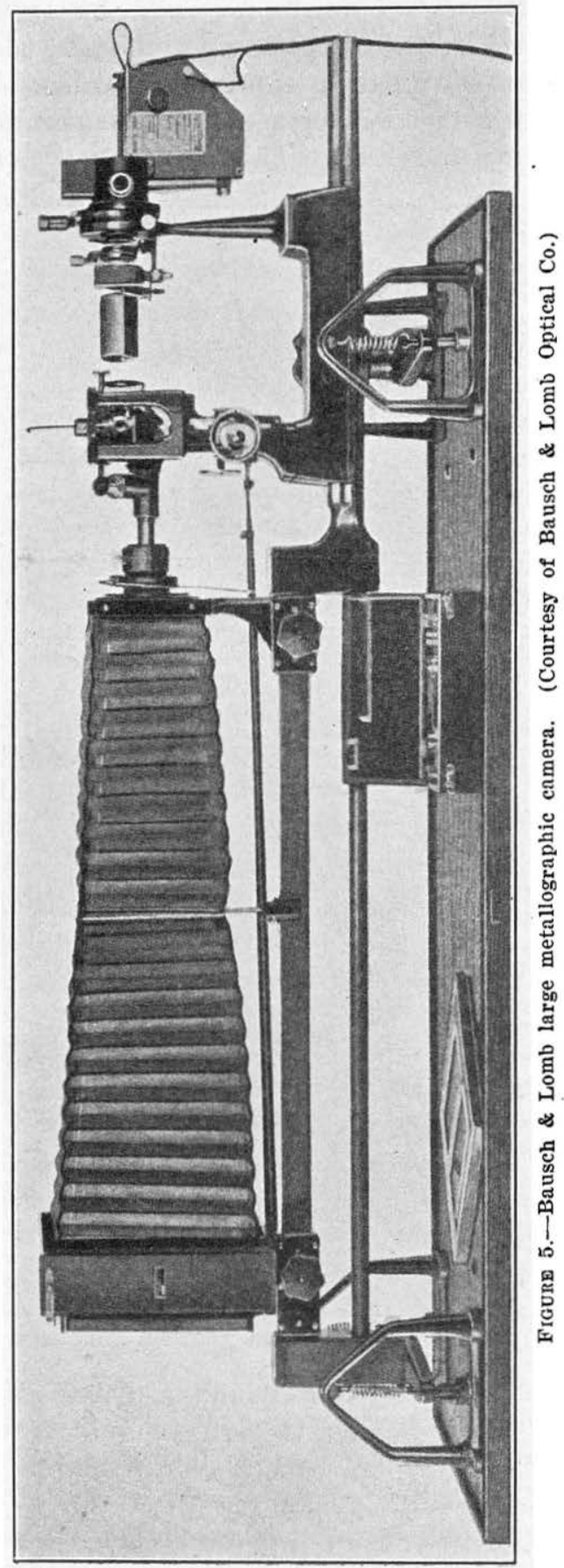


Satisfactory pictures with exposures as long as 12 minutes have been taken in the writer's laboratory with this attachment, though the laboratory is by no means free from vibration caused by heavy machinery. With longer exposures some precaution should be taken to protect the camera and microscope from vibrations, by waiting until the machinery has shut down and near-by street traffic is not heavy.

As the distance between the ocular and the plate holder is fixed, each objective gives a fixed definite magnification as follows: 32millimeter, 40; 24-millimeter, 68; 16-millimeter, 95; 9-millimeter, $170 ; 4$-millimeter, 385. The tube length of the microscope with vertical illuminator attached is 205 millimeters.

Many metallurgical laboratories and universities where much photomicrography of opaque specimens is carried on use the Le Chatelier model inverted microscope with camera attachment. This is manufactured by several firms, such as Leitz, Bausch \& Lomb, and Zeiss. The present writer uses the Bausch \& Lomb instrument shown in Figure 5.

The polished surface is placed face down on the stage of the microscope; hence no mounting is necessary, and the object can be of any size that the stage can support without undue strain. The bellows is horizontal and has an extensible length of more than 3 feet. The camera box, bellows, microscope, and lamp are all supported on a firm horizontal standard, which in turn is suspended from springs. The standard vibrates freely from the springs, and the camera and other fixtures vibrate as a unit with it, but during these vibrations the camera and microscope maintain the same relative positions, consequently there is no blurring of the picture. Vibrations in the building are transmitted in part to the springs of the instrument, but they have little or no effect on the picture. This instrument is better than the camera attachments already described for magnifications greater than 800 diameters. It is more convenient to operate, and, as the bellows has a wide range in length, magnifications of any desired magnitude can be taken. The instrument costs $\$ 482.50$ (January, 1930), however, and it is not very often that magnifications as high as 800 diameters will be required. Moreover, if the polished section has much relief, a magnification as high as this is not possible, as part of the picture will be out of focus. (See pl. 1, D.)

Light filters.-Photomicrographs of thin sections usually show sufficient contrast between the component minerals if photographed in white light, and no color filter is required. In fact, in many thin sections the minerals have too much contrast in light or color to photograph well without screening out some of the component 
colors of white light. The resulting negatives may appear satis. factory, but the printing paper does not permit as much contrast as the negative. A light-colored mineral is dense in the photo. graphic negative, whereas a dark-colored mineral is more trans. parent. If the contrast between the minerals in the negative is too great, the light-colored mineral will have to be printed so long in order to bring out the detail that the dark-colored mineral is overprinted and its detail obliterated. In this event some means should be taken to reduce the contrast between the minerals. This is accomplished by the use of red or yellow light instead of white. Inspection of many photomicrographs of thin sections in geologic papers indicates that this factor of overcontrast has not been suff. ciently taken into consideration.

On the other hand, the greater part of the minerals in a polished section are of various shades of gray, and the contrast between the colors may be so slight that boundaries between the component minerals are almost invisible. Under such conditions means must be taken to increase the contrast between the component minerals. This is accomplished by screening out some of the colors of white light. 'To get good results a panchromatic plate - that is, one which is sensitive to all colors of the visible spectrum-is required. The Wratten M plate, made by the Eastman Kodak Co., has been used by the writer and fills this requirement admirably. In the following descriptions this plate is specified.

A color filter is a sheet of colored emulsion between glass plates. When light passes through a green filter, for instance, the colors of the red end of the spectrum are absorbed, but the green, with some of the other colors near it in the spectrum, passes through. To get contrast between two minerals that are close together in the color spectrum, a filter that transmits light at the other end of the spectrum is chosen.

Let us take a specific example. Chalcopyrite is light yellow, and galena silvery white. It is desired to increase the contrast in color between them. If a yellow filter (the $K_{3}$ Wratten, for instance) is used, the chalcopyrite reflects yellow light, and its color is not materially changed by light passed through the filter. Galena, being white, reflects a large part of the visible spectrum, including yellow; but only the yellow rays and those near it in wave length reach the eye, as many of the rays at the other end of the spectrum were absorbed by the filter. Hence both chalcopyrite and galena reflect yellow light, and the contrast between them is lessened. It is obvious that a yellow filter is not suitable for bringing out a contrast between two yellow minerals or between a yellow and a white mineral. On the other hand, when a blue filter is used for chalcopyrite and galena, 


\section{PLATE 1}

\section{Photomicrographs of Polished Surface of Specimen from Magma Mine, Superior, Ariz.}

Pyrite (py), chalcopyrite (cp), bornite (bn), and quartz (qtz). Same field taken with different objectives to illustrate effect of relief. White rectangles on $A$, $B$, and $C$ show areas covered by $B, C$, and $D$, respectively. B (green) filter, Bausch \& Lomb inverted photomicrographic camera.

A. 32-millimeter objective, 3-minute exposure, $\times 47$. The black borders at the peripheries of pyrite grains are very pronounced. They are due to reflection from the beveled sides of the pyrite. (See p. 37.)

$B$. 16-millimeter objective, 2-minute exposure, $\times 112$. The black borders are much less pronounced than in $A$, owing to the fact that the cone of rays striking the surface is flatter than with the 32-millmeter objective, and more oblique rays strike the beveled edge of the pyrite. The 16-millimeter objective is the most satisfactory for most purposes.

C. 9-millimeter (No. 4 Leitz) objective, 1.5-minute exposure, $\times 200$. This picture is likewise satisfactory, but the objective was focused on the softer minerals, and the imperfections (small pits) on the surface of the pyrite are slightly out of focus. The black borders are very inconspicuous.

D. 4-millimeter objective, 2-minute exposure, $\times 455$. Pyrite is decidedly out of focus.

$15122-31-3$ 
U. S. GEOLOGICAL SURVEY
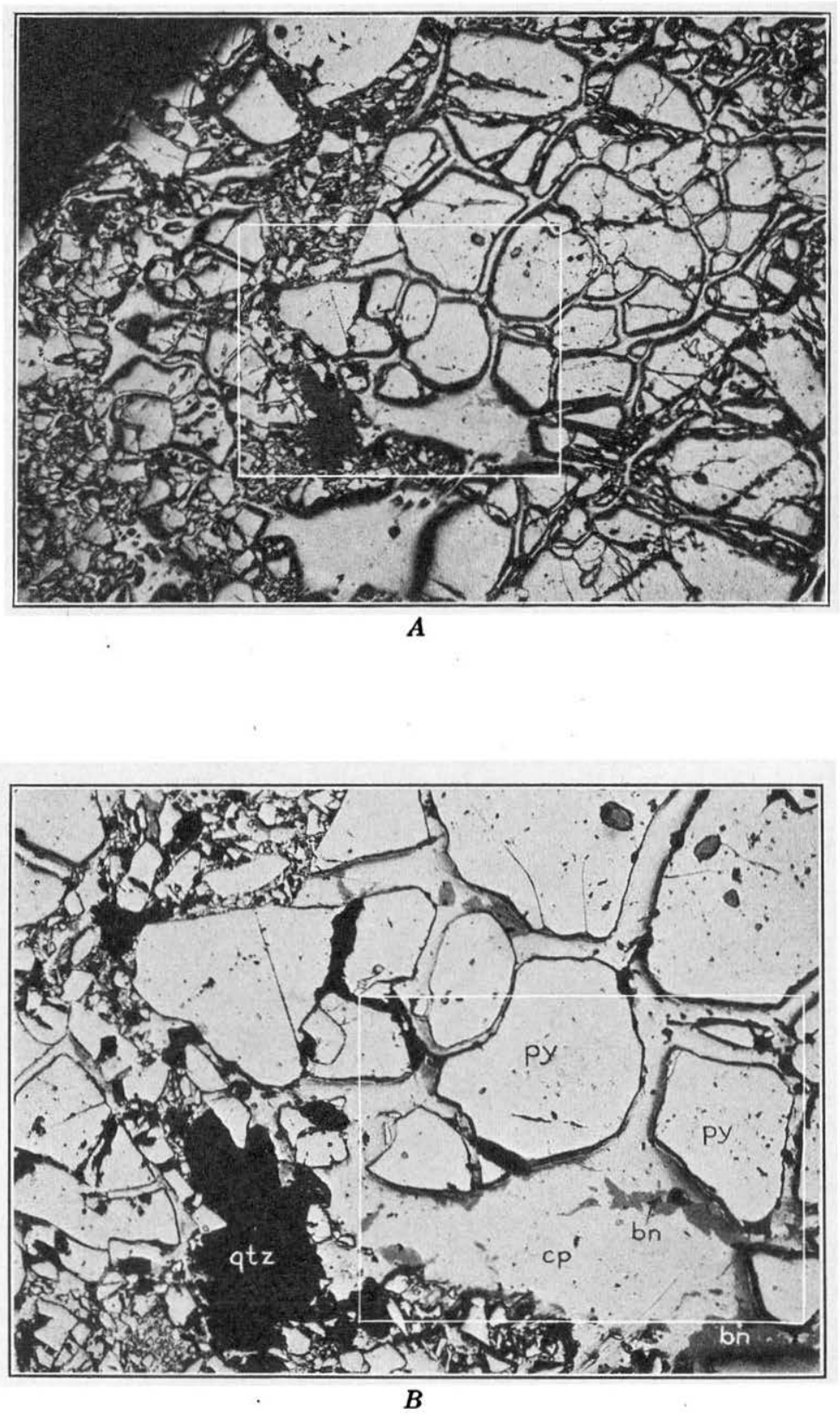
BULLETIN 825 PLATE 1
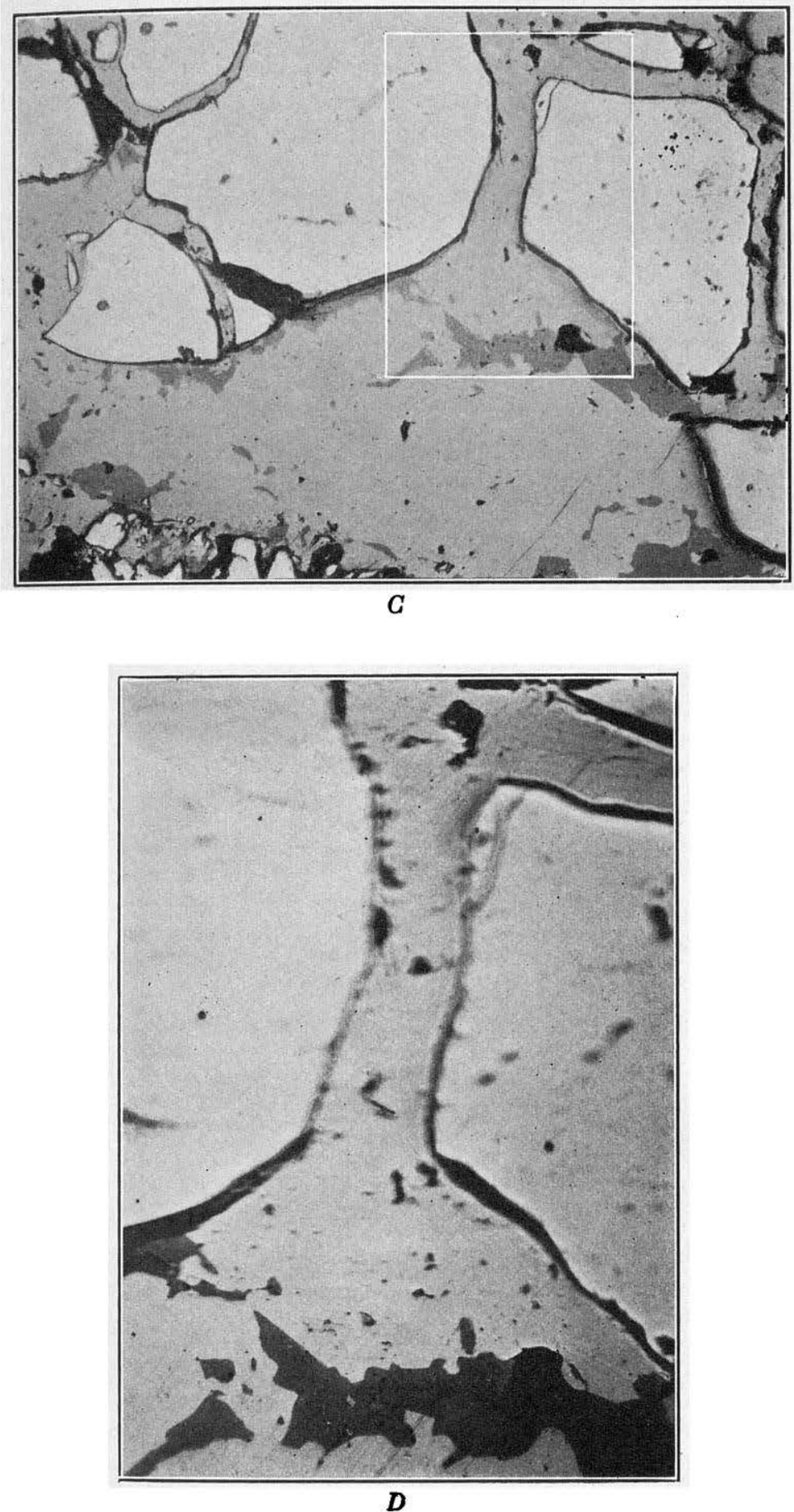

the galena, which reflects most of the colors of the spectrum, will reflect the blue components. The chalcopyrite absorbs colors near the blue end of the spectrum, and, as only blue rays reach it, the mineral will appear dark. Hence in the photograph the chalcopyrite will be darker than the galena.

The Wratten filters, made by the Eastman Kodak Co., have been tound highly satisfactory for this purpose. They are not completely monochromatic; indeed, this would be a disadvantage rather than an advantage. For instance, the blue filter transmits all the colors of the spectrum but not in the same proportion; it absorbs most of the red, transmitting only a small proportion of it. On the other hand, it transmits most of the blue but absorbs a small proportion.

The filters used by the writer are $\mathrm{A}$, red; $\mathrm{B}$, green; $\mathbf{K}_{3}$, yellow; $\mathrm{G}$, deep yellow; and $\mathrm{H}$, blue. Other colors have been recommended by different investigators, but the writer believes that the importance of having a particular shade of any color has been overestimated. A partial list of filters of this group that are suitable for different mineral combinations is as follows:

Chalcopyrite and galena, $\mathrm{H}$ is best; $\mathrm{B}$ very good.

Chalcopyrite and pyrite, $\mathrm{H}$.

Chalcopyrite and chalcocite, $\mathbf{B}$.

Chalcocite and bornite, any filter will do; no filter required.

Chalcocite, bornite, and chalcopyrite, B.

Mottled chalcocite, $\mathbf{B}$ needed.

Chalcocite and covellite, $\mathrm{H}$ (covellite prints dark, and means should be taken to reduce the contrast).

Specularite and magnetite, $\mathbf{H}$.

Bornite and covellite, $\mathrm{H}$ is best ; $\mathrm{B}$ gives very little contrast.

Bornite and tennantite, $\mathbf{B}$.

Argentite and galena, $B$.

Sphalerite, $\mathbf{K}_{3}$; with other filters this mineral appears too dark.

Gangue minerals, $\mathrm{K}_{3}$ or $\mathrm{G}$ is best; with other filters transparent minerals print too dark.

The $\mathrm{A}$ and $\mathrm{G}$ filters are good for reducing contrast.

The exposure factor of each filter with a particular kind of light is given by the manufacturer. This is the multiple of time required by the filter to give the same density of negative as the same picture taken with white light. - For instance, the photomicrograph of pyrite and chalcopyrite shown in Plate $1, B$, was taken with the Bausch \& Lomb inverted photomicrographic camera using a 16-millimeter objective and a tungsten incandescent lamp. The magnification is 112 diameters. This required an exposure of two minutes with a B filter and only 20 seconds with no filter. The negatives obtained were of the same density. The exposure factor of filter B is therefore 6 .

The following factors were obtained experimentally with an $M$ plate and a tungsten incandescent lamp and differ somewhat from 
those given by the manufacturer: No filter, $1 ; A, 2 \frac{1}{2} ; \mathbf{B}, 6 ; \mathbf{K}_{3}, 2 \frac{1}{2}$. $\mathrm{G}, \mathbf{5} ; \mathrm{H}, 21$.

The exposure factors vary with the thickness of the emulsion as well as with the color itself and the character of the light. I is best for the investigator to determine these items for himsell experimentally. This is best done by dividing the negative int: five strips and photographing each strip separately. The slide of the plate holder is pulled out until it covers only one-fifth of the width of the negative. With a colored pencil a mark across th: opposite side of the slide is made where the slide first meets the slot through which it passes. The slide is then pushed until it covers two-fifths of the width of the negative, and another pencl mark is made on the opposite side of the slide. The same procedurs is repeated for the other strips.

The exposures are made in geometrical rather than arithmetical ratio. For instance, the first strip is exposed for 10 seconds, the second 20 seconds, the third 40 seconds, the fourth 80 seconds, and the fifth 160 seconds. If the last strip still looks underexposed when developed, the same procedure is followed with exposures of whole minutes-for instance, 1, 2, 4, 8, and 16 minutes. The manner in which the strips are exposed is as follows:

The slide is pulled out until the entire plate is uncovered, and the plate is exposed 10 seconds. Then the slide is pushed one-fift of the way across the plate, which is exposed another 10 seconds Qne-fifth of the plate has been exposed 10 seconds and four-fifths o: it a total of 20 seconds. Then the slide is pushed another fifth of thit distance across the plate and the rest of the plate exposed 20 seconds Three-fifths of the plate will have been exposed a total of 40 seconds Then the slide is pushed another fifth of the distance across the plat and the rest of the plate exposed another 40 seconds. The remaining two-fifths of the plate will have been exposed a total of 80 seconds The slide is then pushed until only one-fifth of the negative is un. covered and it is exposed an aditional 80 seconds, making a total for this strip of 160 seconds. The plate is developed and examined, and the strip showing the desired density is chosen; or if the desired density would be between those of two adjacent strips, the mean between the two exposure factors is selected.

If an arc lamp is used instead of a tungsten incandescent lamp the filter factors will be somewhat different from those given above and should be determined experimentally by the investigator. The an lamp may be necessary in taking pictures with very high magnifica. tion.

Exposure formula.-The time of exposure is affected by other factors, the most important of which are the numerical aperture of 
the lens used and the magnification. The aperture of a lens is defined as the angle of the widest cone of rays which the lens can utilize. The numerical aperture (usually marked on a lens by the letters $\mathrm{N}$. A.) is defined as the product of the sine of one-half the angle of aperture and the refractive index of the medium (air, oil, etc.) in which the objective works. The longer the focus and the lower the magnification of a lens, the smaller will be its numerical aperture. The exposure of the negative varies inversely as the square of the numerical aperture. The following table gives the numerical apertures of the different lenses used by the writer and the relative exposure factors calculated in comparison with that for the 16-millimeter lens.

\begin{tabular}{|c|c|c|c|}
\hline Lens & N. A. & $\left(\frac{1}{\text { N.A. A. }}\right)^{2}$ & $\begin{array}{c}\text { Approximate } \\
\text { factor }\end{array}$ \\
\hline $\begin{array}{l}\text { 32-millimeter. } \\
\text { 24-millimeter ( } \mathrm{No} 2 \text { Leitz) } \\
\text { 16-millimeter... }\end{array}$ & $\begin{array}{l}0.10 \\
.20 \\
.25\end{array}$ & $\begin{array}{r}100 \\
25 \\
16\end{array}$ & $\begin{array}{l}6.2 \\
1.7 \\
1.0\end{array}$ \\
\hline 9-millimeter (No. 4 Leitz).. & .45 & 5 & $.31=\frac{1}{3.2}$ \\
\hline 4-milimeter... & .85 & 1.4 & $.086=\frac{1}{11.6}$ \\
\hline
\end{tabular}

The exposure of the negative varies directly with the square of the magnification. Hence the numerical aperture factor and the magnification factor tend to balance each other. To take a specific example: The negative of Plate $1, B$, was exposed 2 minutes with a $\mathrm{B}$ filter, a 16-millimeter lens, and a tungsten incandescent lamp with a magnification of 112 . The calculated exposure required for a 32-millimeter lens to take the same picture with a magnification of 47 is 2 minutes $\times 6.2 \times \frac{47^{2}}{112^{2}}=2.2$ minutes. The exposure actually used, which was determined by exposing successive strips of the negative, was 3 minutes. (See pl. 1, A.) It is apparent that the factor above given is not very accurate.

The same procedure was applied to the other lenses, and the total results may be tabulated as follows:

\begin{tabular}{|c|c|c|c|}
\hline \multirow{2}{*}{ Lens } & \multirow{2}{*}{$\begin{array}{l}\text { Magniflca- } \\
\text { tion }\end{array}$} & \multicolumn{2}{|c|}{ Exposure (minutes) } \\
\hline & & Calculated & $\begin{array}{c}\text { Determined } \\
\text { experimentally }\end{array}$ \\
\hline 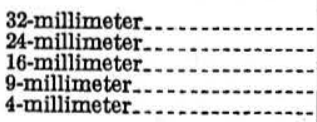 & $\begin{array}{r}47 \\
70 \\
112 \\
200 \\
455\end{array}$ & $\begin{array}{l}2.2 \\
1.33 \\
2.0 \\
2.0 \\
2.85\end{array}$ & $\begin{array}{l}\text { 3. } 0 \\
2.67 \\
2.0 \\
1.5 \\
2.0\end{array}$ \\
\hline
\end{tabular}


It is evident that the objectives of lower magnification are slower and the objectives of higher magnification faster than the calcula. tions would indicate. A careful record of exposures should be kept by the investigator, and this will prove much more useful in determining future exposures than the factors above given.

Developing and printing.-Panchromatic plates must be developed in total darkness or with the aid of the special Wratten green safe light. The writer prefers to work in total darkness, giving the development a specified time depending on the temperature. The plate is developed in a tray, then removed, rinsed in water, and placed in the fixing bath.

The pyro or elon-hydrochinon developers, formulas for which are given on a card inside the box containing the Wratten $\mathbf{M}$ plates, are satisfactory. The writer prefers, however, to use Rodinal developer, which is prepared by the Agfa-Ansco Corporation and sold in liquid form in 16-ounce bottles. Diluting 5 cubic centimeters of the solution by adding 95 cubic centimeters of water makes sufficient solution for a 4 by 5 inch tray. Rodinal developer oxidizes less rapidly than pyro developer and consequently may be used for several plates. If the developing is done within three hours, six or more plates may be developed in the same solution. As more plates are developed the solution begins to lose its strength, and it is better to discard it than to add to the time of development purely by guesswork. The author prefers to take and develop one picture at a time. This may seem to be less economical of time than taking several pictures at once and developing them together, but it is questionable if, in the long run, any time is gained by the latter procedure. By taking and developing the pictures one at a time errors in procedure or defects of apparatus or materials are revealed at once, and immediate steps can be taken to correct them.

The time of development depends on the temperature, as follows: $80^{\circ} \mathrm{F}$., 1.5 minutes; $75^{\circ}, 2$ minutes; $70^{\circ}, 3$ minutes; $65^{\circ}, 4$ minutes. It is best to use an acid fixing bath. Plates fixed in this bath do not require absolute darkness. Once they are placed in the fixing solution, a moderate amount of daylight can be admitted. A good formula is as follows: Sodium thiosulphate ("hypo"), 300 grams; potassium metabisulphite, 25 grams; water, 1,000 cubic centimeters. Fixing is continued until the milkiness has disappeared from the emulsion when viewed from the opposite side of the plate. The negative is then washed in running water for half an hour and dried.

The choice of printing paper depends on the contrast between the minerals as seen in the negative. If this contrast is weak, a contrasty paper is required; a contrasty negative requires a paper giving less contrast. 
Glossy paper is recommended, as it shows more detail than the different matte papers. The prints are mounted on a ferrotype plate. References.-The subject of photomicrography is too extensive and involved to be described here in all its remifieations. A very excellent textbook is Handbook of Photomicrography, by $\mathrm{H}$. Lloyd Hind and W. Brough Randles, published by George Routledge \& Sons (Ltd.), London, 1927. Another is the booklet Photomicrography published by the Eastman Kodak Co. 


\section{PART 2. PHYSICAL PROPERTIES}

\section{COLOR}

The color of a transparent mineral in reflected light depends largely on its index of refraction. Minerals of low index have low reflective capacity and absorb most of the incident light. The zeolites are nearly black, calcite and quartz are dark gray, sphalerite is light gray. In oblique incident light these minerals show internal reflection. This is favored by minute seams and cleavage cracks in the interior of the grain, which reflect the light. The internal reflection of a mineral usually gives the same color as the mineral itself in the hand specimen. Quartz is white, sphalerite brown, cuprite red, etc. The internal reflection is best seen by shutting off the light from the vertical illumi. nator and tilting the lamp so as to shine obliquely on the surface of the specimen.

Opaque minerals, if well polished, reflect nearly all the light im. pinging upon them and therefore, in vertically reflected light, appear lighter than the transparent minerals. Only a small proportion of the opaque minerals have distinct colors in vertically reflected light By far the greater number are white or have various shades and tints of gray. The minerals with distinctive colors are recognizable at sight or after scratching with a needle. With practice, color memory may be developed so highly that the investigator can recognize at a glance some of the more common white and gray minerals, as well as those with more distinctive colors. Murdoch ${ }^{17}$ says with reason, "The beginner in this work may at the outset experience some diff. culty in this matter of color determination, but it has been found that the ability to distinguish minute differences in color grows very rap. idly with experience, and it is astonishing how much more can be seen after a week's work than at the start."

Color is perhaps the most valuable property of a mineral in aid. ing in its identification. By means of color, crystal habit, and relief, all the more common ore minerals, such as pyrite, pyrrhotite, chalcopyrite, sphalerite, arsenopyrite, galena, and hematite, can be recognized at sight. This is fortunate, for if it were necessary to go through the etching and microchemical tests for each mineral, the amount of time required to examine a suite of specimens would be prohibitive.

${ }^{17}$ Murdoch, J., op. cit., p. 28. 
It is difficult to describe the color of a polished surface of a mineral in such a way that one not familiar with it can recognize it by its color. Talmage, ${ }^{18}$ in a very able discussion of the value of color, says:

Descriptions of color are subject to nearly as many interpretations as there are interpreters, no one of whom may get near to the idea that the describer intended to convey. Likewise, descriptions of the same color by different investigators show variation. Take, for example, a very common mineralpyrrhotite. Its color is variously described as light bronze, cream, pale reddish yellow, pale creamy brown, bronze-yellow, and pale brownish cream, but no one can know just what it looks like from any or all of these descriptions. When once a man has worked with it, the term "pyrrhotite color" conveys to his mind a very definite idea.

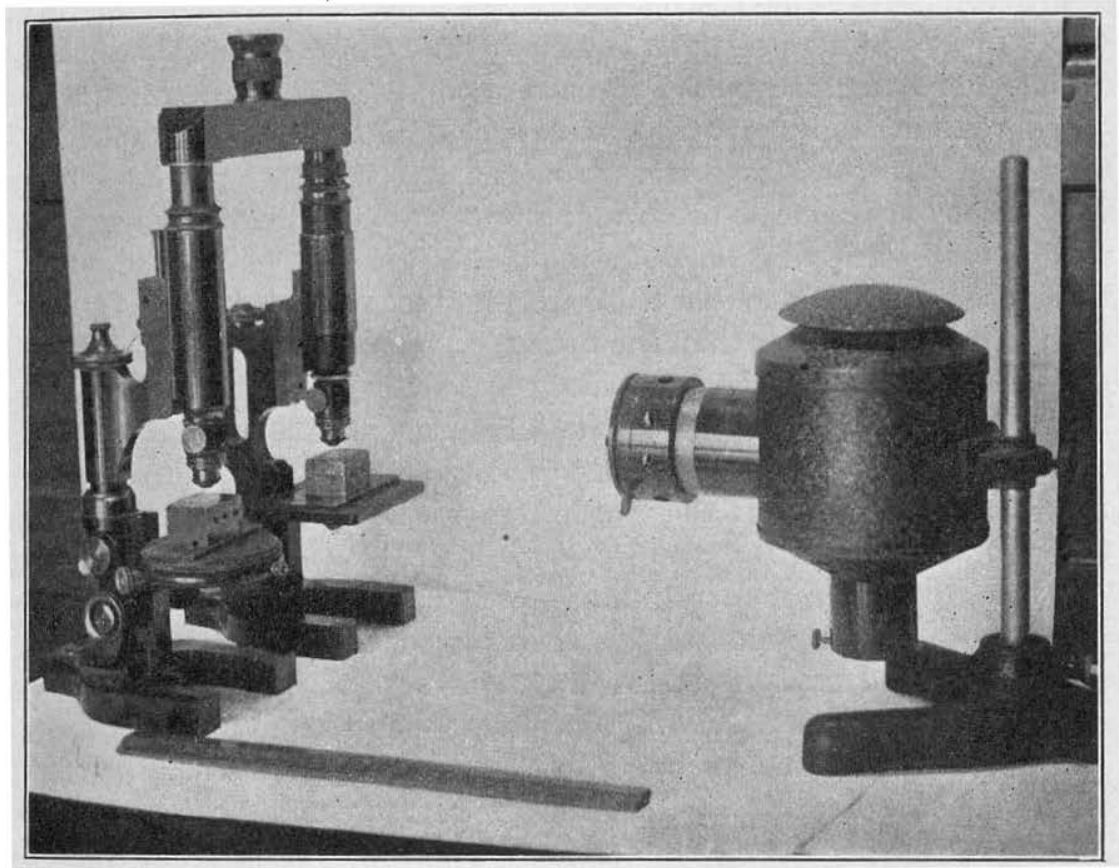

FIGURe 6.-Comparison microscope

With these statements the writer is in complete agreement. Pyrrhotite has a definite color, but it is elusive and defies accurate description. Once this color has been fixed in mind by examination of a sufficient number of polished surfaces of pyrrhotite, it can be recognized immediately. Cubanite $\left(\mathrm{CuFe}_{2} \mathrm{~S}_{3}\right)$ and perhaps one or two other minerals have the "pyrrhotite color."

The same thing is true of sphalerite, which has a peculiar gray color with a tinge of blue. With some practice this color can be recognized with unerring accuracy, but no description, however

\footnotetext{
${ }_{18}^{18}$ Talmage, S. B., Diagnostic value of color in polished sections: Econ. Geology, vol. 20, p. 171,1925 .
} 
wordy, can convey to the mind an accurate mental picture of the "sphalerite color." Davy and Farnham are particularly fond of the description "cream-color" and have applied it to pyrite, marcasite, pyrrhotite, chalmersite, pentlandite, and several other minerals. In the writer's opinion, none of these minerals resembles cream in color. Pyrite, marcasite, pentlandite, and millerite closely resemble one another in color and could be described as pale yellow.

It should not be forgotten that the ability to distinguish colors varies with different people; and this renders it difficult to apply any systematic classification on the basis of color, such as that set up in Murdoch's book.

The comparison microscope is the best instrument for comparing colors of two minerals not in contact with each other. This consists of two fixed microscope tubes, each with its own objective and reflecting illuminator. Instead of two oculars there is only one, between the tubes, and the images from the objectives are projected into it by means of prisms. The ocular shows both surfaces under examination, each occupying half of the field. Carefully paired objectives and reflectors are necessary. Talmage recommends the glass-disk type of reflector rather than the prism, as it gives a more equal illumination. Uniformity of illumination is a necessity and is insured by obtaining polished sections of the same specimen of galena, placing one on each stage, and adjusting the light source and the reflectors until the appearance of the two halves of the single field, as viewed through the comparison eyepiece, is identical. ${ }^{19}$

Work with the comparison microscope convinced Talmage that no two minerals, even among those classed as white and gray, have the same color, and hence that anyone, regardless of his color sensitiveness, could observe a color difference with such a microscope.

The comparison microscope also brings out the fact that the color of a mineral depends in part on its environment. According to Talmage, ${ }^{20}$

Chalcopyrite, in a field of gray or white minerals, looks brass-yellow; against pure native gold chalcopyrite looks a dirty olive-green, and if adjacent to native copper, chalcopyrite looks nearly grass-green. Tennantite and tetrahedrite are another pair almost indistinguishable. But by comparing the two with galena, the tennantite looks faintly olive-green, while the tetrahedrite looks brown with the green suggestion.

Many attempts have been made to define actual color values for the ore minerals. These efforts have met with only partial success. Murdoch ${ }^{20 a}$ says :

10 Talmage, S. B., op. cit., p. 173.

${ }^{20}$ Idem, p. 174.

${ }^{203}$ Murdoch, J., Microscopical determination of the opaque minerals, p. 28, New York, 1916. 
Except in the case of the distinctly colored minerals, the differences in color are so slight that even spectroscopic measurements of the reflected wave length would probably be ineffective. The books on colors and color standards deal with far brighter colors than most of the tinted white minerals show. Ridgway's book on "Color standards and nomenclature," giving 1,100 named colors, Is the best of this sort. He gives colored plates reproducing the shades and tints of the spectrum colors, with a white blank for comparison with the lightest tint of each color. Unfortunately, except for the distinctly colored minerals, which are relatively few in number, all the sulphide minerals have colors falling somewhere between pure white and his lightest tint, usually much nearer the white end, so his standards are of little use in this connection.

Schneiderhöhn ${ }^{21}$ uses a drawing ocular or camera lucida, by means of which he reflects the image of the mineral on the white-paper margin of a color chart. This scheme seems to the writer to have less merit than direct comparison by means of a comparison microscope.

The writer suggests that the white and gray minerals be divided into four classes by comparison with native silver, the whitest, and sphalerite, the darkest, of the ore minerals as follows:

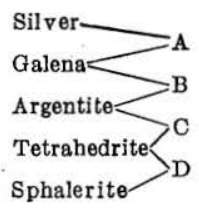

Chalcocite, for instance, is darker than galena and lighter than argentite and thus belongs in class $\mathrm{B}$. This scheme would require a comparison microscope, and for the present it is not used in the determinative tables.

For work with the comparison microscope daylight does not furnish a satisfactory illumination, as it varies too much during the day and is affected by cloudiness and other atmospheric changes. The artificial light Talmage recommends consists of a 75-watt 115volt blue ground-glass lamp, screened by Wratten filters Nos. 78b and 78c. "This arrangement," he says, "gives a light value well within the normal daylight range and also gives good visual color values, minerals under this illumination looking just about as they do in daylight."

The following minerals when viewed in vertically reflected light exhibit colors other than shades of white or gray:

\footnotetext{
${ }^{21}$ Schneiderhöhn, H., Mikroskopischen Bestimmung und Untersuchung von Erzen, p. 91, Berlin, 1922.
} 
Colored minerals

\begin{tabular}{|c|c|c|}
\hline Color & eral & Composition \\
\hline 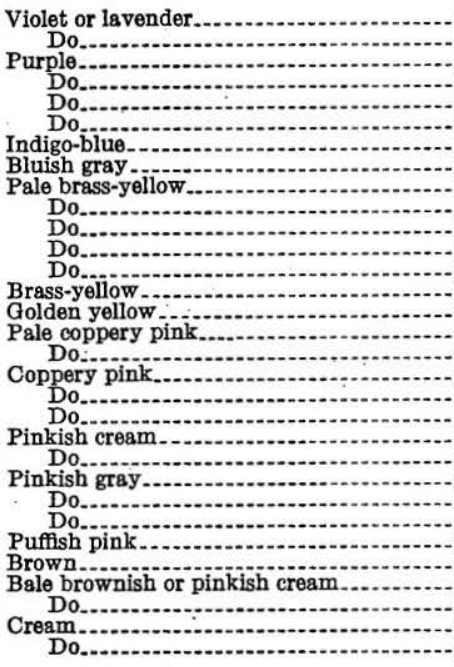 & 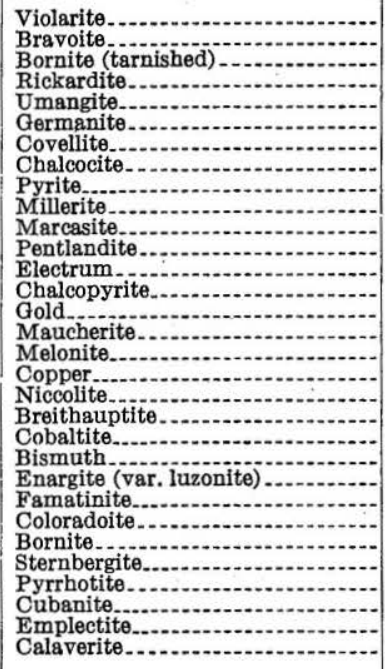 & 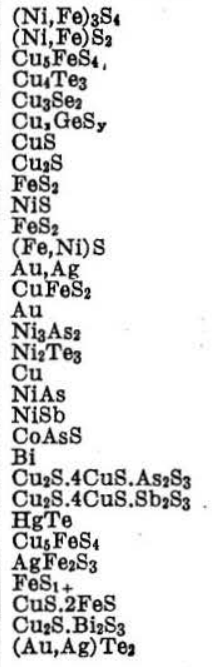 \\
\hline
\end{tabular}

Polished sections of many transparent minerals show distinctive colors when observed microscopically under oblique instead of vertically incident light. Seams, cracks, and cleavage planes below the surface of the section reflect back part of the light penetrating into the mineral. The color of a mineral under these conditions is usually nearly the same as that of the powder of the mineral. Internal reflections are also observed in vertically reflected polarized light (between crossed nicols) when the illumination is strong.

Minerals that show distinctive internal reflections

\begin{tabular}{|c|c|c|}
\hline Color of internal reflection & Mineral & Composition \\
\hline 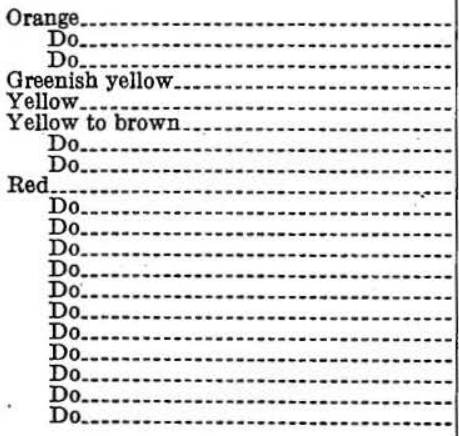 & 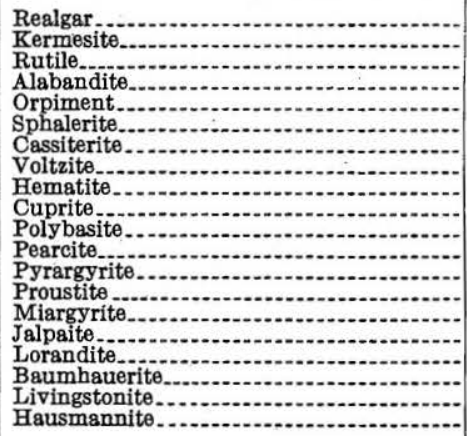 & 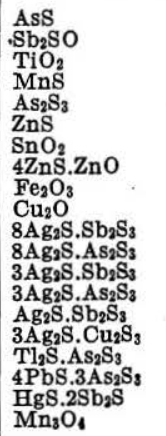 \\
\hline
\end{tabular}


Nonmetallic minerals and some metallic minerals, such as sphalerite, hübnerite, and cassiterite, give powders ranging from white to light brown in color. Transparent or translucent minerals distinctly colored in the hand specimen - that is, in ordinary oblique lightusually yield powders of nearly the same color. These minerals are usually gray or bluish gray in reflected light. The powders are best obtained under the reflecting microscope by holding the needle at an inclination to the polished surface and pushing it against the surface, at the same time giving the needle a rotary motion. The powder tends to collect as windrows on both sides of the groove.

Minerals that give characteristic colored powders

\begin{tabular}{|c|c|c|c|}
\hline Color of powder & Mineral & Composition & $\begin{array}{l}\text { Color of mineral in } \\
\text { vertically reflected } \\
\text { light }\end{array}$ \\
\hline 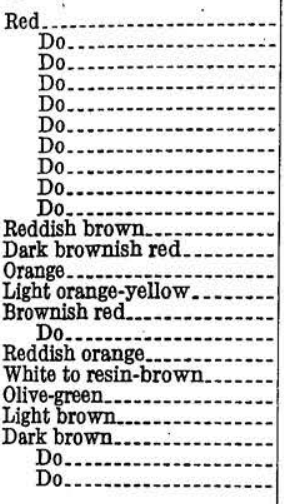 & 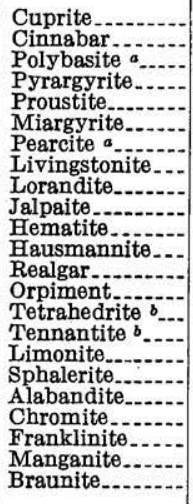 & 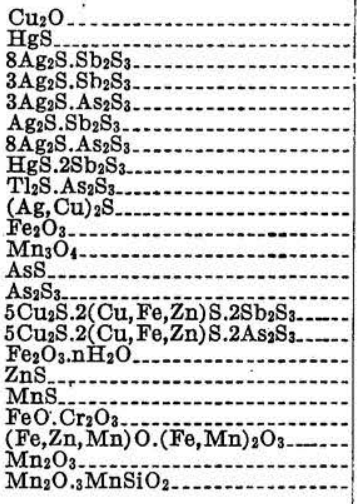 & $\begin{array}{l}\text { Bluish gray. } \\
\text { Do. } \\
\text { Gray. } \\
\text { Bluish gray. } \\
\text { Do. } \\
\text { Ashy gray. } \\
\text { Greenish gray. } \\
\text { Gray. } \\
\text { Do. } \\
\text { Do. } \\
\text { Bluish gray. } \\
\text { Do. } \\
\text { Do. } \\
\text { Gray. } \\
\text { Do. } \\
\text { Do. } \\
\text { Do. } \\
\text { Bluish gray. } \\
\text { Do. } \\
\text { Gray, like sphalerite. } \\
\text { Do. } \\
\text { Gray. } \\
\text { Do. }\end{array}$ \\
\hline
\end{tabular}

- Most specimens give black powder.

$b$ Some specimens give black powder.

\section{HARDNESS}

There is lack of agreement among various authorities as to the definition of hardness. ${ }^{22}$ Methods of measuring the relative hardness of materials may be briefly outlined as follows:

1. Scratching with a needle or other sharp instrument.

2. Pressure exerted on the surface by means of a steel ball.

3. Impact on the surface by dropping a steel ball on it.

4. Grinding the surface on a steel lap, with emery or similar abrasive, the length of time required to grind away a certain weight of a mineral of standard size and shape affording a measure of the hardness.

5. Boring with a diamond point of standard size and shape, the number of revolutions of the drill required to bore a definite depth in the mineral affording a measure of the hardness.

Of the methods cited, those employing scratching are the most applicable to minerals in general and are the only ones suitable for

\footnotetext{
${ }^{22}$ For an excellent discussion of hardness in minerals see Talmage, S. B., Quantitative standards for hardness of the ore minerals: Econ. Geology, vol. 20, pp. 531-553, 1925.
} 
use on polished surfaces under the microscope. The scratching method most used up to the present time is that described by Murdoch. ${ }^{23}$ A No. 10 Sharps needle is mounted in a handle 5 or 6 inches long, weighing a quarter of an ounce. The needle is brought into the field of view of the microscope and is held at an angle to the polished surface and dragged (not pushed) across the surface with its point inclined downward and to the rear. Murdoch recognized three grades of hardness, as follows:

1. Soft, or low hardness, including minerals that can be scratched with the weight of the handle alone or very easily scratched with light pressure.

2. Medium hardness, including minerals that can be scratched only faintly with slight pressure but easily with moderate or heavy pressure.

3. Hard, or high hardness, including minerals that can be scratched but slightly or not at all with heavy pressure.

The writer has used this method as well as he could for some years, but many serious errors have resulted from incorrect determinations based on it. A needle point will remain sharp for only a small number of hardness tests; it tends to curl like the toe of a ski, and thus its effectiveness for scratching is lessened.

The ore minerals exhibit all grades of hardness from the hardest to the softest, and any scheme of subdividing them is arbitrary; overlaps are inevitable. Moreover, some minerals vary considerably in hard. ness in different crystallographic directions.

There are more minerals which approximate the boundary between the medium and soft of Murdoch's three grades of hardness than between the medium and hard. A sharp needle will scratch chalcopyrite, tetrahedrite, and sphalerite, minerals listed by Murdoch and by Davy and Farnham as of medium hardness. The average worker is unable to decide whether these minerals scratch easily, thus falling in the "soft" group, or whether they scratch faintly, thus falling in the "medium" group.

For the reasons above set forth the writer has decided to dispense with the "medium" class of Murdoch and to place the minerals hitherto classed as of medium hardness in the "soft" class. In the scheme here adopted two grades of hardness are recognized:

1. Soft, including minerals which are scratched readily by a needle.

2. Hard, including minerals which are scratched with difficulty or not at all by a needle.

There are, no doubt, minerals as to which the investigator is puzzled to decide whether they scratch easily or with difficulty. This is inevitable, and experience is the only guide in aiding in a decision.

Observations of hardness as shown by resistance to wear are useful to a moderate extent. If two adjacent minerals are of different

${ }^{23}$ Murdoch, J., op. cit., p. 29. 
hardness the harder mineral will stand up in relief above the softer mineral. As polishing methods are improved this relief decreases. A scratch passing from a softer mineral to a hard is less distinct in the harder mineral than in the softer. In addition, an effect somewhat similar to the Becke ray used in petrography to detect differences of index of refraction between two adjacent transparent minerals is of value in determining differences of hardness between two adjacent opaque minerals. The harder mineral has a border that slopes downward toward the softer mineral. Light striking this border is reflected outside of the field of view. A dark band thus results. Adjoining this dark border on the side of the harder

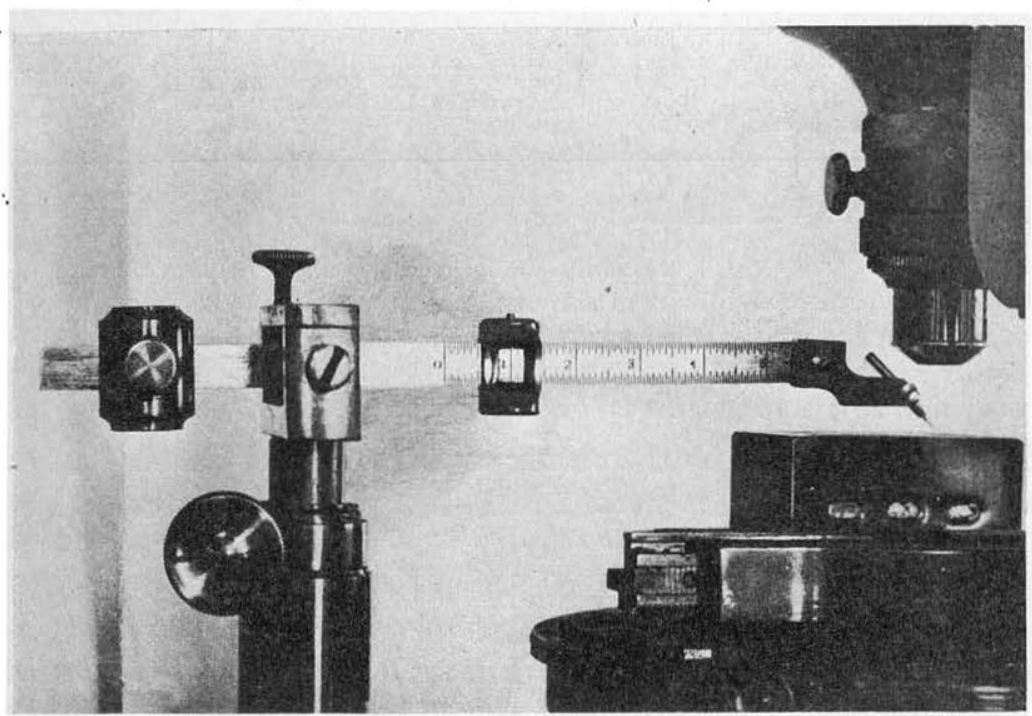

Figure 7.-Talmage hardness machine. Detailed view of scratching mechanism. (After Talmage. Courtesy of Economic Geology)

mineral and parallel to it is a line of light. When the tube of the microscope is raised, this bright line of light is seen to move from the hard to the soft mineral; when the tube is lowered the reverse takes place. ${ }^{24}$

An instrument for measuring the relative hardness of minerals in polished sections was recently described by Talmage. ${ }^{25}$ It consists essentially of a diamond attached to the end of a graduated beam. 'The beam carries a sliding weight like that of a weighing scale. The edge of the diamond is wedge-shaped, like that of an ax. The beam rides horizontally on jeweled pivots and balances when the adjustable weight is at zero. The apparatus is so arranged that the

${ }^{24}$ Van der Veen, R. W., op. cit., p. 28.

${ }^{25}$ Talmage, S. B., Quantitative standards for hardness of the ore minerals: Econ, Geology, vol. 20, pp. 535-543, 1925 . 
scratching point is directly under the objective of the microscope and the entire operation of scratching can be watched under the microscope. By means of the movable weight, Talmage determined the pressure necessary to produce a scratch of given character, called by him the standard scratch. The diamond remains stationary and the specimen is moved under it by means of a micrometer stage.

Talmage divides the minerals into seven classes of hardness, as follows :

\begin{tabular}{|c|c|c|c|c|c|c|c|}
\hline Designation & Type mineral & $\begin{array}{l}\text { Position } \\
\text { of weight } \\
\text { on beam }\end{array}$ & $\begin{array}{c}\text { Actual } \\
\text { weight on } \\
\text { scale } \\
\text { (grams) }\end{array}$ & Designation & Type mineral & $\begin{array}{l}\text { Position } \\
\text { of weight } \\
\text { on beam }\end{array}$ & $\begin{array}{c}\text { Actual } \\
\text { weight on } \\
\text { scale } \\
\text { (grams) }\end{array}$ \\
\hline $\begin{array}{l}\mathrm{A} \\
\mathrm{B} \\
\mathrm{C}\end{array}$ & $\begin{array}{l}\text { Argentite...... } \\
\text { Galena... } \\
\text { Chalcopyrite... } \\
\text { Tetrahedrite.. }\end{array}$ & $\begin{array}{r}0.2 \\
.4 \\
.7 \\
1.0\end{array}$ & $\begin{array}{r}0.105 \\
.210 \\
.365 \\
.520\end{array}$ & $\begin{array}{l}\mathrm{E} \\
\mathrm{F}\end{array}$ & $\begin{array}{l}\text { Niccolite } \\
\text { Magnetite.... } \\
\text { Ilmenite...... }\end{array}$ & $\begin{array}{l}1.5 \\
2.0 \\
3.0\end{array}$ & $\begin{array}{l}17 \mathrm{~s} \\
1.5 \\
1.5\end{array}$ \\
\hline
\end{tabular}

\section{According to Vanderwilt, ${ }^{26}$}

This device gives highly reliable results that can be readily checked, pro vided the surfaces have an essentially scratchless polish. It is believed to yill far more accurate values of scratch hardness than any device hitherto employed On badly scratched or pitted surfaces the scratch made by the diamond edge can not be clearly seen and the test is not reliable.

Experiments made by the writer on Talmage's instrument indicate that the time required to make a reliable hardness test averages about 15 minutes.

This instrument is not manufactured by any instrument maker at present, and its construction would involve some difficulty. It is not yet certain whether another instrument could be constructed that would duplicate Talmage's numerical results, but undoubtedly one similar to it could be made, and the figures corresponding to the type minerals in Talmage's table could be determined without difficulty.

Talmage's classes A, B, C, and D correspond to the "soft" group as used by the writer, and classes F and G to the "hard" group. Class $\mathrm{E}$ is about on the boundary between hard and soft, and minerals with that hardness are included in both classes.

The hardness determinations by Talmage are given in part 3 of this bulletin. A few of the determinations there given differ from those of Talmage, owing to the fact that for these minerals he obtained his results on mislabeled specimens in the Murdoch suite. The writer, using Talmage's machine, determined the hardness of a number of minerals not found in that suite, and these results are included in the tables. It is believed that the Talmage hardness determinations af.

$\approx$ Vanderwilt, J. W., The nature of polished surfaces (doctor's thesis, Harvard Unt versity), p. 70,1927 . 
ford a mental picture of the relative hardness of different minerals, especially those in the "soft" class. Thus there would be no difficulty in getting a mental picture of the hardness of hessite (class A), which is about that of Talmage's type mineral, argentite, whereas millerite (class D) is about on the boundary between the "hard" and "soft" classes. The investigator, after some experience in scratching known minerals with an ordinary needle and looking up their Talmage hardness in the tables, will be able to subdivide minerals in the "soft" class roughly on the basis of hardness.

\section{EXAMINATION OF OPAQUE MINERALS IN POLARIZED LIGHT}

Summary.-The most useful test that can be applied to determine an unknown opaque mineral is the test for anisotropism. The test is made in less than a minute, and the result is highly definite and instructive. In this test a nicol prism is placed between the light source and the vertical illuminator of the metallographic microscope; after reflection from the polished surface, the light passes through another nicol prism whose planes of vibration are nearly or exactly at right angles with the planes of vibration of the polarizing prism. Isotropic minerals cause no change in the polarization direction of the light impinging upon them. Anisotropic minerals, on the other hand, rotate the plane of polarization of the incident light, and when the light passes the analyzer of the microscope interference effects are set up. Quantitative optical data corresponding to those obtained on transparent minerals by means of the petrographic microscope can not be obtained on opaque minerals. The amount of rotation produced can be measured but is only about $5^{\circ}$ for the mineral causing the greatest rotation; all other minerals show less than this amount. The results that are most valuable are qualitative. Minerals crystallizing in the isometric system and amorphous minerals are isotropic; minerals crystallizing in the other five crystal systems are anisotropic. Thus, a mineral can be quickly placed in one of two main classes by means of this test, and in the determinative tables here given this distinction is the first one made.

References.-The pioneer work on the effects of polarized light on opaque minerals is that of Koenigsberger. ${ }^{27}$ His apparatus makes use of only one nicol, the analyzer. Between the vertical illuminator and the analyzer is a Savart plate. He describes the anisotropism of a number of minerals.

\footnotetext{
${ }^{27}$ Koenigsberger, J., Ueber einen Apparat zur Erkennung und Messung optischer Anisotropie undurchsichtiger Substanzen und dessen Verwendung: Centralbl. Mineralogie, 1908, pp. 565-573, 597-605. (Translated in Winchell, N. H. and A. N., Elements of optical mineralogy, pp. 465-475, 1909.
} 
A mathematical discussion of the optical principles involved in Koenigsberger's apparatus is given by Wright, ${ }^{28}$ who describes cer. tain improvements in the apparatus and advocates the use of the Wright biquartz wedge in place of the Savart plate.

A greatly increased interest in the possibilities of the method was caused by the work of Schneiderhöhn. The apparatus that he devised is substantially that used by all subsequent investigators, including the present writer. A theoretical discussion of the phe. nomenon by Berek is given in Schneiderhöhn's text. ${ }^{29} \mathrm{~A}$ discus. sion of Schneiderhöhn's method, together with many original data, is given by Sampson. ${ }^{30}$ Some of Schneiderhöhn's theoretical con. clusions are criticized by Van der Veen. ${ }^{31}$ Excellent descriptions of the apparatus and directions for its use are given by Osborne: and by Fairbanks. ${ }^{33}$ The most recent and in many respects the most comprehensive description of the method is that of Sampson." A pparatus.-A petrographic microscope with an analyzing nicol in the barrel is the best instrument for investigating anisotropism. It is converted into a metallographic microscope by merely inserting a vertical illuminator between barrel and objective. The lower nicol of the petrographic microscope is not used in its original location but may be taken from its mounting and clamped in such a posi. tion that light passes horizontally through it and is polarized before reaching the vertical illuminator. It is preferable, however, to leave the lower nicol in its original place, in order to be able to change the instrument back to a petrographic microscope quickly. For a polarizer the writer uses a small nicol (made by E. Leitz) which is attached directly to the vertical illuminator and can be rotated. (See fig. 8.) A cap analyzer can be used instead of an inside analyzer. This form is required when the Wright biquartz wedge (p. 46) is used. The cap analyzer cuts down the field of vision and is very tiring to the eye, and its use is not recommended ex. cept where unavoidable. In the writer's instrument the inside an. alyzer is rotatable through an angle of $90^{\circ}$. This is more convenient than an analyzer fixed in position but is not absolutely necessary, as the same optical effect can be obtained by rotating the polarizing nicol. Sampson states that the plane of vibration of the polarizer

\footnotetext{
${ }^{28}$ Wright, F. E., Polarized light in the study of ores and metals: Am. Philos. Soc. Proc. vol. 58 , pp. $401-447,1919$.

so Schneiderhöhn, H., Anleitung zur microscopischen Bestimmung und Untersuchung von Erzen und Aufbereitungsprodukten besonders im auffallenden Licht, pp. 65-71, Berlin, 1922.

${ }^{30}$ Sampson, Edward, Note on the determination of anisotropism in metallic minerals: Econ. Geology, vol. 18, pp. 775-777, 1923.

${ }^{31}$ Van der Veen, J. W., Mineragraphy and ore deposition, pp. 32-36, The Hague, 1925.

32 Osborne, F. F., Technique in the investigation of iron ores: Econ. Geology, vol. 23, pp. $442-450,1928$.

${ }^{33}$ Fairbanks, E. E., The laboratory investigation of ores, pp. 24-27, New York, 1028.

" Sampson, Edward, The determination of anisotropism in metallic minerals: Econ. Geology, vol. 24, pp. 412-423, 1929.
} 
should be parallel or at right angles to the plane of the reflector of the vertical illuminator; if these planes are in an intermediate position serious disturbing effects are introduced. Ordinary light, such as is furnished by reflected daylight or the ordinary incandescent electric light, is inadequate for investigating opaque minerals in polarized light. A more intense illumination is needed, and a small arc lamp is very satisfactory for this purpose. The arc lamp used in the Harvard Laboratory of Economic Geology is made by E. Leitz and is known as the Lilliput arc lamp. (See fig. 8.) It is provided with a clockwork attachment for automatically feeding

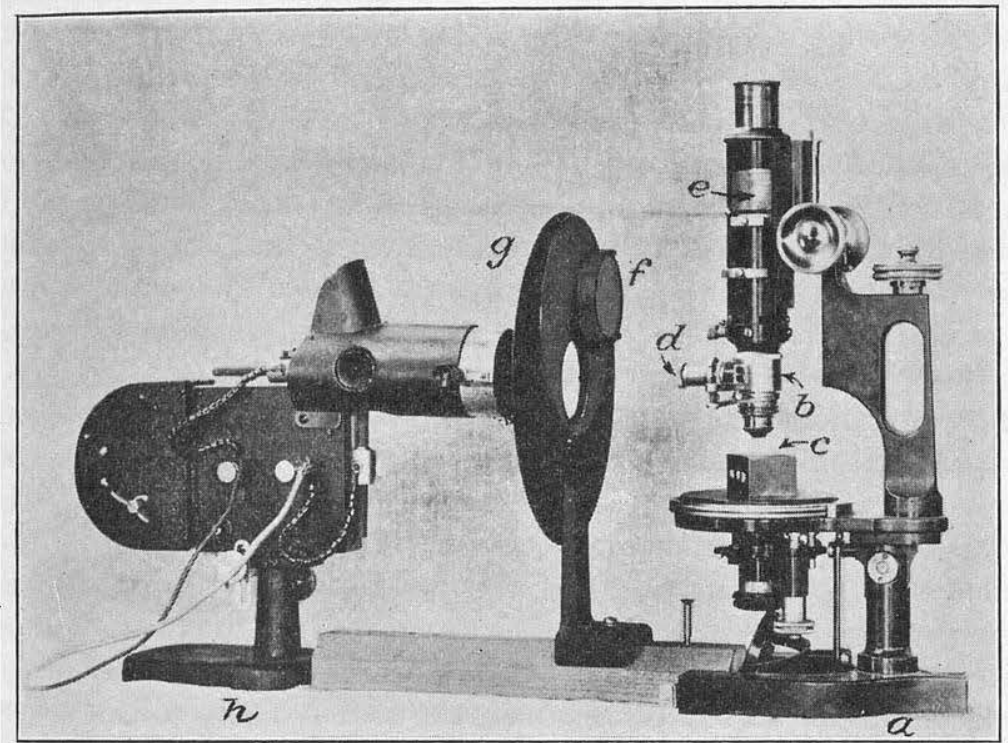

Figure 8.-Microscope arranged for use with polarized reflected light. I'hotographed by E. B. Dane, jr. $a$, Bausch \& Lomb petrographic microscope; $b$, vertical illuminator; $c$, polished section in brass mount; $d$, polarizer attached to vertical illuminator ; $e$, analyzer ; $f$, blue ground glass ; $g$, rotatable disk; $h$, small arc lamp

the carbon to the electric arc; this attachment is convenient but not necessary. A rheostat giving approximately $13 \mathrm{ohms}$ is necessary when using a lighting circuit of 110 volts. Direct current is superior to alternating current for this purpose.

When the analyzer is thrown out this light is too strong for the eyes, and a ground glass is placed between light and microscope. In the apparatus shown in Figure 8 this is placed on a rotatable disk by means of which it can quickly be placed in and out of the line of light.

The illumination unit furnished by Bausch \& Lomb consists of a 6-volt 108-watt Mazda tungsten-ribbon filament incandescent lamp with housing and a transformer for stepping the current down from 
110 volts. It is used with an alternating current. The principal advantage of the incandescent lamp over the arc lamp is the steadi. ness of the illumination. On the other hand, the 6-volt 108-watt incandescent lamp has only a short life-roughly 20 hours-and is expensive, whereas carbons for the arc lamp are relatively cheap. The writer uses the tungsten-ribbon lamp above described for microphotography.

The illumination unit used in the laboratory of the United States Geological Survey for investigating anisotropism is the Busch microscope lamp, made in Rathenow, Germany, and sold by the Palo Co., New York. The price of this lamp is approximately $\$ 21$. It is somewhat less expensive than the lamps already described and is highly satisfactory. It is furnished with a Mazda 110-volt 250 . watt projection bulb using either alternating or direct current. This bulb is less expensive than the 6-volt ribbon-filament bulb and has a longer life. It requires more manipulative skill, however, as the coiled filament gives a distinct image and the lamp must be shifted in such a way as to eliminate this image. This unit is almost identical with that devised by Prof. A. M. Bateman, of Yale University, and described by Osborne in the paper already cited.

The 16-millimeter short-mounted objective is by far the best for observations on opaque minerals in polarized light. The 24-millimeter and 8-millimeter objectives also give fair results but are in. ferior to the 16-millimeter objective. The 4-millimeter objective, which is the one most used for high magnification, is very unsatisfactory for this purpose, and with it only minerals of strong anisotropism exhibit the phenomenon. The writer can offer no explanation for this fact and has not seen it mentioned elsewhere in the literature.

Details of the method.-Opaque minerals belonging to the isometric system are isotropic when examined in the manner outlined above. If the polished section is turned $360^{\circ}$ by rotating the stage of the microscope, isometric minerals show no change of color or change of intensity of light during the revolution. Minerals of the other five crystal systems, on the other hand, are anisotropic when examined in the same manner. Some of them show four extinctions during the revolution, thus behaving like anisotropic transparent minerals examined in polarized transmitted light on the petrographic micro. scope. By far the greater number of anisotropic opaque minerals will give only two extinctions to a revolution. The minerals noted thus far that give four extinctions are covellite, umangite, manganite, pyrolusite, hausmannite, stibnite, and molybdenite. This list is incomplete, and probably a few more minerals will be added to it in the course of time. This property of giving four extinctions is of 
great diagnostic value because so small a proportion of the total number of opaque anistropic minerals exhibit it.

A few exceptions should be noted to the general rule that all isometric minerals are isotropic. Certain varieties of cobaltite exhibit weak anisotropism, as already noted by Schneiderhöhn and Sampson. The same is true of certain varieties of hessite, argentite, stannite, cuprite, pyrite, bornite, and perhaps a few others. Most specimens of these minerals are isotropic, however. A possible cause of the anisotropism may be the presence in small proportion of accessory elements which are not found in most specimens of the mineral. A more probable theory, however, is that these minerals may have been isometric at the temperature of formation but have undergone inversion to a lower symmetry during cooling. It is known that chalcocite and other minerals exhibit this phenomenon, as do also some transparent isometric minerals, notably leucite. Amorphous minurals are also isotropic. Very fine grained anisotropic minerals are sometimes apparently isotropic. For instance, specularite is moderately anisotropic, but earthy red hematite, which is probably not amorphous but minutely crystalline, appears isotropic.

Transparent minerals of high birefringence are anisotropic when examined in polished section, but those of low birefringence are apparently isotropic. The carbonate minerals can be identified at a glance and distinguished from quartz and other gangue minerals by this method. Light penetrates deeper into the transparent minerals, and only that which is reffected is visible. Small cracks and seams below the surface tend to disperse the light reflected and nullify the effects of polarization. In the determinative tables which follow it is noted that the ore minerals realgar and wurtzite are apparently isotropic.

The effect produced on polarized light by an anisotropic mineral is easily described. The nicols are first placed in the crossed position. This can be accomplished by observation on an isotropic mineral, such as galena. The analyzer is turned until the mineral appears darkest. If an anisotropic mineral is now observed it is found that the mineral is not in the position of extinction, but it will appear in that position if the analyzer is rotated a few degrees to the right or left. The anisotropic mineral has rotated the plane of polarization of the rays impinging upon it; rays striking the mineral with a "north-south" vibration are reflected with the direction of vibration at a small angle from that direction. The direction of vibration of the rays is no longer at right angles with the direction of vibration of the analyzer, and some of the light gets through. The angular rotation given to the analyzer to bring about extinction measures the angular rotation produced by the mineral. 
It is found that in a rotation of the stage the mineral rotates the polarization plane first to the right and after a $90^{\circ}$ rotation of the stage the polarization plane is rotated to the left; in a complete rotation of the stage there are two rotations to the right and two to the left.

As already stated, usually only two extinctions are observed, and even with a mineral showing four extinctions, such as molybdenite, two of them $180^{\circ}$ apart are sharp and clean-cut and the other two are less definite. This phenomenon, as Sampson points out, is due to absorption of light by the mineral; this absorption is stronger in certain directions than in others. It is analogous to pleochroism in a transparent mineral. Owing to this absorption, the positions of extinction during the revolution of the stage may be prolonged, and where the brightening of the mineral might otherwise have taken place, it is masked by absorption. However, if the Wright biquartz wedge plate is used all minerals will give four extinctions. for with this plate actual rotation is the effective agent, and this is not modified by the intensity of the reflected light.

Absorption is selective on rays of different wave length. Some are absorbed more than others, the degree of absorption depending on the mineral. Hence the color of the mineral as seen in polarized light is usually very different from its color as seen in ordinary light.

In minerals of strong anisotropism differently oriented grains have entirely different colors. For instance, arsenopyrite shoms colors similar to those shown by thin sections of hypersthene, rang. ing from pink to blue. However, such evidence should be accepted with caution. The polarization colors vary with the source of light (arc or incandescent), the intensity of illumination, and the perfer. tion of polish, in addition to the effects produced by the mineral itself. Owing to these factors the writer has not been able to check the descriptions given in Van der Veen's tables.

The amount of rotation produced by any particular anisotropic mineral ranges from zero to a maximum depending on the direction of the polished section with reference to the optical orientation of the mineral. Basal sections of hematite and molybdenite, for in. stance, are isotropic, whereas prismatic sections exhibit the greatest anisotropism for these minerals. Thus the maximum rotation of a mineral is a definite property of that mineral.

The measurement of such a constant, however, is attended with great difficulty, because the position is not clean-cut like that of a transparent mineral but is wavy and is thus harder to locate. To obtain this position a Wright biquartz wedge is necessary, and even with this a series of observations is required in order to obtain 
the exact angle by the method of averages. This must be extended to a number of grains, as the rotation of the polarization plane produced by the mineral depends on the orientation of the grain and thus varies in the different grains cut by a polished section. On the other hand it is a simple matter to pick out the grains giving the greatest rotation, by simply noting those that show the greatest contrast between light and dark positions as the stage is rotated.

In addition to the difficulty in obtaining the maximum angle of rotation of the plane of the polarization for any given mineral, there is the added objection that this angle, even if constant for any mineral, varies only a few degrees between the minerals with greatest polarization effect and those with least. Chalcopyrite, whose anisotropism is barely discernible, gives a rotation of a small fraction of a degree, whereas covellite, which has a greater polarization than any other mineral yet observed, rotates the angle only about $5^{\circ}$. The others are covered by this small range. Owing to these difficulties, the writer does not believe that it is practicable to use the maximum rotation as a constant to determine a mineral, as the maximum extinction perpendicular to " $m$ " is used to determine a feldspar; but minerals can be subdivided readily into two groups, isotropic and anisotropic, and the latter can be subdivided by such general terms as weakly, moderately, and strongly anisotropic-not exact terms, to be sure, but useful hints.

Methods of observation.$^{35}$-Anisotropism may be detected in three ways-(1) with the analyzer in the crossed position; (2) with the analyzer slightly rotated from the crossed position; and (3) by the use of the Wright biquartz wedge and nicol.

The method with the analyzer crossed is the least sensitive of the three. As the stage is rotated, a strongly acting mineral will change from complete darkness to a very faint illumination.

If the analyzer is placed so that it is not exactly in the crossed position, the anisotropic effect can be seen better, particularly where several contiguous grains of the mineral lie in the same field. With the analyzer thus turned, the mineral when in the four positions of no rotative effect will be in extinction, as if it were isotropic; but when in the position of greatest rotative effect it will be brighter or darker than it would have appeared had the analyzer not been turned, because the mineral by its rotative effect is adding to or subtracting from the effect of the turned analyzer. The advantage of this method of observation is similar to that obtained by using a gypsum plate with a petrographic microscope to secure a sensitive tint. The eye can better detect changes in the intensity of a mod-

\footnotetext{
s This description of the three ways of detecting anisotropism is taken almost verbatim from Sampson's article (Econ. Geology, vol. 24, pp. 415-418, 1929).
} 
erate brightness than in that of almost complete darkness. In a polished section that shows several grains of a mineral the effect of anisotropism is readily seen, for one grain may be in the position of least illumination while its neighbor may be in the position of greatest brightness.

An effect similar to that caused in a granular aggregate is pro. duced in a single grain by using a Wright biquartz wedge plate with the analyzer in the exactly crossed position. This compares two moderately bright fields. The plate contains two sections of quartz cut parallel to the base. One section is from a right-hand crystal, the other from a left. They are so mounted that the line separating them is a medial line in the microscope field. Half of the plate rotates light to the right, and half to the left. The combined quart? sections are ground to a wedge, so that the degree of rotation varies according to the thickness of the part of the plate used. The Wright wedge is used with a cap nicol. If light reflected up the microscope tube is plane-polarized, and the plane of polarization corresponds to the zero setting of the cap nicol, the two halves of the field will be equally illuminated. If, however, the reflected light has its plane of polarization turned from this zero position, as may be brought about by an anisotropic mineral, the intensity of illumination on opposite sides of the plate will vary according to whether the ro. tation caused by that side of the plate is the same as or opposite to that caused by the mineral. If the cap nicol is now rotated an amount equal to the rotation caused by the mineral, the illumination will again be equal. By this method the amount of rotation of the plane of polarization caused by the mineral may be measured quanti. tatively. However, the Wright biquartz wedge is useful principally for refined qualitative determinations, particularly when such determinations must be made on an isolated grain; with such a grain the effect of a slight change in the intensity of illumination is aug. mented by using the Wright plate, because the effect of the reflected light adds to the brightness on one side of the plate and detracts from that of the other side, according to whether the rotation caused by the mineral is the same as or opposite to that of the piece of rotating quartz in each half of the plate.

Adjustment of the instrument.-The writer uses the second method described above exclusively. The Wright wedge is an effective method of determining anisotropism, but the use of the cap nicol is a serious handicap. The second method gives results equally good, permits the use of a tube analyzer, and dispenses with a change of ocular.

The question naturally arises as to how far from the crossed position the analyzer (or polarizer) should be turned. The writer finds 
a polished section of chalcopyrite from Sudbury, Ontario, of value in getting the best adjustment of nicols. This chalcopyrite shows multiple twinning similar to that of plagioclase feldspar, but the anisotropism is very feeble. The nicol (either polarizer or analyzer) is turned until there is a maximum color difference between adjacent lamellae. The darker lamellae will have a color about the same as that of bornite in ordinary light, and the lighter lamellae will be greenish yellow. This adjustment is found to be satisfactory for all anisotropic minerals. Figure 9 shows chalcopyrite that gives an effect identical with that of the Sudbury mineral.

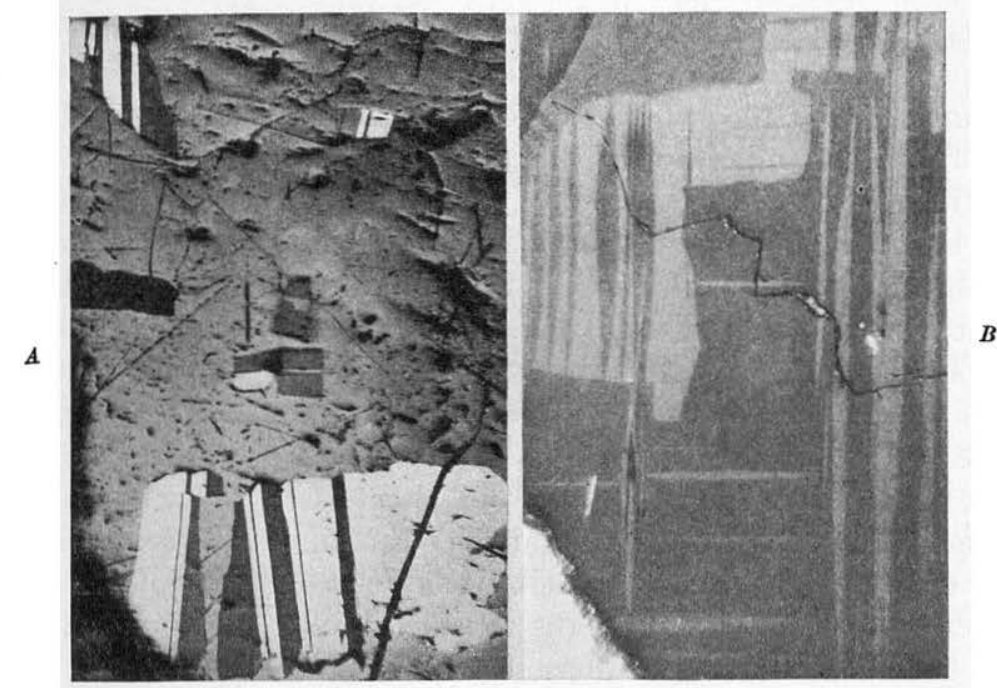

Figure 9.-Photomicrographs of ores taken in polarized reflected light. (After Sampson. Courtesy of Economic Geology.) A, Marcasite, showing polysynthetic twinning, in pyrite. Nicols nearly crossed. From material collected by A. W. Stickney and studied by him in the laboratories of the United States Geological Survey. This plate also illustrates that an imperfect polish does not interfere with clear-cut qualitative results. $\times 60 . B$, Twinning in chalcopyrite from Bass prospects, Grand Canyon, Ariz. Graín of pyrite in upper left corner. Nicols nearly crossed. Specimen unetched. $\times 150$.

Experience in teaching the subject demonstrates that making this adjustment is by far the most difficult task that the student will encounter. Sufficient practice alone will overcome this difficulty. The nicols are first set in approximately the crossed position. The polarizer and vertical illuminator are then pointed as directly at the light source as possible. The chalcopyrite surface is then observed through the microscope while one of the nicols is turned through a few degrees one way and then the other. If the appearance of the chalcopyrite lamellae is not satisfactory, the polarizer and vertical illuminator are swung a little to either side on the vertical axis of 
the illuminator, and the same process is repeated. This is continued until the chalcopyrite lamellae show the maximum contrast. Then the lamp is shifted to one side or the other until the illumination as seen in the microscopic image is evenly distributed. After some experience a student of average ability can make this adjustment in five minutes or less.

Sources of error.-The surface of the mineral must be at right angles to the axis of the microscope-that is, parallel to the stageotherwise there is a variation in the intensity of the light as the see. tion is rotated. In a complete rotation of the stage the section will be tilted toward the light once and away from it once if the section and stage are not parallel. If the section is tilted toward the lamp. that part of the field of view toward the lamp is more strongly illuminated, and the other part is shaded. If the section is tilted away from the lamp (toward the observer), the reverse is true. This variation in intensity resembles the extinctions of anisotropic min. erals, but it may be distinguished from anisotropism by the fact that there is only one "extinction" to the revolution and also by the fad that it can be observed with the analyzer out. If the polished see. tions are mounted on modeling wax they can be easily adjusted bf means of the leveling cup (see p. 7) so that the surface is paralle to the stage. When the specimens are solidly mounted in bras boxes, the top and bottom of each box should be ground parallel.

If more than one grain of an anisotropic mineral is in the field of view, the above-mentioned possibility of error is obviated, because differently oriented grains of the mineral have sharp boundaries and show different colors or different shades of the same color under crossed nicols. Fortunately, most opaque minerals are fine grainedthat is, the size of individual grains is smaller than the field of view of the 16-millimeter objective, which is approximately 2 milli. meters in diameter.

Poor polishing and irregularities of relief also lead to error in interpretation of polarization effects. This is particularly true of the hard minerals, such as chloanthite and other arsenides. Rotation of the stage gives rise to vague irregularities in illumination which the beginner is wont to interpret as anisotropism. Experience alone will enable him to discount these effects. Above all, grain boundaries should be looked for.

The causes of failure in the investigation of anisotropism are ladk of adequate illumination, poor adjustment of the nicols, and pseudo. extinctions due to lack of parallelism between polished surface and microscope stage or to irregularities of relief on the polished surface. 


\section{ELECTROCONDUCTIVITY}

Methods for determining the electrical conductivity of minerals have been described by Farnham, ${ }^{36}$ by Kerr and Cabeen, ${ }^{37}$ and by Harvey. ${ }^{38}$ The writer has not had any experience with these methods, but they promise to be of considerable assistance in the identification of the ore minerals. It appears from Harvey's work that the conductivity in some minerals varies enormously in different specimens, and this factor weakens the effectiveness of the method. According to Harvey, the principal cause of this variation lies in slight differences in chemical composition of the mineral.

${ }^{*}$ Davy, WV. M., and Farnham, C. M., op. cit., p. 8.

${ }^{37}$ Kerr, P. F., and Cabeen, C. K., Electrical conductivity of the ore minerals: Econ. Geology, vol. 20, pp. 729-737, 1925.

${ }^{s s}$ Harvey, R. D.. Electrical conductivity and polished sections: Econ. Geology, vol. 23, p. $778,1928$. 


\section{Part 3. ETCH REACTIONS AND DETERMINATIVE TABLI}

\section{ETCH REACTIONS}

The term "microchemical test" is used both by Murdoch and br Davy and Farnham to describe the etching effects produced by nitrie acid and other reagents on the polished surface of the mineral to be investigated. It is believed that the term " etch test," used by Schne. derhöhn and by Van der Veen, is preferable. The terms "micro. chemistry" and "microchemical test" have been used since 1877 to designate qualitative microscopic tests or reactions for elements The tests are usually made on a glass slide. To avoid confusion, the term " microchemical test" will be confined in this bulle. tin to qualitative chemical tests made on a glass slide; tests made directly on the polished section will be referred to as "etch tests."

The etching effects or lack of effects of a set of chemical reagents of standard strength have been the principal means relied upon br all the above-named men to identify the unknown opaque minerals.

Murdoch "pigeonholes" the minerals according to their reactivity toward the following solutions: $\mathrm{HNO}_{3}, 1: 1 ; \mathrm{HNO}_{3}$, concentrated: aqua regia; $\mathrm{HCl}, 1: 1 ; \mathrm{HCl}$, concentrated; $\mathrm{KCN}, 20$ per cent; $\mathrm{FeCl}_{\text {, }}$ 20 per cent; $\mathrm{KOH}$, saturated solution. In his descriptions of the individual minerals, their reactions with the following solutions are also given: $\mathrm{NH}_{4} \mathrm{OH}, \mathrm{NaOH}$, iodine, $\left(\mathrm{NH}_{4}\right)_{2} \mathrm{~S}_{\times}, \mathrm{K}_{4} \mathrm{Fe}(\mathrm{CN})$ o $\mathrm{K}_{3} \mathrm{Fe}(\mathrm{CN})_{6}, \mathrm{H}_{2} \mathrm{SO}_{4}, \mathrm{AgNO}_{3}, \mathrm{KClO}_{3}$. Not all these reagents were used on any one mineral.

Davy and Farnham greatly simplified the scheme by using onl? four reagents to "pigeonhole" the minerals. These are $\mathrm{HNO}_{2}$ $1: 1 ; \mathrm{HCl}, 1: 1 ; \mathrm{KCN}, 20$ per cent; and $\mathrm{FeCl}_{3}, 20$ per cent. In their detailed descriptions of the minerals the reactions produced br saturated solutions of $\mathrm{KOH}$ and $\mathrm{HgCl}_{2}$ are also given.

Both Schneiderhöhn and Van der Veen have added a number of reagents to those already mentioned. Neither made any at. tempt at a systematic classification of minerals according to their reactivity toward chemical reagents, but both laid emphasis on reagents that bring out twinning and other internal structures in the mineral.

There are some strong objections against relying too much on? etch behavior in identifying a mineral. The greatest trouble with the scheme is that some reactions are very feeble. Many of then. indeed, are so feeble that they will work sometimes and fail to work 
at other times, even on the same specimen. It is known that the susceptibility to etching is different in different orientations of the same mineral specimen, and orientation alone may be the chief factor in determining whether or not a reagent will etch a specimen. Slight differences in chemical composition of a mineral may cause some specimens to etch, whereas other specimens may be entirely negative to the same reagent. These variations were recognized specifically in the text of Davy and Farnham and in that of Van der Veen, but in the former the variations are not sufficiently considered, and the authors did not make the tests on a sufficient number of specimens of each mineral to reveal all the variations.

It is obvious that a mistake in interpreting any one reaction will cause the determinative scheme to fall down completely. When a reaction is weak some difficulty is experienced in deciding whether or not it is positive. The only solution of these difficulties is to place a mineral that is sometimes etched by a reagent and is sometimes negative toward it in both the "positive reaction" and "negative reaction" classes. For instance, tetrahedrite is sometimes etched by 1:1 $\mathrm{HNO}_{3}$ and is sometimes inert toward it. It should be placed in both the "etched by $\mathrm{HNO}_{3}$ " and "negative to $\mathrm{HNO}_{3}$ " groups. Sphalerite is usually negative to $1: 1 \mathrm{HCl}$, but one or two specimens that gave faint reactions with $\mathrm{HCl}$ have been observed. These facts should be noted in describing sphalerite, and it should be placed in both the "etched by $\mathrm{HCl}$ " and "negative to $\mathrm{HCl}$ " groups.

When two different minerals are covered by a drop of a reagent, an electric current is generated owing to the difference of potential between the minerals. As a result, the effect of the reagent is intensified on one of the minerals and diminished on the other. For instance, when bornite and galena in contact are covered with ferric chloride solution, the bornite reaction is stronger than it is with pure bornite alone; on the other hand, the galena adjacent to the bornite is entirely negative to ferric chloride solution, whereas pure galena alone gives a strong reaction with this solution. It is therefore advisable to find an area of the mineral larger than the drop to be applied and to prevent the drop from spreading over the contact with an adjacent mineral. It is not always possible to do this, and under such conditions a knowledge of the electrolytic effects produced by the different mineral associations is advisable, in order to discount them in identifying the mineral. Fortunately in the great majority of mineral associations these electrolytic effects are too feeble to affect the etch reactions.

The manner of applying the reagents to the polished surface differs considerably. Schneiderhörn immerses the entire polished 
surface face down in a receptacle containing the reagent. In this manner the entire section is acted upon by the etching reagent, and electrolytic effects can not be avoided. In addition, the entire surface of the specimen must be repolished before other tests can be made.

Murdoch applies a drop of reagent directly to the polished surface by means of a small capillary pipette. This procedure is also fol. lowed by Davy and Farnham. The objections to the capillary tube are that it is fragile and easily broken, the ragged end tends to scratch the specimen, and the amount of liquid transferred is not easily controlled.

The procedure recommended by the writer is that of Prof. L. C. Graton, who uses a platinum wire with a small loop on one end of it. The other end of the wire is held by a glass rod or other suit. able holder. The diameter of the wire used by the writer is 0.32 millimeter, the distance from the end of the wire holder to the end of the loop is 20 millimeters, and the inside diameter of the loop is 0.41 millimeter. The wire is bent with the loop at an angle to the rest of the wire, forming a tiny golf club, as shown in Fig. ure 10. The loop is filled with reagent by means of a glass dropping rod, and with the eye to the microscope the loop is brought into the field of view and slowly

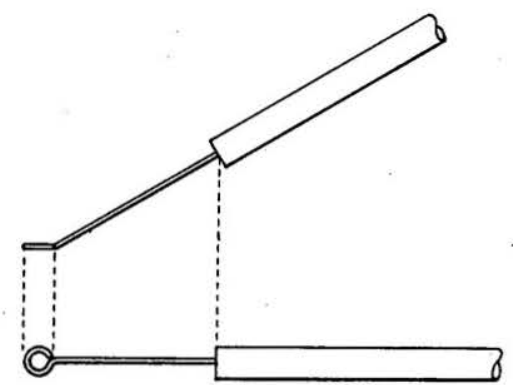

Figure 10.-Platinum' wire for transferring drop of reagent to polished surface. Plane of loop is at an angle to rest of wire lowered until the liquid comes into contact with the mineral. The liq. uid remains on the mineral when the loop is raised.

Van der Veen places the drop on the specimen and leaves it there one minute, then washes the reagent off, and dries the specimen before it is placed under the mi. croscope. He does not believe in microscopic observations on the specimen during etching, on the ground that fumes given off by

the reagents are injurious to the microscope, especially the objective. The injurious effect of the reagent fumes has probably been overesti. mated by Van der Veen. The writer has used the same microscope objective in this work for three years and can observe no harmful effects.

If the specimen is observed by means of the microscope during etching, the position of the drop can be carefully controlled, and the specimen can be removed and washer before the etching has proceeded too far. For instance, the etch cleavage of chalcocite is brought out by KCN solution, but if etching is continued too long the etch structure is entirely destroyed and the surface turns black. 
The 16-millimeter objective is used for observation of the application of the drop and the etching. It is not advisable to use an objective of higher magnification when applying the etching liquids, as there is danger of the objective coming into contact with corrosive fluids. The fluid should be removed and the specimen washed before changing to objectives of higher magnification.

The time during which etching is continued varies with the mineral. Some minerals tarnish instantly with certain reagents, and the etching fluid can be removed immediately. The time limit set by the writer for the slower reactions is usually one minute. If no effect can be observed at the end of one minute the test is considered negative. If the mineral is not completely etched at the end of one minute, but some change is noticed on the surface, sufficient time is given to allow the effect to be completed. An exception is made of $\mathrm{HNO}_{3}$. With some minerals like jamesonite the etching with this reagent is sometimes slow in starting, but once started it proceeds rapidly. For this reason, if the $\mathrm{HNO}_{3}$ drop still has a negative effect at the end of one minute, it is preferably allowed to remain on the surface until it begins to dry up, or approximately two minutes.

The reagents recommended by Davy and Farnham are on the whole satisfactory and are therefore used by the writer. Adding to the number of reagents merely adds to the difficulties already mentioned without any compensating advantages. Any reagent used will strongly etch some minerals but will have no effect on others, and there is a group of minerals on which the effect will be between these extremes and with many of them weak and indefinite. The reagent recommended by Schneiderhöhn, $\mathrm{KMnO}_{4}$ in $\mathrm{H}_{2} \mathrm{SO}_{4}$, is effective in bringing out structures in certain minerals, but it has the disadvantage just noted and in addition is messy and has to be made up fresh every few days. It is therefore not recommended for routine determinative etch tests.

Of the reagents used by Davy and Farnham, $\mathrm{HNO}_{3}$ and $\mathrm{HCl}$ leave no residues on evaporation. $\mathrm{KCN}, \mathrm{FeCl}_{3}, \mathrm{KOH}$, and $\mathrm{HgCl}_{2}$, being dissolved salts, will each leave a residue on evaporation, and this sometimes forms an iridescent scum which is closely similar to an etch tarnish. Owing to this difficulty it is not advisable to allow the reagent to evaporate to a point where it begins to drop its dissolved load. Consequently it is recommended that the saturated $\mathrm{KOH}$ solution be changed to a 40 per cent solution. Mercuric chloride is more troublesome. Davy and Farnham recommend a saturated solution (approximately 7 per cent at ordinary temperatures). In a saturated solution mercurous chloride separates out, as well as mercuric chloride. Mercurous chloride tends to stick to the surface of a mineral and is hard to remove. Beginners especially are $15122-31-5$ 
liable to confuse this mercuric chloride precipitate with an etch reaction. For this reason the writer recommends a 5 per cent solu. tion of mercuric chloride ( 1 gram of $\mathrm{HgCl}_{2}$ with sufficient water added to make 20 cubic centimeters of solution). Even with this concentration some mercurous chloride will slowly separate out, and it is advisable to filter the solution from time to time.

The reagents used by the writer are as follows:

$\mathrm{HNO}_{3}, 1: 1$ ( 1 part $\mathrm{HNO}_{3}$, specific gravity 1.42, to 1 part $\mathrm{H}_{2} \mathrm{O}$ ).

$\mathrm{HCl}, 1: 1$ ( 1 part $\mathrm{HCl}$, specific gravity 1.19 , to 1 part $\mathrm{H}_{2} \mathrm{O}$ ).

KCN, 20 per cent solution.

$\mathrm{FeCl}_{3}, 20$ per cent solution.

KOH, 40 per cent solution.

$\mathrm{HgCl}_{2}, 5$ per cent solution.

Aqua regia (3 parts concentrated $\mathrm{HCl}$ to 1 part concentrated $\mathrm{HNO}_{3}$ ).

$\mathrm{H}_{2} \mathrm{O}_{2}$, commercial reagent.

Aqua regia is used only for those minerals which are negative to the other solutions. The strength of the acids should be checked from time to time by means of a specific-gravity hydrometer.

The researches of Thiel have shown that most of the manganese oxide minerals break down hydrogen peroxide, $\mathrm{H}_{2} \mathrm{O}_{2}$. A drop of the commercial reagent placed on the mineral effervesces, but the mineral itself is not etched. ${ }^{39}$ According to Smitheringale, ${ }^{40}$ these reactions are less positive than indicated in Thiel's paper. The present writer checked Thiel's results rather closely and found that if anything the reactions are even more positive than indicated by Thiel. Evidently the quality of the reagent is a vital factor in the intensity of the test. The author uses the 3 per cent solution manufactured by the Oakland Chemical Co., New York. It is used only to confirm manganese oxides.

It is advisable to keep the $\mathrm{HNO}_{3}$ and $\mathrm{HCl}$ solutions in glass-stoppered dropping bottles similar to those used for refractive-index oils, as rubber stoppers are corroded by the acids. The other four solutions, on the other hand, should not be kept in glass-stoppered bottles, as the liquid tends to evaporate, and the solid residue cements the stopper to the bottle. $\mathrm{KOH}$ will often form so tight a cement that the bottle will be broken in trying to remove the stopper. For the salt solutions other than the acids rubber stoppers are recommended. The stopper has a hole punched in it for the purpose of holding a glass dropping rod 4 millimeters in diameter. All the reagent bottles have a capacity of 15 cubic centimeters.

Minerals that effervesce when acted upon by $1: 1 \mathrm{HNO}_{3}$ are separated from those that are etched but do not effervesce. This is a

${ }^{*}$ Thlel, G. A., The manganese minerals; their identiflcation and paragenesis: Econ: Geology, vol. 19, p, 107, 1924.

${ }^{40}$ Smitheringale, W. V., Etching tests and X-ray examinations of some manganese minerạls : Ẹcoṇ. Geology, vol, 24, p, 481, 1929. 
useful distinction that is made both by Murdoch and by Davy and Farnham and is retained by the writer. For instance, niccolite and breithauptite, which otherwise can not be distinguished except by chemical tests, are separated by the fact that niccolite effervesces with $\mathrm{HNO}_{3}$ and breithauptite tarnishes without effervescence. The great difficulty with effervescence as a criterion is that the effervescence is sometimes very weak and apt to be overlooked. For instance, pyrite will give a slow effervescence, a few bubbles at a time, if the surface is smooth and well polished, but usually the surface is rough and the grains small, and under these circumstances effervescence generally does not take place. Here again such a mineral may be placed in both the "tarnishes with effervescence" and "tarnishes without effervescence" classes.

The effect of calcite and other carbonates must be watched for. When a carbonate is present as a gangue mineral a vigorous effervescence produced by acids is often mistaken for a reaction on an adjacent ore mineral. This can be discounted by noting that the ore mineral is not tarnished. When an area on a polished section shows efferveseence with both $\mathrm{HNO}_{3}$ and $\mathrm{HCl}$, the presence of calcite should be suspected.

A fountain-pen filler is useful for removing a reagent drop from the specimen. The end of the filler is heated and drawn out to a point and broken off, giving a tube of small diameter. This is filled with water. When the etch reaction is complete, the polished section is removed from the microscope, and by means of the fountain-pen filler, a stream of water is squirted on that part of the surface of the section just etched, and the reagent drop is washed off. With care, only part of the surface need be wetted. The polished section is again brought under the microscope, and the area formerly covered by the etch drop is examined while still wet. The etch test is now complete, and the information is recorded.

The surface is then dried by means of a filter paper and rubbed on a chamois cloth which is mounted on a block of wood. Very fine floated rouge should be sprinkled on the surface of the rubbing block. (See p. 13.) The unknown mineral is then ready for the next etch test. The writer does not recommend examining the section after rubbing on the block, as recommended by Murdoch and by Davy and Farnham. In both books are to be found descriptions like the following: "Stains brown; rubs to a gray roughened surface." It has been found that the character of the surface after rubbing varies too much to be of use in identification tests. The rouge has an abrading action and if continued long enough would remove the etching from any surface.

In addition, some remnants of the reagents are likely to remain on the surface, even after washing. The reagent may have soaked 
into the cracks, pits, and pore spaces; or the residue after evapor. tion may adhere to the surface itself and remain there in spite of continued efforts to wash it off. Blotting and rubbing on a block only spread this residue over the surface, giving an iridescent film which, as experience has shown, is often mistaken for a positive etch reaction.

The safest and most satisfactory method of making an etch test is to observe it microscopically from the time the drop of reagent is applied to the surface until the section is removed for the purpose of washing the reagent off. The effect can be observed right through the drop with all reagents except ferric chloride solution, This solution has a dense yellow color which may obscure the color of the etched surface. The effect of the ferric chloride can only be observed after washing it off, shaking the section to remove the ex. cess water from the surface, and observing the section through the water remaining on its surface. It is never advisable to blot the specimen or to dry it before making the final microscopic observa. tion. The water itself usually contains enough dissolved matter to smear a film over the surface when dried. This smear can be re moved only by rubbing it on the rouged chamois.

The character of the etching is well described in all the text books cited. Generally the effect is merely a discoloration of the surface. Where the etching action is strong the surface may bo corroded, becoming rough or full of pits. With many minerals, scratches formed during polishing and invisible before etching are brought out by the etching process, owing to the fact that the scratches are widened by solution of the mineral.

With some minerals, notably chalcocite, etching brings out a series of closely spaced parallel cracks. This is known as "etch cleavage." Etch cleavage indicates the direction of greatest solution, and this does not necessarily indicate the direction of least molecular cohesion. For instance, the etch cleavage of orthorhombic chalcocite is paral. lel to the base, whereas the cohesion cleavage is parallel to the prism. In most specimens the etch cleavage probably indicates twinning planes. These are planes of structural weakness and are readily widened by the access of the reagents. ${ }^{41}$ In some specimens- 0 f cobaltite, for example-these cracks indicate the boundaries between shells in zoned crystals.

The intensity of etching differs in different directions of a crystal. A random polished section cuts different grains of the same mineral in different crystallographic directions; consequently, when a pol. ished surface is etched, adjacent grains will show different colors after etching. This effect is extremely useful in bringing our min:

1 Dr. Paul F. Kerr in a personal communication to the writer suggests that for main specimens the term "etch cleavage" is inaccurate and that where twinning planes ar followed the term " etch parting" would be preferable. 
eral-grain patterns. In addition to this differential etching, the spaces between adjacent grains are widened by solution, and spaces that were submicroscopic before etching may become visible after it.

With some minerals, certain reagents have no effect on the surface covered by the drop, but fumes from the liquid tarnish the area immediately contiguous to the periphery of the drop, thus forming an aureole around it. Usually this halo is soluble in water and disappears on washing the specimen. Under some conditions, however, it is permanent and thus becomes of additional diagnostic value. For instance, some silver minerals such as argentite and stephanite will form an insoluble aureole when etched by $1: 1 \mathrm{HCl}$, and this aureole is diagnostic of silver. These aureoles have been ignored by some authors and used by others. The present writer considers that a test resulting in an aureole is positive. Some nicety of judgment is required when an aureole is faint or when it does not entirely surround a drop. This is usually due to different orientation of adjacent grains. Such difficulties are unavoidable; the etching scheme is not perfect, and unless the effects are distinctive the mineral must be checked by other properties.

Some minerals show nothing but a halo of small drops of liquid adjacent to the main drop. These might be termed "sweat halos." They are ignored in the tables of etch reactions.

Probably the greatest source of error in making the etch tests arises from not having a clean surface to begin with. Some minerals like bornite tarnish very quickly, and with such minerals consistent results can not be expected. It is remarkable how quickly a specimen can acquire a slight film of dirt or grease from contact with other objects. This film hinders a good contact ibetween polished surface and liquid and may cause an otherwise positive reaction to fail. The obvious remedy is to make sure that any such film is removed before applying the reagent. The rouge block is usually effective in removing a film of dirt or grease, but it may fail to remove an oxidized tarnish unless rubbing is continued for some time. This rubbing, if continued, is certain to scratch the softer minerals. All laboratories where determinative work on the opaque ore minerals is carried on should be equipped with a burnishing wheel, using wet magnesia or black magnetic rouge on a billiard cloth. (See p. 12.) Such a wheel can readily be improvised from an electric fan by removing the propeller blades and mounting a wooden or steel disk on the shaft. By holding the specimen a few seconds on such a wheel the specimen is freed of any oxidized tarnish, grease film, or light etching. This wheel, however, is insufficient to remove a deep corrosion on the surface of the specimen, such as that made on galena by $\mathrm{HNO}_{3}$, which usually requires regrinding the surface on a steel lap. 
Although the systematic etching scheme just described has many disadvantages and can not be relied upon by a beginner, it is highly useful owing to the rapidity with which the tests can be made and the number of groups or "pigeonholes" that can be formed from a few reagents. A series of six etch tests can be completed, allowing for duplications of tests in doubtful reactions, in about $20 \mathrm{~min}$ utes. The ambiguities of etch behavior can for the most part be tabulated by a sufficient number of observations on specimens of the same mineral from different localities. The error resulting from mistaking a residue from the evaporation of a reagent for an etch tarnish can be avoided after a little experience.

It must be remembered that no set of tables of etch reactions will ever be perfect, however carefully they are made. Ninety-nine specimens of galena may be etched by $\mathrm{HNO}_{3}$, and the hundredth will not etch even after it is carefully burnished. Therefore, the etch tables can not be relied upon absolutely for the final determination of a mineral, and a determination made by systematic etching must be checked by other properties such as color, hardness, polarization or microchemical tests, or all of these.

\section{DETERMINATIVE TABLE}

\section{INTRODUCTION}

In the following determinative table the minerals are divided into two principal classes-soft and hard-and each class is subdivided into isotropic and anisotropic. The soft minerals are given on pages 71-96 and the hard minerals on pages 97-104.

In each of the main groups the minerals are further divided into "pigeonholes" by means of their etch reactions. The arrangement of these "pigeonholes" is the same as that of Davy and Farnham. The three principal subdivisions are based on the reactivity of the minerals toward nitric acid, as follows: (1) Effervesces with $\mathrm{HNO}_{3}$; (2) reacts with $\mathrm{HNO}_{3}$ without effervescence; (3) does not react with $\mathrm{HNO}_{3}$. Each of these subdivisions is further subdivided by reactivity toward $\mathrm{HCl}, \mathrm{KCN}$, and $\mathrm{FeCl}_{3}$, in order.

An outline of the scheme is given on pages 65-70. In this outline the subdivisions based on reactivity toward nitric acid are indicated in the columns farthest to the left on each page. They are divided by means of horizontal lines extending entirely across the page. The subdivisions based on the remaining reagents are similarly arranged. The chemical symbol of the reagent alone, without further designation, indicates that the etch test is positive. If the reaction is negative, the abbreviation " neg." is given with the chemical symbol. An inspection of the outline will show the systematic way in which the "pigeonholes" are arranged. In the detailed descriptions the reactions to $\mathrm{KOH}$ and $\mathrm{HgCl}_{2}$ are also given. 
The following examples will illustrate the use of the tables:

1. The mineral is soft, anisotropic; color, galena-white; etch reactions, $\mathrm{HNO}_{3}$ effervesces, $\mathrm{HCl}$ negative, $\mathrm{KCN}$ negative, $\mathrm{FeCl}_{3}$ stains light brown, $\mathrm{KOH}$ negative, $\mathrm{HgCl}_{2}$ negative. Reference to the tables shows that the mineral falls into the group on pages 76-77. The description fits any mineral in the group except calaverite, and if the observer's color perception is not good he might assume that it fits calaverite as well. To determine the mineral microchemical tests are necessary. It will also be seen that every mineral in that group contains arsenic, bismuth, or tellurium, hence they are the first elements to be tested for. Tests are made for other elements if necessary.

2. The mineral is soft, strongly anisotropic; gray in vertically reflected light; etch reactions, $\mathrm{HNO}_{3}$ negative, $\mathrm{HCl}$ fumes tarnish faintly, $\mathrm{KCN}$ stains brown, $\mathrm{FeCl}_{3}$ negative, $\mathrm{KOH}$ stains iridescent, $\mathrm{HgCl}_{2}$ negative. The corresponding pigeonhole in the tables is the group on page 91 , and the mineral is either stephanite or miargyrite. Scratching the mineral with a chisel-pointed needle (see p. 119) yields a ruby-red powder, which determines the mineral as miargyrite, therefore no microchemical tests are necessary. The $\mathrm{HCl}$ reaction is evidently doubtful, and on the assumption that it is negative the mineral will be found in the group on page 91 . Miargyrite is one of the minerals in that group, thus affording further confirmatory evidence.

The formulas given for minerals in the determinative table are those of Wherry and Foshag. ${ }^{42}$

2 Wherry, E. T., The nomenclature and classiflcation of sulfide minerals: Washington Acad. Sci. Jour., vol. 10, pp. 489-496, 1920 . Wherry, E. T., and Foshag, W. F., A new classification of the sulfo-salt minerals: Idem, vol. 11, pp. 2-8, 1921. 



\section{INDEX}

[Minerals marked * are discredited or doubtful]

Page

80,86

Aguilarite, $\mathrm{Ag}_{2}(\mathrm{Se}, \mathrm{S})$

$76,78,87,89$

Aikinite, $\mathrm{Cu}_{2} \mathrm{~S} .2 \mathrm{PbS} . \mathrm{Bi}_{2} \mathrm{~S}_{3}$

74

Alabandite, $\mathrm{MnS}$.

Alaskaite, $\mathrm{Ag}_{2} \mathrm{~S} . \mathrm{PbS}_{2} 2 \mathrm{Bi}_{2} \mathrm{~S}_{3} \ldots 1,87$

Algodonite, $\mathrm{Cu}_{0} \mathrm{As} \ldots \mathrm{C}$
Altaite, $\mathrm{PbTe}$

Andorite, $\mathrm{Ag}_{2} \mathrm{~S} .2 \mathrm{PbS}_{3} 3 \mathrm{Sb}_{2} \mathrm{~S}_{3} \ldots$

Antimony, Sb . .

Aramayoite, $\mathrm{Ag}_{2} \mathrm{~S}$. $(\mathrm{Sb}, \mathrm{Bi})_{2} \mathrm{~S}_{3} \ldots \ldots$

Argentite, $\mathrm{Ag}_{2} \mathrm{~S}$

Argyrodite, $4 \mathrm{Ag}_{2} \mathrm{~S}$. $\mathrm{GeS}_{2} \ldots \ldots \ldots \ldots$

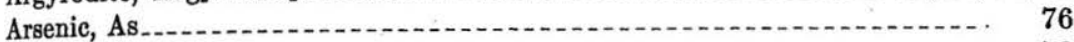

Arsenargentite, $\mathrm{Ag}_{3} \mathrm{As}$

Arsenoferrite, $\mathrm{FeAs}_{2}$

Arsenopyrite, FeAsS

Baumhauerite, $4 \mathrm{PbS} .3 \mathrm{As}_{2} \mathrm{~S}_{3} \ldots \ldots \ldots$

Benjaminite $(\mathrm{Cu}, \mathrm{Ag})_{2} \mathrm{~S} .2 \mathrm{PbS} .2 \mathrm{Bi}_{2} \mathrm{~S}_{3} \ldots \ldots \ldots$

Berthierite, FeS.Sb ${ }_{2} \mathrm{~S}_{3} \ldots$

Berthonite, $2\left(\mathrm{~Pb}, \mathrm{Cu}_{2}\right) \mathrm{S}_{2} \mathrm{Sb}_{2} \mathrm{~S}_{3} \ldots \ldots$

Bismuth, Bi........ 73

Bismuthinite, $\mathrm{Bi}_{2} \mathrm{~S}_{3} \ldots \ldots .78,78$

Bismutoplagionite, $5 \mathrm{PbS} .4 \mathrm{Bi}_{2} \mathrm{~S}_{3} \ldots \ldots \ldots$ 74, 82

Bornite, $\mathrm{Cu}_{5} \mathrm{FeS}_{4} \ldots$

Boulangerite, $5 \mathrm{PbS} .2 \mathrm{Sb}_{2} \mathrm{~S}_{3} \ldots \ldots \ldots \ldots .75,78$

Bournonite, $\mathrm{Cu}_{2} \mathrm{~S} .2 \mathrm{PbS} . \mathrm{Sb}_{2} \mathrm{~S}_{3} \ldots \ldots \ldots$. $\ldots 9,95$

Braunite, $3 \mathrm{Mn}_{2} \mathrm{O}_{3} \cdot \mathrm{MnSiO}_{2} \ldots \ldots \ldots$

Bravoite $(\mathrm{Fe}, \mathrm{Ni}) \mathrm{S}_{2} \ldots$

Breithauptite, NiSb_.......... 100

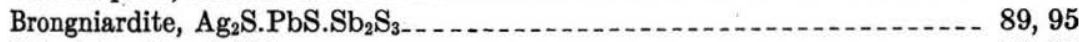

Calaverite ( $\mathrm{Au}, \mathrm{Ag}) \mathrm{Te}_{2} \ldots \ldots \ldots \ldots \ldots$ 76, 78

Canfieldite, $4 \mathrm{Ag}_{2} \mathrm{~S}_{\mathrm{SnS}} \mathrm{Sn}_{2}$ (contains some germanium) _... . . . . . . . . 83, 84

Carrollite $(\mathrm{Co}, \mathrm{Cu})_{3} \mathrm{~S}_{4}$

Cassiterite, $\mathrm{SnO}_{2} \ldots \ldots$

Cerargyrite, $\mathrm{AgCl}$

Chalcocite, $\mathrm{Cu}_{2} \mathrm{~S}_{2}$

Chalcophanite $(\mathrm{Mn}, \mathrm{Zn}) 0.2 \mathrm{MnO}_{2} .2 \mathrm{H}_{2} \mathrm{O} \ldots \ldots \ldots$

Chalcopyrite, $\mathrm{Cu}_{2} \mathrm{~S}_{\mathrm{Fe}} \mathrm{Fe}_{2} \mathrm{~S}_{3}$

Chalcostibite, $\mathrm{Cu}_{2} \mathrm{~S}_{2} \mathrm{Sb}_{2} \mathrm{~S}_{3}$

Chalmersite, CuS.2FeS (identical with cubanite) _.... 90, 95

*Chilenite, $\mathrm{Ag}_{6} \mathrm{Bi}$

*Chiviatite, $2 \mathrm{PbS} .3 \mathrm{Bi}_{2} \mathrm{~S}_{3}$

Chloanthite-smaltite $(\mathrm{Ni}, \mathrm{Co}) \mathrm{As}_{2}$

Chromite, $\mathrm{FeO} . \mathrm{Cr}_{2} \mathrm{O}_{3}$

Cinnabar, HgS.......... 95

Clausthalite, $\mathrm{PbSe}$

Cobaltite, CoAsS 
Coloradoite, HgTe.

*Comuccite. _.

Copper, Cu ........ 72, 7

Copper pitch ore (see Tenorite)

Corynite, $\mathrm{Ni}(\mathrm{As}, \mathrm{Sb}) \mathrm{S}$

Cosalite, 2PbS.Bi ${ }_{2} \mathrm{~S}_{3} \ldots \ldots . .74,81$

Covellite, CuS........ of

Cubanite, CuS.2FeS

Cuprite, $\mathrm{Cu}_{2} \mathrm{O} \ldots \ldots$

${ }^{*}$ Cuprobismutite, $3 \mathrm{Cu}_{2} \mathrm{~S} .4 \mathrm{Bi}_{2} \mathrm{~S}_{3}$

Cuprodescloizite, $\mathrm{Pb}_{2}(\mathrm{Zn}, \mathrm{Cu})_{2} \mathrm{~V}_{2} \mathrm{O}_{9} \cdot \mathrm{H}_{2} \mathrm{O} \ldots \ldots$.

Cylindrite, $6 \mathrm{PbS} . \mathrm{Sb}_{2} \mathrm{~S}_{3} .6 \mathrm{SnS}_{2} \ldots \ldots \ldots$

Delafossite, $\mathrm{CuFeO}_{2} \ldots \ldots$

*Dognacskaite, Cu,Bi,S

Domeykite, $\mathrm{Cu}_{3} \mathrm{As}$

Dufrenoysite, $2 \mathrm{PbS} . \mathrm{As}_{2} \mathrm{~S}_{3} \ldots \ldots \ldots \ldots$

Dyscrasite, $\mathrm{Ag}_{3} \mathrm{Sb}_{\ldots} \ldots \ldots \ldots$.

Electrum, $\mathrm{Ag}, \mathrm{Au}$

Emplectite, $\mathrm{Cu}_{2} \mathrm{~S} . \mathrm{Bi}_{2} \mathrm{~S}_{3} \ldots \ldots \ldots$.

Enargite, $\mathrm{Cu}_{2} \mathrm{~S} .4 \mathrm{CuS} . \mathrm{As}_{2} \mathrm{~S}_{3} \ldots \ldots \ldots \ldots$.

*Epiboulangerite, 3PbS.Sb${ }_{2} \mathrm{~S}_{5} \ldots \ldots$

Eucairite, $\mathrm{Cu}_{2} \mathrm{Se} . \mathrm{Ag}_{2} \mathrm{Se} \ldots \ldots \ldots$

Famatinite, $\mathrm{Cu}_{2} \mathrm{~S} .4 \mathrm{CuS} . \mathrm{Sb}_{2} \mathrm{~S}_{3} \ldots \ldots \ldots$

Ferberite, FeWO

Franckeite, $5 \mathrm{PbS} \mathrm{Sb}_{2} \mathrm{~S}_{3} \cdot 2 \mathrm{SnS}_{2} \ldots \ldots \ldots$.

Franklinite (Fe, $\mathrm{Zn}, \mathrm{Mn})$ O. $(\mathrm{Fe}, \mathrm{Mn})_{2} \mathrm{O}_{3} \ldots \ldots \ldots 1,102$

Freibergite, 5(Cu,Ag) ${ }_{2} \mathrm{~S} .2(\mathrm{Cu}, \mathrm{Fe}) \mathrm{S} .2 \mathrm{Sb}_{2} \mathrm{~S}_{3} \ldots \ldots \ldots$. . . . . . . . . . . . . .

Freieslebenite, $3 \mathrm{Ag}_{2} \mathrm{~S} .4 \mathrm{PbS} .3 \mathrm{Sb}_{2} \mathrm{~S}_{3} \ldots \ldots \ldots$ gl

Galena, PbS.

Galenobismutite, $\mathrm{PbS} . \mathrm{Bi}_{2} \mathrm{~S}_{3} \ldots$

Geoeronite, $5 \mathrm{PbS} . \mathrm{Sb}_{2} \mathrm{~S}_{3} \ldots \ldots \ldots$

Germanite, $\mathbf{C u}_{\mathbf{x}} \mathrm{GeS}_{\mathbf{y}} \ldots \ldots \ldots$ It

Gersdorffite, NiAsS

Glaueodot $(\mathrm{Co}, \mathrm{Fe}) \mathrm{AsS} \ldots \ldots$

Goethite, $\mathrm{Fe}_{2} \mathrm{O}_{3} \cdot \mathrm{H}_{2} \mathrm{O} \ldots$

Gold, $\mathrm{Au}$

Guanajuatite, $\mathrm{Bi}_{2}(\mathrm{Se}, \mathrm{S})_{3} \ldots \ldots$

*Guejarite, $\mathrm{Cu}_{2} \mathrm{~S} .2 \mathrm{Sb}_{2} \mathrm{~S}_{3} \ldots \ldots \ldots$

Guitermanite, $3 \mathrm{PbS} . \mathrm{As}_{2} \mathrm{~S}_{3} \ldots \ldots \ldots$ on

*Hauehecornite $(\mathrm{Ni}, \mathrm{Co})_{7}(\mathrm{~S}, \mathrm{Sb}, \mathrm{Bi})_{8} \ldots$

Hauerite, $\mathrm{MnS}_{2} \ldots \ldots \ldots \ldots$.

Hausmannite, $\mathrm{Mn}_{3} \mathrm{O}_{4} \ldots \ldots \ldots \ldots \ldots$. $22,92,99,101$

Hematite, $\mathrm{Fe}_{2} \mathrm{O}_{3} \ldots \ldots \ldots$. 102,109

*Hengleinite $(\mathrm{Fe}, \mathrm{Ni}, \mathrm{Co}) \mathrm{S}_{2} \ldots \ldots$

Hessite, $\mathrm{Ag}_{2} \mathrm{Te}_{\ldots} \ldots \ldots$

Hübnerite, $\mathrm{MnWO}_{4} \ldots$

Huntilite, $\mathrm{Ag}_{3} \mathrm{As}$ (identical with arsenargentite)

Ilmenite, $\mathrm{FeTiO}_{3}$

Jamesonite, 4PbS.FeS. $3 \mathrm{Sb}_{2} \mathrm{~S}_{3} \ldots \ldots \ldots$, 77

Jordanite, $4 \mathrm{PbS} . \mathrm{As}_{2} \mathrm{~S}_{3}$

*Kalgoorlite, $\mathrm{HgAu}_{2} \mathrm{Ag}_{6} \mathrm{Te}_{6}$

Kallilite, Ni(Sb,Bi)S

Keeleyite, $\mathrm{PbS} . \mathrm{Sb}_{2} \mathrm{~S}_{3}$

Kermesite, $\mathrm{Sb}_{2} \mathrm{~S}_{2} \mathrm{O}$ 
Klaprotholite (also known as klaprothite), $3 \mathrm{Cu}_{2} \mathrm{~S}_{2} 2 \mathrm{Bi}_{2} \mathrm{~S}_{3} \ldots \ldots \ldots \ldots, 85$

Klockmannite, CuSe....... 85

Krennerite; (Ag,Au) $\mathrm{Te}_{2}$

*Lehrbachite (PbSe+HgSe) -

Lengenbachite, $6 \mathrm{PbS} .\left(\mathrm{Ag}, \mathrm{Cu}{ }_{2} \mathrm{~S} .2 \mathrm{As}_{2} \mathrm{~S}_{3} \ldots \ldots\right.$

Leucopyrite, $\mathrm{Fe}_{3} \mathrm{As}_{4} \ldots$

LLimonite, $\mathrm{Fe}_{2} \mathrm{O}_{3} \cdot \mathrm{nH}_{2} \mathrm{O} \ldots \ldots$

Linnaeite, $\mathrm{Co}_{3} \mathrm{~S}_{4} \ldots$

Livingstonite, HgS.2 $\mathrm{Sb}_{2} \mathrm{~S}_{3} \ldots \ldots$

Löllingite, FeAs $\mathrm{F}_{2} \ldots 8$

Lorandite, $\mathrm{Tl}_{2} \mathrm{~S} . \mathrm{As}_{2} \mathrm{~S}_{3} \ldots$

Luzonite, $3 \mathrm{Cu}_{2} \mathrm{~S} . \mathrm{As}_{2} \mathrm{~S}_{5}$ (variety of enargite) $\ldots . . . . . .85,93$

Magnetite, $\mathrm{Fe}_{3} \mathrm{O}_{4} \ldots \ldots 103$

Manganite, $\mathrm{Mn}_{2} \mathrm{O}_{3} \ldots$

Marcasite, $\mathrm{FeS}_{2} \ldots 0$

Matildite, $\left(\mathrm{Ag}_{2}, \mathrm{~Pb}\right) \mathrm{S}_{\mathrm{B}} \mathrm{Bi}_{2} \mathrm{~S}_{3} \ldots \ldots$

Maucherite, $\mathrm{Ni}_{3} \mathrm{As}_{2} \ldots$

Melaconite, CuO (variety of tenorite) _... 82

Melonite, $\mathrm{Ni}_{2} \mathrm{Te}_{3}$

Meneghinite, $4 \mathrm{PbS} . \mathrm{Sb}_{2} \mathrm{~S}_{3} \ldots$

Metacinnabarite, $\mathrm{HgS}_{\ldots}$

Miargyrite, $\mathrm{Ag}_{2} \mathrm{~S} \mathrm{Sb}_{2} \mathrm{~S}_{3} \ldots \ldots \ldots, 84,93$

Millerite, NiS _..._. 89

Molybdenite, $\mathrm{MoS}_{2}$

Nagyagite, $\mathrm{Pb}, \mathrm{Au}, \mathrm{Te}, \mathrm{S}$

Naumannite, $\left(\mathrm{Ag}_{2}, \mathrm{~Pb}\right) \mathrm{Se}_{\ldots} \ldots$

Niccolite, NiAs.-. 98

*Onofrite, $\mathrm{Hg}(\mathrm{S}, \mathrm{Se})$.

Orpiment, $\mathrm{As}_{2} \mathrm{~S}_{3} \ldots$

Owyheeite, $8 \mathrm{PbS} .2 \mathrm{Ag}_{2} \mathrm{~S} .5 \mathrm{Sb}_{2} \mathrm{~S}_{3} \ldots$

Pearcite, $8 \mathrm{Ag}_{2} \mathrm{~S}_{\text {. }} \mathrm{As}_{2} \mathrm{~S}_{3} \ldots \ldots \ldots$

Pentlandite, (Ni,Fe)S............ 88

Petzite, $(\mathrm{Ag}, \mathrm{Au})_{2} \mathrm{Te} \ldots \ldots$.

Plagionite, $5 \mathrm{PbS} .4 \mathrm{Sb}_{2} \mathrm{~S}_{3} \ldots \ldots \ldots$

Plessite, $(\mathrm{Ni}, \mathrm{Fe})$ AsS

Polianite, $\mathrm{MnO}_{2}$

*Polyargyrite, $12 \mathrm{Ag}_{2} \mathrm{~S} \mathrm{Sb}_{2} \mathrm{~S}_{3} \ldots \ldots \ldots$

Polybasite, $8 \mathrm{Ag}_{2} \mathrm{~S}_{2} \mathrm{Sb}_{2} \mathrm{~S}_{3} \ldots \ldots \ldots$

Polydymite, $\mathrm{Ni}_{3} \mathrm{~S}_{4}$

Proustite, $3 \mathrm{Ag}_{2} \mathrm{~S}_{2} \mathrm{As}_{2} \mathrm{~S}_{3} \ldots \ldots \ldots$. 84

Psilomelane, $\mathrm{Mn}_{2} \mathrm{MnO}_{5}$

Pyrargyrite, $3 \mathrm{Ag}_{2} \mathrm{~S}_{2} \mathrm{Sb}_{2} \mathrm{~S}_{3} \ldots \ldots \ldots$ 8. 93

Pyrrhotite, $\mathrm{FeS}_{1+}+\ldots$

Pyrite, $\mathrm{FeS}_{2} \ldots$

Pyrolusite, $\mathrm{MnO}_{2}$

Rammelsbergite, $\mathrm{NiAs}_{2}$

Rathite, $3 \mathrm{PbS} .2 \mathrm{As}_{2} \mathrm{~S}_{3}$

Realgar, AsS

${ }^{*}$ Regnolite, $5(\mathrm{CuS}, \mathrm{FeS}, \mathrm{ZnS}) . \mathrm{As}_{2} \mathrm{~S}_{3}$

Rezbanyite, $2 \mathrm{PbS}_{3} 3 \mathrm{Bi}_{2} \mathrm{~S}_{3}$

Rickardite, $\mathrm{Cu}_{4} \mathrm{Te}_{3}(?)$

Rutile, $\mathrm{TiO}_{2} \ldots 3$

Schapbachite, $\mathrm{Ag}_{2} \mathrm{~S} . \mathrm{PbS}_{\mathrm{Bi}} \mathrm{Bi}_{2} \mathrm{~S}_{3}$ 
Seligmannite, $\mathrm{Cu}_{2} \mathrm{~S} .2 \mathrm{PbS} . \mathrm{As}_{2} \mathrm{~S}_{3}$

Semseyite, 9PbS.4Sb $\mathrm{Sb}_{2}$

Semeyite,

Siegenite, $(\mathrm{Co}, \mathrm{Ni})_{3} \mathrm{~S}_{4} \ldots \ldots$

Silver, Ag .

Skutterudite, (Co,Ni) $\mathrm{As}_{3} \ldots \ldots \ldots$ 97, 89

Smaltite-chloanthite $(\mathrm{Co}, \mathrm{Ni}) \mathrm{As}_{2} \ldots \ldots \ldots$ in

Sperrylite, $\mathrm{PtAs}_{2} \ldots \ldots$

Sphalerite, ZnS. . .

Stannite, $\mathrm{Cu}_{2}$ S.FeS.SnS $2 \ldots \ldots \ldots$........

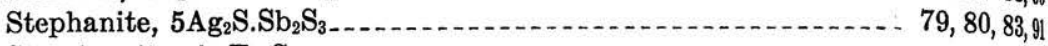

Sternbergite, $\mathrm{AgFe} \mathrm{S}_{3}$

Stibnite, $\mathrm{Sb}_{2} \mathrm{~S}_{3} \ldots \ldots \ldots \ldots \ldots .73,80,85$

Stromeyerite, $(\mathrm{Cu}, \mathrm{Ag})_{2} \mathrm{~S} \ldots \ldots$

Sulvanite, $\mathrm{Cu}_{3} \mathrm{VS}_{4} \ldots$

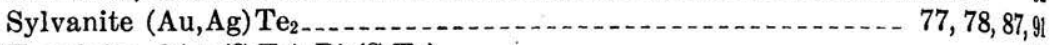

*Tapalpite, $3 \mathrm{Ag}_{2}(\mathrm{~S}, \mathrm{Te}) \cdot \mathrm{Bi}_{2}(\mathrm{~S}, \mathrm{Te})_{3} \ldots \ldots \ldots$

Teallite, SnS.PbS . _ .

Tellurium, Te_.

Tennantite, $5 \mathrm{Cu}_{2} \mathrm{~S} .2(\mathrm{Cu}, \mathrm{Fe}, \mathrm{Zn}) \mathrm{S} .2 \mathrm{As}_{2} \mathrm{~S}_{3} \ldots \ldots \ldots . . . .84$

Tenorite, $\mathrm{CuO} \ldots$

Tetradymite, $\mathrm{Bi}_{2}(\mathrm{Te}, \mathrm{S})_{3} \ldots \ldots \ldots$.

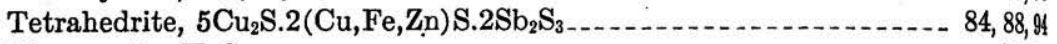

Tiemannite, HgSe_...

Troilite, FeS........ 74

Tungstenite, $\mathrm{WS}_{2} \ldots \ldots$

*Turgite, $2 \mathrm{Fe}_{2} \mathrm{O}_{3} \cdot \mathrm{H}_{2} \mathrm{O} \ldots \ldots \ldots \ldots$.

Ullmanite, NiSbS. . . . .

Umangite, $\mathrm{Cu}_{3} \mathrm{Se}_{2} \ldots \ldots \ldots \ldots$

Uraninite, uranate of $\mathrm{U}, \mathrm{Pb}$, etc ............. 102

Violarite, $(\mathrm{Ni}, \mathrm{Fe})_{3} \mathrm{~S}_{4} \ldots \ldots \ldots \ldots$

Voltzite, $4 \mathrm{ZnS} . \mathrm{ZnO} \ldots$

Vrbaite, $\mathrm{Tl}_{2} \mathrm{~S} .3(\mathrm{As}, \mathrm{Sb})_{2} \mathrm{~S}_{3 \ldots \ldots} \ldots \ldots \ldots \ldots$

Weissite, $\mathrm{Cu}_{5} \mathrm{Te}_{3}($ ? $\ldots \ldots \ldots$

Whitneyite, $\mathrm{Cu}_{9} \mathrm{As}$

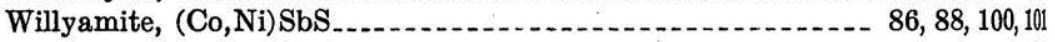

Wittichenite, $3 \mathrm{Cu}_{2} \mathrm{~S} \mathrm{Bi}_{2} \mathrm{~S}_{3} \ldots \ldots \ldots \ldots$

Wolframite, (Fe, Mn) WO

Wurtzite, ZnS.

Zinkenite, $\mathrm{PbS} \mathrm{Sb}_{2} \mathrm{~S}_{3} \ldots \ldots$ 
OUTLINE OF TABLE

Soft minerals

\begin{tabular}{|c|c|c|c|c|c|c|c|c|c|}
\hline & \multirow{2}{*}{\multicolumn{2}{|c|}{ Reagents }} & & \multicolumn{3}{|c|}{ Isotropic } & \multicolumn{3}{|c|}{ Anisotropic } \\
\hline & & & & Mineral & Formula & Page & Mineral & Formula & Page \\
\hline \multirow[t]{6}{*}{$\underset{\text { eff. }}{\mathrm{HNO}_{3}}$} & \multirow[t]{4}{*}{$\mathrm{HCl}$} & \multirow[t]{2}{*}{$\mathrm{KCN}$} & $\mathrm{FeCl}_{3}$ & $\begin{array}{l}\text { Copper arsenides: } \\
\text { Domeykite. } \\
\text { Algodonite- } \\
\text { Whitneyite_. } \\
\text { Copper (native) } \\
\text { Arsenargentite } \\
\text { Chalcocite } \\
\text { Silver (native) }\end{array}$ & 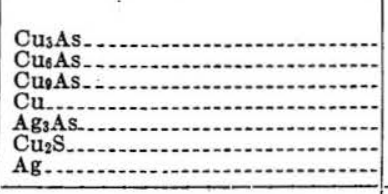 & \begin{tabular}{l|}
71 \\
71 \\
71 \\
72 \\
72 \\
72 \\
72
\end{tabular} & $\begin{array}{l}\text { Rickardite._. } \\
\text { Weissite } \\
\text { Chalocite... } \\
\text { Cuprite........... }\end{array}$ & $\begin{array}{l}\mathrm{Cu}_{4} \mathrm{Te}_{3}(?) \\
\mathrm{Cu}_{5} \mathrm{Te}_{3}(?) \\
\mathrm{Cu}_{2} \mathrm{~S}_{2} \\
\mathrm{Cu}_{2} \mathrm{O}\end{array}$ & $\begin{array}{l}72 \\
72 \\
72 \\
73\end{array}$ \\
\hline & & & $\begin{array}{c}\mathrm{FeCl}_{3} \\
\text { neg. }\end{array}$ & & & $\cdots$ & Stibnite............. & $\mathrm{Sb}_{2} \mathrm{~S}_{3} \ldots$ & 73 \\
\hline & & \multirow[t]{2}{*}{$\begin{array}{r}\mathrm{KCN} \\
\text { neg. }\end{array}$} & $\mathrm{FeCl}_{3}$ & $\begin{array}{l}\text { Silver } \\
\text { Altaite_. } \\
\text { Galena... }\end{array}$ & $\begin{array}{l}\mathrm{Ag} \\
\mathrm{PbTe} \\
\mathrm{PbS}\end{array}$ & \begin{tabular}{l|l}
73 \\
73 \\
73
\end{tabular} & $\begin{array}{l}\text { Bismuth } \\
\text { Tetradymite. } \\
\text { Matildite.... } \\
\text { Tellurium.... } \\
\text { Cosalite...... }\end{array}$ & $\begin{array}{l}\mathrm{Bi} \\
2 \mathrm{Bi}_{2} \mathrm{Te}_{3} \mathrm{Bi}_{2} \mathrm{~S}_{3} \\
\left(\mathrm{Ag}_{2} \mathrm{~Pb}\right) \mathrm{S}_{3} \mathrm{Bi}_{2} \mathrm{~S}_{3} \\
\mathrm{Te}_{2} \\
2 \mathrm{PbS} . \mathrm{Bi}_{2} \mathrm{~S}_{3}\end{array}$ & $\begin{array}{l}73 \\
73 \\
73 \\
74 \\
74 \\
\end{array}$ \\
\hline & & & $\begin{array}{r}\mathrm{FeCl}_{3} \\
\text { neg. }\end{array}$ & Alabandite........ & MnS....................................... & 74 & $\begin{array}{l}\text { Jamesonite } \\
\text { Plagionite } \\
\text { Semseyite } \\
\text { Zinkenite } \\
\text { Troilite } \\
\text { Bismuthinite } \\
\text { Bismutoplagionite } \\
\text { Boulangerite. } \\
\text { Meneghinite... }\end{array}$ & 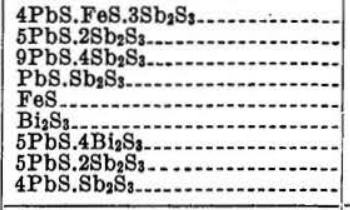 & $\begin{array}{l}74 \\
74 \\
74 \\
74 \\
74 \\
74 \\
74 \\
75 \\
75 \\
\end{array}$ \\
\hline & \multirow[t]{2}{*}{$\begin{array}{l}\text { HCl } \\
\text { neg. }\end{array}$} & \multirow[t]{2}{*}{$\mathrm{KCN}$} & $\mathrm{FeCl}_{3}$ & 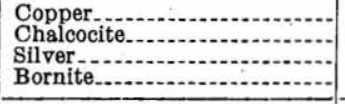 & $\begin{array}{l}\mathrm{Cu} \\
\mathrm{Cu}{ }_{2} \mathrm{~S}_{1} \\
\mathrm{Ag} \mathrm{Cu}_{5} \mathrm{FeS} \mathrm{S}_{4}\end{array}$ & $\begin{array}{l}75 \\
75 \\
75 \\
75 \\
\end{array}$ & $\begin{array}{l}\text { Chalcocite } \\
\text { Bornite.... }\end{array}$ & $\begin{array}{l}\mathrm{Cu}_{2} \mathrm{~S} \\
\mathrm{Cu} \mathrm{FeS}_{4}\end{array}$ & $\begin{array}{l}75 \\
76\end{array}$ \\
\hline & & & $\begin{array}{r}\mathrm{FeCl}_{3} \\
\text { neg. }\end{array}$ & Electrum.............................. & $\mathrm{Au}, \mathrm{Ag}$ & 76 & Klaprotholite......... & $3 \mathrm{Cu}_{2} \mathrm{~S} .2 \mathrm{Bi}_{2} \mathrm{~S}_{3} \ldots \ldots$ & 76 \\
\hline
\end{tabular}




\begin{tabular}{|c|c|c|c|c|c|c|c|c|c|}
\hline & & & & \multicolumn{3}{|c|}{ Isotropic } & \multicolumn{3}{|c|}{ Anisotropic } \\
\hline \multicolumn{4}{|c|}{ Reagents } & Mineral & Formula & Page & Mineral & Formula & Page \\
\hline \multirow[t]{2}{*}{$\begin{array}{l}\mathrm{HNO}_{\text {elf. }} \\
\mathrm{HNO}_{3}\end{array}$} & \multirow[t]{2}{*}{$\begin{array}{l}\mathrm{HCl} \\
\text { neg. }\end{array}$} & \multirow[t]{2}{*}{$\begin{array}{r}\mathrm{KCN} \\
\text { neg. }\end{array}$} & $\mathrm{FeCl}_{3}$ & $\cdots$ & & & 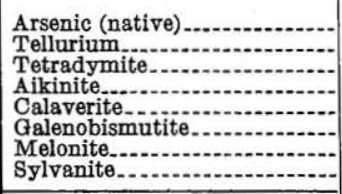 & $\begin{array}{l}\mathrm{As} \\
\mathrm{Te} \\
\mathrm{Bi}_{2}\left(\mathrm{Te}, \mathrm{S}_{3}\right)_{3} \\
\mathrm{Cu}_{2} \mathrm{~S} .2 \mathrm{PbS} . \mathrm{Bi}_{2} \mathrm{~S}_{3} \\
(\mathrm{Au}, \mathrm{Ag}) \mathrm{Te}_{2} \\
\mathrm{PbS} \mathrm{Bi}_{2} \mathrm{~S}_{3} \\
\mathrm{Ni}_{2} \mathrm{Te}_{3}-\mathrm{Pe}_{2} \\
(\mathrm{Ag}, \mathrm{Au}) \mathrm{Te}_{2}\end{array}$ & $\begin{array}{l}76 \\
76 \\
76 \\
76 \\
76 \\
77 \\
77 \\
77 \\
\end{array}$ \\
\hline & & & $\begin{array}{c}\mathrm{FeCl}_{3} \\
\text { neg. }\end{array}$ & $\begin{array}{l}\text { Poldymite } \\
\text { Sphalerite. } \\
\text { Violarite- }\end{array}$ & $\begin{array}{l}\mathrm{Ni}_{3} \mathrm{~S}_{4} \\
\mathrm{ZnS} \\
(\mathrm{Ni}, \mathrm{Fe})_{3} \mathrm{~S}_{4}\end{array}$ & $\begin{array}{l}77 \\
77 \\
77\end{array}$ & 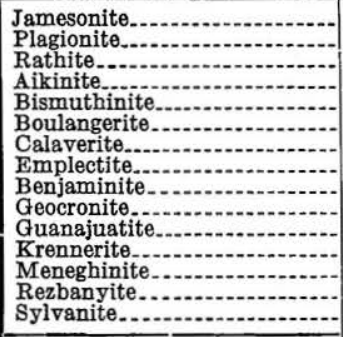 & 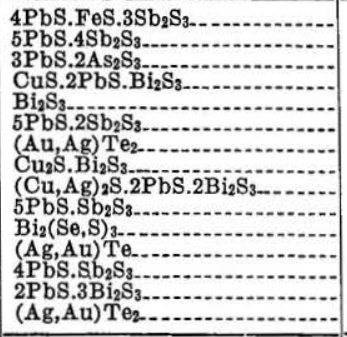 & $\begin{array}{l}77 \\
77 \\
78 \\
78 \\
78 \\
78 \\
78 \\
78 \\
78 \\
78 \\
78 \\
78 \\
78 \\
78 \\
78 \\
\end{array}$ \\
\hline \multirow[t]{3}{*}{$\mathrm{HNO}_{3}$} & \multirow[t]{3}{*}{$\mathrm{HCl}$} & \multirow[t]{2}{*}{$\mathrm{KCN}$} & $\mathrm{FeCl}_{3}$ & 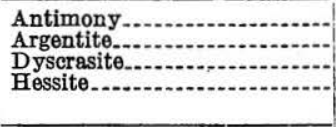 & $\begin{array}{l}\mathrm{Sb} \\
\mathrm{Ag}_{2} \mathrm{~S}_{\mathrm{B}} \\
\mathrm{Ag}_{3} \mathrm{Sb}- \\
\mathrm{Ag}_{2} \mathrm{~T} \mathrm{e}\end{array}$ & $\begin{array}{l}79 \\
79 \\
79 \\
79\end{array}$ & 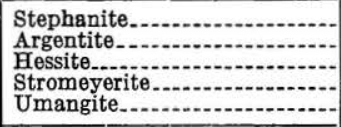 & $\begin{array}{l}5 \mathrm{Ag}_{2} \mathrm{~S} . \mathrm{Sb}_{2} \mathrm{~S}_{3} \\
\mathrm{Ag}_{2} \mathrm{~S} \\
\mathrm{Ag}_{2} \mathrm{Te} \\
(\mathrm{Cu}, \mathrm{Ag})_{2} \mathrm{~S}_{2} \\
\mathrm{Cu}_{3} \mathrm{Se}_{2}\end{array}$ & $\begin{array}{l}79 \\
79 \\
79 \\
79 \\
79 \\
\end{array}$ \\
\hline & & & $\begin{array}{r}\mathrm{FeCl}_{3} \\
\text { neg. }\end{array}$ & & & & $\begin{array}{l}\text { Stephanite. } \\
\text { Stibnite. }\end{array}$ & $\begin{array}{l}5 \mathrm{Ag}_{2} \mathrm{~S} . \mathrm{Sb}_{2} \mathrm{~S}_{3} \\
\mathrm{Sb}_{2} \mathrm{~S}_{3}\end{array}$ & $\begin{array}{l}80 \\
80\end{array}$ \\
\hline & & $\underset{\text { neg. }}{\mathrm{KCN}}$ & $\mathrm{FeCl}_{3}$ & $\begin{array}{l}\text { Aguilarite } \\
\text { Petzite } \\
\text { Olausthalite- } \\
\text { Galena. }\end{array}$ & $\begin{array}{l}\mathrm{Ag}_{2}(\mathrm{Se}, \mathrm{S}) \\
(\mathrm{Ag}, \mathrm{Au})_{2} \mathrm{Te}_{2} \\
\mathrm{PbS} \mathrm{PbS}_{-}\end{array}$ & $\begin{array}{l}80 \\
80 \\
80 \\
80\end{array}$ & 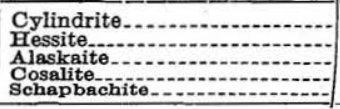 & 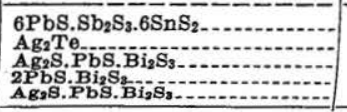 & $\begin{array}{l}80 \\
81 \\
81 \\
81 \\
81\end{array}$ \\
\hline
\end{tabular}



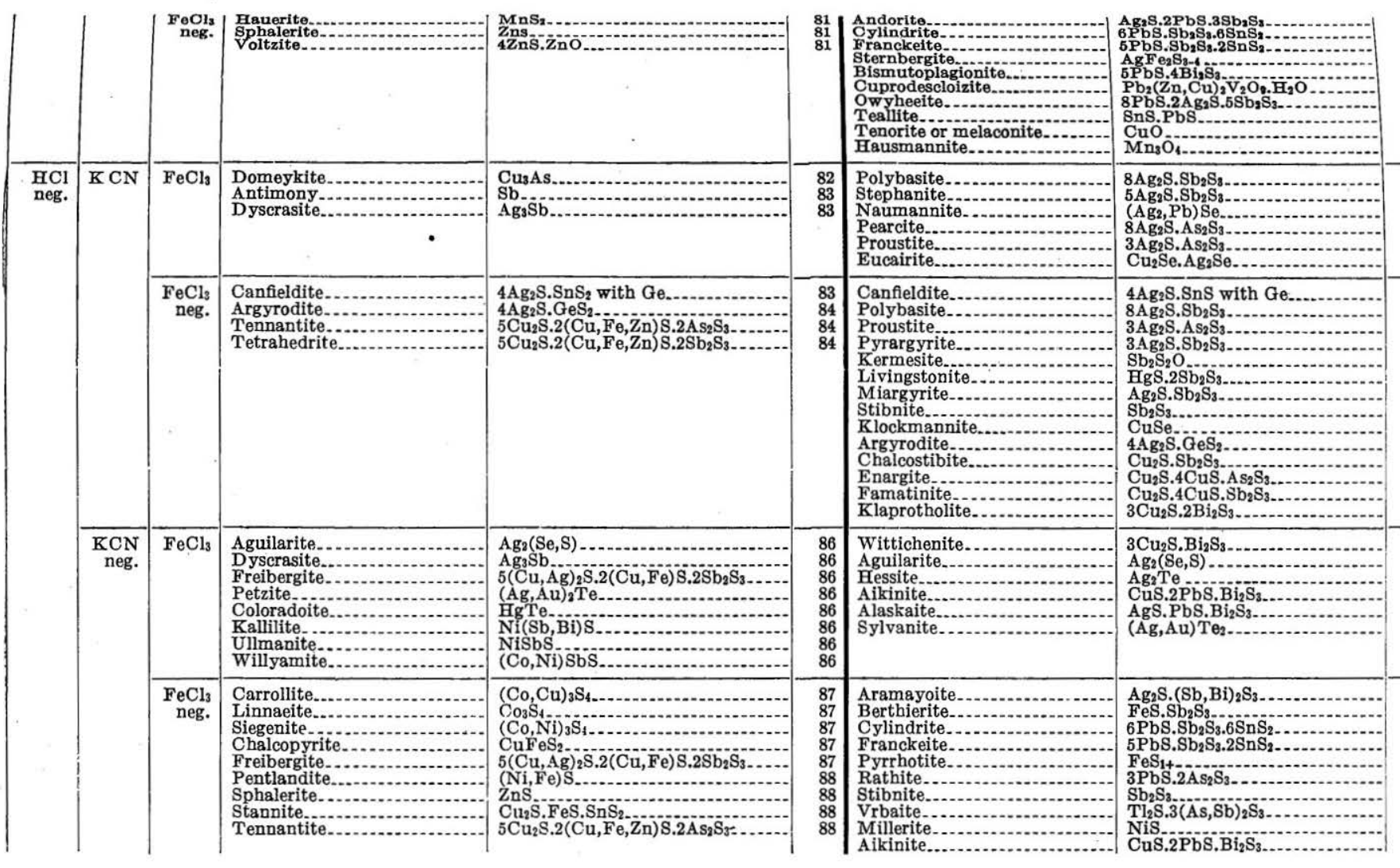


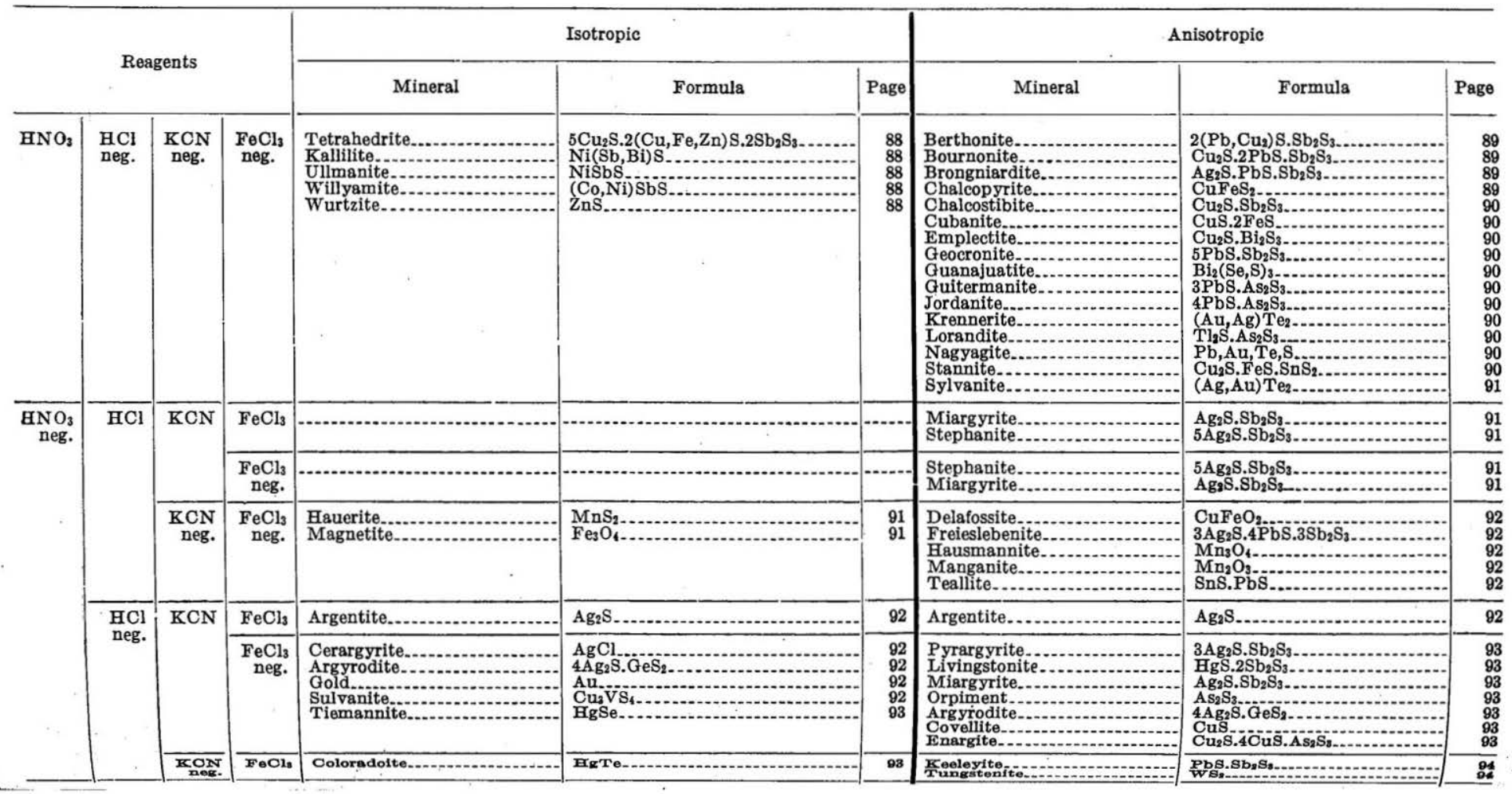



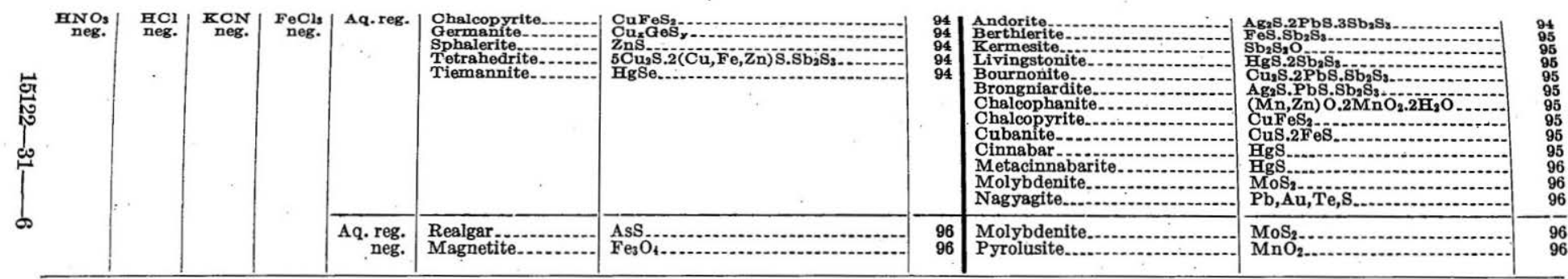

Hard minerals

\begin{tabular}{|c|c|c|c|c|c|c|c|c|c|}
\hline & & & & & Isotropic & & & Inisotropio & \\
\hline & & & & Mineral & Formula & Page & Mineral & Formula & Page \\
\hline$\underset{\text { eff. }}{\mathrm{HNO}_{3}}$ & $\begin{array}{l}\text { KCl } \\
\text { neg. }\end{array}$ & $\begin{array}{l}\text { KCN } \\
\text { neg. }\end{array}$ & $\mathrm{FeCl}_{3}$ & $\begin{array}{l}\text { Chloanthite-smaltite.. } \\
\text { Corynite } \\
\text { Maucherite } \\
\text { Smaltite-chloanthite. } \\
\text { Arsenoferrite } \\
\text { Gersdorffite } \\
\text { Skutterudite_... }\end{array}$ & $\begin{array}{l}(\mathrm{Ni}, \mathrm{Co}) \mathrm{As}_{2} \\
\mathrm{Ni}(\mathrm{As}, \mathrm{Sb}) \mathrm{S} \\
\mathrm{Ni} \mathrm{S}_{2} \mathrm{As}_{2} \\
(\mathrm{Co}, \mathrm{Ni}) \mathrm{As}_{2} \\
\mathrm{FeAs} 2 \\
\mathrm{NiAs} \\
(\mathrm{Co}, \mathrm{Ni}) \mathrm{As}_{3}\end{array}$ & $\begin{array}{l}97 \\
97 \\
97 \\
97 \\
97 \\
97 \\
97 \\
\end{array}$ & $\begin{array}{l}\text { Maucherite } \\
\text { Niccolite } \\
\text { Plessite } \\
\text { Rammgisbergite............ }\end{array}$ & $\begin{array}{l}\mathrm{Ni}_{3} \mathrm{As} \\
\mathrm{NiAs_{2 }} \\
\text { (Ni,Fe) AsS } \\
\text { NiAs2 }\end{array}$ & $\begin{array}{l}98 \\
98 \\
98 \\
98\end{array}$ \\
\hline & & . & $\begin{array}{r}\mathrm{FeCl}_{3} \\
\text { neg. }\end{array}$ & $\begin{array}{l}\text { Gersdorffite } \\
\text { Pyrite. }\end{array}$ & $\begin{array}{l}\text { NiAsS } \\
\mathrm{FeS}_{2}\end{array}$ & $\begin{array}{l}98 \\
98\end{array}$ & $\begin{array}{l}\text { Arsenopyrite } \\
\text { Löllingite (nickeliferous) } \\
\text { Marcasite... } \\
\text { Pyrite... }\end{array}$ & $\begin{array}{l}\mathrm{FeAsS} \\
(\mathrm{Fe}, \mathrm{Ni}) \mathrm{As}_{2} \\
\mathrm{FeS}_{2} \\
\mathrm{FeS}_{2}\end{array}$ & $\begin{array}{l}98 \\
98 \\
98 \\
99 \\
\end{array}$ \\
\hline $\mathrm{HNO}_{3}$ & $\mathrm{HCl}$ & $\mathrm{KCN}$ & $\mathrm{FeCl}_{3}$ & Psilomelane ....................... & $\mathrm{Mn}_{2} \mathrm{MnO}_{3}$ & 99 & & & \\
\hline & & & $\begin{array}{l}\mathrm{FeCl}_{3} \\
\text { neg. }\end{array}$ & - & & 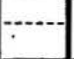 & Hausmannite...... & $\mathrm{Mn}_{3} \mathrm{O}_{4}$ & 99 \\
\hline & $\begin{array}{l}\mathrm{HCl} \\
\text { neg. }\end{array}$ & $\underset{\text { neg. }}{\text { KCN }}$ & $\mathrm{FeCl}_{3}$ & $\begin{array}{l}\text { Skutterudite (ferriferous) } \\
\text { Kallilite.... } \\
\text { Ullmanite. } \\
\text { Willyamite...-. }\end{array}$ & $\begin{array}{l}(\mathrm{Co}, \mathrm{Ni}, \mathrm{Fe}) \mathrm{As}_{3} \\
\mathrm{Ni}(\mathrm{Sb}, \mathrm{Bi}) \mathrm{S} \\
\mathrm{NiSbS} \\
(\mathrm{Co}, \mathrm{Ni}) \mathrm{SbS} \mathrm{S} .\end{array}$ & $\begin{array}{r}99 \\
99 \\
100 \\
100 \\
\end{array}$ & Breithauptite ................... & 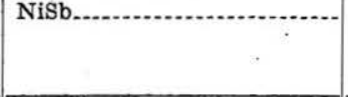 & 100 \\
\hline
\end{tabular}




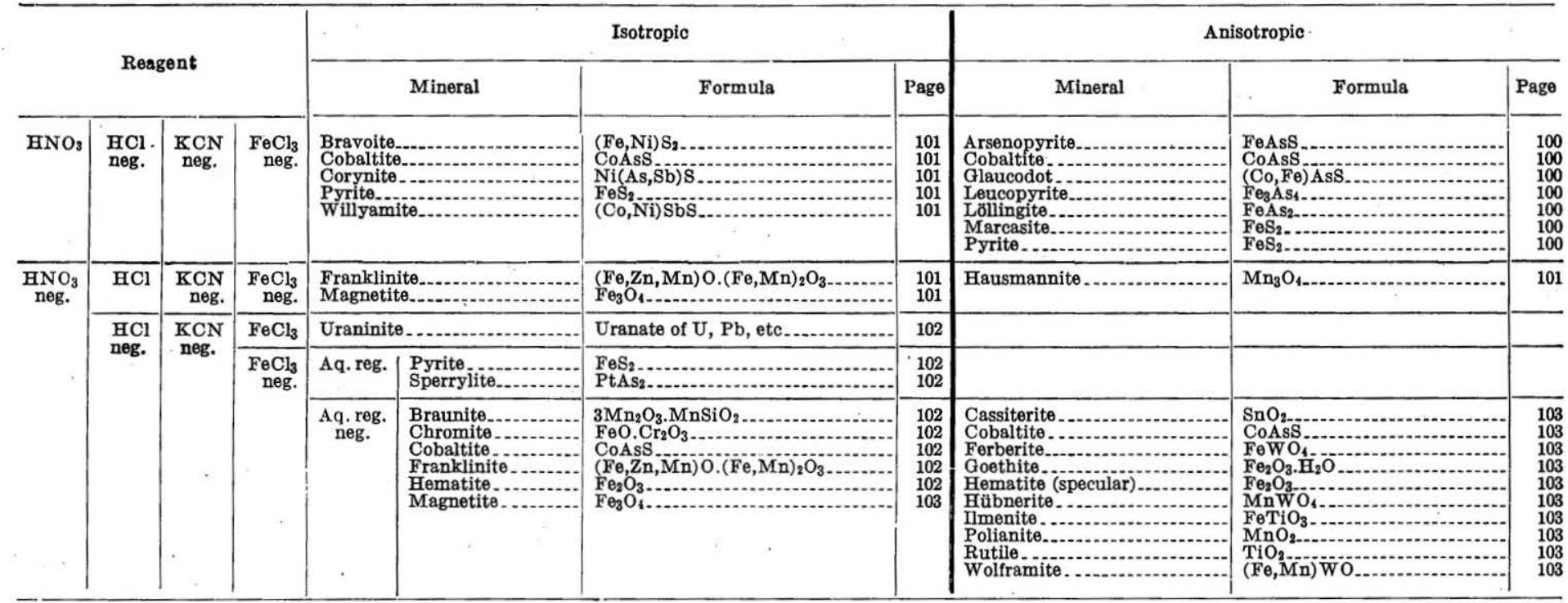




\title{
SOFT MINERALS
}

\author{
$\mathrm{HNO}_{3}$ eff., $\mathrm{HCl}, \mathrm{KCN}, \mathrm{FeCl}_{3}$
}

\author{
ISOTROPIC
}

Copper arsenides.--Textbooks in mineralogy usually describe three arsenides of copper-domeykite $\left(\mathrm{Cu}_{3} \mathrm{As}\right)$, algodonite $\left(\mathrm{Cu}_{6} \mathrm{As}\right)$, and whitneyite $\left(\mathrm{Cu}_{0} \mathrm{As}\right)$. Recent microscopic investigation has shown that algodonite is really an intergrowth of two distinct minerals which are closely similar in color and etch tests. In fact, the two constituents are rarely recognized under the microscope before etching, but they are brought out by reagents owing to the fact that one constituent etches more rapidly than the other. Similarly "whitneyite" comprises four distinct minerals, at least two of which are usually present in any specimen. It is not considered feasible for the average investigator to separate the individual constituents of algodonite or whitneyite; this requires considerable experience with these ores. It is possible, however, to distinguish between the different groups by following the descriptions given below. More complete details are given by Broderick. ${ }^{43}$

Domeykite $\left(\mathrm{Cu}_{3} \mathrm{As}\right)$.- Isometric; color white; hardness $\mathrm{C}-$. KоH $\mathrm{HNO}_{3}$ effervesces, turns dark gray, and brings out a triangular $\mathrm{HgCl}_{2}$ etch cleavage. $\mathrm{HCl}$ brings out a triangular etch pattern; mineral stains slowly. KCN slowly brings out triangular etch pattern; surface does not stain. $\mathrm{FeCl}_{3}$ instantly stains black. $\mathrm{KOH}$ stains differentially iridescent and brings out triangular etch pattern. $\mathrm{HgCl}_{2}$ tarnishes black. Domeykite is an authentic mineral, readily distinguished from algodonite and whitneyite by its color. In oblique reflected light, color has a suggestion of yellow. Fairly sectile but more brittle than other arsenides of copper.

Algodonite $\left(\mathrm{Cu}_{8} \mathrm{As}\right)$.- -Isometric; color greenish gray; hardness $\mathrm{C}-$. $\mathrm{HNO}_{3}$ effervesces and blackens; some varieties give parallel (possibly cubic) etch cleavage. $\mathrm{HCl}$ slowly stains brown. KCN stains brown; some varieties show parallel etch cleavage. $\mathrm{FeCl}_{3}$ instantly stains black. $\mathrm{KOH}$ slowly stains iridescent. $\mathrm{HgCl}_{2}$ instantly stains black. Algodonite is an intergrowth of two constituents, each a definite mineral, termed $\alpha$ and $\beta$ algodonite. $\alpha$-algodonite is greenish gray, $\beta$-algodonite has a suggestion of blue. With $\mathrm{HCl} \alpha$-algodonite etches more rapidly than $\beta$-algodonite. More sectile than domeykite.

Whitneyite ( $\left.\mathrm{Cu}_{9} \mathrm{As}\right)$.-Isometric; color pale coppery pink; hardness $\mathrm{C}-$. $\mathrm{HNO}_{3}$ effervesces vigorously and stains iridescent; on some varieties brings out grain structure; with others etching brings out a distinct lamellar structure. $\mathrm{HCl}$ slowly stains gray. KCN slowly browns; with some varieties reagent effervesces slowly. $\mathrm{FeCl}_{3}$ instantly blackens. $\mathrm{KOH}$ slowly stains iridescent. $\mathrm{HgCl}_{2}$ instantly stains brown to black. Four distinct constituents bave been recognized in whitneyite. These are termed $\alpha, \beta, \gamma$, and $\delta$ whitneyite. The first three are a distinctly lighter shade of pink than native copper, but the last is almost indistinguishable from copper in color. They vary in color from $\alpha$, the lightest, to $\delta$, the most distinctly coppery. Some of these constituents may be alloys of copper and arsenic rather than definite chemical compounds. Whitneyite is more brittle than native copper. In polishing, pits form on whitneyite, whereas they are almost completely absent on copper. On the other hand, polishing scratches are more numerous and deeper on copper than on whitneyite. A good method of distinguishing between copper and whitneyite is to apply a drop of 1: $5 \mathrm{HCl}$. Whitneyite etches readily, whereas copper is negative. The best test for whitneyite, however, is a microchemical test for arsenic.

\footnotetext{
"Broderick, T. M., in Butler, B. S., and others, The copper deposits of A Michigan: U. S. Geol. Survey $_{\text {Prof. Paper 144, p. 56, 1929. }}$
} 
Copper $(\mathrm{Cu})$.- - Isometric; color coppery red; hardness $\mathrm{B}+. \quad \mathrm{HNO}_{3}$ efferveseces; some varieties tarnish brown; others seem to effervesce without tarnishing. $\mathrm{HCl}$ negative; some varieties slowly tarnish brown. KCN slowly browns. $\mathrm{FeCl}_{3}$ quickly darkens. $\mathrm{KOH}$ slowly tarnishes brown to iridescent. $\mathrm{HgO}$ quickly blackens. Sectile.

Arsenargentite $\left(\mathrm{Ag}_{3} \mathrm{As}\right)$.- Isometric; color creamy white; hardness $\mathrm{B}$. $\mathrm{HNO}$ effervesces and stains black. $\mathrm{HCl}$ tarnishes iridescent. KCN tarnishes brom, $\mathrm{FeCl}_{3}$ instantly tarnishes iridescent. $\mathrm{KOH}$ stains brown. $\mathrm{HgCl}_{2}$ tarnishee brown. Almost sectile. Very rare. Identical with huntilite.

Chalcocite $\left(\mathrm{Cu}_{2} \mathrm{~S}\right)$. - Isometric and orthorhombic; color bluish KOH neg. gray; hardness B. Hypogene chalcocite with more than 8 per $\mathrm{HgCl}_{2}$ cent of dissolved covellite is isotropic. Very fine grained 80 . pergene chalcocite is also apparently isotropic. Most chalcocite, both hypogene and supergene, is weakly anisotropic. $\mathrm{HNO}_{3}$ effervesces vigor. ously; mineral stains blue; with some isotropic varieties brings out octahedral etch cleavage; very fine grained supergene chalcocite shows no etch cleavage. $\mathrm{HCl}$ negative; some varieties tarnish faintly. KCN quickly stains black; first brings out etch cleavage, but if solution is allowed to remain on surface, the etch cleavage is destroyed, and a rough, corroded surface remains. $\mathrm{FeCl}_{3}$ stains blue; some isotropic varieties show octahedral etch cleavage; very fine grained super. gene chalcocite, however, does not show etch cleavage. $\mathrm{KOH}$ negative. $\mathrm{HgCl}$ tarnishes slightly.

Silver (Ag).-Isometric; color silvery white; hardness $\mathrm{B} . \mathrm{HNO}_{3}$ effervescer slightly for a moment; stains gray. $\mathrm{HCl}$ fumes tarnish slightly; almost negative $\mathrm{KCN}$ tarnishes dark gray to brown. $\mathrm{FeCl}_{3}$ instantly stains iridescent. $\mathrm{KOH}$ negative. $\mathrm{HgCl}_{2}$ stains brown to iridescent. Very sectile.

\section{ANISOTROPIC}

Rickardite $\left(\mathrm{Cu}_{4} \mathrm{Te}_{3}\right.$ ?).-Crystal system unknown; color purplish KOH red, similar to bornite; hardness B. Strong anisotropism; gives $\mathrm{HgCl}_{2}$ four extinctions per revolution. Interference color fiery orange, very similar to that of umangite. $\mathrm{HNO}_{3}$ blackens with effer. vescence. $\mathrm{HCl}$ etches gray to brown. $\mathrm{KCN}$ slowly bleaches gray. $\mathrm{FeO}_{4}$ bleaches gray to brown. $\mathrm{KOH}$ tarnishes iridescent to black. $\mathrm{HgCl}_{2}$ slowly tarnishes purple. Very rare; reported only from Vulcan, Colo. Always inter. grown with weissite and other tellurides, hence formula given in textbooks is probably incorrect.

Weissite $\left(\mathrm{Cu}_{5} \mathrm{Te}_{3}\right.$ ?).-Crystal system unknown; color light gray, about the same as chalcocite; hardness $\mathrm{B}$. $\mathrm{HNO}_{3}$ effervesces and stains brown. $\mathrm{HO}$ stains light brown. $\mathrm{KCN}$ stains light brown. $\mathrm{FeCl}_{3}$ stains brown. $\mathrm{KOH}$ slowly stains brown. $\mathrm{HgCl}_{2}$ stains light brown. Specimen from Vulcan, Colan Always associated with rickardite $\left(\mathrm{Cu}_{4} \mathrm{Te}_{3}\right)$. Distinguished from rickardite by color.

KOH neg. $\quad$ Chalcocite $\left(\mathrm{Cu}_{2} \mathrm{~S}\right)$. - Isometric and orthorhombic; color bluish $\begin{array}{ll}\text { KOH neg. } & \text { gray; hardness B. Most chalcocite is weakly anisotropic. HNo; } \\ \mathbf{H g C l}_{2} & \text { effervesces vigorously; minera! stains blue; with most varietien }\end{array}$ brings out parallel etch structure; very fine grained supergene chalcocite, however, will not show etch cleavage; with fine-grained sooty chalovcite brings out grain boundaries only. $\mathrm{HCl}$ negative; some varieties tarnish faintly. KCN quickly stains black; first brings out etch cleavage, but if drop is allowed to remain etch cleavage is destroyed, and a rough, corroded surface remains. $\mathrm{FeCl}_{3}$ stains blue; with most specimens brings out parallel etch clesr. age. $\mathrm{KOH}$ negative. $\mathrm{HgCl}_{2}$ tarnishes slightly. 
Cuprite $\left(\mathrm{Cu}_{2} \mathrm{O}\right)$.-Isometric; color bluish gray; hardness $\mathrm{D}$ - . коH neg. $\mathrm{HNO}_{3}$ effervesces and deposits coating of metallic copper. $\mathrm{HgCl}_{2}$ neg. $\mathrm{HCl}$ deposits white coating. $\mathrm{KCN}$ darkens and brings out parallel etch structure. $\mathrm{FeCl}_{3}$ tarnishes iridescent. $\mathrm{KOH}$ and $\mathrm{HgCl}_{2}$ negative. Ruby-red powder and internal reflection.

\section{$\mathrm{HNO}_{3}$ eff., $\mathrm{HCl}, \mathrm{KCN}, \mathrm{FeCl}_{3}$ neg.}

\section{ANISOTROPIC}

Stibnite $\left(\mathrm{Sb}_{2} \mathrm{~S}_{3}\right)$.-Orthorhombic; color gray; hardness $\mathrm{B}$.

KOH

$\mathrm{HgCl}_{2}$ neg.

Very strong anisotropism. $\mathrm{HNO}_{3}$ stains differentially iridescent; some varieties effervesce slowly. $\mathrm{HCl}$ fumes tarnish; some varieties negative. KCN brings out scratches and sometimes stains slightly. $\mathrm{FeCl}_{3}$ negative. $\mathrm{KOH}$ instantly tarnishes brown and gives yellow coating; this is the most characteristic reaction for stibnite. $\mathrm{HgCl}_{2}$ negative. Prismatic. Hardness and color vary somewhat with orientation, giving mottled appearance suggesting the presence of more than one mineral.

\section{$\mathrm{HNO}_{3}$ eff., $\mathrm{HCl}, \mathrm{KCN}$ neg., $\mathrm{FeCl}_{3}$}

ISOTROPIC

Silver (Ag).--Isometric; color silvery white; hardness B. KоH neg. $\mathrm{HNO}_{3}$ effervesces and stains gray. $\mathrm{HCl}$ fumes tarnish; halo $\mathrm{HgCl}_{2}$ does not wash off. KCN, some specimens tarnish gray to brown, others negative. $\mathrm{FeCl}_{3}$ instantly stains iridescent. $\mathrm{KOH}$ negative. $\mathrm{HgCl}_{2}$ stains brown to iridescent. Very sectile.

Altaite (PbTe).-Isometric; color galena-white; hardness $\mathrm{B}$. KOH neg. $\mathrm{HNO}_{3}$ effervesces and stains through iridescent to dark gray. $\mathrm{HgCl}_{2}$ neg. $\mathrm{HCl}$ quickly tarnishes iridescent. $\mathrm{KCN}$ negative. $\mathrm{FeCl}_{3}$ quickly tarnishes iridescent. $\mathrm{KOH}$ and $\mathrm{HgCl}_{2}$ negative. Cubic cleavage but less marked than galena; shows fewer triangular pits.

Galena $(\mathrm{PbS})$.--Isometric; color galena-white; hardness $\mathrm{B}$. $\mathrm{HNO}_{3}$, a small percentage of galena specimens effervesce and turn black. $\mathrm{HCl}$ tarnishes iridescent. $\mathrm{KCN}$ negative: $\mathrm{FeCl}_{3}$ stains iridescent; with galena areas in contact with bornite, reaction is negative. $\mathrm{KOH}$ and $\mathrm{HgCl}_{2}$ negative. Perfect cubic cleavage; many specimens show abundant triangular pits. Galena specimens that effervesce with $\mathrm{HNO}_{3}$ give off noticeable amounts of $\mathrm{H}_{2} \mathrm{~S}$.

\section{ANISOTROPIC}

Bismuth (Bi).-Hexagonal; color pinkish gray; hardness $\mathrm{A}-$. KOH neg. Strong anisotropism. $\mathrm{HNO}_{3}$ effervesces and darkens. $\mathrm{HCl}$ $\mathrm{HgCl}_{2} \quad$ stains differentially black; sometimes brings out lamellar structure. $\mathrm{KCN}$ negative. $\mathrm{FeCl}_{3}$ instantly stains iridescent. $\mathrm{KOH}$ negative. $\mathrm{HgCl}_{2}$ stains brown in spots; doubtful.

Telradymite $\left(\mathrm{Bi}_{2}(\mathrm{Te}, \mathrm{S})_{3}\right)$.- - Hexagonal; color silvery white; hardness $\mathrm{B}$. $\mathrm{HNO}_{8}$ effervesces and stains brown to black; action sometimes slow in starting. $\mathrm{HCl}$ stains light brown to iridescent; in some areas action is very slow. $\mathrm{KCN}$ negative. $\mathrm{FeCl}_{3}$ stains iridescent. $\mathrm{KOH}$ negative. $\mathrm{HgCl}_{2}$, some varieties tarnish iridescent; others negative. Some sulphur usually replaces tellurium in the molecule. A sulphur-free variety is known as telluro-bismutite. Perfect basal cleavage.

KOH neg. Malildite ( $\left.\left(\mathrm{Ag}_{2}, \mathrm{~Pb}\right) \mathrm{S} . \mathrm{Bi}_{2} \mathrm{~S}_{3}\right)$.-Color galena-white; hardness. C. $\mathrm{HgCl}_{2}$ neg. $\mathrm{KCN}$ negative. $\mathrm{FeCl}_{3}$ stains light brown. $\mathrm{KOH}$ and $\mathrm{HgCl}_{2}$ negative. Specimen examined is from O'Brien mine, Cobalt,

0ntario. Is intergrown with another mineral containing $\mathrm{Pb}, \mathrm{Bi}$, and $\mathrm{S}$ without 
any silver. Microchemical tests on the matildite indicate the presence of a sub. stantial amount of lead.

Tellurium (Te).-Hexagonal; color silvery white; hardness B. Strong anisa. tropism. $\mathrm{HNO}_{3}$ stains black with effervescence. $\mathrm{HCl}$ fumes tarnish. $\mathrm{KCl}$ negative. $\mathrm{FeCl}_{3}$ slowly stains light brown. $\mathrm{KOH}$ and $\mathrm{HgCl}_{2}$ negative.

Cosalite $\left(2 \mathrm{PbS} . \mathrm{Bi}_{2} \mathrm{~S}_{3}\right)$.-Monoclinic; color galena-white; hardness B. Strong anisotropism. $\mathrm{HNO}_{3}$ stains black with slow (doubtful) effervescence; fumb tarnish. $\mathrm{HCl}$ slowly stains differentially gray to brown. $\mathrm{KCN}$ negative. $\mathrm{FeC}_{4}$ stains light brown. $\mathrm{KOH}$ and $\mathrm{HgCl}_{2}$ negative. Prismatic. Specimen from Cobalt, Ontario; described and analyzed by T. L. Walker.

\section{$\mathrm{HNO}_{3}$ eff., $\mathrm{HCl}$, KCN neg., $\mathrm{FeCl}_{3}$ neg.}

\section{ISOTROPIC}

Alabandite (MnS).--Isometric; color gray, similar to sphal. KOH neg. erite; hardness C. $\mathrm{HNO}_{3}$ effervesces and gives off $\mathrm{H}_{2} \mathrm{~S}$; tar. $\mathbf{H g C l}_{2}$ neg. nishes brown; sometimes brings out grain structure. $\mathrm{HO}$ effervesces and gives off $\mathrm{H}_{2} \mathrm{~S}$; tarnishes black. $\mathrm{KCN}, \mathrm{FeCl}_{4}$ $\mathrm{KOH}$, and $\mathrm{HgCl}_{2}$ negative. Powder and internal reflection greenish yellow.

\section{ANISOTROPIC}

КОН

Jamesonite (4PbS.FeS. $\left.3 \mathrm{Sb}_{2} \mathrm{~S}_{3}\right)$. - Monoclinic; color galens. $\mathrm{HgCl}_{2}$ neg. iridescent with slow effervescence. $\mathrm{HCl}$ fumes tarnish. KCV and $\mathrm{FeCl}_{3}$ negative. KOH tarnishes iridescent. $\mathrm{HgCl}_{2}$ neg. tive. Prismatic. Perfect basal cleavage.

Plagionite (5 $\left.\mathrm{PbS} .4 \mathrm{Sb}_{2} \mathrm{~S}_{3}\right)$.-Monoclinic; color white; hardness C. HNO, effervesces and stains black. $\mathrm{HCl}$ fumes tarnish on some areas; other aress negative. $\mathrm{KCN}$ and $\mathrm{FeCl}_{3}$ negative. $\mathrm{KOH}$ stains gray to brown. $\mathrm{HgCl}_{8}$ nerative. Prismatic. Very rare. Distinguished from zinkenite only by crystal form.

Semseyite (9PbS.4 $\mathrm{Sb}_{2} \mathrm{~S}_{3}$ ).-Monoclinic; color gray; hardness C. Strong anisotropism. $\mathrm{HNO}_{3}$ effervesces and stains black. $\mathrm{HCl}$ fumes tarnish a little; stains slightly in places. $\mathrm{KCN}$ and $\mathrm{FeCl}_{3}$ negative. $\mathrm{KOH}$ slowly stains iridescent. $\mathrm{HgCl}_{2}$ negative. Prismatic.

Zinkenite $\left(\mathrm{PbS} . \mathrm{Sb}_{2} \mathrm{~S}_{3}\right)$.-Orthorhombic; color gray; hardness $\mathrm{C}$. $\mathrm{HNO}_{3}$ effervesces and darkens. $\mathrm{HCl}$ fumes tarnish slightly. $\mathrm{KCN}$ and $\mathrm{FeCl}_{3}$ negative $\mathrm{KOH}$ stains iridescent. $\mathrm{HgCl}_{2}$ negative. Prismatic. Has no distinct clearsge. Specimen from Wolfsberg, Harz, the type locality.

Troilite (FeS).-Hexagonal; color somewhat similar to pyrrhotite but mone yellowish; hardness D. Strong anisotropism. $\mathrm{HNO}_{3}$ effervesces violently and gives off $\mathrm{H}_{2} \mathrm{~S}$. $\mathrm{HCl}$ effervesces and gives off $\mathrm{H}_{2} \mathrm{~S}$. $\mathrm{KCN}$ and $\mathrm{FeCl}_{3}$ negative $\mathrm{KOH}$ stains differentially iridescent; slow. $\mathrm{HgCl}_{2}$ negative. Very rare but sometimes found in meteorites.

Bismuthinite $\left(\mathrm{Bi}_{2} \mathrm{~S}_{3}\right)$.-Orthorhombic; color galena-white; KOH neg. hardness $\mathrm{B}+$. Strong anisotropism. $\mathrm{HNO}_{3}$ slowly effervesces $\mathrm{HgCl}_{2}$ and stains black. HCl fumes tarnish. $\mathrm{KCN}, \mathrm{FeCl}$, and negative. Prismatic. $\mathrm{KOH}$ negative. $\mathrm{HgCl}_{2}$ stains light brown; some specimens

Bismutoplagionite ( $5 \mathrm{PbS}_{4} 4 \mathrm{Bi}_{2} \mathrm{~S}_{3}$ ).-Orthorhombic; color gray; KOH neg. hardness $\mathrm{B}+\mathrm{H} \mathrm{HNO}_{3}$ quickly stains iridescent; efferveses $\mathbf{H g C l}_{2}$ neg. slightly. $\mathrm{HCl}$ fumes tarnish; some areas stain iridescent; other negative. $\mathrm{KCN}, \mathrm{FeCl}_{3}, \mathrm{KOH}$, and $\mathrm{HgCl}_{2}$ negative. Prismatia Good cleavage in two directions. Reported only from Wickes, Mont. Analyzed by E. V. Shannon (U. S. Nat. Mus. Proc., vol. 58, p. 589, 1920). 
Boulangerite (5 $\left.\mathrm{PbS} .2 \mathrm{Sb}_{2} \mathrm{~S}_{3}\right)$.- Orthorhombic; color galena-white; hardness B+. Strong anisotropism. $\mathrm{HNO}_{3}$ effervesces; surface stains black; action sometimes slow in starting. $\mathrm{HCl}$ fumes tarnish slightly; doubtful. $\mathrm{KCN}$, $\mathrm{FeCl}_{3}, \mathrm{KOH}$, and $\mathrm{HgCl}_{2}$ negative. Prismatic. Distinguished from jamesonite by $\mathrm{KOH}$ test.

Meneghinite (4 $\left.\mathrm{PbS} \cdot \mathrm{Sb}_{2} \mathrm{~S}_{3}\right)$.-Orthorhombic; color white; hardness $\mathrm{C}-$. Strong anisotropism. $\mathrm{HNO}_{3}$ effervesces; surface stains black; usually reaction is slow in starting, but once started the etching rapidly advances as a wave from one side of the drop to the other. $\mathrm{HCl}$ fumes tarnish; some areas negative. $\mathrm{KCN}, \mathrm{FeCl}_{3}, \mathrm{KOH}$, and $\mathrm{HgCl}_{2}$ negative. Prismatic. Specimen examined is from Bottino, Tuscany, Italy, the type locality. Mineral is intergrown with galena, hence formula above given is open to question.

\section{$\mathrm{HNO}_{3}$ eff., $\mathrm{HCl}$ neg., $\mathrm{KCN}, \mathrm{FeCl}_{3}$}

\section{ISOTROPIC}

Copper $(\mathrm{Cu})$.-Isometric; color coppery red; hardness B.

KOH $\mathrm{HNO}_{3}$ effervesces without tarnishing. $\mathrm{HCl}$ negative; sometimes

$\mathrm{HgCl}_{2}$ tarnishes faintly. $\mathrm{KCN}$ slowly browns. $\mathrm{FeCl}_{3}$ quickly darkens. $\mathrm{KOH}$ slowly tarnishes brown to iridescent. $\mathrm{HgCl}_{2}$ quickly blackens.

Chalcocite $\left(\mathrm{Cu}_{2} \mathrm{~S}\right)$.- - Isometric and orthorhombic; color bluish коH neg. gray; hardness B. Hypogene chalcocite with more than 8 per $\mathrm{HgCl}_{2}$ cent of dissolved covellite is isotropic. Very fine grained supergene chalcocite is also apparently isotropic. Most chalcocite, both hypogene and supergene, is weakly anisotropic. $\mathrm{HNO}_{3}$ effervesces vigorously; mineral stains blue; with some isotropic varieties brings out octahedral etch cleavage; very fine grained supergene chalcocite, however, will not show etch cleavage. $\mathrm{HCl}$ negative; some varieties tarnish faintly. KCN quickly stains black; first brings out etch cleavage, but if drop is allowed to remain, etch cleavage is destroyed and a rough, corroded surface remains. $\mathrm{FeCl}_{3}$ stains blue; with some isotropic specimens brings out octahedral etch cleavage. $\mathrm{KOH}$ negative. $\mathrm{HgCl}_{2}$ tarnishes slightly.

Silver (Ag).-Isometric; color silvery white; hardness B. $\mathrm{HNO}_{3}$ effervesces slightly for a moment; surface stains gray. $\mathrm{HCl}$ fumes tarnish slightly; almost negative. $\mathrm{KCN}$ mineral tarnishes light gray to brown. $\mathrm{FeCl}_{3}$ instantly tarnishes iridescent. $\mathrm{KOH}$ negative. $\mathrm{HgCl}_{2}$ stains brown to iridescent. Very sectile.

Bornite $\left(\mathrm{Cu}_{5} \mathrm{FeS}_{4}\right)$.-Isometric; color pinkish brown; tarnished KOH neg. bornite is purple; hardness B. Usually isotropic, but some $\mathrm{HgCl}_{2}$ neg. specimens show weak anomalous anisotropism. $\mathrm{HNO}_{3}$ effervesces; surface stains yellowish brown; sometimes develops etch cleavage, which tends to form a bricklike pattern. $\mathrm{HCl}$ negative. $\mathrm{KCN}$ stains brown. $\mathrm{FeCl}_{3}$ stains orange. $\mathrm{KOH}$ and $\mathrm{HgCl}_{2}$ negative. Usually associated with chalcopyrite and other copper minerals.

\section{ANISOTROPIC}

Chalcocite $\left(\mathrm{Cu}_{2} \mathrm{~S}\right)$.- Isometric and orthorhombic; color bluish KOH neg. gray; hardness B. Most chalcocite is weakly anisotropic. $\mathrm{HgCl}_{2} \quad \mathrm{HNO}_{3}$ effervesces vigorously; mineral stains blue; with most varieties brings out parallel etch cleavage; very fine grained supergene chalcocite, however, will not show etch cleavage; with fine grained sooty chalcocite brings out grain boundaries only. $\mathrm{HCl}$ negative; some varieties tarnish faintly. KCN quickly stains black; first brings out etch cleavage, but if 
drop is allowed to remain, etch cleavage is destroyed, and a rough, corroded surface remains. $\mathrm{FeCl}_{3}$ stains blue; most specimens exhibit parallel etch cleavage $\mathrm{KOH}$ negative. $\mathrm{HgCl}_{2}$ tarnishes slightly.

Bornite $\left(\mathrm{Cu}_{5} \mathrm{FeS}_{4}\right)$. - Isometric; color pinkish brown; hardne KOH neg. B. Most specimens are isotropic, but some exhibit weak anom. $\mathrm{HgCl}_{2}$ neg. alous anisotropism. $\mathrm{HNO}_{3}$ effervesces; surface stains yellowish brown; sometimes develops etch cleavage, which tends to forms bricklike pattern. $\mathrm{KCN}$ stains brown. $\mathrm{FeCl}_{3}$ stains orange. $\mathrm{KOH}$ and $\mathrm{HgCl}_{4}$ negative. Usually associated with chalcopyrite and other copper minerals.

$\mathrm{HNO}_{3}$ eff., $\mathrm{HCl}$ neg., $\mathrm{KCN}, \mathrm{FeCl}_{3}$ neg.

\section{ISOTROPIC}

Electrum (alloy of $\mathrm{Au}$ and $\mathrm{Ag}$ ).--Isometric; color light yellow; KOH neg. hardness B. $\mathrm{HNO}_{3}$ fumes tarnish; effervesces slightly. $\mathrm{HC}$ $\mathrm{HgCl}_{2}$ negative. $\mathrm{KCN}$ quickly stains dark. $\mathrm{FeCl}_{3}$ and $\mathrm{KOH}$ negative. $\mathrm{HgCl}_{2}$ stains iridescent to black. Very sectile.

\section{ANISOTROPIC}

Klaprotholite $\left(3 \mathrm{Cu}_{2} \mathrm{~S}_{2} 2 \mathrm{Bi}_{2} \mathrm{~S}_{3}\right)$. - Probably isometric; color gras KOH neg. like tetrahedrite; in oblique light it has a pinkish brown tinge; $\mathbf{H g C l}_{2}$ neg. hardness C. Weak anisotropism, possibly anomalous. HNO, slowly stains brown; fumes tarnish; seems to be a slow efferver. cence. $\mathrm{HCl}$ negative. $\mathrm{KCN}$ stains differentially iridescent. $\mathrm{FeCl}_{3}, \mathrm{KOH}$, and $\mathrm{HgCl}_{2}$ negative. Specimen examined came from Butte, Mont. It was deter. mined by F. B. Laney as klaprotholite. It is intergrown with covellite in mutusl boundary and subgraphic structures.

\section{$\mathrm{HNO}_{3}$ eff., $\mathrm{HCl}$ neg., $\mathrm{KCN}$ neg., $\mathrm{FeCl}_{3}$}

\section{ANISOTROPIC}

Arsenic (native) (As).-Hexagonal; color gray; hardness C. KOH neg. $\mathrm{HNO}_{3}$ slowly effervesces; surface stains iridescent. $\mathrm{HCl}$ and $\mathrm{HgCl}_{2} \quad \mathrm{KCN}$ negative. $\mathrm{FeCl}_{3}$ quickly stains iridescent to black. $\mathrm{KOH}$ negative. $\mathrm{HgCl}_{2}$ slowly stains light brown; some areas almost negative. Good basal cleavage. Gives powder when scratched.

Tellurium (Te).-Color silvery white; hardness $\mathrm{B}+\mathrm{H} \mathrm{HNO}_{3}$ tarnishes bladk with effervescence. $\mathrm{HCl}$, some areas, fumes tarnish; other areas negative KCN negative. $\mathrm{FeCl}_{3}$ stains faintly yellow. $\mathrm{KOH}$ negative. $\mathrm{HgCl}_{2}$, some areas stain light brown, others negative.

Tetradymite $\left(\mathrm{Bi}_{2}(\mathrm{Te}, \mathrm{S})_{3}\right)$.- - Hexagonal; color galena-white; hardness $\mathrm{B}+$. $\mathrm{HNO}_{3}$ effervesces; surface stains brown to black; action sometimes slow in starting. $\mathrm{HCl}$ slowly stains light brown to iridescent; some areas negative. $\mathrm{KCN}$ negative. $\mathrm{FeCl}_{3}$ stains iridescent. $\mathrm{KOH}$ negative. $\mathrm{HgCl}_{2}$, some spectmens stain iridescent; others negative. Perfect basal cleavage.

Aikinite $\left(\mathrm{Cu}_{2} \mathrm{~S} .2 \mathrm{PbS} . \mathrm{Bi}_{2} \mathrm{~S}_{3}\right)$. - Orthorhombic; color whit';

KOH neg. hardness C. Strong anisotropism. $\mathrm{HNO}_{3}$ effervesces; surface $\mathbf{H g C l}_{2}$ neg. tarnishes iridescent to black. $\mathrm{HCl}$ and $\mathrm{KCN}$ negative. $\mathrm{FeO}_{4}$ stains light brown and brings out grain structure; action very slow and effect slight. $\mathrm{KOH}$ and $\mathrm{HgCl}_{2}$ negative.

Calaverite (( $\left.\mathrm{Au}, \mathrm{Ag}) \mathrm{Te}_{2}\right)$.-Monoclinic; color light yellow; hardness C. Strong anisotropism. $\mathrm{HNO}_{3}$ effervesces; surface stains black. $\mathrm{HCl}$ and $\mathrm{KCN}$ negative. 
$\mathrm{FeCl}_{3}$, most specimens negative; some stain slowly light brown. $\mathrm{KOH}$ and $\mathrm{HgCl}_{2}$ negative. Prismatic.

Galenobismutite ( $\mathrm{PbS} \cdot \mathrm{Bi}_{2} \mathrm{~S}_{3}$ ).-Color galena-white; hardness $\mathrm{B}$. $\mathrm{HNO}_{3}$ effervesces and leaves yellow deposit. $\mathrm{HCl}$ and $\mathrm{KCN}$ negative. $\mathrm{FeCl}_{3}$ leaves slight yellow stain; almost negative. $\mathrm{KOH}$ and $\mathrm{HgCl}_{2}$ negative. Specimen is from Fahlun, Sweden. Gives microchemical tests for $\mathrm{Pb}, \mathrm{Bi}, \mathrm{S}$, and Se. Intergrown with galena.

Melonite $\left(\mathrm{Ni}_{2} \mathrm{Te}_{3}\right)$.-Color pale yellow; hardness $\mathrm{B}$. $\mathrm{HNO}_{3}$ effervesces; surface stains black. $\mathrm{HCl}$ and $\mathrm{KCN}$ negative. $\mathrm{FeCl}_{3}$ stains dark brown. $\mathrm{KOH}$ and $\mathrm{HgCl}$ negative. Very rare. A polished surface when tilted so as to get the reflection of daylight and viewed with the unaided eye has a distinctly pink tinge, about that of bismuth.

Sylvanite (( $\left.\mathrm{Au}, \mathrm{Ag}) \mathrm{Te}_{2}\right)$.-Monoclinic; color creamy gray; hardness $\mathrm{C}$. $\mathrm{HNO}_{3}$ slowly effervesces; surface stains iridescent; effervescence doubtful. $\mathrm{HCl}$ and $\mathrm{KCN}$ negative. $\mathrm{FeCl}_{3}$ sometimes leaves light-yellow stain; most areas negative. $\mathrm{KOH}$ and $\mathrm{HgCl}_{2}$ negative. Lighter in color than calaverite.

\section{$\mathrm{HNO}_{3}$ eff., $\mathrm{HCl}$ neg., $\mathrm{KCN}$ neg., $\mathrm{FeCl}_{3}$ neg.}

\section{ISOTROPIC}

Polydymite $\left(\mathrm{Ni}_{3} \mathrm{~S}_{4}\right)$. - Isometric; color brass-yellow; hardness $\mathrm{F}$. KOH neg. $\mathrm{HNO}_{3}$, slow effervescence; fumes tarnish; mineral slowly stains $\mathrm{HgCl}_{2}$ neg. iridescent. $\mathrm{HCl}$, drop turns yellow; mineral unaffected. $\mathrm{KCN}$, $\mathrm{FeCl}_{3}, \mathrm{KOH}$, and $\mathrm{HgCl}_{2}$ negative. Color about same as that of pentlandite. The only genuine polydymite seen by the writer comes from Grunau, Saxony; the other specimens of so-called polydymite from Sudbury and elsewhere have proved to be violarite.

Sphalerite (ZnS).-Isometric; color gray; hardness C-. $\mathrm{HNO}_{3}$ fumes tarnish; more rarely slow effervescence can be observed. $\mathrm{HCl}$, drop sometimes turns yellow; mineral unaffected. $\mathrm{KCN}, \mathrm{FeCl}_{3}, \mathrm{KOH}$, and $\mathrm{HgCl}_{2}$ negative. Some specimens show resin-colored internal reflection. Powder white to brown, depending on proportion of iron. Some specimens exhibit triangular pits from dodecahedral cleavage.

Violarite $\left((\mathrm{Ni}, \mathrm{Fe})_{3} \mathrm{~S}_{4}\right)$.- Isometric; colòr violet; hardness C. $\mathrm{HNO}_{3}$ slowly effervesces; surface stains slowly brown. $\mathrm{HCl}, \mathrm{KCN}, \mathrm{FeCl}_{3}, \mathrm{KOH}$, and $\mathrm{HgCl}_{2}$ negative. Some specimens exhibit triangular pits and cubic cleavage. Mineral is unstable and usually crumbly. Associated with and usually a supergene replacement product of pentlandite or pyrrhotite.44

\section{ANISOTROPIC}

KOH

Jamesonite (4PbS.FeS.3Sb $\mathrm{S}_{3}$ ).-Monoclinic; color galena-

$\mathrm{HgCl}_{2}$ white; hardness B. Strong anisotropism. $\mathrm{HNO}_{3}$ tarnishes iridescent with slow effervescence. $\mathrm{HCl}$ fumes usually tarnish; sometimes negative. $\mathrm{KCN}$ and $\mathrm{FeCl}_{3}$ negative. $\mathrm{KOH}$ stains differentially iridescent. $\mathrm{HgCl}_{2}$ negative. Prismatic.

Plagionite - $\left(5 \mathrm{PbS} .4 \mathrm{Sb}_{2} \mathrm{~S}_{3}\right)$.-Monoclinic; color gray; hardness C. $\mathrm{HNO}_{3}$ effervesces; surface stains iridescent. $\mathrm{HCl}$ negative; on some areas fumes tarnish slightly. $\mathrm{KCN}$ and $\mathrm{FeCl}_{3}$ negative. $\mathrm{KOH}$ stains gray to brown. $\mathrm{HgCl}_{2}$ negative. Distinguished from zinkenite only by its crystal form. Prismatic. Rare.

\footnotetext{
H See Short, M. N., and Shannon, E. V., Violarite and other rare nickel sulphides: Am. Mineralogist, vol. 15. pp. 2-7, 1930.
} 
Rathite (3PbS.2 $\left.\mathrm{As}_{2} \mathrm{~S}_{3}\right)$.-Orthorhombic; color galena-white; hardness C. Weak anisotropism. $\mathrm{HNO}_{3}$ effervesces slowly and stains black; effervescence not always observed. $\mathrm{HCl}, \mathrm{KCN}$, and $\mathrm{FeCl}_{3}$ negative. $\mathrm{KOH}$ stains brown. $\mathrm{HgCl}_{2}$ negative. Very rare; reported only from Binnenthal, Switzerland.

Aikinite (CuS.2PbS. $\left.\mathrm{Bi}_{2} \mathrm{~S}_{3}\right)$.-Orthorhombic; color galens. KОH neg. white; hardness C. Strong anisotropism. $\mathrm{HNO}_{3}$ slowly effer. $\mathrm{HgCl}_{2}$ neg. vesces; surface stains black. $\mathrm{HCl}, \mathrm{KCN}, \mathrm{FeCl}_{3}, \mathrm{KOH}$, and $\mathrm{HgCl}_{2}$ negative.

Bismuthinite $\left(\mathrm{Bi}_{2} \mathrm{~S}_{3}\right)$.- Orthorhombic; color galena-white; hardness B. Strong anisotropism. $\mathrm{HNO}_{3}$ effervesces; surface stains iridescent. $\mathrm{HCl}, \mathrm{KCN}, \mathrm{FeCl}$, $\mathrm{KOH}$, and $\mathrm{HgCl}_{2}$ negative. Prismatic.

Boulangerite ( $\left.5 \mathrm{PbS} .2 \mathrm{Bi}_{2} \mathrm{~S}_{3}\right)$.- Orthorhombic; color galena-white; hardness $\mathrm{B}$. Strong anisotropism. $\mathrm{HNO}_{3}$ effervesces; surface stains black; action often slow in starting. $\mathrm{HCl}, \mathrm{KCN}, \mathrm{FeCl}_{3}, \mathrm{KOH}$, and $\mathrm{HgCl}_{2}$ negative. Prismatic. Dis. tinguished from jamesonite by $\mathrm{KOH}$ test.

Calaverite (( $\left.\mathrm{Au}, \mathrm{Ag}) \mathrm{Te}_{2}\right)$.--Monoclinic; color light yellow; hardness C. Strong anisotropism. $\mathrm{HNO}_{3}$ effervesces; surface stains black. $\mathrm{HCl}$ and $\mathrm{KCN}$ negar. tive. $\mathrm{FeCl}_{3}$, most specimens negative; some stain slowly light brown. $\mathrm{KOH}$ and $\mathrm{HgCl}_{2}$ negative. Prismatic.

Emplectite $\left(\mathrm{Cu}_{2} \mathrm{~S} . \mathrm{Bi}_{2} \mathrm{~S}_{3}\right)$.-Orthorhombic; color creamy; hardness C. Strong anisotropism. $\mathrm{HNO}_{3}$ slowly effervesces; surface stains light brown. $\mathrm{HCl}, \mathrm{KCN}$, $\mathrm{FeCl}_{3}, \mathrm{KOH}$, and $\mathrm{HgCl}_{2}$ negative. Rare.

Benjaminite ((Cu, $\left.\mathrm{Ag})_{2} \mathrm{~S} .2 \mathrm{PbS} .2 \mathrm{Bi}_{2} \mathrm{~S}_{3}\right)$. - Orthorhombic; color white; hardness B. Shows twinning. $\mathrm{HNO}_{3}$ quickly turns KOH neg. black with effervescence. $\mathrm{HCl}$ negative; fumes tarnish slightly. $\mathbf{H g C l}_{2}$ neg. $\mathrm{KCN}, \mathrm{FeCl}_{3}, \mathrm{KOH}$, and $\mathrm{HgCl}_{2}$ negative. Good cleavage in one direction. Reported only from Outlaw mine, Manhattan, Nev. Analyzed by E. V. Shannon (U. S. Nat. Mus. Proc., vol. 65, art. 24, p.1, 1924).

Geocronite $\left(5 \mathrm{PbS} . \mathrm{Sb}_{2} \mathrm{~S}_{3}\right)$.-Orthorhombic; color galena-white; hardness $\mathrm{B}$. $\mathrm{HNO}_{3}$ stains black and effervesces slowly; effervescence not always apparent. $\mathrm{HCl}, \mathrm{KCN}, \mathrm{FeCl}_{3}, \mathrm{KOH}$, and $\mathrm{HgCl}_{2}$ negative. Prismatic. Rare.

Guanajuatite $\mathrm{Bi}_{2}(\mathrm{Se}, \mathrm{S})_{3}$.- Orthorhombic; color gray; hardness $\mathrm{B}-$. Strong anisotropism. $\mathrm{HNO}_{3}$ slowly effervesces and stains iridescent; fumes tarnish; effervescence not always observed. $\mathrm{HCl}, \mathrm{KCN}, \mathrm{FeCl}_{3}, \mathrm{KOH}$, and $\mathrm{HgCl}_{2}$ negative. Prismatic. Rare.

Krennerite $\left((\mathrm{Ag}, \mathrm{Au}) \mathrm{Te}_{2}\right)$.-Orthorhombic; color creamy white; hardness C. Strong anisotropism. $\mathrm{HNO}_{3}$ slowly effervesces; surface stains iridescent. $\mathrm{HCl}$, $\mathrm{KCN}, \mathrm{FeCl}_{3}, \mathrm{KOH}$, and $\mathrm{HgCl}_{2}$ negative. Prismatic. Lighter in color than calaverite.

Meneghinite (4PbS. $\mathrm{Sb}_{2} \mathrm{~S}_{3}$ ).-Orthorhombic; color galena-white; hardness $\mathrm{C}$. Strong anisotropism. $\mathrm{HNO}_{3}$ effervesces; surface stains black; usually reaction is slow in starting, but once started it rapidly advances from one side of the drop to the other. $\mathrm{HCl}$, fumes tarnish; some areas negative. $\mathrm{KCN}, \mathrm{FeCh}$ $\mathrm{KOH}$, and $\mathrm{HgCl}_{2}$ negative. Prismatic.

Rezbanyite ( $\left.2 \mathrm{PbS} 3 \mathrm{Bi}_{2} \mathrm{~S}_{3}\right)$. - Crystal system unknown; color galena-white; hardness C. Strong anisotropism. $\mathrm{HNO}_{3}$ effervesces strongly; surface turns black. $\mathrm{HCl}, \mathrm{KCN}, \mathrm{FeCl}_{3}, \mathrm{KOH}$, and $\mathrm{HgCl}_{2}$ negative. Specimen examined is from Rezbanya, Hungary, the type locality. Distinguished from other lead. bismuth compounds by the lack of prismatic habit. Rare.

Sylvanite (( $\left.\mathrm{Au}, \mathrm{Ag}) \mathrm{Te}_{2}\right)$.-Monoclinic; color creamy gray; hardness C. $\mathrm{HNO}_{3}$ tarnishes iridescent with slow (doubtful) effervescence. $\mathrm{HCl}, \mathrm{KCN}, \mathrm{FeCl}_{3}, \mathrm{KOH}$, and $\mathrm{HgCl}_{2}$ negative. Distinguished from krennerite only by crystal form. 


\title{
$\mathrm{HNO}_{3}, \mathrm{HCl}, \mathrm{KCN}, \mathrm{FeCl}_{3}$
}

\author{
ISOTROPIC
}

Antimony (Sb).-Hexagonal; color silvery white; hardness B. кон neg. Apparently isotropic. $\mathrm{HNO}_{3}$ quickly stains black. $\mathrm{HCl}$ fumes $\mathrm{HgCl}_{2}$ tarnish slightly and surface stains after long etching; practically negative. $\mathrm{KCN}$ stains light brown. $\mathrm{FeCl}_{3}$ stains black. $\mathrm{KOH}$ negative. $\mathrm{HgCl}_{2}$ quickly stains iridescent.

Argentite $\left(\mathrm{Ag}_{2} \mathrm{~S}\right)$.- - Isometric; color gray; hardness $\mathrm{A}$. Most specimens are isotropic, but some show weak anomalous anistropism. $\mathrm{HNO}_{3}$ slowly etches differentially. $\mathrm{HCl}$ fumes tarnish; aureole will not wash off; this is the most distinctive reaction for argentite. $\mathrm{KCN}$ quickly blackens. $\mathrm{FeCl}_{3}$ quickly stains black. $\mathrm{KOH}$ negative. $\mathrm{HgCl}_{2}$ quickly iridescent. Sectile.

Dyscrasite $\left(\mathrm{Ag}_{3} \mathrm{Sb}\right)$. - Isometric; color silvery white; hardness $\mathrm{C}-\mathrm{HNO}_{3}$ quickly stains black; with some specimens brings out grating structure. $\mathrm{HCl}$, some areas seem to stain a little; others doubtful or negative. KCN stains differentially and brings out structure. $\mathrm{FeCl}_{3}$ quickly stains iridescent. $\mathrm{KOH}$ negative. $\mathrm{HgCl}_{2}$ stains brown and brings out structure. Almost sectile but yields powder when scratched.

Hessite ( $\mathrm{Ag}_{2} \mathrm{Te}$ ).-Isometric; color gray; hardness A. Some specimens are isotropic; others show anomalous anisotropism. Some specimens show both isotropic and anisotropic areas on the same surface. $\mathrm{HNO}_{3}$ stains iridescent to black. HCl slowly stains black; negative on small areas. KCN slowly stains black; weak. $\mathrm{FeCl}_{3}$ quickly stains iridescent. $\mathrm{KOH}$ negative. $\mathrm{HgCl}_{2}$ tarnishes light brown.

\section{ANISOTROPIC}

Stephanite (5 $\left.\mathrm{Ag}_{2} \mathrm{~S} . \mathrm{Sb}_{2} \mathrm{~S}_{3}\right)$.-Orthorhombic; color gray; hard-

КОН ness $\mathrm{B}$. $\mathrm{HNO}_{3}$ practically negative; after long etching fumes

$\mathrm{HgCl}_{2}$ tarnish faintly and mineral seems to stain very light brown; reaction doubtful. $\mathrm{HCl}$ fumes tarnish; halo will not wash off. $\mathrm{KCN}$ stains dark gray and brings out scratches. $\mathrm{FeCl}_{3}$ tarnishes. $\mathrm{KOH}$ quickly stains black. $\mathrm{HgCl}_{2}$ quickly stains iridescent. Metallic powder.

Argentite $\left(\mathrm{Ag}_{2} \mathrm{~S}\right)$.- - Isometric; color gray; hardness A. Some коH neg. specimens are isotropic; others show anomalous anisotropism. $\mathrm{HgCl}_{2} \mathrm{HNO}_{3}$ fumes tarnish. $\mathrm{HCl}$ fumes tarnish; halo does not wash off. KCN stains differentially black and brings out structure. $\mathrm{FeCl}_{3}$ quickly stains black. $\mathrm{KOH}$ negative. $\mathrm{HgCl}_{2}$ quickly stains black. Very sectile; does not yield powder.

Hessite $\left(\mathrm{Ag}_{2} \mathrm{Te}\right)$.- - Isometric; color gray; hardness $\mathrm{A}+$. Some specimens are isotropic; others show anomalous anisotropism. Some specimens show both isotropic and anisotropic areas on the same polished surface. $\mathrm{HNO}_{3}$ quickly stains black. $\mathrm{HCl}$ slowly stains black. KCN stains dark in spots; weak. $\mathrm{FeCl}_{3}$ instantly tarnishes iridescent. $\mathrm{KOH}$ negative. $\mathrm{HgCl}_{2}$ stains brown. Closely resembles argentite in appearance, but hessite will yield powder when scratched; argentite yields only metallic shavings.

Stromeyerite $\left((\mathrm{Cu}, \mathrm{Ag})_{2} \mathrm{~S}\right)$.-Orthorhombic; color gray; hardness B. Strong anisotropism. $\mathrm{HNO}_{3}$ stains through iridescent to black. $\mathrm{HCl}$ fumes tarnish; halo does not wash off; action sometimes almost negative. KCN quickly stains black. $\mathrm{FeCl}_{3}$ quickly stains iridescent. $\mathrm{KOH}$ negative. $\mathrm{HgCl}_{2}$ stains iridescent. Closely resembles argentite in color and etch tests; stromeyerite has stronger anisotropism and yields a powder when scratched.

Umangite $\left(\mathrm{Cu}_{3} \mathrm{Se}_{2}\right)$.-Color purple, similar to slightly tarnished bornite; hardness B. Strongly anisotropic; gives four extinctions; interference color is a fiery 
orange very similar to that of klockmannite. $\mathrm{HNO}_{3}$ fumes tarnish; surface tum blue. $\mathrm{HCl}$, surface turns blue. KCN, surface tarnishes differentially blue to dark gray, bringing out grain structure. $\mathrm{FeCl}_{3}$, surface turns bluish. $\mathrm{KOH}$ negative. $\mathrm{HgCl}_{2}$, surface turns bluish. Associated with clausthalite and klock. mannite.

$\mathrm{HNO}_{3}, \mathrm{HCl}, \mathrm{KCN}, \mathrm{FeCl}_{3}$ neg.

ANISOTROPIC

Кон

$\mathrm{HgCl}_{2}$

Stephanite (5 $\left.\mathrm{Ag}_{2} \mathrm{~S}_{2} \mathrm{Sb}_{2} \mathrm{~S}_{3}\right)$. -Orthorhombic; color gray; hard. ness B. $\mathrm{HNO}_{3}$ practically negative; after long etching fumes tarnish faintly and mineral stains very light brown; reaction doubtful. $\mathrm{HCl}$ fumes tarnish; halo will not wash off. $\mathrm{KCV}$ quickly stains black. $\mathrm{FeCl}$, some specimens tarnish; others negative. $\mathrm{KOH}$ some specimens stain black; others negative. $\mathrm{HgCl}_{2}$ stains brown to iridescent, Metallic powder.

KOH Very strong anisotropism. $\mathrm{HNO}_{3}$ stains differentially iridescent. $\mathbf{H g C l}_{2}$ neg. $\mathrm{HCl}$ fumes sometimes tarnish slightly; usually reaction is nege. tive. KCN brings out scratches and sometimes stains slightly. $\mathrm{FeCl}_{3}$ negative. $\mathrm{KOH}$ instantly tarnishes brown and gives yellow coating; this is the most characteristic etch reaction for stibnite. $\mathrm{HgCl}_{2}$ negative. Prismatic, Hardness and color vary somewhat with orientation, giving mottled appearance that suggests the presence of more than one mineral.

\section{$\mathrm{HNO}_{3}, \mathrm{HCl}, \mathrm{KCN}$ neg., $\mathrm{FeCl}_{3}$}

\section{ISOTROPIC}

Aguilarıte $\left(\mathrm{Ag}_{2}(\mathrm{Se}, \mathrm{S})\right)$.- Isometric; color gray; hardness $\mathrm{A}$ KOH neg. $\mathrm{HNO}_{3}$ slowly stains light brown. $\mathrm{HCl}$ usually negative; fumes $\mathrm{HgCl}_{2}$ sometimes tarnish. KCN negative. $\mathrm{FeCl}_{3}$ etches iridescent, $\mathrm{KOH}$ negative. $\mathrm{HgCl}_{2}$ stains iridescent. Sectile. Rare.

Petzite (( $\left.\mathrm{Au}, \mathrm{Ag})_{2} \mathrm{Te}\right)$.- Isometric; color gray; hardness A. $\mathrm{HNO}_{3}$ quickly stains iridescent. $\mathrm{HCl}$ stains iridescent; some specimens negative. $\mathrm{KCN}$ negative. $\mathrm{FeCl}_{3}$ quickly stains iridescent. $\mathrm{KOH}$ negative. $\mathrm{HgCl}_{2}$ slowly stains brown. Frequently associated with hessite and native gold.

Clausthalite (PbSe).--Isometric; color galena-white; hardness KOH neg. $\mathrm{A}-\mathrm{H} \mathrm{HNO}_{3}$ tarnishes and leaves a coating which is brick-red $\mathbf{H g C l}_{2}$ neg. in oblique light; this distinguishes the mineral from galens. $\mathrm{HCl}$ slowly tarnishes light brown. $\mathrm{KCN}$ negative. $\mathrm{FeCl}_{8}$ tar nishes iridescent. $\mathrm{KOH}$ and $\mathrm{HgCl}_{2}$ negative. Has cubic cleavage less perfect than galena. Usually shows individual triangular pits here and there.

Galena (PbS).--Isometric; color galena-white; hardness $\mathrm{B}$. $\mathrm{HNO}_{3}$ stains black. HCl tarnishes brown to iridescent. $\mathrm{KCN}$ negative. $\mathrm{FeCl}_{3}$ tarnishes iridescent; this reaction is negative when galena is in contact with bornite. $\mathrm{KOH}$ and $\mathrm{HgCl}_{2}$ negative. Perfect cubic cleavage. Most specimens show paralled triangular pits.

\section{ANISOTROPIC}

Cylindrite $\left(6 \mathrm{PbS} \mathrm{Sb}_{2} \mathrm{~S}_{3} .6 \mathrm{SnS}_{2}\right)$.-Tetragonal; color gray; hard.

КOH

$\mathbf{H g C l}_{2}$ neg. stains iridescent. $\mathrm{HCl}$ fumes tarnish; stains slightly after etching two minutes. $\mathrm{KCN}$ negative. $\mathrm{FeCl}_{3}$ stains slightly light brown; doubtful. KOH stains light gray; in places iridescent. $\mathrm{HgCl}_{2}$ negative. Basd sections are more or less cylindrical and are made up of concentric shells. 
Hessite $\left(\mathrm{Ag}_{2} \mathrm{Te}\right)$.-Isometric; color gray, resembles argentite; KOH neg. hardness A. Most specimens show anomalous anisotropism. $\mathrm{HgCl}_{2}$. $\mathrm{HNO}_{3}$ stains black. $\mathrm{HCl}$ negative; fumes seem to tarnish faintly on some areas. $\mathrm{KCN}$ negative. $\mathrm{FeCl}_{3}$ stains iridescent. $\mathrm{KOH}$ negative. $\mathrm{HgCl}_{2}$ stains brown to iridescent. Almost sectile but yields powder when scratched.

Alaskaite ( $\left.\mathrm{Ag}_{2} \mathrm{~S} . \mathrm{PbS} .2 \mathrm{Bi}_{2} \mathrm{~S}_{3}\right)$.-Orthorhombic; color gray; hardкОH neg. ness C. Strong anisotropism. $\mathrm{HNO}_{3}$ slowly stains dark. $\mathrm{HCl}$ $\mathrm{HgCl}_{2}$ neg. fumes tarnish a little; doubtful. KCN negative. $\mathrm{FeCl}_{3}$ stains iridescent. $\mathrm{KOH}$ and $\mathrm{HgCl}_{2}$ negative. Argentiferous galenobismutite. Prismatic. Rare. Two analyses quoted by Dana give Ag 3 and 8 per cent.

Cosalite $\left.2 \mathrm{PbS} \mathrm{Bi}_{2} \mathrm{~S}_{3}\right)$.-Monoclinic, color galena-white; hardness $\mathrm{B}$. Strong anisotropism. $\mathrm{HNO}_{3}$ stains black with slow (doubtful) effervescence. $\mathrm{HCl}$ slowly stains differentially gray to black. $\mathrm{KCN}$ negative. $\mathrm{FeCl}_{3}$ stains light brown. $\mathrm{KOH}$ and $\mathrm{HgCl}_{2}$ negative. Prismatic. Specimen from Boston Creek mine, Cobalt, Ontario, described and analyzed by T. L. Walker.

Schapbachite ( $\left.\mathrm{Ag}_{2} \mathrm{~S} . \mathrm{PbS} . \mathrm{Bi}_{2} \mathrm{~S}_{3}\right)$.-Orthorhombic; color gray; hardness $\mathrm{B}-$. $\mathrm{HNO}_{3}$ slowly stains brown. $\mathrm{HCl}$ stains differentially; some areas unaffected. KCN negative. $\mathrm{FeCl}_{3}$ stains iridescent. $\mathrm{KOH}$ and $\mathrm{HgCl}_{2}$ negative. From Schapbach, Baden, the only locality reported. Differs from alaskaite in its higher silver content (Ag 11. 6 per cent).

\section{$\mathrm{HNO}_{3}, \mathrm{HCl}$, KCN neg., $\mathrm{FeCl}_{3}$ neg.}

\section{ISOTROPIC}

Hauerite $\left(\mathrm{MnS}_{2}\right)$.-Isometric; color gray; hardness E. HNO KOH neg. fumes tarnish slowly; some areas practically negative. $\mathrm{HCl}$ $\mathrm{HgCl}_{2}$ neg. solution turns brown; mineral stains light brown; fumes tarnish; some areas do not stain. $\mathrm{KCN}, \mathrm{FeCl}_{3}, \mathrm{KOH}$, and $\mathrm{HgCl}_{2}$ negative. $\mathrm{H}_{2} \mathrm{O}_{2}$, slow effervescence; surface does not stain. Cubic cleavage; surface shows triangular pits. Red powder.

Sphalerite (ZnS).-Isometric; color gray; hardness C. $\mathrm{HNO}_{3}$ fumes tarnish; on areas smaller than the drop, reaction is usually negative. $\mathrm{HCl}$ fumes sometimes tarnish; usually negative. $\mathrm{KCN}, \mathrm{FeCl}_{3}, \mathrm{KOH}$, and $\mathrm{HgCl}_{2}$ negative. Some specimens show resin-colored internal reflection. Dodecahedral cleavage; some specimens show triangular pits. Powder white to brown; if proportion of iron is high, powder is metallic.

Voltzite (4ZnS.ZnO).-Isometric; color gray, like sphalerite; hardness D. $\mathrm{HNO}_{3}$ fumes tarnish strongly. $\mathrm{HCl}$ tarnishes and gives off $\mathrm{H}_{2} \mathrm{~S}$. $\mathrm{KCN}, \mathrm{FeCl}_{3}$, $\mathrm{KOH}$, and $\mathrm{HgCl}_{2}$ negative. Resin-colored powder and internal reflection. No cleavage.

\section{ANISOTROPIC}

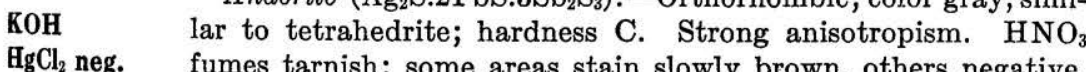

Andorite $\left(\mathrm{Ag}_{2} \mathrm{~S} .2 \mathrm{PbS} .3 \mathrm{Sb}_{2} \mathrm{~S}_{3}\right)$.-Orthorhombic; color gray, simifumes tarnish; some areas stain slowly brown, others negative. $\mathrm{HCl}$ fumes tarnish faintly. $\mathrm{KCN}$ and ${ }^{\top} \mathrm{FeCl}_{3}$ negative. $\mathrm{KOH}$ slowly stains brown. $\mathrm{HgCl}_{2}$ negative. Aqua regia effervesces vigorously and stains black. Almost invariably associated with stannite. Rare. Prismatic.

Cylindrite $\left(6 \mathrm{PbS} \mathrm{Sb}_{2} \mathrm{~S}_{3} \cdot 6 \mathrm{SnS}_{2}\right)$.-Tetragonal; color gray; hardness $\mathrm{C}-$. Strong anisotropism. $\mathrm{HNO}_{3}$ fumes tarnish; slowly stains iridescent. $\mathrm{HCl}$ fumes tarnish; stains slightly after etching two minutes. $\mathrm{KCN}$ negative. $\mathrm{FeCl}_{3}$ stains slightly brown; doubtful. $\mathrm{KOH}$ stains light gray, in places iridescent. $\mathrm{HgCl}$ negative. Basal sections are more or less cylindrical and are made up of concentric shells. 
Franckeite $\left(5 \mathrm{PbS} . \mathrm{Sb}_{2} \mathrm{~S}_{3} \cdot 2 \mathrm{SnS}_{2}\right)$.-Color gray; hardness $\mathrm{B} . \mathrm{HNO}_{3}$ stains differ. entially iridescent; fumes tarnish. $\mathrm{HCl}$ fumes tarnish slightly after long etch. ing; doubtful. $\mathrm{KCN}$ and $\mathrm{FeCl}_{3}$ negative. $\mathrm{KOH}$, some areas stain slightly; others negative. $\mathrm{HgCl}_{2}$ negative.

Sternbergite ( $\left.\mathrm{AgFe}_{2} \mathrm{~S}_{3-4}\right)$. - Orthorhombic; color light brown; hardness B. Verg strong anisotropism; shows without analyzing nicol. $\mathrm{HNO}_{3}$ fumes tarnish, $\mathrm{HCl}$ fumes tarnish slowly. $\mathrm{KCN}$ and $\mathrm{FeCl}_{3}$ negative. $\mathrm{KOH}$ stains differen. tially iridescent. $\mathrm{HgCl}_{2}$ negative; gives a deceptive "etching," owing to depasition of $\mathrm{HgCl}_{2}$ erystals. Habit prismatic. Usually associated with ruby silve minerals.

\section{KOH neg.} $\mathrm{HgCl}_{2}$ neg.

Bismutoplagionite (5PbS.4 $\left.4 \mathrm{Bi}_{2} \mathrm{~S}_{3}\right)$.-Orthorhombic; color gray; hardness $\mathrm{B}+\mathrm{HNO}_{3}$ quickly stains iridescent; effervesces slightly. $\mathrm{HCl}$ fumes tarnish; some areas stain iridescent; others negative. $\mathrm{KCN}, \mathrm{FeCl}_{3}, \mathrm{KOH}$, and $\mathrm{HgCl}_{2}$ negative. Prismatic. Good cleavage in two directions. Reported only from Wickes, Mont. Analyzed by E. V. Shannon (U. S. Nat. Mus. Proc., vol. 58, p. 600, 1920),

Cuprodescloizite $\left(\mathrm{Pb}_{2}(\mathrm{Zn}, \mathrm{Cu})_{2} \cdot \mathrm{V}_{2} \mathrm{O}_{9} \cdot \mathrm{H}_{2} \mathrm{O}\right)$. - Orthorhombic; color gray, similar to sphalerite; hardness $\mathrm{D}$. Weak anisotropism. $\mathrm{HNO}_{3}$ etches and deposits yellow powder. $\mathrm{HCl}$ etches and deposits yellow powder. $\mathrm{KCN}, \mathrm{FeCl}_{3}, \mathrm{KOH}_{1}$ and $\mathrm{HgCl}_{2}$ negative. Resinlike powder. Reddish-brown internal reflection.

Owyheeite (8PbS.2Ag $\left.2.5 \mathrm{Sb}_{2} \mathrm{~S}_{3}\right)$. - Orthorhombic; color gray; hardness $\mathrm{B}+$. Strong anisotropism. $\mathrm{HNO}_{3}$ stains differentially iridescent. $\mathrm{HCl}$ fumes tarnish. $\mathrm{KCN}, \mathrm{FeCl}_{3}, \mathrm{KOH}$, and $\mathrm{HgCl}_{2}$ negative. Prismatic structure. Shows triangular cleavage pits. Very rare. Specimen is from Poorman mine, Silver City districh Idaho; analyzed by E. V. Shannon (Am. Mineralogist, vol. 6, pp. 82-83, 1921).

Teallite (?) (SnS.PbS).-Orthorhombic; color gray; hardness D-. HNO, fumes tarnish slowly. $\mathrm{HCl}$ stains black and brings out scratches. $\mathrm{KCN}, \mathrm{FeCl}$ $\mathrm{KOH}$, and $\mathrm{HgCl}_{2}$ negative. Has good cleavage in at least two directions and shows numerous triangular pits. Specimen contains $\mathrm{Pb}$ and $\mathrm{Sn}$, but $\mathrm{Sb}$ is absent Does not check in physical characteristics with teallite. May be some other compound of lead and tin.

Tenorite or melaconite $(\mathrm{CuO})$.- - Monoclinic, color gray; hardness E-. Moder. ately strong anisotropism. $\mathrm{HNO}_{3}$ fumes tarnish; surface slowly stains differer. tially brown; some areas unaffected. $\mathrm{HCl}$ slowly stains differentially brom; some areas unaffected; drop colors green. $\mathrm{KCN}, \mathrm{FeCl}_{3}, \mathrm{KOH}$, and $\mathrm{HgCl}_{2}$ negative Found in the oxidized zone of copper deposits. The massive variety, the more common, is melaconite; and the prismatic variety, tenorite. Melaconite commonly has more or less chemically combined manganese oxide and silica. If it contains more than about 5 per cent of either of these two components the minexl is known as "copper pitch ore."

Hausmannite $\left(\mathrm{Mn}_{3} \mathrm{O}_{4}\right)$. - Hexagonal; color white with a faint tinge of blee; hardness D-. Strongly anisotropic; most specimens show multiple twinning resembling that of plagioclase. $\mathrm{HNO}_{3}$ fumes tarnish; some specimens negative $\mathrm{HCl}$ tarnishes differentially brown in places; some areas negative. $\mathrm{KCN}_{1}$ $\mathrm{FeCl}_{3}, \mathrm{KOH}$, and $\mathrm{HgCl}_{2}$ negative. $\mathrm{H}_{2} \mathrm{O}_{2}$ effervesces slowly without staining surface. Good basal cleavage. Powder and internal reflection deep red. Taks a high polish.

\section{$\mathrm{HNO}_{3}$, $\mathrm{HCl}$ neg., $\mathrm{KCN}, \mathrm{FeCl}_{3}$} ISOTROPIC

КОН

$\mathrm{HgCl}_{2}$

Domeykite $\left(\mathrm{Cu}_{3} \mathrm{As}\right)$.- - Isometric; color creamy gray; hardnes C. $\mathrm{HNO}_{3}$ effervesces and brings out lamellar structure. $\mathrm{HO}$ brings out etch cleavage; some areas, action very slow, reaction doubtful. KCN brings out etch cleavage. $\mathrm{FeCl}_{3}$ quiekly stains iridescent. $\mathrm{KOH}$ and $\mathrm{HgCl}_{2}$ stain iridescent and bring out lamelly structure. Almost sectile but yields powder when scratched. 
Antimony (Sb).-Hexagonal; color silvery white; hardness B. коH neg. Apparently isotropic. $\mathrm{HNO}_{3}$ quickly stains black. $\mathrm{HCl}$ nega$\mathrm{HgCl}_{3}$ tive; fumes sometimes tarnish faintly. $\mathrm{KCN}$ stains light brown. $\mathrm{FeCl}_{3}$ stains black. $\mathrm{KOH}$ negative. $\mathrm{HgCl}_{2}$ quickly stains iridescent. Rhombohedral cleavage. Exhibits triangular pits.

Dyscrasite ( $\left.\mathrm{Ag}_{3} \mathrm{Sb}\right)$.-Isometric; color silvery white; hardness $\mathrm{C}-\mathrm{H} \mathrm{HNO}_{3}$ quickly stains black; with some specimens brings out grating structure. $\mathrm{HCl}$ negative. KCN slowly stains brown; weak. $\mathrm{FeCl}_{3}$ quickly stains iridescent and brings out structure. $\mathrm{KOH}$ negative. $\mathrm{HgCl}_{2}$ quickly stains brown. Almost sectile but yields powder when scratched.

\section{ANISOTROPIC}

Polybasite $\left(8 \mathrm{Ag}_{2} \mathrm{~S}_{2} \mathrm{Sb}_{2} \mathrm{~S}_{3}\right)$.-Monoclinic; color gray; hardness

KOH

$\mathrm{HgCl}_{2}$ $\mathrm{C}-\mathrm{HNO}_{3}$ fumes tarnish. $\mathrm{HCl}$ negative. $\mathrm{KCN}$ quickly blackens. $\mathrm{FeCl}_{3}$ tarnishes iridescent. $\mathrm{KOH}$ slowly tarnishes iridescent; in some specimens negative. $\mathrm{HgCl}_{2}$ quickly blackens. Powder dark ruby-red; if particles are coarse powder appears black. Internal reflection red; not always seen.

Stephanite $\left(5 \mathrm{Ag}_{2} \mathrm{~S}_{2} \mathrm{Sb}_{2} \mathrm{~S}_{3}\right)$.- Orthorhombic; color galena-white; hardness B. $\mathrm{HNO}_{3}$ fumes tarnish. $\mathrm{HCl}$ negative. $\mathrm{KCN}$ quickly blackens. $\mathrm{FeCl}_{3}$ stains slightly light gray, practically negative. $\mathrm{KOH}$ tarnishes iridescent to black. $\mathrm{HgCl}_{2}$ tarnishes iridescent. Best distinguished from polybasite by weak $\mathrm{FeCl}_{3}$ reaction, metallic powder, and lack of internal reflection.

Naumannite $\left(\left(\mathrm{Ag}_{2}, \mathrm{~Pb}\right) \mathrm{Se}\right)$.-Isometric; color gray; hardness $\mathrm{B}$. коH neg. Weak anomalous anisotropism. $\mathrm{HNO}_{3}$ tarnishes iridescent and $\mathrm{HgCl}_{2} \quad$ brings out structure. $\mathrm{HCl}$ negative. $\mathrm{KCN}$ very slowly etches brown; brings out cleavage. $\mathrm{FeCl}_{3}$ instantly tarnishes iridescent. $\mathrm{KOH}$ negative. $\mathrm{HgCl}_{2}$ tarnishes brown to iridescent and brings out structure. Usually shows traces of cubic cleavage. Isomorphous with and usually undistinguishable from aguilarite, $\mathrm{Ag}_{2} \mathrm{Se}$.

Pearcite $\left(8 \mathrm{Ag}_{2} \mathrm{~S} . \mathrm{As}_{2} \mathrm{~S}_{3}\right)$ - - - Monoclinic; color white; hardness C. $\mathrm{HNO}_{3}$ fumes tarnish. $\mathrm{HCl}$ negative. $\mathrm{KCN}$ quickly stains dark. $\mathrm{FeCl}_{3}$ stains iridescent. $\mathrm{KOH}$ negative. $\mathrm{HgCl}_{2}$ stains iridescent. Very thin splinters are dark red at the edges.

Proustite $\left(3 \mathrm{Ag}_{2} \mathrm{~S}_{2} \mathrm{As}_{2} \mathrm{~S}_{3}\right)$. - Hexagonal; color bluish gray; hardness $\mathrm{B}-. \quad \mathrm{HNO}_{3}$ slowly stains dark. $\mathrm{HCl}$ negative. $\mathrm{KCN}$ quickly stains black and brings out scratches. $\mathrm{FeCl}_{3}$ slowly stains black. $\mathrm{KOH}$ negative. $\mathrm{HgCl}_{2}$ stains brown to iridescent. Color in oblique light bright ruby-red, lighter than pyrargyrite. Powder and internal reflection ruby-red.

Eucairite $\left(\mathrm{Cu}_{2} \mathrm{Se} . \mathrm{Ag}_{2} \mathrm{Se}\right)$.-Isometric; color gray, similar to KOH neg. ehalcocite; hardness $\mathrm{A}-$. . Weakly anisotropic. $\mathrm{HNO}_{3}$ fumes $\mathrm{HgCl}_{2}$ neg. tarnish strongly; surface slowly turns dark gray. $\mathrm{HCl}$ negative. $\mathrm{KCN}$ instantly stains black. $\mathrm{FeCl}_{3}$ instantly tarnishes differentially iridescent. $\mathrm{KOH}$ and $\mathrm{HgCl}_{2}$ negative. Associated with clausthalite and klockmannite.

$\mathrm{HNO}_{3}$, $\mathrm{HCl}$ neg., $\mathrm{KCN}, \mathrm{FeCl}_{3}$ neg. ISOTROPIC

KOH

Canfieldite ( $4 \mathrm{Ag}_{2} \mathrm{~S} . \mathrm{SnS}_{2}$, with some germanium).--Isometric; $\mathrm{HgCl}_{2}$ color gray, with pinkish tinge. Most areas isotropic, but some show anomalous anisotropism. $\mathrm{HNO}_{3}$ slowly stains brown; usually required more than one minute to work. $\mathrm{HCl}$ negative. $\mathrm{KCN}$ stains dark gray and brings out structure. $\mathrm{FeCl}_{3}$ deposits scum of ferric chloride, which tends to cling to surface, but reaction should be termed negative. $\mathrm{KOH}$ tarnishes through iridescent to dark gray. $\mathrm{HgCl}_{2}$ instantly tarnishes iridescent. 
Argyrodite $\left(4 \mathrm{Ag}_{2} \mathrm{~S} . \mathrm{GeS}_{2}\right)$.-Tetragonal, pseudo-isometric; color KOH neg. gray, similar to enargite; hardness C. Some specimens isotropic; $\mathrm{HgCl}_{2}$ others anisotropic. $\mathrm{HNO}_{3}$ fumes tarnish faintly; usually negg. tive. $\mathrm{HCl}$ negative. $\mathrm{KCN}$ slowly stains light brown. $\mathrm{FeCl}_{3}$ and $\mathrm{KOH}$ negative. $\mathrm{HgCl}_{2}$ quickly stains iridescent.

Tennantite $\left(5 \mathrm{Cu}_{2} \mathrm{~S} .2(\mathrm{Cu}, \mathrm{Fe}, \mathrm{Zn}) \mathrm{S} .2 \mathrm{As}_{2} \mathrm{~S}_{3}\right)$. - Isometric; color KOH neg. gray; hardness D. $\mathrm{HNO}_{3}$ fumes tarnish. $\mathrm{HCl}$ negative. $\mathbf{H g C l}_{2}$ neg. KCN, some specimens stain slowly light brown, bringing out scratches; most specimens negative. $\mathrm{FeCl}_{3}, \mathrm{KOH}$, and $\mathrm{HgO}_{4}$ negative. Color distinctly greenish when in contact with galena. Powder metallic in most specimens, reddish brown in some.

Tetrahedrite $\left(5 \mathrm{Cu}_{2} \mathrm{~S} .2(\mathrm{Cu}, \mathrm{Fe}, \mathrm{Zn}) \mathrm{S} .2 \mathrm{Sb}_{2} \mathrm{~S}_{3}\right.$.) - Isometric; color gray; hardness D. $\mathrm{HNO}_{3}$ fumes tarnish; some specimens stain iridescent. $\mathrm{HCl}$ negative. $\mathrm{KCN}$, some specimens stain slowly light brown, bringing out scratches; most specimens negative. $\mathrm{FeCl}_{3}, \mathrm{KOH}$, and $\mathrm{HgCl}_{2}$ negative. Powder metallic in most specimens, reddish brown in some.

\section{ANISOTROPIC}

КОН

Canfieldite ( $4 \mathrm{Ag}_{2} \mathrm{~S} . \mathrm{SnS}_{2}$, with some germanium).-Isometric; $\mathrm{HgCl}_{2}$ color gray with pinkish tinge; hardness B. Most areas isotropic, but some show anomalous anisotropism. $\mathrm{HNO}_{3}$ slowly stains brown; usually requires more than one minute to work; weak. $\mathrm{HCl}$ negative. $\mathrm{KCN}$ stains dark and brings out structure. $\mathrm{FeC}_{4}$ deposits coating of forric chloride which tends to cling to surface, but reaction should be termed negative. KOH tarnishes through iridescent to dark gray. $\mathrm{HgCl}_{2}$ instantly tarnishes iridescent.

Polybasite $\left(8 \mathrm{Ag}_{2} \mathrm{~S} \mathrm{Sb}_{2} \mathrm{~S}_{3}\right)$.- Monoclinic; color gray; hardness $\mathrm{C}-$. $\mathrm{HNO}_{1}$ fumes tarnish. $\mathrm{HCl}$ negative. $\mathrm{KCN}$ stains black. $\mathrm{FeCl}_{3}$ usually tarnishes iridescent; some specimens negative. $\mathrm{KOH}$ slowly tarnishes iridescent; some specimens negative. $\mathrm{HgCl}_{2}$ stains brown to iridescent. Powder dark ruby-red; if particles are coarse, powder appears black. Internal reflection red; not always seen.

Proustite $\left(3 \mathrm{Ag}_{2} \mathrm{~S} . \mathrm{As}_{2} \mathrm{~S}_{3}\right)$.-Hexagonal; color bluish gray; hardness $\mathrm{B}$. $\mathrm{HNO}_{3}$ fumes tarnish. $\mathrm{HCl}$ negative. $\mathrm{KCN}$ quickly stains black. $\mathrm{FeCl}_{3}$ negative. $\mathrm{KOH}$ stains black. $\mathrm{HgCl}_{2}$ stains black. Color and internal reflection ruby-red. Indistinguishable from pyrargyrite by color and etch tests.

Pyrargyrite $\left(3 \mathrm{Ag}_{2} \mathrm{~S} \mathrm{Sb}_{2} \mathrm{~S}_{3}\right)$. - Physical properties and etch tests identical with those of proustite.

КОН

$\mathrm{HgCl}_{2}$ neg.

Kermesite $\left(\mathrm{Sb}_{2} \mathrm{~S}_{2} \mathrm{O}\right)$.- Monoclinic; color gray; hardness $\mathrm{B}$. Strong anisotropism. $\mathrm{HNO}_{3}$, some areas tarnish brown; others negative. $\mathrm{HCl}$ negative. $\mathrm{KCN}$ stains slightly light brown; doubtful. $\mathrm{FeCl}_{3}$ negative. $\mathrm{KOH}$ tarnishes and gives yellor coating; this is the most characteristic reaction for the mineral; reaction is identical with that for stibnite. $\mathrm{HgCl}_{2}$ negative. Prismatic. Red powder and red internal reflection (best seen with strong polarized light).

Livingstonite (HgS.2 $\mathrm{Sb}_{2} \mathrm{~S}_{3}$ ).--Orthorhombic; color lead-gray; hardness $\mathrm{C}$. Strong anisotropism. $\mathrm{HNO}_{3}$ slowly stains brown to iridescent. $\mathrm{HCl}$ negative $\mathrm{KCN}$ stains brown and brings out scratches. $\mathrm{FeCl}_{3}$ negative. $\mathrm{KOH}$ instantly darkens, leaving yellow deposit; action highly characteristic, resembling that of stibnite. $\mathrm{HgCl}_{2}$ negative. Powder is red when finely divided.

Miargyrite ( $\left.\mathrm{Ag}_{2} \mathrm{~S}_{\mathrm{S}} \mathrm{Sb}_{2} \mathrm{~S}_{3}\right)$.-Monoclinic; color grayish white; hardness $\mathrm{B}$. Strong anisotropism. $\mathrm{HNO}_{3}$ usually negative; sometimes stains slightly. $\mathrm{HO}$ 
fumes usually tarnish; sometimes negative. KCN stains differentially dark gray. $\mathrm{FeCl}_{3}$ sometimes tarnishes faintly; doubtful. $\mathrm{KOH}$ tarnishes differentially iridescent. $\mathrm{HgCl}_{2}$ negative. Ruby-red powder and internal reflection. For comparison with pyrargyrite see page 91 .

Stibnite $\left(\mathrm{Sb}_{2} \mathrm{~S}_{3}\right)$.- Orthorhombic; color gray; hardness $\mathrm{B}-$. Very strong anisotropism. $\mathrm{HNO}_{3}$ stains differentially iridescent. $\mathrm{HCl}$ fumes sometimes tarnish slightly; usually reaction is negative. KCN brings out scratches and sometimes stains slightly. $\mathrm{FeCl}_{3}$ negative. $\mathrm{KOH}$ instantly tarnishes and deposits a yellow coating; this is the most characteristic etch reaction for stibnite. $\mathrm{HgCl}_{2}$ negative. Prismatic. Hardness and color vary somewhat with orientation, giving mottled appearance; this may mislead one into believing that more than one mineral is present.

K̇lockmannite (CuSe).-Hexagonal; color greenish gray; hardness C. Strong anisotropism; gives four extinctions per revolution. Interference color fiery like covellite but orange rather than red. $\mathrm{HNO}_{3}$ fumes tarnish. $\mathrm{HCl}$ negative; solution turns yellow. $\mathrm{KCN}$ quickly stains black. $\mathrm{FeCl}_{3}$ negative. $\mathrm{KOH}$ slowly stains differentially brown. $\mathrm{HgCl}_{2}$ negative. Mottled in appearance. Close resemblance to covellite indicates that the above formula is correct..$^{45}$ Associated with clausthalite and other selenides.

Argyrodite $\left(4 \mathrm{Ag}_{2} \mathrm{~S} . \mathrm{GeS}_{2}\right)$.-Tetragonal, pseudo-isometric; color, коH neg. gray, like enargite; hardness C. Some specimens isotropic, $\mathrm{HgCl}_{2}$ others weakly anisotropic. $\mathrm{HNO}_{3}$ fumes sometimes tarnish faintly; usually negative. $\mathrm{HCl}$ negative. $\mathrm{KCN}$ stains light brown. $\mathrm{FeCl}_{3}$ and $\mathrm{KOH}$ negative. $\mathrm{HgCl}_{2}$ quickly tarnishes iridescent; the most characteristic reaction.

Chalcostibite $\left(\mathrm{Cu}_{2} \mathrm{~S} \cdot \mathrm{Sb}_{2} \mathrm{~S}_{3}\right)$.- Orthorhombic; color galena-white;

K0H neg. . hardness C. $\mathrm{HNO}_{3}$ fumes tarnish; mineral stains iridescent $\mathrm{HgCl}_{2}$ neg. after long etching. $\mathrm{HCl}$ negative. $\mathrm{KCN}$ practically negative; brings out scratches and stains slightly. $\mathrm{FeCl}_{3}, \mathrm{KOH}$, and

$\mathrm{HgCl}_{2}$ negative. Lighter in color than tetrahedrite. Has well-marked cleavage.

Enargile $\left(\mathrm{Cu}_{2} \mathrm{~S} .4 \mathrm{CuS} . \mathrm{As}_{2} \mathrm{~S}_{3}\right)$.- Orthorhombic; color, two varieties known, one gray, the other pinkish; the pinkish variety is known as luzonite; both varieties may be seen in the same specimen; hardness D-. Strong anisotropism. $\mathrm{HNO}_{3}$ fumes tarnish faintly; sometimes negative. $\mathrm{HCl}$ negative. $\mathrm{KCN}$ stains black and sometimes brings out a parallel structure. $\mathrm{FeCl}_{3}$ and $\mathrm{KOH}$ negative. $\mathrm{HgCl}_{2}$ sometimes stains slightly; doubtful. In many places intergrown with tennantite.

Famatinite $\left(\mathrm{Cu}_{2} \mathrm{~S} .4 \mathrm{CuS} . \mathrm{Sb}_{2} \mathrm{~S}_{3}\right)$.- Orthorhombic; color pinkish gray, similar to pink enargite; hardness D. Strongly anisotropic. $\mathrm{HNO}_{3}$ stains differentially iridescent; fumes tarnish. $\mathrm{HCl}$ negative. $\mathrm{KCN}$ stains differentially dark; action slower than enargite. $\mathrm{FeCl}_{3}, \mathrm{KOH}$, and $\mathrm{HgCl}_{2}$ negative. Prismatic cleavage. Isomorphous with enargite.

Klaprotholite $\left(4 \mathrm{Cu}_{2} \mathrm{~S} \cdot \mathrm{Bi}_{2} \mathrm{~S}_{3}\right)$.- Probably isometric; color gray, like tetrahedrite; in oblique light it has a pinkish-brown tinge; hardness C. Very weak anisotropism, possibly anomalous. $\mathrm{HNO}_{3}$ slowly stains brown; fumes tarnish. $\mathrm{HCl}$ negative. $\mathrm{KCN}$ stains differentially iridescent. $\mathrm{FeCl}_{3}, \mathrm{KOH}$, and $\mathrm{HgCl}_{2}$ negative. Specimen from Butte, Mont., determined by F. B. Laney as klaprotholite. It is intergrown with coarse-grained covellite in mutual-boundary and subgraphic structures.

"See also Ramdohr, Paul, Klockmannite, ein neues naturaliches Kupferselenid: Centralbl. Mineralogie, 1928, Abt. A, No. 7, pp. 225-232.

15122-31-7 


\title{
$\mathrm{HNO}_{3}, \mathrm{HCl}$ neg., $\mathrm{KCN}$ neg., $\mathrm{FeCl}_{3}$
}

\author{
ISOTROPIC
}

Aguilarite $\left(\mathrm{Ag}_{2}(\mathrm{Se}, \mathrm{S})\right)$.-Isometric; color gray; hardness A KOH neg. $\mathrm{HNO}_{3}$ stains slowly brown. $\mathrm{HCl}$ usually negative; fumes some. $\mathbf{H g C l}_{2}$ times tarnish light brown. KCN negative. $\mathrm{FeCl}_{3}$ tarnishes irides. cent. $\mathrm{KOH}$ negative. $\mathrm{HgCl}_{2}$ stains iridescent. Sectile. Rare.

Dyscrasite ( $\left.\mathrm{Ag}_{3} \mathrm{Sb}\right)$.- Isometric; color silvery white; hardness C. HNO, stains differentially iridescent. $\mathrm{HCl}$ slowly tarnishes differentially; some areas negative or doubtful. $\mathrm{KCN}$ negative. $\mathrm{FeCl}_{3}$ tarnishes iridescent. $\mathrm{KOH}$ negative. $\mathrm{HgCl}_{2}$ stains brown. Almost sectile but yields powder when scratched.

Freibergite $\left(5(\mathrm{Cu}, \mathrm{Ag})_{2} \mathrm{~S} .2(\mathrm{Cu}, \mathrm{Fe}) \mathrm{S} .2 \mathrm{Sb}_{2} \mathrm{~S}_{3}\right)$. - Isometric; color very light gray; hardness $\mathrm{D}$. $\mathrm{HNO}_{3}$ slowly stains brown. $\mathrm{HCl}$ and $\mathrm{KCN}$ negative. $\mathrm{FeCl}$, stains iridescent. $\mathrm{KOH}$ negative. $\mathrm{HgCl}_{2}$ leaves spotty residue; almost nega. tive. Specimens examined are from Randsburg, Calif., and Mayo district, Yukon. Both are relatively high in silver.

Petzite $\left((\mathrm{Ag}, \mathrm{Au})_{2} \mathrm{Te}\right)$.- Isometric; color galena-white; hardness $\mathrm{A}$. $\mathrm{HNO}_{3}$ stains black. $\mathrm{HCl}$ and $\mathrm{KCN}$ negative. $\mathrm{FeCl}_{3}$ stains iridescent. $\mathrm{KOH}$ nega. tive. $\mathrm{HgCl}_{2}$ stains brown to iridescent. Usually intergrown with hessite. Petzite is whiter than hessite and has a smoother surface. Hessite has a rough surface and resembles argentite closely. Petzite has a cubic cleavage and exhibits triangular pits like galena, but the cleavage is less perfect than that of galena. Petzite is a much rarer mineral than hessite; most so-called petzites examined by the writer proved to be intergrowths of hessite with calaverite or other minerals.

Coloradoite (HgTe).--Isometric; color pinkish gray; hardness KOH neg. C. $\mathrm{HNO}_{3}$ slowly stains brown; some areas almost negative. $\mathrm{HgCl}_{2}$ neg. $\mathrm{HCl}$ and $\mathrm{KCN}$ negative. $\mathrm{FeCl}_{3}$ stains differentially iridescent. $\mathrm{KOH}$ and $\mathrm{HgCl}_{2}$ negative.

Kallitite (Ni(Sb,Bi)S).--Isometric; color gray; hardness $\mathrm{D} . \mathrm{HNO}_{3}$ tarnishes iridescent. $\mathrm{HCl}$ and $\mathrm{KCN}$ negative. $\mathrm{FeCl}_{3}$ stains faintly, almost negative. $\mathrm{KOH}$ and $\mathrm{HgCl}_{2}$ negative. From Schoenstein, Germany. Very rare.

Ullmanite (NiSbS).--Isometric; color gray; hardness $\mathrm{E}$. $\mathrm{HNO}_{3}$ stains brown to iridescent. $\mathrm{HCl}$ and $\mathrm{KCN}$ negative. $\mathrm{FeCl}_{3}$ stains light brown. $\mathrm{KOH}$ and $\mathrm{HgCl}_{2}$ negative. Has well-defined cubic cleavage and shows triangular pits.

Willyamite ((Co,Ni)SbS).-Isometric; color gray; hardness E. $\mathrm{HNO}_{1}$ tarnishes iridescent; seems to effervesce slightly. $\mathrm{HCl}$ and $\mathrm{KCN}_{3}$ negative. $\mathrm{FeCl}_{3}$ stains slightly; almost negative. $\mathrm{KOH}$ and $\mathrm{HgCl}_{2}$ negative. From Broken Hill, Australia. Rare.

\section{ANISOTROPIC}

KOH

Wittichenite $\left(3 \mathrm{Cu}_{2} \mathrm{~S}_{2} \mathrm{Bi}_{2} \mathrm{~S}_{3}\right)$. - Color gray; hardness $\mathrm{C}-$. $\mathrm{HNO}$

$\mathrm{HgCl}_{2}$ quickly stains iridescent. $\mathrm{HCl}$ and $\mathrm{KCN}$ negative. $\mathrm{FeCl}_{3}$ stains light brown. $\mathrm{KOH}$ stains iridescent. $\mathrm{HgCl}_{2}$ stains slightly, doubtful. Specimen from Colquijirca, Peru; collected by $\mathrm{Dr}$

Waldemar Lindgren and analyzed at the Massachusetts Institute of Technologer. Aguilarite $\left(\mathrm{Ag}_{2}(\mathrm{Se}, \mathrm{S})\right)$.- - Listed as isometric; color gray; hard-

KOH neg. ness A. Strong anisotropism. $\mathrm{HNO}_{3}$ quickly stains through $\mathrm{HgCl}_{2} \quad$ iridescent to black; fumes tarnish. $\mathrm{HCl}$ and $\mathrm{KCN}$ negative $\mathrm{FeCl}_{3}$ quickly stains iridescent. $\mathrm{KOH}$ negative. $\mathrm{HgOh}$ quickly stains iridescent. The strong anisotropism raises the question whethe this mineral is isometric. Resembles argentite but yields powder when scratched

Hessite ( $\left.\mathrm{Ag}_{2} \mathrm{Te}\right)$.- - Isometric; color gray; hardness $\mathrm{A}+$. Most specimens of hessite exhibit anomalous anisotropism. $\mathrm{HNO}_{3}$ stains black. $\mathrm{HCl}$ neg* 
tive; fumes seem to tarnish faintly on some areas. $\mathrm{KCN}$ negative. $\mathrm{FeCl}_{3}$ stains iridescent. $\mathrm{KOH}$ negative. $\mathrm{HgCl}_{2}$ stains brown to iridescent. Resembles argentite but yields powder when scratched.

Aikinite (CuS.2PbS. $\mathrm{Bi}_{2} \mathrm{~S}_{3}$ ).-Orthorhombic; color white; hardкоH neg. ness C. Strong anisotropism. $\mathrm{HNO}_{3}$, most areas effervesce $\mathrm{HgCl}_{2}$ neg. and stain iridescent; some areas stain without effervescence. $\mathrm{HCl}$ and $\mathrm{KCN}$ negative. $\mathrm{FeCl}_{3}$ stains slightly very light brown; action weak and might be considered negative. $\mathrm{KOH}$ and $\mathrm{HgCl}_{2}$ negative.

Alaskaite $\left(\mathrm{Ag}_{2} \mathrm{~S}_{\text {.PbS. }} \mathrm{PBi}_{2} \mathrm{~S}_{3}\right)$. - Orthorhombic; color gray; hardness C. Strong snisotropism. $\mathrm{HNO}_{3}$ slowly stains dark. $\mathrm{HCl}$ fumes tarnish a little; doubtful. $\mathrm{KCN}$ negative. $\mathrm{FeCl}_{3}$ stains iridescent. $\mathrm{KOH}$ and $\mathrm{HgCl}_{2}$ negative. Argentiferous galenobismutite. Prismatic. Rare. Analysis of a specimen from Silverton, Colo., by J. G. Fairchild shows $\mathrm{Pb}$ 15.4, Ag 11.7, Cu 1.6, Bi 55.3, S 16.0.

Sylvanite (( $\left.\mathrm{Au}, \mathrm{Ag}) \mathrm{Te}_{2}\right)$.-Monoclinic; color creamy white; hardness C. Strong anisotropism. $\mathrm{HNO}_{3}$ stains iridescent with doubtful effervescence. $\mathrm{HCl}$ and $\mathrm{KCN}$ negative. $\mathrm{FeCl}_{3}$ sometimes leaves light-yellow stain; usually negative. $\mathrm{KOH}$ and $\mathrm{HgCl}_{2}$ negative.

\section{$\mathrm{HNO}_{3}$, $\mathrm{HCl}$ neg., $\mathrm{KCN}$ neg., $\mathrm{FeCl}_{3}$ neg.}

ISOTROPIC

Carrollite $\left((\mathrm{Co}, \mathrm{Cu})_{3} \mathrm{~S}_{4}\right)$.- Isometric; color creamy white, KOH neg. similar to linnaeite but somewhat lighter; hardness $\mathrm{E}$. $\mathrm{HNO}_{3}$ $\mathrm{HgCl}_{2}$ fumes tarnish iridescent. $\mathrm{HCl}, \mathrm{KCN}, \mathrm{FeCl}_{3}$, and $\mathrm{KOH}$ negative. $\mathrm{HgCl}_{2}$ stains brown to iridescent. Action somewhat slow. Microchemical tests on carefully selected areas indicate that carrollite is a distinct mineral and not a mixture of linnaeite and copper minerals, as has been averred. 4 $^{\prime}$

Linnaeite $\left(\mathrm{Co}_{3} \mathrm{~S}_{4}\right)$.-Isometric; color creamy white; hardness $\mathrm{E}+$. $\mathrm{HNO}_{3}$ fumes tarnish. $\mathrm{HCl}, \mathrm{KCN}, \mathrm{FeCl}_{3}$, and $\mathrm{KOH}$ negative. $\mathrm{HgCl}_{2}$ stains brown differentially; some areas almost negative.

Siegenite $\left((\mathrm{Co}, \mathrm{Ni})_{3} \mathrm{~S}_{4}\right)$.-Isometric; color creamy white; hardness $\mathrm{E}+$. $\mathrm{HNO}_{3}$ fumes tarnish strongly; surface slowly stains differentially brown. $\mathrm{HCl}, \mathrm{KCN}$, $\mathrm{FeCl}_{3}$, and $\mathrm{KOH}$ negative. $\mathrm{HgCl}_{2}$, some specimens stain slightly, others negative. Readily scratched by needle. Specimen from Carroll County, Md., shows good cubic cleavage. Specimens from Mine la Motte, Mo., and Heinrichsiegen, Germany, show no cleavage. Siegenite is more abundant than linnaeite. Indistinguishable from linnaeite except by microchemical tests for $\mathrm{Ni}$ and Co. The color is best seen by viewing the polished section with the unaided eye in oblique light, tilting it so as to get the reflection from a light source, preferably daylight from a north window.

Chalcopyrite $\left(\mathrm{CuFeS}_{2}\right)$.-Tetragonal; color brass-yellow; KOH neg. hardness C. Most specimens are weakly but discernibly $\mathrm{HgCl}_{2}$ neg. anisotropic, but a small proportion are apparently isotropic. $\mathrm{HNO}_{3}$ fumes tarnish; some varieties negative. $\mathrm{HCl}, \mathrm{KCN}$, $\mathrm{FeCl}_{3}, \mathrm{KOH}$, and $\mathrm{HgCl}_{2}$ negative. Aqua regia fumes tarnish differentially and bring out grain structure; area covered by drop usually stains slowly brown, but some areas almost unaffected. Easily scratched by needle, which distinguishes it from pyrite. Gives powder when scratched, in contrast to gold, which is sectile.

Freibergite $\left(5(\mathrm{Cu}, \mathrm{Ag})_{2} \mathrm{~S} .2(\mathrm{Cu}, \mathrm{Fe}) \mathrm{S} .2 \mathrm{Sb}_{2} \mathrm{~S}_{3}\right)$.- -Isometric; color gray; hardness D. $\mathrm{HNO}_{3}$ stains iridescent; on some varieties fumes tarnish, but mineral

\footnotetext{
"See Shannon, E. V., The identity of carrollite with linnaeite: Am. Jour. Sci., 5th ser., vol. 11, pD. 489-493, 1926.
} 
covered by drop not affected. $\mathrm{HCl}, \mathrm{KCN}, \mathrm{FeCl}_{3}, \mathrm{KOH}$, and $\mathrm{HgCl}_{2}$ negative, Silver-bearing tetrahedrite.

Pentlandite ((Ni,Fe)S).-Isometric; color brass-yellow; hardness $\mathrm{D}$ $\mathrm{HNO}_{3}$ fumes tarnish; mineral slowly stains brown. $\mathrm{HCl}, \mathrm{KCN}, \mathrm{FeCl}_{3}, \mathrm{KOH}$ and $\mathrm{HgCl}_{2}$ negative. Imperfect cubic cleavage. Nonmagnetic. Almoso invariably associated with pyrrhotite and chalcopyrite.

Sphalerite $(\mathrm{ZnS})$.- Isometric; color gray; hardness $\mathrm{C}+. \mathrm{HNO}_{3}$ fumes tas. nish. $\mathrm{HCl}, \mathrm{KCN}, \mathrm{FeCl}_{3}, \mathrm{KOH}$, and $\mathrm{HgCl}_{2}$ negative. Aqua regia effervescer and stains dark brown; fumes tarnish. Some specimens show resin-colored internal reflection. Some show triangular pits. Powder white to brown. If proportion of iron is high powder may be metallic.

Stannite $\left.\left(\mathrm{Cu}_{2} \mathrm{~S} \text {.FeS.SnS }\right)_{2}\right)$. - Isometric; color light brownish gray; hardness $\mathrm{D}+$. Most specimens show weak anomalous anistropism; others are isotropic. $\mathrm{HNO}_{1}$ stains iridescent; fumes tarnish. $\mathrm{HCl}, \mathrm{KCN}, \mathrm{FeCl}_{3}, \mathrm{KOH}$, and $\mathrm{HgCl}_{2}$ negative. Usually associated with chalcopyrite.

Tennantite $\left(5 \mathrm{Cu}_{2} \mathrm{~S} .2(\mathrm{Cu}, \mathrm{Fe}, \mathrm{Zn}) \mathrm{S} .2 \mathrm{As}_{2} \mathrm{~S}_{3}\right)$.- Isometric; color gray; tennantite in contact with galena shows distinct greenish tinge; hardness D. HNO, fumes tarnish. $\mathrm{HCl}$ negative. $\mathrm{KCN}$ usually negative; stains light brown and brings out scratches on some specimens. $\mathrm{FeCl}_{3}, \mathrm{KOH}$, and $\mathrm{HgCl}_{2}$ negg. tive. Some specimens yield reddish-brown powder, others black metallic powder,

Tetrahedrite $\left(5 \mathrm{Cu}_{2} \mathrm{~S} .2(\mathrm{Cu}, \mathrm{Fe}, \mathrm{Zn}) \mathrm{S} .2 \mathrm{Sb}_{2} \mathrm{~S}_{3}\right)$. - Isometric; color gray; hardness $\mathrm{D}$. $\mathrm{HNO}_{3}$ fumes usually tarnish; some specimens tarnish iridescent, but usually requires more than one minute for test to work. $\mathrm{HCl}, \mathrm{KCN}, \mathrm{FeCl}_{3}, \mathrm{KOH}$, and $\mathrm{HgCl}_{2}$ negative. Some specimens yield reddish-brown powder; others yield black metallic powder.

Willyamite ((Co, Ni) SbS).-Isometric; color gray; hardness $\mathrm{E}$. $\mathrm{HNO}_{3}$ tarnishes iridescent; seems to effervesce slightly. $\mathrm{HCl}$ and $\mathrm{KCN}$ negative. $\mathrm{FeCl}_{4}$ seems to stain slightly; this reaction is easily overlooked. $\mathrm{KOH}$ and $\mathrm{HgCl}$ negative.

Ullmanite and kallilite.-Reactions like willyamite.

Wurtzite ( $\mathrm{ZnS})$.-Hexagonal; color dark gray; hardness $\mathrm{D}-$. Apparently isotropic. $\mathrm{HNO}_{3}$ fumes tarnish. $\mathrm{HCl}, \mathrm{KCN}, \mathrm{FeCl}_{3}, \mathrm{KOH}$, and $\mathrm{HgCl}_{2}$ negar tive. Aqua regia fumes tarnish; surface stains brown. Occurs most commonly as coatings on sphalerite and other sulphides. In the hand specimen, as in pol. ished section, sphalerite and wurtzite are distinguished with difficulty. The only certain way is to examine crushed fragments microscopically between crossed nicols, using transmitted light. Sphalerite is isotropic, whereas wurzite exhibits strong birefrigence.

\section{ANISOTROPIC}

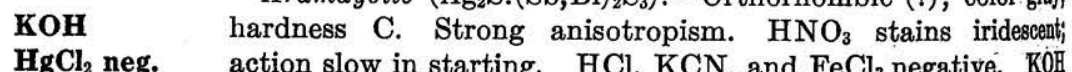

Aramayoite $\left(\mathrm{Ag}_{2} \mathrm{~S}\right.$. $(\mathrm{Sb}, \mathrm{Bi})_{2} \mathrm{~S}_{3}$ ).-Orthorhombic (?); color gray, action slow in starting. $\mathrm{HCl}, \mathrm{KCN}$, and $\mathrm{FeCl}_{3}$ negative. $\mathrm{KOH}$ stains iridescent. $\mathrm{HgCl}_{2}$ negative. Prismatic. Specimen from Chocaya, Bolivia, described by L. J. Spencer (Mineralog. Mag., vol. 21, ppi, 156-162, 1927).

Berthierite ( $\mathrm{FeS} . \mathrm{Sb}_{2} \mathrm{~S}_{3}$ ).-Orthorhombic; color gray; hardness $\mathrm{D}-$. Strong anisotropism. $\mathrm{HNO}_{3}$ tarnishes differentially brown to iridescent. $\mathrm{HCl}$ negative; solution sometimes turns yellow. $\mathrm{KCN}$ and $\mathrm{FeCl}_{3}$ negative. $\mathrm{KOH}$ tarnishes iridescent. $\mathrm{HgCl}_{2}$ negative. Prismatic.

Cylindrite (6PbS. $\left.\mathrm{Sb}_{2} \mathrm{~S}_{3} \cdot 6 \mathrm{SnS}_{2}\right)$. - Tetragonal; color gray; hardness $\mathrm{C}$-. Strong anisotropism. $\mathrm{HNO}_{3}$ slowly stains iridescent. $\mathrm{HCl}, \mathrm{KCN}$, and $\mathrm{FeCl}_{3}$ negative. $\mathrm{KOH}$ slowly stains brown. $\mathrm{HgCl}_{2}$ negative. Prismatic. Basal sections mort or less cylindrical and are made up of concentric shells. 
Franckeite $\left(5 \mathrm{PbS} . \mathrm{Sb}_{2} \mathrm{~S}_{3} \cdot 2 \mathrm{SnS}_{2}\right)$.-Color gray; hardness B. $\mathrm{HNO}_{3}$ stains differentially iridescent; fumes tarnish. $\mathrm{HCl}$ fumes tarnish after long etching; dóubtful. $\mathrm{KCN}$ and $\mathrm{FeCl}_{3}$ negative. $\mathrm{KOH}$, someareas stain slightly, others negative. $\mathrm{HgCl}_{2}$ negative.

Pyrrhotite $\left(\mathrm{FeS}_{1}+\right)$. - Hexagonal; color brownish or pinkish cream; hardness D-. Strong anisotropism. $\mathrm{HNO}_{3}$ slowly stains light brown; fumes tarnish. $\mathrm{HCl}$, solution sometimes turns yellow, but mineral is unaffected. $\mathrm{KCN}$ and $\mathrm{FeCl}_{3}$ negative. $\mathrm{KOH}$ tarnishes iridescent. $\mathrm{HgCl}_{2}$ negative. Distinctly magnetic.

Rathite (3PbS.2 $\left.\mathrm{As}_{2} \mathrm{~S}_{3}\right)$.- Orthorhombic; color galena-white; hardness C. Weak anisotropism. $\mathrm{HNO}_{3}$ effervesces slowly and stains black; effervescence not always observed. $\mathrm{HCl}, \mathrm{KCN}$, and $\mathrm{FeCl}_{3}$ negative. $\mathrm{KOH}$ stains brown. $\mathrm{HgCl}_{2}$ negative. Very rare; only locality reported Allchar, Macedonia.

Stibnite $\left(\mathrm{Sb}_{2} \mathrm{~S}_{3}\right)$.-Orthorhombic; color gray to galena-white; hardness $\mathrm{B}+$. Very strong anisotropism. $\mathrm{HNO}_{3}$ stains differentially iridescent. $\mathrm{HCl}$ fumes usually tarnish slightly on a smooth surface; on most areas negative. KCN on a smooth surface brings out scratches and sometimes stains slightly; this reaction may not be noticeable on a rough surface. $\mathrm{FeCl}_{3}$ negative. $\mathrm{KOH}$ instantly tarnishes and deposits a yellow coating; this is the most characteristic etch reaction for stibnite. $\mathrm{HgCl}_{2}$ negative. Prismatic. Hardness and color vary somewhat with orientation, giving mottled appearance; this may give the impression that more than one mineral is present.

Vrbaite $\left(\mathrm{Tl}_{2} \mathrm{~S} .3(\mathrm{As}, \mathrm{Sb})_{2} \mathrm{~S}_{3}\right)$.-Orthorhombic; color gray; hardness C. Weak anisotropism. $\mathrm{HNO}_{3}$ fumes tarnish. $\mathrm{HCl}, \mathrm{KCN}$, and $\mathrm{FeCl}_{3}$ negative. $\mathrm{KOH}$ instantly stains iridescent and brings out parallel etch cleavage. $\mathrm{HgCl}_{2}$ negative. Red powder and internal reflection. Only locality reported is Allchar, Macedonia. Millerite (NiS).-Hexagonal; color brass-yellow, between color коH neg. of pyrite and that of chalcopyrite; hardness E. Strong aniso$\mathrm{HgCl}_{2}$ tropism. $\mathrm{HNO}_{3}$ slowly. stains brown; fumes tarnish. $\mathrm{HCl}$, $\mathrm{KCN}, \mathrm{FeCl}_{3}$, and $\mathrm{KOH}$ negative. $\mathrm{HgCl}_{2}$ slowly stains brown. Usually but not invariably fibrous in habit.

Aikinite (CuS.2PbS. $\mathrm{Bi}_{2} \mathrm{~S}_{3}$ ).-Orthorhombic; color galena-white; KоH neg. hardness C. Strong anisotropism. $\mathrm{HNO}_{3}$, most areas effervesce $\mathrm{HgCl}_{2}$ neg. and stain iridescent to black; a few areas seem to stain without effervescence. $\mathrm{HCl}, \mathrm{KCN}, \mathrm{FeCl}_{3}, \mathrm{KOH}$, and $\mathrm{HgCl}_{2}$ negative.

Berthonite $\left(2\left(\mathrm{PbCu}_{2}\right) \mathrm{S}_{\mathrm{S}} \mathrm{Sb}_{2} \mathrm{~S}_{3}\right)$.-Color gray, about like tetrahedrite; hardness $\mathrm{C}$. $\mathrm{HNO}_{3}$ fumes tarnish; mineral slowly stains brown to iridescent. Other reagents negative. Massive; no cleavage. From Tunisia. Described by H. Buttgenbach (Soc. géol. Belgique Annales, vol. 46, p. 212, 1923).

Bournonite $\left(\mathrm{Cu}_{2} \mathrm{~S} .2 \mathrm{PbS} . \mathrm{Sb}_{2} \mathrm{~S}_{3}\right)$.- Orthorhombic; color gray, about like tetrahedrite; hardness $\mathrm{C}-\mathrm{H} \mathrm{HNO}_{3}$ fumes tarnish slowly; some specimens tarnish slightly light brown. $\mathrm{HCl}, \mathrm{KCN}, \mathrm{FeCl}_{3}, \mathrm{KOH}$, and $\mathrm{HgCl}_{2}$ negative. Aqua regis effervesces and stains iridescent. Distinguished from tetrahedrite by means of polarized light.

Brongniardite $\left(\mathrm{Ag}_{2} \mathrm{~S} . \mathrm{PbS} . \mathrm{Sb}_{2} \mathrm{~S}_{3}\right)$.-Color galena-white; in contact with galena it has a suggestion of greenish gray; hardness C. Weak anisotropism. $\mathrm{HNO}_{3}$ negative; after long etching slowly stains differentially iridescent. $\mathrm{HCl}, \mathrm{KCN}$, $\mathrm{FeCl}_{3}, \mathrm{KOH}$, and $\mathrm{HgCl}_{2}$ negative. Aqua regia quickly effervesces and stains differentially iridescent. Rare.

Chalcopyrite $\left(\mathrm{CuFeS}_{2}\right)$.-Tetragonal; color brass-yellow; hardness C. Specimens from most localities are weakly anisotropic; those from a few localities, notably Insizwa, South Africa, show fairly strong anisotropism. Chalcopyrite associated with pyrrhotite usually shows multiple twinning in polarized light. $\mathrm{HNO}_{3}$ fumes tarnish; some varieties negative. $\mathrm{HCl}, \mathrm{KCN}, \mathrm{FeCl}_{3}, \mathrm{KOH}$, and 
$\mathrm{HgCl}_{2}$ negative. Aqua regia fumes tarnish differentially and bring out grain structure; area covered by drop usually stains slowly brown, but some areses almost unaffected. Easily scratched by needle, which distinguishes it from pyrite; gives powder when scratched, in contrast to gold, which is sectile.

Chalcostibite $\left(\mathrm{Cu}_{2} \mathrm{~S} \mathrm{Sb}_{2} \mathrm{~S}_{3}\right)$.-Orthorhombic; color white; hardness C. $\mathrm{HNO}_{1}$ fumes tarnish. $\mathrm{HCl}, \mathrm{KCN}, \mathrm{FeCl}_{3}, \mathrm{KOH}$, and $\mathrm{HgCl}_{2}$ negative. Two good cleavages. Distinguished from tetrahedrite by means of polarized light. The only undoubted chalcostibite seen by the author is from Raz-el-Azul, Algerig; other so-called chalcostibites have proved to be tetrahedrite.

Cubanite (CuS.2FeS).-Orthorhombic; color brownish or pinkish cream; harl. ness C. Strong anisotropism. $\mathrm{HNO}_{3}$, some specimens stain light brown; others negative. $\mathrm{HCl}, \mathrm{KCN}, \mathrm{FeCl}_{3}, \mathrm{KOH}$, and $\mathrm{HgCl}_{2}$ negative. Invariably inter. grown with chalcopyrite and usually associated with pyrrhotite. Color almoss identical but a little yellower than that of pyrrhotite. Cubanite is weakly magnetic; less so than pyrrhotite. Cubanite shows no relief with chalcopyrite; pyrrhotite is harder than chalcopyrite and stands in relief above it. Also knom as chalmersite.

Emplectite $\left(\mathrm{Cu}_{2} \mathrm{~S} . \mathrm{Bi}_{2} \mathrm{~S}_{3}\right)$.-Orthorhombic; color creamy gray; hardness C. Strong anisotropism. $\mathrm{HNO}_{3}$, most specimens effervesce and stain light brow; some specimens, however, stain without effervescence, and on some specimens the only effect is a brown halo made by the fumes. $\mathrm{HCl}$ negative; with some speei. mens the drop turns yellow, but the mineral does not stain. $\mathrm{KCN}, \mathrm{FeCl}_{3}, \mathrm{KOH}$, and $\mathrm{HgCl}_{2}$ negative. The color is the most characteristic property of this mineral.

Geocronite (5PbS. $\mathrm{Sb}_{2} \mathrm{~S}_{3}$ ).-Orthorhombic; color galena-white. $\mathrm{HNO}_{3}$ slowly effervesces; mineral turns black; effervescence not always observed. $\mathrm{HCl}, \mathrm{KCN}_{1}$ $\mathrm{FeCl}_{3}, \mathrm{KOH}$, and $\mathrm{HgCl}_{2}$ negative. Prismatic. Rare.

Guanajuatite $\left(\mathrm{Bi}_{2}(\mathrm{Se}, \mathrm{S})_{3}\right)$.-Orthorhombic; color gray; hardness $\mathrm{B}+$. Strong anisotropism. $\mathrm{HNO}_{3}$ tarnishes differentially iridescent. $\mathrm{HCl}, \mathrm{KCN}, \mathrm{FeC}$, $\mathrm{KOH}$, and $\mathrm{HgCl}_{2}$ negative. Prismatic.

Guitermanite ( $3 \mathrm{PbS} . \mathrm{As}_{2} \mathrm{~S}_{3}$ ). - Orthorhombic; color galena-white; hardness $\mathrm{C}_{\text {, }}$ $\mathrm{HNO}_{3}$ fumes tarnish; mineral slowly stains iridescent. $\mathrm{HCl}, \mathrm{KCN}, \mathrm{FeCl}_{3}, \mathrm{KO}$, and $\mathrm{HgCl}_{2}$ negative. Specimen from Zuñi mine, Silverton, Colo., the type locality. Rare.

Jordanite (4PbS. $\left.\mathrm{As}_{2} \mathrm{~S}_{3}\right)$.-Monoclinic; color galena-white; hardness C. HNO, slowly tarnishes gray to brown. $\mathrm{HCl}, \mathrm{KCN}, \mathrm{FeCl}_{3}, \mathrm{KOH}$, and $\mathrm{HgCl}_{2}$ negative Very rare; only localities noted are Binnenthal, Switzerland, and Nagyag Hungary.

Krennerite (( $\left.\mathrm{Au}, \mathrm{Ag}) \mathrm{Te}_{2}\right)$.-Orthorhombic; color creamy white; hardness $\mathrm{C}$. Strong anisotropism. $\mathrm{HNO}_{3}$ slowly effervesces and stains brown; effervescene not always observed. $\mathrm{HCl}, \mathrm{KCN}, \mathrm{FeCl}_{3}, \mathrm{KOH}$, and $\mathrm{HgCl}_{2}$ negative. Pri matic. Distinguished from sylvanite only by external crystal form.

Lorandite $\left(\mathrm{Tl}_{2} \mathrm{~S} . \mathrm{As}_{2} \mathrm{~S}_{3}\right)$.-Monoclinic; color gray; hardness $\mathrm{B}$. $\mathrm{HNO}_{3}$ fumes give permanent tarnish; mineral stains slowly in places. $\mathrm{HCl}, \mathrm{KCN}, \mathrm{FeCl}_{4}$ $\mathrm{KOH}$, and $\mathrm{HgCl}_{2}$ negative. Very rare; only locality reported Allchar, Mace donia. Red powder and internal reflection.

Nagyagite ( $\mathrm{Pb}, \mathrm{Au}, \mathrm{Te}, \mathrm{S}$ (?), sulpho-telluride of $\mathrm{Pb}$ and $\mathrm{Au}$ ).-Orthorhombie; color bluish white; hardness $\mathrm{B}-\mathrm{H} \mathrm{HNO}_{3}$ slowly stains iridescent; fumes some times tarnish. $\mathrm{HCl}, \mathrm{KCN}, \mathrm{FeCl}_{3}, \mathrm{KOH}$, and $\mathrm{HgCl}_{2}$ negative. Habit plats. Plates are flexible and frequently curved like those of molybdenite.

Stannite $\left(\mathrm{Cu}_{2} \mathrm{~S}\right.$.FeS. $\left.\mathrm{SnS}_{2}\right)$. - Isometric; color light brownish gray; hardness $\mathrm{D}+$. Most specimens show weak anomalous anisotropism; others isotropic. $\mathrm{HNO}$ stains iridescent; fumes tarnish. $\mathrm{HCl}, \mathrm{HCN}, \mathrm{FeCl}_{3}, \mathrm{KOH}$, and $\mathrm{HgCl}_{2}$ negative Usually associated with chalcopyrite. 
Sylvanite (( $\left.\mathrm{Au}, \mathrm{Ag}) \mathrm{Te}_{2}\right)$.-Monoclinic; color creamy gray; hardness C. $\mathrm{HNO}_{3}$ stains iridescent with doubtful effervescence. $\mathrm{HCl}$ and $\mathrm{KCN}$ negative. $\mathrm{FeCl}_{3}$, some areas leave light-yellow stain, others negative. $\mathrm{KOH}$ and $\mathrm{HgCl}_{2}$ negative.

\section{$\mathrm{HNO}_{3}$ neg., $\mathrm{HCl}, \mathrm{KCN}, \mathrm{FeCl}_{3}$}

\section{ANISOTROPIC}

Miargyrite $\left(\mathrm{Ag}_{2} \mathrm{~S}_{2} \mathrm{Sb}_{2} \mathrm{~S}_{3}\right)$.-Monoclinic; color gray; hardness $\mathrm{B}$.

KOH Strong anisotropism. $\mathrm{HNO}_{3}$ negative. $\mathrm{HCl}$ fumes tarnish $\mathrm{HgCl}_{2}$ slightly; weak. KCN stains black; brings out multitude of scratches. $\mathrm{FeCl}_{3}$ tarnishes differentially brown; slow. $\mathrm{KOH}$ quickly stains iridescent. $\mathrm{HgCl}_{2}$ slowly tarnishes; some specimens feeble or negative. Powder and internal reflection ruby-red. In contact with miargyrite, pyrargyrite is distinctly bluish. Miargyrite is more strongly anisotropic than pyrargyrite. Miargyrite is almost invariably associated with pyrargyrite. Stephanite $\left(5 \mathrm{Ag}_{2} \mathrm{~S}_{2} \mathrm{Sb}_{2} \mathrm{~S}_{3}\right)$.-Orthorhombic; color gray; hardness B. $\mathrm{HNO}_{3}$ practically negative; after long etching fumes tarnish faintly and mineral seems to stain very light brown; reaction doubtful. $\mathrm{HCl}$ fumes tarnish; halo will not wash off. KCN stains dark gray and brings out scratches. $\mathrm{FeCl}_{3}$ tarnishes. $\mathrm{KOH}$ quickly stains black. $\mathrm{HgCl}_{2}$ quickly stains iridescent. Metallic powder.

\section{$\mathrm{HNO}_{3}$ neg., $\mathrm{HCl}, \mathrm{KCN}, \mathrm{FeCl}_{3}$ neg.}

\section{ANISOTROPIC}

KOH

Stephanite (5 $\left.\mathrm{Ag}_{2} \mathrm{~S}_{2} \mathrm{Sb}_{2} \mathrm{~S}_{3}\right)$.-Orthorhombic; color gray; hard$\mathrm{HgCl}_{2}$ ness B. $\mathrm{HNO}_{3}$ negative. $\mathrm{HCl}$ fumes tarnish; action slow in starting; halo does not wash off. $\mathrm{KCN}$ quickly stains black. $\mathrm{FeCl}_{3}$, most specimens tarnish iridescent; some practically negative, but reagent leaves a scum on the surface which rubs off easily. KOH, some areas stain brown; others unaffected. $\mathrm{HgCl}_{2}$ stains brown to iridescent.

Miargyrite $\left(\mathrm{Ag}_{2} \mathrm{~S}_{2} \mathrm{Sb}_{2} \mathrm{~S}_{3}\right)$.--Monoclinic; color gray; hardness $\mathrm{B}$.

KOH

$\mathrm{HgCl}_{2}$ neg. places negative. $\mathrm{KCN}$ slowly stains brown. $\mathrm{FeCl}_{3}$ negative. $\mathrm{KOH}$ stains iridescent. $\mathrm{HgCl}_{2}$ negative. Powder and internal reflection ruby-red. Usually intergrown with pyrargyrite. Miargyrite has the stronger anisotropism. Pyrargyrite has a distinct bluish tinge when in contact with miargyrite.

\section{$\mathrm{HNO}_{3}$ neg., $\mathrm{HCl}, \mathrm{KCN}$ neg., $\mathrm{FeCl}_{3}$ neg.}

\section{ISOTROPIC}

KOH neg.

Hauerite $\left(\mathrm{MnS}_{2}\right)$.-Isometric; color gray; hardness E. $\mathrm{HNO}_{3}$ $\mathrm{HgCl}_{2}$ neg. fumes tarnish slowly; some areas negative. $\mathrm{HCl}$ solution turns brown; mineral stains light brown. $\mathrm{KCN}, \mathrm{FeCl}_{3}, \mathrm{KOH}$, and $\mathrm{HgCl}_{2}$ negative. Triangular pits indicate cubic cleavage. Red powder.

Magnetite $\left(\mathrm{Fe}_{3} \mathrm{O}_{4}\right)$.- Isometric; color gray; hardness $\mathrm{F}$. $\mathrm{HNO}_{3}$ negative. $\mathrm{HCl}$ on smooth surfaces larger than drop, fumes tarnish; solution turns yellow. $\mathrm{KCN}, \mathrm{FeCl}_{3}, \mathrm{KOH}$, and $\mathrm{HgCl}_{2}$ negative. Aqua regia solution turns yellow. Black powder when scratched. Powder is magnetic. (See description of chromite, p. 102.) Magnetite has the property of yielding somewhat to pressure. A needle drawn across a smooth surface will often leave a distinct mark. This is not a true scratch, as the sides of the mark are unbroken. It will be found difficult to push the point of the needle diagonally against the surface and thus obtain a powder. Magnetite is properly a "hard" mineral but is placed in the "soft" group to obviate the difficulty caused by its anomalous behavior. 
ANISOTROPIC

KOH neg. Delafossite $\left(\mathrm{CuFeO}_{2}\right)$.-Color gray; hardness E. Strong aniso. $\mathbf{H g C l}_{2}$ neg. tropism; shows radial structure. $\mathrm{HNO}_{3}$ negative. $\mathrm{HCl}$ etches $\mathbf{H g C l}_{2}$ neg. differentially black; drop turns yellow. $\mathrm{KCN}, \mathrm{FeCl}_{3}, \mathrm{KOH}$, and $\mathrm{HgCl}_{2}$ negative. Black powder, showing tiny crystalline plates. Very rare.

Freieslebenite $\left(3 \mathrm{Ag}_{2} \mathrm{~S} .4 \mathrm{PbS} .3 \mathrm{Sb}_{2} \mathrm{~S}_{3}\right)$. - Monoclinic; color gray, like tetrahedrite; hardness B. Strong anisotropism. $\mathrm{HNO}_{3}$ negative. $\mathrm{HCl}$ fumes tarnish; halo does not wash off. $\mathrm{KCN}, \mathrm{FeCl}_{3}, \mathrm{KOH}$, and $\mathrm{HgCl}_{2}$ negative. Prismatic. Very rare. Specimen is from Hiendelencia, Spain.

Hausmannite $\left(\mathrm{Mn}_{3} \mathrm{O}_{4}\right)$. - Hexagonal; color white with a faint tinge of blue; hardness D-. Strongly anisotropic; most specimens show multiple twinning resembling that of plagioclase. $\mathrm{HNO}_{3}$ fumes tarnish; some specimens negative. $\mathrm{HCl}$ tarnishes differentially light brown; some areas negative. $\mathrm{KCN}, \mathrm{FeCl}_{4}$, $\mathrm{KOH}$, and $\mathrm{HgCl}_{2}$ negative. $\mathrm{H}_{2} \mathrm{O}_{2}$ effervesces slowly without staining surface. Good basal cleavage. Powder and internal reflection deep red. Takes a high polish.

Manganite $\left(\mathrm{Mn}_{2} \mathrm{O}_{3}\right)$.-Orthorhombic; color gray; hardness E+. Strongly anisotropic; gives four extinctions per revolution. $\mathrm{HNO}_{3}$ negative. $\mathrm{HCl}$ fumes tarnish; surface stains light brown. $\mathrm{KCN}, \mathrm{FeCl}_{3}, \mathrm{KOH}$, and $\mathrm{HgCl}_{2}$ negative. $\mathrm{H}_{2} \mathrm{O}_{2}$ effervesces slowly without staining surface. Prismatic. Gives brown powder.

Teallite (SnS.PbS).-Color galena-white; hardness C. $\mathrm{HNO}_{3}$ negative. $\mathrm{HCl}$ quickly tarnishes gray to brown. $\mathrm{KCN}, \mathrm{FeCl}_{3}, \mathrm{KOH}$, and $\mathrm{HgCl}_{2}$ negative. Perfect basal cleavage. Plates are flexible and curved like those of molybdenite.

\section{$\mathrm{HNO}_{3}$ neg., $\mathrm{HCl}$ neg., $\mathrm{KCN}, \mathrm{FeCl}_{3}$ ISOTROPIC}

Argentite $\left(\mathrm{Ag}_{2} \mathrm{~S}\right)$.--Isometric; color gray; hardness A. Some KOH neg. specimens are isotropic; others show anomalous anisotropism. $\mathrm{HgCl}_{2} \mathrm{HNO}_{3}$ and $\mathrm{HCl}$ fumes tarnish most specimens, but some are negative. KCN stains differentially black and brings out strue. ture. $\mathrm{FeCl}_{3}$ quickly stains black. $\mathrm{KOH}$ negative. $\mathrm{HgCl}_{2}$ quickly stains black. Very sectile; does not yield powder.

\section{ANISOTROPIC}

Argentite.-See above.

$\mathrm{HNO}_{3}$ neg., $\mathrm{HCl}$ neg., $\mathrm{KCN}, \mathrm{FeCl}_{3}$ neg. ISOTROPIC

KOH

Cerargyrite ( $\mathrm{AgCl})$.-Isometric; color dark gray; hardness A.

$\mathbf{H g C l}_{2}$ neg. $\mathrm{FeCl}_{3}$ negative. $\mathrm{KOH}$ stains brown. $\mathrm{HgCl}_{2}$ negative. Waxy quality of cerargyrite. yellow in oblique light. Very sectile; the most characteristic

Argyrodite $\left(4 \mathrm{Ag}_{2} \mathrm{~S} . \mathrm{GeS}_{2}\right)$.-Tetragonal, pseudo-isometric; color KOH neg. gray; hardness C. Some specimens isotropic, others weakly $\mathrm{HgCl}_{2}$ anisotropic. $\mathrm{HNO}_{3}$ and $\mathrm{HCl}$ negative. $\mathrm{KCN}$ stains slowly dark and brings out scratches. $\mathrm{FeCl}_{3}$ doubtful or negative. $\mathrm{KOH}$ negative. $\mathrm{HgCl}_{2}$ tarnishes iridescent.

Gold (Au).-Isometric; hardness $\mathrm{B}$. $\mathrm{HNO}_{3}$ and $\mathrm{HCl}$ negative.

KOH neg. $\mathrm{KCN}$ etches black, giving roughened surface. $\mathrm{FeCl}_{3}, \mathrm{KOH}$, and $\mathbf{H g C l}_{2}$ neg. $\mathrm{HgCl}_{2}$ negative. Very sectile.

Sulvanite $\left(\mathrm{Cu}_{3} \mathrm{VS}_{4}\right)$.-Isometric; color galena-white; hardness

C. $\mathrm{HNO}_{3}$ and $\mathrm{HCl}$ negative. $\mathrm{KCN}$ stains slightly and brings out scratches $\mathrm{FeCl}_{3}, \mathrm{KOH}$, and $\mathrm{HgCl}_{2}$ negative. Cubic cleavage, not prominent. Very rare. Specimen from Burra Burra, South Australia. 
Tiemannite (HgSe).-Isometric; color gray; hardness B. $\mathrm{HNO}_{3}$ and $\mathrm{HCl}$ negative. $\mathrm{KCN}$, some areas tarnish iridescent; others negative. $\mathrm{FeCl}_{3}, \mathrm{KOH}$, and $\mathrm{HgCl}_{2}$ negative. Aqua regia effervesces and stains iridescent.

ANISOTROPIC

Pyrargyrite $\left(3 \mathrm{Ag}_{2} \mathrm{~S}_{2} \mathrm{Sb}_{2} \mathrm{~S}_{3}\right)$.- - Hexagonal; color bluish gray; KOH hardness C. $\mathrm{HNO}_{3}$ fumes tarnish slightly on some areas; others $\mathrm{HgCl}_{2}$ negative. $\mathrm{HCl}$ negative. $\mathrm{KCN}$ stains differentially gray to black. $\mathrm{FeCl}_{3}$ negative; some areas seem to tarnish slightly. $\mathrm{KOH}$ instantly tarnishes iridescent. $\mathrm{HgCl}_{2}$ stains brown. Ruby-red powder and internal reflection. (See description of miargyrite, p. 91.)

Livingstonite ( $\left.\mathrm{HgS} .2 \mathrm{Sb}_{2} \mathrm{~S}_{3}\right)$.- Orthorhombic; color gray; hard-

коH ness C. Strong anisotropism. $\mathrm{HNO}_{3}$, some specimens stain $\mathrm{HgCl}_{2}$ neg. iridescent; others negative. $\mathrm{HCl}$ negative. $\mathrm{KCN}$, some specimens stain brown; others negative. $\mathrm{FeCl}_{3}$ negative. $\mathrm{KOH}$ instantly darkens, leaving yellow deposit; reaction highly characteristic and resembles that of stibnite. $\mathrm{HgCl}_{2}$ negative. Aqua regia effervesces and stains iridescent. Powder red when finely divided. Prismatic. Rare.

Miargyrite $\left(\mathrm{Ag}_{2} \mathrm{~S}_{2} \mathrm{Sb}_{2} \mathrm{~S}_{3}\right)$.- Monoclinic; color gray; hardness B. Strong anisotropism. $\mathrm{HNO}_{3}$ and $\mathrm{HCl}$ negative. $\mathrm{KCN}$ stains differentially black and brings out fine scratches. $\mathrm{FeCl}_{3}$ negative. $\mathrm{KOH}$ stains dark. $\mathrm{HgCl}_{2}$ negative. Powder and internal reflection ruby-red. Usually intergrown with pyrargyrite. Miargyrite has the stronger anisotropism. Pyrargyrite has a distinct bluish tinge when in contact with miargyrite.

Orpiment $\left(\mathrm{As}_{2} \mathrm{~S}_{3}\right)$. - Monoclinic; color gray in vertically incident light, yellow in oblique light; hardness $\mathrm{B}$. Strong anisotropism. $\mathrm{HNO}_{3}$ and $\mathrm{HCl}$ negative. KCN etches and brings out structure. $\mathrm{FeCl}_{3}$ negative. $\mathrm{KOH}$ instantly stains dark brown. $\mathrm{HgCl}_{2}$ deposits sediment but does not etch surface. Yellow powder and internal reflection.

Argyrodite $\left(4 \mathrm{Ag}_{2} \mathrm{~S} . \mathrm{GeS}_{2}\right)$.--Isometric; color gray, similar to KOH neg. enargite; hardness C. Some specimens isotropic; others weakly $\mathrm{HgCl}_{2}$ anisotropic. $\mathrm{HNO}_{3}$, sometimes fumes tarnish faintly; usually negative. $\mathrm{HCl}$ negative. $\mathrm{KCN}$ stains light brown. $\mathrm{FeCl}_{3}$ doubtful or negative. $\mathrm{KOH}$ negative. $\mathrm{HgCl}_{2}$ quickly stains iridescent; the most characteristic etch test.

Covellite (CuS).-Hexagonal; color indigo-blue; hardness $\mathrm{B}+$. коH neg. Strong anisotropism; grains range in color from blue to fiery red $\mathrm{HgCl}_{2}$ neg. between crossed nicols. $\mathrm{HNO}_{3}$ and $\mathrm{HCl}$ negative. $\mathrm{KCN}$ stains black. $\mathrm{FeCl}_{3}, \mathrm{KOH}$, and $\mathrm{HgCl}_{2}$ negative.

Enargite $\left(3 \mathrm{Cu}_{2} \mathrm{~S} .4 \mathrm{CuS} . \mathrm{As}_{2} \mathrm{~S}_{3}\right)$.- Orthorhombic; color, two varieties known, one gray, the other pinkish; the pinkish variety is known as luzonite; both varieties may be seen in the same specimen; hardness $\mathrm{D}-$. Strong anisotropism. $\mathrm{HNO}_{3}$ fumes tarnish faintly; sometimes negative. $\mathrm{HCl}$ negative. $\mathrm{KCN}$ stains black and sometimes brings out a parallel structure. $\mathrm{FeCl}_{3}$ and $\mathrm{KOH}$ negative. $\mathrm{HgCl}_{2}$ sometimes stains slightly; doubtful. Commonly intergrown with tennantite.

\section{$\mathrm{HNO}_{3}$ neg., $\mathrm{HCl}$ neg., $\mathrm{KCN}$ neg., $\mathrm{FeCl}_{3}$}

\section{ISOTROPIC}

Coloradoite (HgTe).-Isometric; color gray with a distinct $\mathrm{KOH}$ neg. tinge of pink; hardness $\mathrm{C}-\mathrm{H} \mathrm{HNO}_{3}$, some areas stain slowly $\mathrm{HgCl}_{2}$ neg. light brown to iridescent; other areas negative. $\mathrm{HCl}$ and $\mathrm{KCN}$ $\mathrm{HgCl}_{2}$ negative. negative. $\mathrm{FeCl}_{3}$ stains differentially iridescent. $\mathrm{KOH}$ and 
ANISOTROPIC

кон

$\mathrm{HgCl}_{2}$ neg.

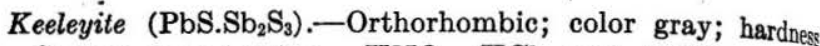
C. Strong anisotropism. $\mathrm{HNO}_{3}, \mathrm{HCl}$, and $\mathrm{KCN}$ negative. $\mathrm{FeCl}_{3}$ slowly tarnishes differentially. $\mathrm{KOH}$ stains black; brings out scratches. $\mathrm{HgCl}_{2}$ negative. Prismatic. Specimen from Oruro, Bolivia. Has same composition as zinkenite but different color and etch reactions. ${ }^{47}$

Tungstenite $\left(\mathrm{WS}_{2}\right)$.-Hexagonal; color gray; hardness A. KOH neg. Strong anisotropism; gives four extinctions per revolution. $\mathrm{HgCl}_{2}$ $\mathrm{HNO}_{3}, \mathrm{HCl}$, and $\mathrm{KCN}$ negative. $\mathrm{FeCl}_{3}$ stains differentially light brown; some areas affected. $\mathrm{KOH}$ negative. $\mathrm{HgCl}$ ? stains iridescent in places; doubtful. Easily scratched by thumb nail. Appears as a steel-blue smear along tiny fracture planes. Very fine grained, porous, and crumbly; polishes with difficulty. Resembles molybdenite but distinguished from it by its fineness of grain. Rare; reported only from Cottonwood, Utal. Analyzed and described by R. C. Wells (Washington Acad. Sci. Jour., vol. ?, pp. 596-599, 1917).

\section{$\mathrm{HNO}_{3}$ neg., $\mathrm{HCl}$ neg., $\mathrm{KCN}$ neg., $\mathrm{FeCl}_{3}$ neg., aqua regia} ISOTROPIC

Chalcopyrite $\left(\mathrm{CuFeS}_{2}\right)$.- Tetragonal; color brass-yellow; hard.

KOH neg. $\mathrm{HgCl}_{2}$ neg. ness C. Most specimens are weakly but discernibly anisotropic; a small proportion are apparently isotropic. $\mathrm{HNO}_{3}$ fumes tarnish; some specimens negative. $\mathrm{HCl}, \mathrm{KCN}, \mathrm{FeCl}_{3}, \mathrm{KOH}$, and $\mathrm{HgCl}_{2}$ negative. Aqua regia fumes tarnish differentially and bring out grain structure; area covered by drop usually stains slowly brown, but some areas unaffected. Easily scratched by needle, which distinguishes it from pyrite. Gives powder when scratched, in contrast to gold, which is sectile.

Germanite $\left(\mathrm{Cu}_{\mathbf{x}} \mathrm{GeS}_{\mathbf{y}}\right)$.- Isometric; color pinkish gray, about the same as pink enargite; hardness C. $\mathrm{HNO}_{3}, \mathrm{KCl}, \mathrm{KCN}, \mathrm{FeCl}_{3}, \mathrm{KOH}$, and $\mathrm{HgCl}_{2}$ negative. Aqua regia slowly stains light brown. From Tsumeb, Southwest Africa; very rare.

Sphalerite $(\mathrm{ZnS}) .-$ Isometric; color gray; hardness C+. $\mathrm{HNO}_{3}$ fumes usually tarnish; mineral stains light brown after long etching; sometimes negative. $\mathrm{HCl}, \mathbf{K C N}, \mathrm{FeCl}_{3}, \mathrm{KOH}$, and $\mathrm{HgCl}_{2}$ negative. Aqua regia effervesces and stains dark brown; fumes tarnish. Some specimens show resin-colored internal reflection. Dodecahedral cleavage; some specimens show triangular pits. Powder white to brown. If proportion of iron is high, powder may be metallic.

Tetrahedrite $\left(5 \mathrm{Cu}_{2} \mathrm{~S} .2(\mathrm{Cu}, \mathrm{Fe}, \mathrm{Zn}) \mathrm{S} .2 \mathrm{Sb}_{2} \mathrm{~S}_{3}\right)$. - Isometric; color gray; hardness D. $\mathrm{HNO}_{3}, \mathrm{HCl}, \mathrm{KCN}, \mathrm{FeCl}_{3}, \mathrm{KOH}$, and $\mathrm{HgCl}_{2}$ negative. Aqua regia fumes tarnish. Some specimens yield reddish-brown powder; others black metallic powder.

Tiemannite (HgSe).-Isometric; color galena-white; hardness $\mathrm{B} . \mathrm{HNO}_{3}, \mathrm{HCl}$, $\mathbf{K C N}, \mathrm{FeCl}_{3}, \mathbf{K O H}$, and $\mathrm{HgCl}_{2}$ negative. Aqua regia instantly effervesces and stains irideseent.

\section{ANISOTROPIC} KOH similar to tetrahedrite; hardness C. Strong anisotropism.
$\mathbf{H g C l}$

Andorite ( $\left.\mathrm{Ag}_{2} \mathrm{~S} .2 \mathrm{PbS} .3 \mathrm{Sb}_{2} \mathrm{~S}_{3}\right)$.-Orthorhombic; color gray, $\mathrm{HNO}_{3}$ fumes usually tarnish but sometimes negative. $\mathrm{HCl}$ negative; fumes sometimes tarnish faintly. $\mathrm{KCN}$ and $\mathrm{FeCl}_{4}$ negative. $\mathrm{KOH}$ slowly stains brown. $\mathrm{HgCl}_{2}$ negative. Aqua regia effervesess

7 See Shannon, E. V., and Short, M. N., A reexamination of the lead sulphosalt keeleyite from Bolivis, Am. Mineralogist, vol. 12, pp. 405-408, 1927; discussion, by E. T. Wherry, idem, vol. 13, pp. 29-30,198. 
vigorously and stains black. Almost invariably associated with stannite. Prismatic.

Berthierite $\left(\mathrm{FeS} . \mathrm{Sb}_{2} \mathrm{~S}_{3}\right)$.-Orthorhombic; color gray; hardness $\mathrm{D}-\mathrm{H} \mathrm{HNO}_{3}$ slowly stains iridescent; usually takes more than a minute before effect begins to show. $\mathrm{HCl}, \mathrm{KCN}$, and $\mathrm{FeCl}_{3}$ negative. $\mathrm{KOH}$ stains iridescent. $\mathrm{HgCl}_{2}$ negative. Aqua regia effervesces and stains light brown. Prismatic.

Kermesite $\left(\mathrm{Sb}_{2} \mathrm{~S}_{2} \mathrm{O}\right)$.-Monoclinic; color bluish gray; hardness B. Strong snisotropism. $\mathrm{HNO}_{3}, \mathrm{HCl}, \mathrm{KCN}$, and $\mathrm{FeCl}_{3}$ negative. $\mathrm{KOH}$ deposits yellow coating; this is the most characteristic etch reaction; resembles that of stibnite. $\mathrm{HgCl}_{2}$ negative. Aqua regia stains differentially iridescent. Reddish-brown powder. . Ruby-red internal reflection with strong polarized light. Habit fibrous.

Livingstonite ( $\mathrm{HgS} .2 \mathrm{Sb}_{2} \mathrm{~S}_{3}$ ).-Orthorhombic; color gray; hardness C. $\mathrm{HNO}_{3}$, some specimens tarnish iridescent; others negative. $\mathrm{HCl}$ negative. $\mathrm{KCN}$, some specimens tarnish brown; others negative. $\mathrm{FeCl}_{3}$ negative. $\mathrm{KOH}$ instantly darkens, leaving yellow deposit; reaction highly characteristic and resembles that of stibnite. $\mathrm{HgCl}_{2}$ negative. Aqua regia effervesces and stains iridescent. Prismatic. Rare. Red powder.

Bournonite ( $\left.\mathrm{Cu}_{2} \mathrm{~S} .2 \mathrm{PbS} . \mathrm{Sb}_{2} \mathrm{~S}_{3}\right)$.-Orthorhombic; color gray, KOH neg. about the same as tetrahedrite; hardness $\mathrm{C}-\mathrm{H} \mathrm{HNO}_{3}$, usually $\mathrm{HgCl}_{2}$ neg. fumes tarnish, some specimens negative. $\mathrm{HCl}, \mathrm{KCN}, \mathrm{FeCl}_{3}$, $\mathrm{KOH}$, and $\mathrm{HgCl}_{2}$ negative. Aqua regia quickly effervesces and stains differentially iridescent.

Brongniardite ( $\mathrm{Ag}_{2} \mathrm{~S} . \mathrm{PbS} . \mathrm{Sb}_{2} \mathrm{~S}_{3}$ ).-Color galena-white; in contact with galena has a suggestion of greenish gray; hardness C. Weak anisotropism. $\mathrm{HNO}_{3}$ negative; after long etching slowly stains differentially iridescent. $\mathrm{HCl}, \mathrm{KCN}$, $\mathrm{FeCl}_{3}, \mathrm{KOH}$, and $\mathrm{HgCl}_{2}$ negative. Aqua regia quickly effervesces and stains differentially iridescent.

Chalcophanite ((Mn, $\left.\mathrm{Zn}) 0.2 \mathrm{MnO}_{2} \cdot 2 \mathrm{H}_{2} \mathrm{O}\right)$.- Orthorhombic; color gray. Strongly anisotropic; gives four extinctions per revolution. $\mathrm{HNO}_{3}$ negative. $\mathrm{HCl}$, drop colors brown; surface does not stain. $\mathrm{KCN}, \mathrm{FeCl}_{3}, \mathrm{KOH}$, and $\mathrm{HgCl}_{2}$ negative. $\mathrm{H}_{2} \mathrm{O}_{2}$ effervesces vigorously, but surface does not stain. Aqua regia, drop colors brown; surface stains differentially light brown. Prismatic. Occurs at Franklin, N. J., associated with franklinite and other zinc minerals.

Chalcopyrite $\left(\mathrm{CuFeS}_{2}\right)$.- Tetragonal; color brass-yellow; hardness C. Specimens from most localities are weakly anisotropic; those from a few localities, notably Insizwa, South Africa, show fairly strong anisotropism. Chalcopyrite associated with pyrrhotite usually shows multiple twinning in polarized light. $\mathrm{HNO}_{3}$ fumes tarnish; some varieties negative. $\mathrm{HCl}, \mathrm{KCN}, \mathrm{FeCl}_{3}, \mathrm{KOH}$, and $\mathrm{HgCl}_{2}$ negative. Aqua regia fumes tarnish differentially and bring out grain structure; area covered by drop usually stains slowly brown, but some areas almost unaffected. Easily scratched by needle, which distinguishes it from pyrite; gives powder when scratched, in contrast to gold, which is sectile.

Cubanite (CuS.2FeS).--Orthorhombic; color brownish or pinkish cream; hardness C. Strong anisotropism. $\mathrm{HNO}_{3}$, some specimens stain light brown; others negative. $\mathrm{HCl}, \mathrm{KCN}, \mathrm{FeCl}_{3}, \mathrm{KOH}$, and $\mathrm{HgCl}_{2}$ negative. Invariably intergrown with chalcopyrite and usually associated with pyrrhotite. Color almost identical but a little yellower than that of pyrrhotite. Cubanite is weakly magnetic; less so than pyrrhotite. Cubanite shows no relief with chalcopyrite; pyrrhotite is harder than chalcopyrite and stands in relief above it. Also known as chalmersite.

Cinnabar (HgS).-Hexagonal; color bluish gray; hardness $\mathrm{B} . \mathrm{HNO}_{3}, \mathrm{HCl}$, $\mathrm{KCN}, \mathrm{FeCl}_{3}, \mathrm{KOH}$, and $\mathrm{HgCl}_{2}$ negative. Aqua regia stains differentially iridescent. Red powder and internal reflection. 
Metacinnabarite (HgS).-Isometric; color gray, black in oblique light; hardness B. Usually shows multiple twinning. $\mathrm{HNO}_{3}, \mathrm{HCl}, \mathrm{KCN}, \mathrm{FeCl}_{3}, \mathrm{KOH}$, and $\mathrm{HgCl}_{2}$ negative. Aqua regia effervesces and turns black. Almost invariably intergrown with cinnabar. In polished sections metacinnabarite has a smooth surface and gives a black powder, whereas cinnabar has a rougher surface and gives red powder and internal reflection.

Molybdenite $\left(\mathrm{MoS}_{2}\right)$.- - Hexagonal; color galena-white; hardness $\mathrm{B} \pm$. Strong anisotropism. Basal sections are isotropic. Prismatic sections give nearly paral. lel extinction and four extinctions per revolution. $\mathrm{HNO}_{3}, \mathrm{KCN}, \mathrm{FeCl}_{3}, \mathrm{KOH}$, and $\mathrm{HgCl}_{2}$ negative. Aqua regia slowly effervesces and darkens. Some areas negative. Perfect basal cleavage; plates tend to be more or less curved.

Nagyagite ( $\mathrm{Pb}, \mathrm{Au}, \mathrm{Te}, \mathrm{S}$ ).-Orthorhombic; color gray; hardness $\mathrm{B}$. HNO, slowly stains iridescent; practically negative. $\mathrm{HCl}, \mathrm{KCN}, \mathrm{FeCl}_{3}, \mathrm{KOH}$, and $\mathrm{HgCl}_{2}$ negative. Aqua regia stains differentially iridescent. Habit platy; plates are flexible like those of molybdenite. From Nagyag, Hungary., Formula doubtful.

$\mathrm{HNO}_{3}$ neg., $\mathrm{HCl}$ neg., $\mathrm{KCN}$ neg., $\mathrm{FeCl}_{3}$ neg., aqua regia neg.

\section{ISOTROPIC}

Realgar (AsS).-Monoclinic; hardness B. Apparently isoKOH tropic; the mineral is fairly transparent and most of the incident $\mathrm{HgCl}_{2}$ neg. light is absorbed. $\mathrm{HNO}_{3}, \mathrm{HCl}, \mathrm{KCN}$, and $\mathrm{FeCl}_{3}$ negative. $\mathrm{KOH}$ instantly stains black. $\mathrm{HgCl}_{2}$ and aqua regia negative. Red internal reflection and powder.

Magnetite $\left(\mathrm{Fe}_{3} \mathrm{O}_{4}\right)$. - Isometric; color gray; hardness $\mathrm{F}$. $\mathrm{HNO}_{3}$ KOH neg. negative. $\mathrm{HCl}$, on smooth surfaces larger than drop, fumes $\mathrm{HgCl}_{2}$ neg. tarnish; solution turns yellow. $\mathrm{KCN}, \mathrm{FeCl}_{3}, \mathrm{KOH}$, and $\mathrm{HgCl}_{9}$ negative. Aqua regia solution turns yellow. Black powder when scratched. Powder is magnetic. (See description of chromite, p. 102.) Magnetite has the property of yielding somewhat to pressure. A needle drawn across a smooth surface will of ten leave a distinct mark. This is not a true scratch, as the sides of the mark are unbroken. It will be found difficult to push the point of the needle diagonally against the surface and thus obtain a powder. Magnetite is properly a "hard" mineral but is placed in the "soft" group as well, in order to obviate the difficulty caused by its anomalous behavior.

\section{ANISOTROPIC}

Molybdenite $\left(\mathrm{MoS}_{2}\right)$.-Hexagonal; color galena-white; hardness $\mathrm{B} \pm$. Strong anisotropism. Basal sections are isotropic; prismatic sections give nearly parallel extinction and four extinctions per revolution. Negative to all reagents. Perfect basal cleavage. Plates tend to be more or less curved.

Pyrolusite $\left(\mathrm{MnO}_{2}\right)$.- Orthorhombic; color gray, almost white, similar to hematite; hardness D to E. Strongly anisotropic. Gives four extinctions per revolution. $\mathrm{HNO}_{3}$ negative. $\mathrm{HCl}$, drop colors brown, but surface does not stain. $\mathrm{KCN}, \mathrm{FeCl}_{2}, \mathrm{KOH}$, and $\mathrm{HgCl}_{2}$ negative. Aqua regia same as $\mathrm{HCl}$. $\mathrm{H}_{2} \mathrm{O}_{21}$ drop effervesces vigorously, but surface does not stain. $\mathrm{H}_{2} \mathrm{O}_{2}+\mathrm{H}_{2} \mathrm{SO}_{4}$, surface stains black. Gives black powder. Hand specimen sooty black. Prismatic; perfect prismatic cleavage. The apparently amorphous variety is seen to be a mat of tiny interlocking needles. Some specimens soil fingers. 
HARD MINERALS

\section{$\mathrm{HNO}_{3}$ eff., $\mathrm{HCl}$ neg., $\mathrm{KCN}$ neg., $\mathrm{FeCl}_{3}$}

ISOTROPIC

Chloanthite-smaltite ((Ni,Co) $\left.\mathrm{As}_{2}\right)$.- - Isometric; color gray; hardKOH neg. $\mathrm{HgCl}_{2}$ ness $\mathrm{F} . \mathrm{HNO}_{3}$ effervesces vigorously and stains dark gray. $\mathrm{HCl}$ and $\mathrm{KCN}$ negative. $\mathrm{FeCl}_{3}$ stains differentially and brings out etch cleavage. $\mathrm{KOH}$ negative. $\mathrm{HgCl}_{2}$ stains differentially iridescent. Specimen is from Schneeberg, Germany. Analysis by J. G. Fairchild, of the United States Geological Survey, shows Ni 15.2, Co 4.5, Fe 3.5, Cu 0.7, As 75.4, S 0.0 , insoluble 0.0, total 99.3. This corresponds approximately to a formula $(\mathrm{Ni}, \mathrm{Co})_{2} \mathrm{As}_{5}$, which can be expressed for purposes of comparison as $(\mathrm{Ni}, \mathrm{Co}) \mathrm{As}_{2.5}$. See description of smaltite-chloanthite. The different varieties of the isomorphous series chloanthite-smaltite all give the same etch tests and can be distinguished only by microchemical methods. The end members are very rare.

Corynite $(\mathrm{Ni}(\mathrm{As}, \mathrm{Sb}) \mathrm{S})$.- - Isometric; color grayish white; hardness $\mathrm{F} . \mathrm{HNO}_{3}$ effervesces and brings out etch cleavage. $\mathrm{HCl}$ and $\mathrm{KCN}$ negative. $\mathrm{FeCl}_{3}$ stains brown and brings out etch cleavage. $\mathrm{KOH}$ negative. $\mathrm{HgCl}_{2}$ stains brown to iridescent. Specimen from Olsa, Carinthia, Austria.

Maucherite $\left(\mathrm{Ni}_{3} \mathrm{As}_{2}\right)$.- Isometric; color pinkish gray, about same as cobaltite; hardness D. Some specimens isotropic, others weakly anisotropic. $\mathrm{HNO}_{3}$ instantly effervesces and stains black. $\mathrm{HCl}$ and $\mathrm{KCN}$ negative. $\mathrm{FeCl}_{3}$ quickly tarnishes iridescent. $\mathrm{KOH}$ negative. $\mathrm{HgCl}_{2}$ slowly stains brown.

Smaltite-chloanthite ((Co, Ni) $\left.\mathrm{As}_{2}\right)$.-Isometric; color gray; hardness F. $\mathrm{HNO}_{3}$ effervesces vigorously; surface stains differentially, bringing out concentric shell structure. $\mathrm{HCl}$ and $\mathrm{KCN}$ negative. $\mathrm{FeCl}_{3}$ stains differentially brown. $\mathrm{KOH}$ negative. $\mathrm{HgCl}_{2}$ stains brown to iridescent. Specimen is from Schneeberg, Germany. Analysis by J. J. Fahey, of the United States Geological Survey, shows insoluble 0.32, Ni 15.07, Co 15.83, Fe 3.69, Bi 0.86, As 63.42, total 99.19. This corresponds approximately to the formula $(\mathrm{Co}, \mathrm{Ni}) \mathrm{As}_{1.5}$. Analyses of smaltite-chloanthite show considerable variation, ranging from $(\mathrm{Co}, \mathrm{Ni}) \mathrm{As}_{1.5}$ to $(\mathrm{Co}, \mathrm{Ni}) \mathrm{As}_{3}$. The latter end member is termed skutterudite. Nickel-free varieties are rare. The average corresponds to about $(\mathrm{Co}, \mathrm{Ni}) \mathrm{As}_{2}$.

Arsenoferrite $\left(\mathrm{FeA}_{2}\right)$.- Isometric; color light gray, almost KOH neg. white; hardness F. $\mathrm{HNO}_{3}$ effervesces slowly; stains brown. $\mathrm{HgCl}_{2}$ neg. $\mathrm{HCl}$ and $\mathrm{KCN}$ negative. $\mathrm{FeCl}_{3}$ stains brown. $\mathrm{KOH}$ and $\mathrm{HgCl}_{2}$ negative. Specimen from Jachymov (Joachimsthal), Czechoslovakia. Analysis by W. F. Foshag, of the United States National Museum, shows $\mathrm{Fe} 24.88, \mathrm{Cu} 1.34, \mathrm{~Pb} 0.05, \mathrm{CaCO}_{3} 4.00, \mathrm{MgCO}_{3}$ 1.57, As 66.84, $\mathrm{S}$ 1.08, total 99.76. Very rare. Mineral is the ferriferous end member of the smaltite series $(\mathrm{Co}, \mathrm{Ni}, \mathrm{Fe}) \mathrm{As}_{2}$.

Gersdorffite (NiAsS).-Isometric; color grayish white; hardness $\mathrm{F}-. \mathrm{HNO}_{3}$ effervesces vigorously and turns black. $\mathrm{HCl}$ and $\mathrm{KCN}$ negative. $\mathrm{FeCl}_{3}$ stains dark gray to brown. $\mathrm{KOH}$ and $\mathrm{HgCl}_{2}$ negative.

Skutterudite ((Co,Ni) $\left.\mathrm{As}_{3}\right)$.-Isometric; color creamy; hardness $\mathrm{F}$. $\mathrm{HNO}_{3}$ effervesces and turns iridescent to black. $\mathrm{HCl}$ and $\mathrm{KCN}$ negative. $\mathrm{FeCl}_{3}$ stains brown. $\mathrm{KOH}$ and $\mathrm{HgCl}_{2}$ negative. Occurs as pyritohedrons with concentric shells. Specimen from type locality gave good microchemical tests for nickel and cobalt. 


\section{ANISOTROPIC}

KOH neg. $\mathrm{HgCl}_{2}$

Maucherite $\left(\mathrm{Ni}_{3} \mathrm{As}_{2}\right)$.-Color pinkish gray, about the same as cobaltite; hardness D. Some specimens isotropic; others very weakly anisotropic. $\mathrm{HNO}_{3}$ instantly effervesces and stains black. $\mathrm{HCl}$ and $\mathrm{KCN}$ negative. $\mathrm{FeCl}_{3}$ quickly tarnishes iri. descent. $\mathrm{KOH}$ negative. $\mathrm{HgCl}_{2}$ slowly stains brown. Some specimens show\& radial structure after etching.

Niccolite (NiAs).-Hexagonal; color coppery pink; hardness E. Strongly anisotropic. $\mathrm{HNO}_{3}$ tarnishes differentially with effervescence. $\mathrm{HCl}$ and $\mathrm{KCN}$ negative. $\mathrm{FeCl}_{3}$ slowly stains differentially brown to iridescent. $\mathrm{KOH}$ negative, $\mathrm{HgCl}_{2}$ stains brown to iridescent. Appears buff when in contact with breithauptite.

Plessite ((Ni,Fe) AsS).-Isometric; color white; hardness F. Weak anomaloug anisotropism. $\mathrm{HNO}_{3}$ effervesces and stains black. $\mathrm{HCl}$ and $\mathrm{KCN}$ negative. $\mathrm{FeCl}_{3}$ effervesces and stains differentially brown to iridescent. $\mathrm{KOH}$ negative. $\mathrm{HgCl}_{2}$ slowly stains light brown; some areas unaffected. Iron-bearing gersdorffite. Distinguished from gersdorffite by anisotropism and $\mathrm{HgCl}_{2}$ test.

Rammelsbergite $\left(\mathrm{NiAs}_{2}\right)$.-Orthorhombic; color white; hardness E. $\mathrm{HNO}_{3}$ effervesces, stains dark, and brings out lamellar structure. $\mathrm{HCl}$ and $\mathrm{KCN}$ nega. tive. $\mathrm{FeCl}_{3}$ stains brown. $\mathrm{KOH}$ negative. $\mathrm{HgCl}_{2}$ slowly stains brown.

\section{$\mathrm{HNO}_{3}$ eff., $\mathrm{HCl}$ neg., $\mathrm{KCN}$ neg., $\mathrm{FeCl}_{3}$ neg.}

\section{ISOTROPIC}

KOH neg. hardness $\mathrm{F}-\mathrm{H} \mathrm{HNO}_{3}$ effervesces and stains iridescent; in some $\mathbf{H g C l}_{2}$ neg. specimens brings out parallel etch structure; usually stains gray and $\mathrm{HgCl}_{2}$ negative.

to brown; some specimens negative. $\mathrm{HCl}, \mathrm{KCN}, \mathrm{FeCl}_{3}, \mathrm{KOH}$,

Pyrite $\left(\mathrm{FeS}_{2}\right)$.-Isometric; color pale brass-yellow; hardness F. $\mathrm{HNO}_{3}$ fumes tarnish; some specimens slowly effervesce; with small grains or poorly polished surfaces reaction may be entirely negative. $\mathrm{HCl}, \mathrm{KCN}, \mathrm{FeCl}_{3}, \mathrm{KOH}$, and $\mathrm{HgCl}_{2}$ negative. Aqua regia fumes tarnish; some specimens slowly effervesce. Not seratched by needle, which is best means of distinguishing from chalcopyrite.

\section{ANISOTROPIC}

KOH neg. hardness $\mathrm{F}+$. Strong anisotropism. $\mathrm{HNO}_{3}$ slowly effervesces $\mathbf{H g C l}_{2}$ neg. and stains differentially iridescent; effervescence not always observed. $\mathrm{HCl}, \mathrm{KCN}, \mathrm{FeCl}_{3}, \mathrm{KOH}$, and $\mathrm{HgCl}_{2}$ negative. Diamond-shaped cross sections frequently observed. Distinguished from löllingite by whiter color of arsenopyrite.

Löllingite (nickeliferous) ((Fe, $\left.\mathrm{Ni}) \mathrm{As}_{2}\right)$.-Orthorhombic; color galena-white; hardness E+. Strongly anisotropic. $\mathrm{HNO}_{3}$ slowly effervesces; deposits colorless octahedra; mineral tarnishes iridescent. $\mathrm{HCl}, \mathrm{KCN}, \mathrm{FeCl}_{3}, \mathrm{KOH}$, and ${ }^{-} \mathrm{HgCl}_{2}$ negative. Etch tests practically the same as lollingite. Microehemical tests show abundant nickel as well as iron.

Marcasite $\left(\mathrm{FeS}_{2}\right)$.-Orthorhombic; color pale brass-yellow; hardness Et. Strong anisotropism. $\mathrm{HNO}_{3}$ stains brown to iridescent with slow effervescence; sometimes effervescence not observed. $\mathrm{HCl}, \mathrm{KCN}, \mathrm{FeCl}_{3}, \mathrm{KOH}$, and $\mathrm{HgCl}_{9}$ negative. Prismatic. Marcasite is slightly paler in color than pyrite. The 
only certain way of distinguishing between pyrite and marcasite is by means of polarized light.

Pyrite $\left(\mathrm{FeS}_{2}\right)$.-Isometric; color pale brass-yellow; hardness F. More than 99 per cent of pyrite specimens are isotropic; very rarely, however, polished sections show pyritohedrons with the color of pyrite rather than of marcasite, each pyritohedron being a mosaic of grains of weak anisotropism and diverse orientation. $\mathrm{HNO}_{3}$ fumes tarnish; some specimens stain slightly; drop effervesces slowly. Other reagents negative. Specimen examined is from Cerro de Pasco, Peru. The color and the square rather than prismatic cross sections are sufficient criteria to identify mineral as pyrite.

\section{$\mathrm{HNO}_{3}, \mathrm{HCl}, \mathrm{KCN}$ neg., $\mathrm{FeCl}_{3}$}

ISOTROPIC

Psilomelane $\left(\mathrm{Mn}_{2} \mathrm{MnO}_{5}\right)$.-Amorphous; color gray; hardкоH neg. ness D+. $\mathrm{HNO}_{3}$ stains light brown; fumes tarnish. $\mathrm{HCl}$ $\mathrm{HgCl}_{2}$ neg. stains brown to black; action more intense than that of $\mathrm{HNO}_{3}$. $\mathrm{KCN}$ negative. $\mathrm{FeCl}_{3}$ stains light brown. $\mathrm{KOH}$ and $\mathrm{HgCl}_{2}$ negative. $\mathrm{H}_{2} \mathrm{O}_{2}$ effervesces vigorously without etching. Massive, botryoidal, or reniform structures. Polished surfaces commonly show concentric banding.

\section{$\mathrm{HNO}_{3}, \mathrm{HCl}, \mathrm{KCN}$ neg., $\mathrm{FeCl}_{3}$ neg.}

\section{ANISOTROPIC}

Hausmannite $\left(\mathrm{Mn}_{3} \mathrm{O}_{4}\right)$.-Hexagonal; color white with a sugKOH neg. gestion of blue; hardness D-. Strongly anisotropic; most $\mathrm{HgCl}_{2}$ neg. specimens show multiple twinning resembling that of plagioclase. $\mathrm{HNO}_{3}$ fumes tarnish; some specimens negative. $\mathrm{HCl}$ stains differentially brown; some areas negative. $\mathrm{KCN}, \mathrm{FeCl}_{3}, \mathrm{KOH}$, and $\mathrm{HgCl}_{2}$ negative. $\mathrm{H}_{2} \mathrm{O}_{2}$ effervesces slowly without staining surface. Good basal cleavage. Powder and internal reflection brick-red to deep red. Takes a high polish. Can be scratched with a sharp needle.

\section{$\mathrm{HNO}_{3}$, $\mathrm{HCl}$ neg., $\mathrm{KCN}$ neg., $\mathrm{FeCl}_{3}$}

\section{ISOTROPIC}

KOH neg. gray; hardness $\mathrm{D}+. \mathrm{HNO}_{3}$ slowly tarnishes iridescent; some$\mathrm{HgCl}_{2}$ times effervesces slightly. $\mathrm{HCl}$ and $\mathrm{KCN}$ negative. $\mathrm{FeCl}_{3}$ stains slightly and brings out scratches. $\mathrm{KOH}$ negative. $\mathrm{HgCl}_{2}$ leaves a brown deposit which rubs off easily. Specimen from Horace Porter mine, Gunnison County, Colo. Analysis by J. G. Fairchild, of the United States Geological Survey, shows Co 10.98, Ni 5.14, Fe 5.82, As 75.30, insoluble 1.44, total 98.68 .

Kallitite (Ni(Sb,Bi) S).-Isometric; color gray; hardness $\mathrm{D}$. KOH neg. $\mathrm{HNO}_{3}$ tarnishes iridescent. $\mathrm{HCl}$ and $\mathrm{KCN}$ negative. $\mathrm{FeCl}_{3}$ $\mathrm{HgCl}_{2}$ neg. stains faintly, almost negative. $\mathrm{KOH}$ and $\mathrm{HgCl}_{2}$ negative. From Schoenstein, Germany. Very rare. According to Thom$80 n,{ }^{18}$ kallilite is a mixture. The present writer's specimen is homogeneous and contains all three metallic elements.

\footnotetext{
18 Thomson, Ellis, A mineralographic study of the pyrite group: Toronto Univ. Studies, Geol. ser., No. 12, p. 36, 1921.
} 
Ullmanite (NiSbS).-Isometric; color galena-white; hardness E. $\mathrm{HNO}_{3}$ staing brown to irridescent. $\mathrm{HCl}$ and $\mathrm{KCN}$ negative. $\mathrm{FeCl}_{3}$ stains light brown. $\mathrm{KOH}$ and $\mathrm{HgCl}_{2}$ negative. Distinctly softer than gersdorffite. Has well-defines cleavage and shows triangular pits.

Willyamite ((Co, Ni) SbS). - Isometric; color gray; hardness $\mathrm{E} . \mathrm{HNO}_{3}$ tarnishes iridescent; seems to effervesce slightly. $\mathrm{HCl}$ and $\mathrm{KCN}$ negative. $\mathrm{FeCl}_{3}$ stains faintly, almost negative. $\mathrm{KOH}$ and $\mathrm{HgCl}_{2}$ negative. From Broken Hill, Australia. Rare.

$\mathrm{HNO}_{3}$, $\mathrm{HCl}$ neg., KCN neg., $\mathrm{FeCl}_{3}$

ANISOTROPIC

KOH neg. $\mathrm{HgCl}_{2}$ neg.

Breithauptite (NiSb).-Hexagonal; color coppery pink; hard. ness E. Strong anisotropism. $\mathrm{HNO}_{3}$ stains differentially iridescent. $\mathrm{HCl}$ and $\mathrm{KCN}$ negative. $\mathrm{FeCl}_{3}$ stains differentially iridescent. $\mathrm{KOH}$ and $\mathrm{HgCl}_{2}$ negative.

$\mathrm{HNO}_{3}$, $\mathrm{HCl}$ neg., $\mathrm{KCN}$ neg., $\mathrm{FeCl}_{3}$ neg.

\section{ANISOTROPIC}

Arsenopyrite (FeAsS).-Orthorhombic; color galena-white; KОH neg. hardness $\mathrm{F}+$. Strong anisotropism. $\mathrm{HNO}_{3}$ stains differentially $\mathrm{HgCl}_{2}$ neg. iridescent. $\mathrm{HCl}, \mathrm{KCN}, \mathrm{FeCl}_{3}, \mathrm{KOH}, \mathrm{HgCl}_{2}$ negative. Cross by whiter color. sections frequently observed. Distinguished from löllingite

Cobaltite (CoAsS).--Isometric; color pinkish gray; hardness G. Some specimens isotropic; others faintly anisotropic. $\mathrm{HNO}_{3}$ fumes tarnish; after long leaching stains differentially. $\mathrm{HCl}, \mathrm{KCN}, \mathrm{FeCl}_{3}, \mathrm{KOH}$, and $\mathrm{HgCl}_{2}$ negative. Usually crystallizes in cubes and pyritohedrons.

Glaucodot ((Co,Fe)AsS).-Orthorhombic; color white; hardness F. Weak anisotropism. $\mathrm{HNO}_{3}$ stains iridescent. $\mathrm{HCl}, \mathrm{KCN}, \mathrm{FeCl}_{3}, \mathrm{KOH}$, and $\mathrm{HgCl}_{3}$ negative.

Leucopyrite $\left(\mathrm{Fe}_{3} \mathrm{As}_{4}\right)$.-Orthorhombic; color white; hardness F. Strong anisotropism. $\mathrm{HNO}_{3}$ stains brown; solution deposits tetrahedral and octahedral crystals. $\mathrm{HCl}, \mathrm{KCN}, \mathrm{FeCl}_{3}, \mathrm{KOH}$, and $\mathrm{HgCl}_{2}$ negative. Distinguished with difficulty from arsenopyrite and löllingite.

Löllingite $\left(\mathrm{FeAs}_{2}\right)$. - Orthorhombic; color grayish white; hardness $\mathrm{F}-$. Strong anisotropism. $\mathrm{HNO}_{3}$ stains brown to iridescent; fumes tarnish. $\mathrm{HCl}, \mathrm{KCN}$, $\mathrm{FeCl}_{3}, \mathrm{KOH}$, and $\mathrm{HgCl}_{2}$ negative.

Marcasite $\left(\mathrm{FeS}_{2}\right)$.- Orthorhombic; color pale brass-yellow; hardness E†. Strong anisotropism. $\mathrm{HNO}_{3}$ stains brown to iridescent; with larger grains sometimes gives slow effervescence; with smaller grains effervescence usually not observed. $\mathrm{HCl}, \mathrm{KCN}, \mathrm{FeCl}_{3}, \mathrm{KOH}$, and $\mathrm{HgCl}_{2}$ negative. Prismatic. Color paler than pyrite. The only certain way of distinguishing between marcasite and pyrite is by means of polarized light.

Pyrite $\left(\mathrm{FeS}_{2}\right)$.-Isometric; color pale brass-yellow; hardness $\mathrm{F}$. More than 99 per cent of pyrite specimens are isotropic; very rarely, however, polished sections show pyritohedrons with the color of pyrite rather than of marcasite, esech pyritohedron being a mosaic of weakly anisotropic grains. $\mathrm{HNO}_{3}$ fumes tarnish. Other reagents negative. Specimen examined is from Cerro de Pasco, Peru. The color and the square rather than prismatic cross sections of crystals are sufficient criteria to identify the mineral as pyrite. 
$\mathrm{HNO}_{3}$, HCl neg., KCN neg., $\mathrm{FeCl}_{3}$ neg.

ISOTROPIC

Bravoite $\left((\mathrm{Fe}, \mathrm{Ni}) \mathrm{S}_{2}\right)$.--Isometric; color pale violet; hardness KOH neg. E. $\mathrm{HNO}_{3}$ fumes tarnish; mineral stains iridescent and brings $\mathrm{HgCl}_{2}$ neg. out zonal structure. $\mathrm{HCl}, \mathrm{KCN}, \mathrm{FeCl}_{3}, \mathrm{KOH}$, and $\mathrm{HgCl}_{2}$ negative. Specimen from Mechernich, Germany. Occurs in zoned pyritohedrons, with alternate yellow and violet shells. The yellow shells are pyrite with only a trace of nickel, and the violet shells consist of bravoite. The analyses probably show too much iron in the bravoite owing to the mechanical admixture of pyrite. ${ }^{40}$

Cobaltite (CoAsS).-Isometric; color pinkish gray; hardness G. Some specimens isotropic; others weakly anisotropic. $\mathrm{HNO}_{3}$, some specimens stain differentially iridescent after long etching and fumes tarnish; other specimens negative. $\mathrm{HCl}, \mathrm{KCN}, \mathrm{FeCl}_{3}, \mathrm{KOH}$, and $\mathrm{HgCl}_{2}$ negative. Usually crystallizes in cubes and pyritohedrons.

Corynite $(\mathrm{Ni}(\mathrm{As}, \mathrm{Sb}) \mathrm{S})$.- - Isometric; color gray; hardness $\mathrm{E}+. \mathrm{HNO}_{3}$ stains iridescent; fumes tarnish. $\mathrm{HCl}, \mathrm{KCN}, \mathrm{FeCl}_{3}, \mathrm{KOH}$, and $\mathrm{HgCl}_{2}$ negative. Specimen is from Chatham, Conn. Analysis by J. G. Fairchild: Ni 31.6, Co $0.7, \mathrm{Fe} 3.9, \mathrm{As} 34.9, \mathrm{Sb} 9.1, \mathrm{Bi} 0.4, \mathrm{~S} 17.1$, total 97.2

Pyrite $\left(\mathrm{FeS}_{2}\right)$.-Isometric; color pale brass-yellow; hardness $\mathrm{F}$. $\mathrm{HNO}_{3}$ fumes tarnish; some specimens slowly effervesce; with small grains or poorly polished surfaces reaction may be entirely negative. $\mathrm{HCl}, \mathrm{KCN}, \mathrm{FeCl}_{3}, \mathrm{KOH}$, and $\mathrm{HgCl}_{2}$ negative. Aqua regia fumes tarnish; some specimens slowly effervesce. Not scratched by needle, which is best means of distinguishing from chalcopyrite.

Willyamite ((Co,Ni) SbS).-Isometric; color gray; hardness $\mathrm{E} . \quad \mathrm{HNO}_{3}$ tarnishes iridescent; seems to effervesce slightly. $\mathrm{HCl}$ and $\mathrm{KCN}$ negative. $\mathrm{FeCl}_{3}$ seems to stain faintly; this reaction is easily overlooked. $\mathrm{KOH}$ and $\mathrm{HgCl}_{2}$ negative.

\section{$\mathrm{HNO}_{3}$ neg., $\mathrm{HCl}, \mathrm{KCN}$ neg., $\mathrm{FeCl}_{3}$ neg.}

ISOTROPIC

Franklinite ((Fe, $\left.\mathrm{Zn}, \mathrm{Mn}) \mathrm{O} .(\mathrm{Fe}, \mathrm{Mn})_{2} \mathrm{O}_{3}\right)$. - - Isometric; color gray, KOH neg. about the same as magnetite; hardness G. $\mathrm{HNO}_{3}$ negative. $\mathrm{HCl}$ $\mathrm{HgCl}_{2}$ neg. usually negative, but fumes sometimes tarnish; solution turns yellow. $\mathrm{KCN}, \mathrm{FeCl}_{3}, \mathrm{KOH}$, and $\mathrm{HgCl}_{2}$ negative. Aqua regia solution turns yellow. Dark-brown powder when scratched. Powder is somewhat magnetic. '(See description of chromite, p. 102.)

Magnetite $\left(\mathrm{Fe}_{3} \mathrm{O}_{4}\right)$.- Isometric; color gray; hardness $\mathrm{F} . \mathrm{HNO}_{3}$ negative. $\mathrm{HCl}$, on smooth surfaces larger than drop, fumes tarnish; solution turns yellow. $\mathrm{KCN}, \mathrm{FeCl}_{3}, \mathrm{KOH}$, and $\mathrm{HgCl}_{2}$ negative. Aqua regia solution turns yellow. Black powder when scratched. Powder is magnetic. (See description of chromite, p. 102.)

\section{ANISOTROPIC}

Hausmannite $\left(\mathrm{Mn}_{3} \mathrm{O}_{4}\right)$.- - Hexagonal; color white with a sugKOH neg. gestion of blue; hardness $\mathrm{D}-$. Strongly anisotropic; most $\mathrm{HgCl}_{2}$ neg. specimens show multiple twinning resembling that of plagioclase. $\mathrm{HNO}_{3}$ fumes tarnish; some specimens negative. $\mathrm{HCl}$ stains differentially light brown; some areas negative. $\mathrm{KCN}, \mathrm{FeCl}_{3}, \mathrm{KOH}$, and $\mathrm{HgCl}_{2}$

\footnotetext{
"See Short, M. N., and Shannon, E. V., Violarite and other rare nickel sulphides: Am. Mineralogist, vol. 15, pp. 12-14, 1930.
} $15122-31-8$ 
negative. $\mathrm{H}_{2} \mathrm{O}_{2}$ effervesees slowly without staining surface. Good basal clear. age. Powder and internal reflection brick-red to deep red. Takes a high polish, Can be scratched with a sharp needle.

\section{$\mathrm{HNO}_{3}$ neg., $\mathrm{HCl}$ neg., $\mathrm{KCN}$ neg., $\mathrm{FeCl}_{3}$}

\section{ISOTROPIC}

Uraninite (uranate of $\mathrm{U}, \mathrm{Pb}$, etc.).-Isometric; color dark gray; Kон neg. hardness $\mathrm{G}$. $\mathrm{HNO}_{3}, \mathrm{HCl}$, and $\mathrm{KCN}$ negative. $\mathrm{FeCl}_{3}$ slowly $\mathrm{HgCl}_{2}$ neg. tarnishes to still darker brownish gray. $\mathrm{KOH}, \mathrm{HgCl}_{2}$, and aqua regia negative. Massive. Powder greenish brown to black.

\section{$\mathrm{HNO}_{3}$ neg., $\mathrm{HCl}$ neg., $\mathrm{KCN}$ neg., $\mathrm{FeCl}_{3}$ neg., aqua regia}

\section{ISOTROPIC}

Pyrile $\left(\mathrm{FeS}_{2}\right)$.--Isometric; color pale brass-yellow; hardness $\mathrm{P}$. KOH neg. $\quad \mathrm{HNO}_{3}$ fumes tarnish; some specimens slowly effervesce; with $\mathrm{HgCl}_{2}$ neg. small grains or poorly polished surfaces reaction may be entirely negative. $\mathrm{HCl}, \mathrm{KCN}, \mathrm{FeCl}_{3}, \mathrm{KOH}$, and $\mathrm{HgCl}_{2}$ negative. Aqua regia fumes tarnish; some specimens slowly effervesce. Not scratched by needle, which is best means of distinguishing from chalcopyrite.

Sperrylite $\left(\mathrm{PtAs}_{2}\right)$.-Isometric; color white; hardness F. $\mathrm{HNO}_{3}, \mathrm{HCl}, \mathrm{KCN}$, $\mathrm{FeCl}_{2}, \mathrm{KOH}$, and $\mathrm{HgCl}_{2}$ negative. Aqua regia effervesces slightly and stains light brown in places. Occurs as small isometric crystals. Only locality Sudbury, Ontario.

\section{$\mathrm{HNO}_{3}$ neg., $\mathrm{HCl}$ neg. $\mathrm{KCN}$ neg., $\mathrm{FeCl}_{3}$ neg., aqua regia neg.}

\section{ISOTROPIC}

Braunite $\left(3 \mathrm{Mn}_{2} \mathrm{O}_{3} \cdot \mathrm{MnSiO}_{2}\right)$.-Tetragonal; color gray; hardness

$\mathbf{K O H}$ meg. G. Apparently isotropic. $\mathrm{HNO}_{3}, \mathrm{HCl}, \mathrm{KCN}, \mathrm{FeCl}_{3}, \mathrm{KOH}$ $\mathrm{HgCl}_{2}$ neg. $\mathrm{HgCl}_{2}$, and aqua regia negative. $\mathrm{H}_{2} \mathrm{O}_{2}$ slowly effervesces without staining surface. Gives dark-brown powder when scratched. Frequently associated with hausmannite.

Chromite ( $\mathrm{FeO} . \mathrm{Cr}_{2} \mathrm{O}_{3}$ ).-Isometric; color gray; hardness $\mathrm{G}+$. Negative to sull reagents, including $\mathrm{H}_{2} \mathrm{O}_{2}$. Chromite has a fairly light-brown powder; franklinite has a dark-brown and magnetite a black powder. Magnetite is much more strongly magnetic than chromite. Magnetite particles will stand on end whem a magnetic needle approaches them. Chromite and franklinite will cling to a magnetized needle when the needle comes into contact with them.

Cobaltite (CoAsS).-Isometric; color pinkish gray; hardness G. Some specimeas isotropic; others show weak anisotropism. $\mathrm{HNO}_{3}$, some specimens, fumes tarnish and mineral stains differentially iridescent after long leaching; other specimens negative. $\mathrm{HCl}, \mathrm{KCN}, \mathrm{FeCl}_{3}, \mathrm{KOH}, \mathrm{HgCl}_{2}$, and aqua regia negative. Usually erystallizes in cubes and pyritohedrons.

Franklinite $\left((\mathrm{Fe}, \mathrm{Zn}, \mathrm{Mn}) \mathrm{O} .(\mathrm{FeMn})_{2} \mathrm{O}_{3}\right)$.--Isometric; color gray, about the same as magnetite; hardness $\mathrm{G} . \mathrm{HNO}_{3}$ negative. $\mathrm{HCl}$ usually negative, but fumes sometimes tarnish; solution turns yellow. $\mathrm{KCN}, \mathrm{FeCl}_{3}, \mathrm{KOH}$, and $\mathrm{HgCl}_{3}$ negative. Aqua regia solution turns yellow. Dark-brown powder when seratehed. Powder is somewhat magnetic. (See description of chromite.)

Hematite $\left(\mathrm{Fe}_{2} \mathrm{O}_{3}\right)$.- Hexagonal; color gray; hardness G. Negative to all reagents. Fine-grained and earthy varieties of hematite will give a red powder 
when scratched with a needle. Some specularite specimens, however, yield a metallic powder.

Magnetite $\left(\mathrm{Fe}_{3} \mathrm{O}_{4}\right)$.-Isometric; color gray; hardness F. $\mathrm{HNO}_{3}$ negative. $\mathrm{HCl}$ usually negative; on smooth surfaces larger than drop, fumes tarnish. $\mathrm{KCN}, \mathrm{FeCl}_{3}, \mathrm{KOH}$, and $\mathrm{HgCl}_{2}$ negative. Aqua regia solution turns yellow. Black powder when scratched. Powder is magnetic. (See description of chromite, p. 102.)

\section{ANISOTROPIC}

Cassiterite $\left(\mathrm{SnO}_{2}\right)$.-Tetragonal; color gray; hardness G. Negative to all reagents. Can not be scratched by needle. Resin-colored internal reflection; not always seen. Color of hand specimen white to yellow.

Cobaltite (CoAsS).-Isometric; color pinkish gray; hardness G-. Usually shows weak anomalous anisotropism. Some specimens negative to all reagents. Ferberite $\left(\mathrm{FeWO}_{4}\right)$.-Monoclinic; color gray, like sphalerite; hardness E. Strong anisotropism. Negative to all reagents. Powder dark, almost metallic. Goethite $\left(\mathrm{Fe}_{2} \mathrm{O}_{3} \cdot \mathrm{H}_{2} \mathrm{O}\right)$.- Orthorhombic; color gray; hardness E. Moderately strong anisotropism. $\mathrm{HNO}_{3}$ negative. $\mathrm{HCl}$ doubtful; fumes tarnish slightly after long standing. $\mathrm{KCN}, \mathrm{FeCl}_{3}, \mathrm{KOH}$, and $\mathrm{HgCl}_{2}$ negative. Aqua regia same as $\mathrm{HCl}$. Gives yellow to orange-colored powder.

Hematite (specular) $\left(\mathrm{Fe}_{2} \mathrm{O}_{3}\right)$.- - Hexagonal; color galena-white; hardness G. Negative to all reagents. Habit platy.

Hübnerite $\left(\mathrm{MnWO}_{4}\right)$.-Monoclinic; color gray, like sphalerite; hardness E. Strong anisotropism. Can readily be scratched by needle. Negative to all reagents. Gives yellow powder and dark-red internal reflection.

Ilmenite $\left(\mathrm{FeTiO}_{3}\right)$. - Hexagonal; color brownish gray; some specimens have suggestion of violet color; hardness G. Negative to all reagents. Commonly intergrown with specularite and magnetite.

Polianite $\left(\mathrm{MnO}_{2}\right)$.-Tetragonal; color gray; hardness G. Strongly anisotropic; gives four extinctions. $\mathrm{HNO}_{3}, \mathrm{HCl}, \mathrm{KCN}, \mathrm{FeCl}_{3}, \mathrm{KOH}, \mathrm{HgCl}_{2}$, and aqua regia negative. $\mathrm{H}_{2} \mathrm{O}_{2}$ effervesces vigorously without staining surface. Prismatic. Very rare; found only at Platten, Bohemia. Resembles pyrolusite closely but distinguished by hardness.

Rutile $\left(\mathrm{TiO}_{2}\right)$.-Tetragonal; color gray, similar to sphalerite; hardness $\mathrm{G}-$. Moderately strong anisotropism. Negative to all reagents. Orange to red internal reflection and powder. Almost invariably prismatic.

Wolframite $\left((\mathrm{Fe}, \mathrm{Mn}) \mathrm{WO}_{2}\right)$.-Monoclinic; color gray, like sphalerite; hardness E. Negative to all reagents. Shows cleavage in two directions. Gives darkbrown powder. Distinguished from hübnerite and ferberite only by chemical tests.

\section{BINNENTHAL MINERALS}

The following data on minerals that have been reported only from Binnenthal, Switzerland, are taken from Davy and Farnham's text. The present author was unable to obtain specimens of these minerals. The effect of polarized light is inferred from the crystal system as given in Dana's "System of mineralogy."

Dufrenoysite (2PbS. $\mathrm{As}_{2} \mathrm{~S}_{3}$ ).-Orthorhombic; color gray; hardness C. Anisotropic. $\mathrm{HNO}_{3}, \mathrm{HCl}, \mathrm{KCN}$, and $\mathrm{FeCl}_{3}$ negative. KOH tarnishes iridescent and darkens. Gives reddish-brown powder. 
Baumhauerite (4PbS.3 $\mathrm{As}_{2} \mathrm{~S}_{3}$ ).-Monoclinic; color grayish white; hardness $\mathrm{C}$, Anisotropic. $\mathrm{HNO}_{3}$ negative; sometimes tarnishes along cracks; rubs clean. $\mathrm{HCl}$ negative. $\mathrm{KCN}$ negative; sometimes tarnishes along cracks. $\quad \mathrm{FeCl}_{3}$ negative, $\mathrm{KOH}$ tarnishes iridescent. $\mathrm{HgCl}_{2}$ slowly tarnishes brown. Brittle. Internal reflection red. Gives brown powder.

Lengenbachite (6PbS. $\left(\mathrm{Ag}, \mathrm{Cu}{ }_{2} \mathrm{~S} .2 \mathrm{As}_{2} \mathrm{~S}_{3}\right)$.-Triclinic; color gray; hardness $\mathrm{B}$, Anisotropic. $\mathrm{HNO}_{3}$ practically negative, but sometimes tarnishes slightly. $\mathrm{HCl}, \mathrm{KCN}, \mathrm{FeCl}_{3}, \mathrm{KOH}$, and $\mathrm{HgCl}_{2}$ negative.

Seligmannite $\left(\mathrm{Cu}_{2} \mathrm{~S} .2 \mathrm{PbS} . \mathrm{As}_{2} \mathrm{~S}_{3}\right)$.- Orthorhombic; color gray; hardness $\mathrm{C}$. Anisotropic. $\mathrm{HNO}_{3}, \mathrm{HCl}, \mathrm{KCN}, \mathrm{FeCl}_{3}$, and $\mathrm{HgCl}_{2}$ negative. $\mathrm{KOH}$ slowly tarnishes iridescent.

\section{DISCREDITED OR DOUBTFUL MINERALS 50}

Chilenite $\left(\mathrm{Ag}_{6} \mathrm{Bi}\right)$.- Specimen from Copiapo, Chile, the type locality, is an intergrowth of native silver and cuprite $\left(\mathrm{Cu}_{2} \mathrm{O}\right)$.

Chiviatite $\left(2 \mathrm{PbS} 3 \mathrm{Bi}_{2} \mathrm{~S}_{3}\right)$. - Specimens from Chiviato, Peru, the type locality, proved to be a mixture of bismuthinite and various copper minerals.

Comuccite.-Formula not given by describer, but analysis is as follows: $\mathrm{Pb}$ 37.86, Fe 3.99, Sb 36.01, S 21.54, total 99.40. Specimen from San Giorgio, Sardinia, the type locality, is identical in physical properties and etch tests with jamesonite (4PbS.FeS.3Sb $\mathrm{S}_{3}$ ).

Cuprobismutite $\left(3 \mathrm{Cu}_{2} \mathrm{~S}_{4} 4 \mathrm{Bi}_{2} \mathrm{~S}_{3}\right)$. - Specimen from Halls Valley, Colo., the type locality, is identical with emplectite $\left(\mathrm{Cu}_{2} \mathrm{~S} \cdot \mathrm{Bi}_{2} \mathrm{~S}_{3}\right)$.

Dognacskaite $(\mathrm{Cu}, \mathrm{Bi}$, and $\mathrm{S})$.- -Specimens from Dognacska, Hungary, the type locality, proved to be a mixture of bismuthinite and chalcocite and other copper sulphides.

Epiboulangerite $\left(3 \mathrm{PbS} . \mathrm{Sb}_{2} \mathrm{~S}_{5}\right)$. - Identical in physical properties and etch tests with boulangerite. The formula $\mathrm{Sb}_{2} \mathrm{~S}_{5}$ seems illogical, and the method of analygis employed was questionable.

Guejarite $\left(\mathrm{Cu}_{2} \mathrm{~S} .2 \mathrm{Sb}_{2} \mathrm{~S}_{3}\right)$.-Identical with chalcostibite $\left(\mathrm{Cu}_{2} \mathrm{~S} . \mathrm{Sb}_{2} \mathrm{~S}_{3}\right)$. - $\left(\mathrm{Seet}^{2}\right.$ Dana's "System of mineralogy," Appendix I, p. 16.)

Hauchecornite $\left((\mathrm{Ni}, \mathrm{Co})_{7}(\mathrm{~S}, \mathrm{Sb}, \mathrm{Bi})_{8}\right)$. - Specimen from Hamm an der Sieg, Prussia, the type locality, is a mixture of two unknown minerals with the following properties:

1. Contains nickel, bismuth, and sulphur. Gave negative tests for cobalt: and antimony. Color pink; hardness about $\mathrm{E}$ (Talmage). Moderate anisotropism. $\mathrm{HNO}_{3}$ slowly stains brown to iridescent. $\mathrm{HCl}, \mathrm{KCN}, \mathrm{FeCl}_{3}, \mathrm{KOH}$, and $\mathrm{HgCl}_{2}$ negative.

2. Contains nickel, arsenic, antimony, and sulphur. Gave negative teste for cobalt and bismuth. Color gray with tinge of yellow; hardness about $\mathbb{B}$. Strong anisotropism. $\mathrm{HNO}_{3}$ slowly stains brown with slight effervescence. $\mathrm{HCl}, \mathrm{KCN}, \mathrm{FeCl}_{3}, \mathrm{KOH}$, and $\mathrm{HgCl}_{2}$ negative.

Hengleinite $\left((\mathrm{Fe}, \mathrm{Ni}, \mathrm{Co}) \mathrm{S}_{2}\right)$. - Specimen from Musen, Westphalia, the type locality, is a mixture of siegenite $\left((\mathrm{Ni}, \mathrm{Co})_{3} \mathrm{~S}_{4}\right)$ and pyrite.

Kalgoorlite $\left(\mathrm{HgAu}_{2} \mathrm{Ag}_{6} \mathrm{Te}_{6}\right)$.- Specimen from Kalgoorlie, Western Australia, the type locality, proved to be coloradoite ( $\mathrm{HgTe})$.

Lehrbachite (PbSe,HgSe) and zorgite.-Do not exist. ${ }^{51}$

Limonite (mixture of iron oxides).-Coarse-grained varieties are anisotropic; stalactitic limonite is usually amorphous and isotropic. Limonite is a mixture

80 The formula given after each mineral is the one which is usually given in the literature.

11 Frebold, Georg, Über einige Selenerze und ihre Paragenesen in Harz: Centralbl. Mineralogie, 19a, Abt. A, pp. 16-32. 
of oxides of iron, usually hematite and goethite; some varieties also contain jarosite. It is always of supergene origin and is most characteristically found in westhered outcrops. Gives yellow to orange-colored powder.

Onofrite $(\mathrm{Hg}(\mathrm{S}, \mathrm{Se}))$. - Specimen from Marysvale, Utah, is a mixture of tie. mannite (HgSe) and sphalerite $(\mathrm{ZnS})$.

Polyargyrite $\left(12 \mathrm{Ag}_{2} \mathrm{~S} \mathrm{Sb}_{2} \mathrm{~S}_{3}\right)$. - Specimen from Wolfach, Baden, the type locality, is s mixture of argentite $\left(\mathrm{Ag}_{2} \mathrm{~S}\right)$ and tetrahedrite $\left(5 \mathrm{Cu}_{2} \mathrm{~S} .2(\mathrm{Cu}, \mathrm{Fe}, \mathrm{Zn}) \mathrm{S} .2 \mathrm{Sb}_{2} \mathrm{~S}_{3}\right)$.

Regnolite (5(CuS, $\left.\mathrm{FeS}, \mathrm{ZnS}) . \mathrm{As}_{2} \mathrm{~S}_{3}\right)$. - Specimen from Cajamarca, Peru, the type locality, is tennantite $\left(5 \mathrm{Cu}_{2} \mathrm{~S} \cdot 2(\mathrm{Cu}, \mathrm{Fe}, \mathrm{Zn}) \mathrm{S} \cdot 2 \mathrm{As}_{2} \mathrm{~S}_{3}\right)$.

Tapalpite $\left(3 \mathrm{Ag}_{2}(\mathrm{~S}, \mathrm{Te}) \cdot \mathrm{Bi}_{2}(\mathrm{~S}, \mathrm{Te})_{3}\right)$.- Specimen from Tapalpa, Mexico, the type locality, is an intergrowth of tetradymite $\left(\mathrm{Bi}_{2}(\mathrm{Te}, \mathrm{S})_{3}\right)$ and argentite $\left(\mathrm{Ag}_{2} \mathrm{~S}\right)$. Turgite $\left(2 \mathrm{Fe}_{2} \mathrm{O}_{3} \cdot \mathrm{H}_{2} \mathrm{O}\right)$. - Is a fibrous variety of hematite $\left(\mathrm{Fe}_{2} \mathrm{O}_{3}\right) .{ }^{52}$

a see Posnjak, E., and Merwin, H. E., The system ferric oxide-sulphur trioxide-water: Am. Chem. Soc. Jour., vol. 44, p. 1971, 1922. 



\section{INDEX OF MINERAIS GROUPED BY ELEMENTS}

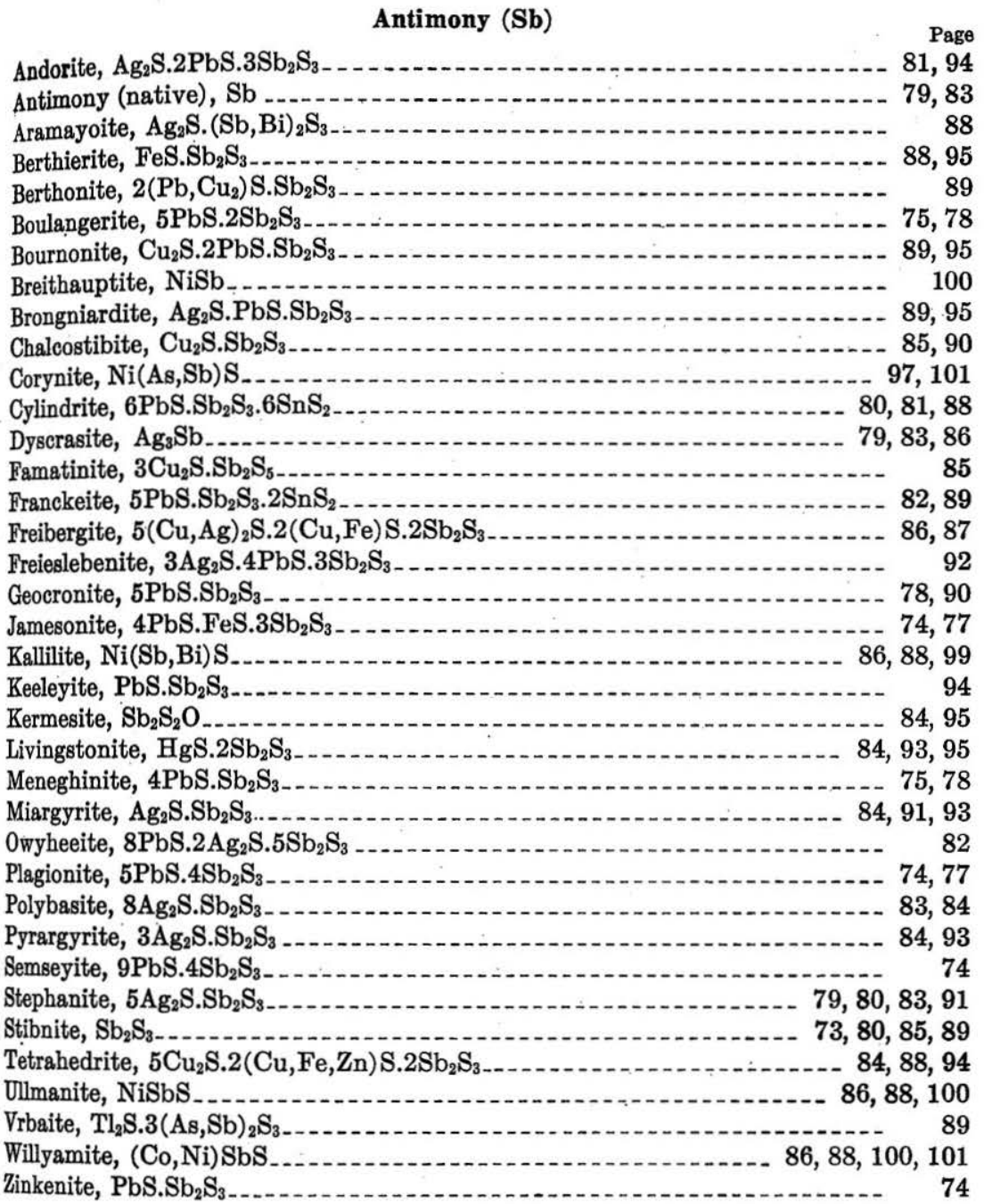

Arsenic (As)

Algodonite, $\mathrm{Cu}_{8} \mathrm{As}$

Arsenargentite, $\mathrm{Ag}_{3} \mathrm{As}$

Arsenic (native), As....

Arsenoferrite, FeAs ${ }_{2} \ldots$

Arsenopyrite, FeAsS . . . 100 
Baumhauerite, $4 \mathrm{PbS} .3 \mathrm{As}_{2} \mathrm{~S}_{3}$

Chloanthite-smaltite, $(\mathrm{Ni}, \mathrm{Co}) \mathrm{As}_{2} \ldots$

Cobaltite, CoAsS

Corynite, Ni(As,Sb)S...

Domeykite, $\mathrm{Cu}_{3} \mathrm{As}$

Dufrenoysite, $2 \mathrm{PbS} . \mathrm{As}_{2} \mathrm{~S}_{3} \ldots \ldots \ldots . . .103$

Enargite, $3 \mathrm{Cu}_{2} \mathrm{~S} . \mathrm{As}_{2} \mathrm{~S}_{5}$

Gersdorffite, NiAsS

Glaucodot, (Co,Fe)AsS . . .

Guitermanite, $3 \mathrm{PbS} . \mathrm{As}_{2} \mathrm{~S}_{3} \ldots$

Huntilite, AgAs

Jordanite, $4 \mathrm{PbS} . \mathrm{As}_{2} \mathrm{~S}_{3}$

Legenbachite, 6PbS. $\left(\mathrm{Ag}, \mathrm{Cu}{ }_{2} \mathrm{~S} .2 \mathrm{As}_{2} \mathrm{~S}_{3} \ldots \ldots \ldots\right.$

Leucopyrite, $\mathrm{Fe}_{3} \mathrm{As}_{4}$

Löllingite, $\mathrm{FeAs}_{2}$

Lorandite, $\mathrm{Tl}_{2} \mathrm{~S} . \mathrm{As}_{2} \mathrm{~S}_{3} \ldots$

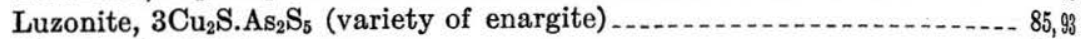

Maucherite, $\mathrm{Ni}_{3} \mathrm{As}_{2}$

Niccolite, NiAs_.

Orpiment, $\mathrm{As}_{2} \mathrm{~S}_{3}$

Pearcite, $8 \mathrm{Ag}_{2} \mathrm{~S} . \mathrm{As}_{2} \mathrm{~S}_{3}$

Plessite, $(\mathrm{Ni}, \mathrm{Fe}) \mathrm{AsS}$

Proustite, $3 \mathrm{Ag}_{2} \mathrm{~S} . \mathrm{As}_{2} \mathrm{~S}_{3}$

Rammelsbergite, $\mathrm{NiAs}_{2}$

Rathite, $3 \mathrm{PbS} .2 \mathrm{As}_{2} \mathrm{~S}_{3}$

Realgar, AsS . . .

Seligmannite, $\mathrm{Cu}_{2} \mathrm{~S} .2 \mathrm{PbS} . \mathrm{As}_{2} \mathrm{~S}_{3} \ldots \ldots \ldots$

Skutterudite, $(\mathrm{Co}, \mathrm{Ni}) \mathrm{As}_{3}$

Smaltite-chloanthite, $(\mathrm{Co}, \mathrm{Ni}) \mathrm{As}_{2} \ldots \ldots . . .19$

Sperrylite, $\mathrm{PtAs}_{2} \ldots \ldots \ldots$

Tennantite, $5 \mathrm{Cu}_{2} \mathrm{~S} .2(\mathrm{Cu}, \mathrm{Fe}, \mathrm{Zn}) \mathrm{S} .2 \mathrm{As}_{2} \mathrm{~S}_{3} \ldots \ldots \ldots . . . .68$

Vrbaite, $\mathrm{Tl}_{2} \mathrm{~S} .3(\mathrm{As}, \mathrm{Sb})_{2} \mathrm{~S}_{3} \ldots \ldots$

Whitneyite, $\mathrm{Cu}_{9} \mathrm{As}$

Bismuth (Bi)

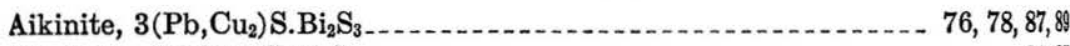

Alaskaite, $\mathrm{Ag}_{2} \mathrm{~S} . \mathrm{PbS} . \mathrm{Bi}_{2} \mathrm{~S}_{3} \ldots \ldots \ldots \ldots 1,81$

Aramayoite, $\mathrm{Ag}_{2} \mathrm{~S}$.(Sb,Bi) ${ }_{2} \mathrm{~S}_{3} \ldots \ldots$

Benjaminite, $(\mathrm{Cu}, \mathrm{Ag})_{2} \mathrm{~S} .2 \mathrm{PbS} .2 \mathrm{Bi}_{2} \mathrm{~S}_{3} \ldots \ldots$

Bismuth (native), $\mathrm{Bi}$

Bismuthinite, $\mathrm{Bi}_{2} \mathrm{~S}_{3}$

Bismutoplagionite, $5 \mathrm{PbS} .4 \mathrm{Bi}_{2} \mathrm{~S}_{3} \ldots$

Cosalite, $2 \mathrm{PbS} . \mathrm{Bi}_{2} \mathrm{~S}_{3}$

Emplectite, $\mathrm{Cu}_{2} \mathrm{~S} . \mathrm{Bi}_{2} \mathrm{~S}_{3}$

Galenobismutite, $\mathrm{PbS} . \mathrm{Bi}_{2} \mathrm{~S}_{3} \ldots \ldots$

Guanajuatite, $\mathrm{Bi}_{2}(\mathrm{Se}, \mathrm{S})_{3}$

Kallilite, Ni(Sb,Bi)S

Klaprotholite, $3 \mathrm{Cu}_{2} \mathrm{~S} \mathrm{Bi}_{2} \mathrm{~S}_{3} \ldots$

Matildite, $\left(\mathrm{Ag}_{2}, \mathrm{~Pb}\right) \mathrm{S}_{\mathrm{B}} \mathrm{Bi}_{2} \mathrm{~S}_{3} \ldots$

Rezbanyite, $2 \mathrm{PbS}_{3} 3 \mathrm{Bi}_{2} \mathrm{~S}_{3}$

Schapbachite, $\mathrm{Ag}_{2} \mathrm{~S}$ S.PbS. $\mathrm{Bi}_{2} \mathrm{~S}_{3}$

Tetradymite, $\mathrm{Bi}_{2}(\mathrm{Te}, \mathrm{S})_{3}$

Wittichenite, $3 \mathrm{Cu}_{2} \mathrm{~S} . \mathrm{Bi}_{2} \mathrm{~S}_{8} \ldots$ 
Carrollite, $(\mathrm{Co}, \mathrm{Cu})_{3} \mathrm{~S}_{4}$

Cobsltite, CoAsS

Glaucodot, $(\mathrm{Co}, \mathrm{Fe}) \mathrm{AsS}$

Linnaeite, $\mathrm{Co}_{3} \mathrm{~S}_{4}$

Siegenite, $(\mathrm{Co}, \mathrm{Ni})_{3} \mathrm{~S}_{4} \ldots \ldots \ldots$

Skutterudite, (Co,Ni) $\mathrm{As}_{3}$

Smaltite-chloanthite, (Co,Ni) $\mathrm{As}_{2} \ldots \ldots$

Willyamite, (Co,Ni) SbS _.......... 86, 88, 100, 101

\section{Copper $(\mathbf{C u})$}

Aikinite, $3\left(\mathrm{~Pb}, \mathrm{Cu}_{2}\right) \mathrm{S} \cdot \mathrm{Bi}_{2} \mathrm{~S}_{3}$

$76,78,87,89$

Algodonite, $\mathrm{Cu}_{6} \mathrm{As}$

Benjaminite, $(\mathrm{Cu}, \mathrm{Ag})_{2} \mathrm{~S} .2 \mathrm{PbS} .2 \mathrm{Bi}_{2} \mathrm{~S}_{3} \ldots \ldots$

Berthonite, $2\left(\mathrm{~Pb}, \mathrm{Cu}_{2}\right) \mathrm{S}_{2} \mathrm{Sb}_{2} \mathrm{~S}_{3} \ldots$

Bornite, $\mathrm{Cu}_{5} \mathrm{FeS}_{4}$

Bournonite, $\mathrm{Cu}_{2} \mathrm{~S} .2 \mathrm{PbS} . \mathrm{Sb}_{2} \mathrm{~S}_{3} \ldots \ldots$

Carrollite, $(\mathrm{Co}, \mathrm{Cu})_{3} \mathrm{~S}_{4}$

Chalcocite, $\mathrm{Cu}_{2} \mathrm{~S}$

Chalcopyrite, $\mathrm{Cu}_{2} \mathrm{~S} . \mathrm{Fe}_{2} \mathrm{~S}_{3} \ldots \ldots \ldots \ldots$ 87, 89, 94,95

Chalcostibite, $\mathrm{Cu}_{2} \mathrm{~S}_{\text {S }} \mathrm{Sb}_{2} \mathrm{~S}_{3} \ldots$

Chalmersite. See Cubanite.

Copper (native), Cu $\mathrm{Cu}_{2}$

Covellite, CuS.

Cubanite, CuS.2FeS

Cuprite, $\mathrm{Cu}_{2} \mathrm{O}$

Cuprodescloizite, $\mathrm{Pb}_{2}(\mathrm{Zn}, \mathrm{Cu})_{2} \mathrm{~V}_{2} \mathrm{O}_{9} \cdot \mathrm{H}_{2} \mathrm{O} \ldots$

Delafossite, $\mathrm{CuFeO}_{2} \ldots$

Domeykite, $\mathrm{Cu}_{3} \mathrm{As}$

Emplectite, $\mathrm{Cu}_{2} \mathrm{~S} . \mathrm{Bi}_{2} \mathrm{~S}_{3} \ldots \ldots \ldots$

Enargite, $\mathrm{Cu}_{2} \mathrm{~S} .4 \mathrm{CuS} . \mathrm{As}_{2} \mathrm{~S}_{3} \ldots \ldots \ldots$. 33

Eucairite, $\mathrm{Cu}_{2} \mathrm{Se} . \mathrm{Ag}_{2} \mathrm{Se}$

Famatinite, $\mathrm{Cu}_{2} \mathrm{~S} .4 \mathrm{CuS} . \mathrm{Sb}_{2} \mathrm{~S}_{3} \ldots$

Freibergite, $5(\mathrm{Cu}, \mathrm{Ag})_{2} \mathrm{~S} .2(\mathrm{Cu}, \mathrm{Fe}) \mathrm{S} .2 \mathrm{Sb}_{2} \mathrm{~S}_{3} \ldots \ldots \ldots \ldots, 87$

Germanite, $\mathrm{Cu}_{\mathbf{z}} \mathrm{GeS}_{\mathbf{y}}$

Klaprotholite, $4 \mathrm{Cu}_{2} \mathrm{~S}_{2} \mathrm{Bi}_{2} \mathrm{~S}_{3} \ldots$

Klockmannite, CuSe....... 85

Lengenbachite, 6PbS.(Ag, Cu) ${ }_{2} \mathrm{~S} .2 \mathrm{As}_{2} \mathrm{~S}_{3} \ldots \ldots \ldots$

Luzonite, $\mathrm{Cu}_{2} \mathrm{~S} .4 \mathrm{CuS} . \mathrm{As}_{2} \mathrm{~S}_{3}$ (variety of enargite) $\ldots \ldots \ldots . . . . .53$

Melaconite, $\mathrm{CuO}$ (variety of tenorite)

Rickardite, $\mathrm{Cu}_{4} \mathrm{Te}_{3}$

Seligmannite, $\mathrm{Cu}_{2} \mathrm{~S} .2 \mathrm{PbS} . \mathrm{As}_{2} \mathrm{~S}_{3} \ldots$

Stannite, $\mathrm{Cu}_{2} \mathrm{~S} . \mathrm{FeS} . \mathrm{SnS}_{2} \ldots \ldots$

Stromeyerite, $(\mathrm{Cu}, \mathrm{Ag})_{2} \mathrm{~S}$

Sulvanite, $\mathrm{Cu}_{3} \mathrm{VS}_{4} \ldots$

Tennantite, $5 \mathrm{Cu}_{2} \mathrm{~S} .2(\mathrm{Cu}, \mathrm{Fe}, \mathrm{Zn}) \mathrm{S} .2 \mathrm{As}_{2} \mathrm{~S}_{3} \ldots$

Tenorite, $\mathrm{CuO} \ldots$

Tetrahedrite, $5 \mathrm{Cu}_{2} \mathrm{~S} .2(\mathrm{Cu}, \mathrm{Fe}, \mathrm{Zn}) \mathrm{S} .2 \mathrm{Sb}_{2} \mathrm{~S}_{3} \ldots$

Umangite, $\mathrm{Cu}_{3} \mathrm{Se}_{2} \ldots \ldots$

Weissite, $\mathrm{Cu}_{6} \mathrm{Te}_{3} \ldots \ldots$

Whitneyite, $\mathrm{Cu}_{8} \mathrm{As}$

Wittichenite, $3 \mathrm{Cu}_{2} \mathrm{~S} \cdot \mathrm{Bi}_{2} \mathrm{~S}_{3} \ldots \ldots$ 
Germanium (Ge)

Argyrodite, $4 \mathrm{Ag}_{2} \mathrm{~S} . \mathrm{GeS}_{2}$

Germanite, $\mathrm{Cu}_{\mathbf{x}} \mathrm{GeS}_{\mathbf{y}}$

Gold (Au)

Calaverite, $(\mathrm{Au}, \mathrm{Ag}) \mathrm{Te}_{2}$

Electrum, Ag, Au.

Gold (native), $\mathrm{Au}$

Krennerite, $(\mathrm{Ag}, \mathrm{Au}) \mathrm{Te}_{2}$

Nagyagite, $\mathrm{Pb}, \mathrm{Au}, \mathrm{Te}, \mathrm{S}$ 76,78

Petzite, (Ag, Au $)_{2} \mathrm{Te}_{2}$

Sylvanite, $(\mathrm{Ag}, \mathrm{Au}) \mathrm{Te}_{2}$

\section{Iron $(\mathbf{F e})$}

Arsenoferrite, FeAs_.

Arsenopyrite, FeAsS

Berthierite, FeS.Sb ${ }_{2} \mathrm{~S}_{3} \ldots \ldots$

Bornite, $\mathrm{Cu}_{5} \mathrm{FeS}_{4}$

Bravoite, (Fe,Ni) $\mathrm{S}_{2} \ldots$

Chalcopyrite, $\mathrm{Cu}_{2} \mathrm{~S} . \mathrm{Fe}_{2} \mathrm{~S}_{3} \ldots \ldots$

Chalmersite. See Cubanite.

Chromite, $\mathrm{FeO} . \mathrm{Cr}_{2} \mathrm{O}_{3} \ldots$

Cubanite, CuS.2FeS.

Delafossite, $\mathrm{CuFe}_{2} \mathrm{O}_{2}$

Ferberite, $\mathrm{FeWO}_{4}$

Franklinite, (Fe, $\mathrm{Zn}, \mathrm{Mn}) \mathrm{O} .(\mathrm{Fe}, \mathrm{Mn})_{2} \mathrm{O}_{3} \ldots \ldots 1,102$

Freibergite, $\mathbf{5}(\mathrm{Cu}, \mathrm{Ag})_{2} \mathrm{~S} .2(\mathrm{Cu}, \mathrm{Fe}) \mathrm{S} .2 \mathrm{Sb}_{2} \mathrm{~S}_{3} \ldots \ldots . .867$

Glaucodot, $(\mathrm{Co}, \mathrm{Fe}) \mathrm{AsS}$

Goethite, $\mathrm{Fe}_{2} \mathrm{O}_{3} \cdot \mathrm{H}_{2} \mathrm{O} \ldots \ldots$

Hematite, $\mathrm{Fe}_{2} \mathrm{O}_{3}$

Ilmenite, $\mathrm{FeTiO}_{3} \ldots$

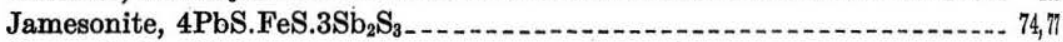

Leucopyrite, $\mathrm{Fe}_{3} \mathrm{As}_{4} \ldots$

Löllingite, $\mathrm{FeAs}_{2} \ldots$

Magnetite, $\mathrm{Fe}_{3} \mathrm{O}_{4} \ldots \ldots$

Marcasite, $\mathrm{FeS}_{2}$

Pentlandite, (Ni,Fe)S............ 88

Plessite, (Ni,Fe)AsS

Pyrite, $\mathrm{FeS}_{2} \ldots \ldots$

Pyrrhotite, $\mathrm{FeS}_{1+}$

Stannite, $\mathrm{Cu}_{2} \mathrm{~S} . \mathrm{FeS} . \mathrm{SnS}_{2} \ldots \ldots$

Sternbergite, $\mathrm{AgFe}_{2} \mathrm{~S}_{3}$

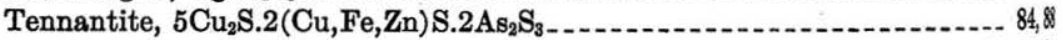

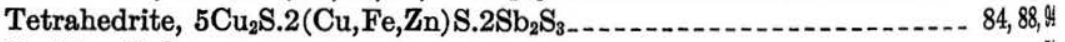

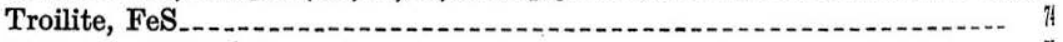

Violarite, $(\mathrm{Ni}, \mathrm{Fe})_{3} \mathrm{~S}_{4}$

Wolframite, $(\mathrm{Fe}, \mathrm{Mn}) \mathrm{WO}_{4} \ldots \ldots$

\section{Lead $(\mathbf{P b})$}

Aikinite, $3\left(\mathrm{~Pb}, \mathrm{Cu}_{2}\right) \mathrm{S} . \mathrm{Bi}_{3} \mathrm{~S}_{3} \ldots \ldots \ldots$. $\ldots 1,87,88$

Alaskaite, $\mathrm{Ag}_{2} \mathrm{~S} . \mathrm{PbS} \mathrm{Bi}_{2} \mathrm{~S}_{3}$

Altaite, PbTe....... is

Andorite, AgS.2PbS.3Sb ${ }_{2} \mathrm{~S}_{3} \ldots$

Baumhauerite, $4 \mathrm{PbS} .3 \mathrm{As}_{2} \mathrm{~S}_{3} \ldots \ldots \ldots$ it 
Benjaminite, $(\mathrm{Cu}, \mathrm{Ag})_{2} \mathrm{~S} .2 \mathrm{PbS} .2 \mathrm{Bi}_{2} \mathrm{~S}_{3} \ldots$

Berthonite, 2( $\left.\mathrm{PbCu}_{2}\right) \mathrm{S}_{2} \mathrm{Sb}_{2} \mathrm{~S}_{3}$

Bismutoplagionite, $5 \mathrm{PbS} .4 \mathrm{Bi}_{2} \mathrm{~S}_{3}$

Boulangerite, $5 \mathrm{PbS} .2 \mathrm{Sb}_{2} \mathrm{~S}_{3}$

Bournonite, $\mathrm{Cu}_{2} \mathrm{~S} .2 \mathrm{PbS} . \mathrm{Sb}_{2} \mathrm{~S}_{3} \ldots$

Brongniardite, $\mathrm{Ag}_{2} \mathrm{~S} . \mathrm{PbS} . \mathrm{Sb}_{2} \mathrm{~S}_{3} \ldots$

Clausthalite, $\mathrm{PbSe}$

Cosalite, 2PbS.Bi $\mathrm{S}_{3}$

Cuprodescloizite, $\mathrm{Pb},(\mathrm{Zn}, \mathrm{Cu})_{2} \mathrm{~V}_{2} \mathrm{O}_{9} \cdot \mathrm{H}_{2} \mathrm{O}$

Cylindrite, $6 \mathrm{PbS} \mathrm{Sb}_{2} \mathrm{~S}_{3} .6 \mathrm{SnS}_{2} \ldots$

Dufrenoysite, $2 \mathrm{PbS} . \mathrm{As}_{2} \mathrm{~S}_{3} \ldots \ldots \ldots$

Franckeite, $5 \mathrm{PbS} . \mathrm{Sb}_{2} \mathrm{~S}_{3}, 2 \mathrm{SnS}_{2} \ldots$

Freieslebenite, $3 \mathrm{Ag}_{2} \mathrm{~S} .4 \mathrm{PbS} .8 \mathrm{Sb}_{2} \mathrm{~S}_{3} \ldots$

Galena, PbS.

Galenobismutite, $\mathrm{PbS} . \mathrm{Bi}_{2} \mathrm{~S}_{3} \ldots$

Geocronite, $5 \mathrm{PbS} . \mathrm{Sb}_{2} \mathrm{~S}_{3}$

Guitermanite, $3 \mathrm{PbS} . \mathrm{As}_{2} \mathrm{~S}_{3} \ldots$

Jamesonite, $4 \mathrm{PbS} . \mathrm{FeS} .3 \mathrm{Sb}_{2} \mathrm{~S}_{3} \ldots$

Jordanite, $4 \mathrm{PbS} . \mathrm{As}_{2} \mathrm{~S}_{3} \ldots$

Keeleyite, $\mathrm{PbS} . \mathrm{Sb}_{2} \mathrm{~S}_{3}$

Legenbachite, $6 \mathrm{PbS} .(\mathrm{Ag}, \mathrm{Cu})_{2} \mathrm{~S} .2 \mathrm{As}_{2} \mathrm{~S}_{3}$

Matildite, $\left(\mathrm{Ag}_{2}, \mathrm{~Pb}\right) \mathrm{S} . \mathrm{Bi}_{2} \mathrm{~S}_{3} \ldots$

Meneghinite, $4 \mathrm{PbS} . \mathrm{Sb}_{2} \mathrm{~S}_{3}$

Nagyagite, $\mathrm{Pb}, \mathrm{Au}, \mathrm{Te}, \mathrm{S}$

Naumannite, $\left(\mathrm{Ag}_{2}, \mathrm{~Pb}\right) \mathrm{Se}_{\ldots}$

0wyheeite, $8 \mathrm{PbS} .2 \mathrm{Ag}_{2} \mathrm{~S} .5 \mathrm{Sb}_{2} \mathrm{~S}_{3} \ldots \ldots \ldots . .82$

Plagionite, $5 \mathrm{PbS} .4 \mathrm{Sb}_{2} \mathrm{~S}_{3} \ldots$

Rathite, $3 \mathrm{PbS} .2 \mathrm{As}_{2} \mathrm{~S}_{3} \ldots$

Rezbanyite, $2 \mathrm{PbS}_{3} 3 \mathrm{Bi}_{2} \mathrm{~S}_{3}$

Schapbachite, $\mathrm{Ag}_{2} \mathrm{~S} . \mathrm{PbS} . \mathrm{Bi}_{2} \mathrm{~S}_{3}$

Seligmannite, $\mathrm{Cu}_{2} \mathrm{~S} .2 \mathrm{PbS} . \mathrm{As}_{2} \mathrm{~S}_{3}$

Semseyite, $9 \mathrm{PbS} .4 \mathrm{Sb}_{2} \mathrm{~S}_{3}$

Teallite, SnS.PbS _

Uraninite, uranate of $\mathrm{U}, \mathrm{Pb}$, etc

Zinkenite, $\mathrm{PbS} . \mathrm{Sb}_{2} \mathrm{~S}_{3}$

\section{Manganese (Mn)}

Alabandite, MnS.....

Braunite, $3 \mathrm{Mn}_{2} \mathrm{O}_{3} \cdot \mathrm{MnSiO}_{2}$

Chalcophanite, $(\mathrm{Mn}, \mathrm{Zn}) 0.2 \mathrm{MnO}_{2} \cdot 2 \mathrm{H}_{2} \mathrm{O} \ldots$

Franklinite; $(\mathrm{Fe}, \mathrm{Zn}, \mathrm{Mn})$ O. $(\mathrm{Fe}, \mathrm{Mn})_{2} \mathrm{O}_{3} \ldots \ldots 101$

Hauerite, $\mathrm{MnS}_{2} \ldots \ldots \ldots$

Hausmannite, $\mathrm{Mn}_{3} \mathrm{O}_{4}$

Hübnerite, $\mathrm{MnWO}_{4}$

Manganite, $\mathrm{Mn}_{2} \mathrm{O}_{3}$

Polianite, $\mathrm{MnO}_{2} \ldots$

Psilomelane, $\mathrm{Mn}_{2} \mathrm{MnO}_{5}$

Pyrolusite, $\mathrm{MnO}_{2} \ldots 9$

Wolframite, $(\mathrm{Fe}, \mathrm{Mn}) \mathrm{WO}_{4}$

Mercury (Hg)

Cinnabar, $\mathrm{HgS}$

Coloradoite, $\mathrm{HgTe}$ 
Livingstonite, $\mathrm{HgS.} 2 \mathrm{Sb}_{2} \mathrm{~S}_{3}$

Tiemannite, HgSe

Molybdenum (Mo)

Molybdenite, $\mathrm{MoS}_{2}$

Nickel (Ni)

Bravoite, (Fe,Ni) $\mathrm{S}_{2 \ldots}$

Breithauptite, NiSb. . .

Chloanthite-smaltite, $(\mathrm{Ni}, \mathrm{Co}) \mathrm{As}_{2} \ldots \ldots . . .197$

Corynite, Ni(As,Sb)S... 97, 101

Gersdorffite, NiAsS

Kallilite, $\mathrm{Ni}(\mathrm{Sb}, \mathrm{Bi}) \mathrm{S} \ldots \ldots \ldots$.

Maucherite, $\mathrm{Ni}_{3} \mathrm{As}_{2} \ldots \ldots, 98,98$

Melonite, $\mathrm{Ni}_{2} \mathrm{Te}_{3}$

Millerite, NiS_..._-_._. 89

Niccolite, NiAs.

Pentlandite, $(\mathrm{Ni}, \mathrm{Fe}) \mathrm{S} \ldots \ldots$

Plessite, (Ni,Fe)AsS

Polydymite, $\mathrm{Ni}_{3} \mathrm{~S}_{4}$

Rammelsbergite, $\mathrm{NiAs}_{2}$

Siegenite, $(\mathrm{Co}, \mathrm{Ni})_{3} \mathrm{~S}_{4}$

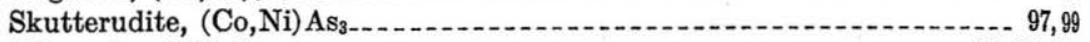

Ullmanite, NiSbS_ _.

Violarite, $(\mathrm{Ni}, \mathrm{Fe})_{3} \mathrm{~S}_{4} \ldots$

Willyamite, (Co,Ni)SbS_............. 86, 88, 100, 101

Platinum (Pt)

Sperrylite, $\mathrm{PtAs}_{2}$

Selenium (Se)

Aguilarite, $\mathrm{Ag}_{2}(\mathrm{Se}, \mathrm{S}) \ldots \ldots . . . .60,86$

Clausthalite, PbSe_...

Eucairite, $\mathrm{Cu}_{2} \mathrm{Se} . \mathrm{Ag}_{2} \mathrm{Se}$

Guanajuatite, $\mathrm{Bi}_{2}(\mathrm{Se}, \mathrm{S})_{3} \ldots$

Klockmannite, CuSe_....... 85

Naumannite, $\left(\mathrm{Ag}_{2}, \mathrm{~Pb}\right) \mathrm{Se} \ldots \ldots \ldots$

Tiemannite, HgSe... 93, 94

Umangite, $\mathrm{Cu}_{3} \mathrm{Se}_{2} \ldots \ldots$

Silver (Ag)

Aguilarite, $\mathrm{Ag}_{2}(\mathrm{Se}, \mathrm{S}) \ldots$

Alaskaite, $\mathrm{Ag}_{2} \mathrm{~S}$.PbS. $\mathrm{Bi}_{2} \mathrm{~S}_{3} \ldots$

Andorite, $\mathrm{Ag}_{2} \mathrm{~S} .2 \mathrm{PbS} .3 \mathrm{Sb}_{2} \mathrm{~S}_{3} \ldots \ldots \ldots \ldots 1,94$

Aramayoite, $\mathrm{Ag}_{2} \mathrm{~S} .(\mathrm{Sb}, \mathrm{Bi})_{2} \mathrm{~S}_{3} \ldots \ldots \ldots 8$

Argentite, $\mathrm{Ag}_{2} \mathrm{~S}$

Argyrodite, $4 \mathrm{Ag}_{2}$ S.GeS $\mathrm{G}_{2} \ldots \ldots \ldots$.

Benjaminite, $(\mathrm{Cu}, \mathrm{Ag})_{2} \mathrm{~S} .2 \mathrm{PbS} .2 \mathrm{Bi}_{2} \mathrm{~S}_{3} \ldots \ldots \ldots$

Brongniardite, $\mathrm{Ag}_{2} \mathrm{~S} . \mathrm{PbS} . \mathrm{Sb}_{2} \mathrm{~S}_{3}$

Calaverite, $(\mathrm{Au}, \mathrm{Ag}) \mathrm{Te}_{2}$

Canfieldite, $4 \mathrm{Ag}_{2} \mathrm{~S} . \mathrm{SnS}_{2}$ with some germanium

Cerargyrite, $\mathrm{AgCl}_{\mathrm{C}} \ldots$

Dyscrasite, $\mathrm{Ag}_{3} \mathrm{Sb}_{2}$

Electrum, $\mathrm{Ag}, \mathrm{Au}$ 
Eucairite, $\mathrm{Cu}_{2} \mathrm{Se} . \mathrm{Ag}_{2} \mathrm{Se}$

Freibergite, $5(\mathrm{Cu}, \mathrm{Ag})_{2} \mathrm{~S} .2(\mathrm{Cu}, \mathrm{Fe}) \mathrm{S} .2 \mathrm{Sb}_{2} \mathrm{~S}_{3} \ldots \ldots$

Freieslebenite, $3 \mathrm{Ag}_{2} \mathrm{~S} .4 \mathrm{PbS} .3 \mathrm{Sb}_{2} \mathrm{~S}_{3} \ldots \ldots$

Hessite, $\mathrm{Ag}_{2} \mathrm{Te}$

Huntilite, $\mathrm{Ag}_{3} \mathrm{As}$

Krennerite, (Ag, $\mathrm{Au}) \mathrm{Te}_{2} \ldots \ldots$

Lengenbachite, 6PbS.(Ag, Cu) ${ }_{2} \mathrm{~S} . \mathrm{As}_{2} \mathrm{~S}_{3} \ldots \ldots$

Matildite, $\left(\mathrm{Ag}_{2}, \mathrm{~Pb}\right) \mathrm{S} . \mathrm{Bi}_{2} \mathrm{~S}_{3}$

Miargyrite, $\mathrm{Ag}_{2} \mathrm{~S}_{\mathrm{S}} \mathrm{Sb}_{2} \mathrm{~S}_{3} \ldots \ldots$

Naumannite, $\left(\mathrm{Ag}_{2}, \mathrm{~Pb}\right) \mathrm{Se}_{\ldots} \ldots$

0wyheeite, $8 \mathrm{PbS} .2 \mathrm{Ag}_{2} \mathrm{~S} .5 \mathrm{Sb}_{2} \mathrm{~S}_{3} \ldots$

Pearcite, $8 \mathrm{Ag}_{2} \mathrm{~S} . \mathrm{As}_{2} \mathrm{~S}_{3} \ldots$

Petzite, $(\mathrm{Ag}, \mathrm{Au})_{2} \mathrm{Te}$

Polybasite, $8 \mathrm{Ag}_{2} \mathrm{~S}_{\mathrm{S}} \mathrm{Sb}_{2} \mathrm{~S}_{3} \ldots \ldots$

Proustite, $3 \mathrm{Ag}_{2} \mathrm{~S}$. $\mathrm{As}_{2} \mathrm{~S}_{3} \ldots$

Pyrargyrite, $3 \mathrm{Ag}_{2} \mathrm{~S}_{\mathrm{S}} \mathrm{Sb}_{2} \mathrm{~S}_{3} \ldots \ldots$

Schapbachite, $\mathrm{Ag}_{2} \mathrm{~S} . \mathrm{PbS} \cdot \mathrm{Bi}_{2} \mathrm{~S}_{3} \ldots \ldots$

Silver (native), Ag _.

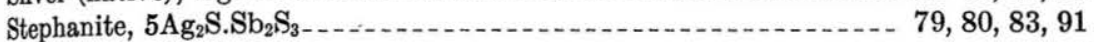

Sternbergite, $\mathrm{AgFe}_{2} \mathrm{~S}_{3} \ldots \ldots$

Stromeyerite, $(\mathrm{Cu}, \mathrm{Ag})_{2} \mathrm{~S} \ldots$

Sylvanite, $(\mathrm{Ag}, \mathrm{Au}) \mathrm{Te}_{2} \ldots \ldots$

Tellurium (Te)

Altaite, $\mathrm{PbTe}$

Calaverite, (Au, $\mathrm{Ag}) \mathrm{Te}_{2} \ldots \ldots \ldots \ldots$ 76, 78

Coloradoite, HgTe...

Hessite, $\mathrm{Ag}_{2} \mathrm{Te}$

Krennerite, $(\mathrm{Ag}, \mathrm{Au}) \mathrm{Te}_{2} \ldots \ldots$

Melonite, $\mathrm{Ni}_{2} \mathrm{Te}_{3}$

Nagyagite, Pb, Au, Te,S _.

Petzite, $(\mathrm{Ag}, \mathrm{Au})_{2} \mathrm{Te}_{\mathrm{N}}$

Rickardite, $\mathrm{Cu}_{4} \mathrm{Te}_{3}($ ?)

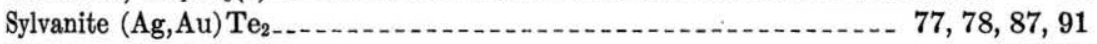

Tellurium (native), Te_...

Tetradymite, $\mathrm{Bi}_{2}(\mathrm{Te}, \mathrm{S})_{3} \ldots \ldots \ldots$ 73, 76

Weissite, $\mathrm{Cu}_{5} \mathrm{Te}_{3}($ ?)

Thallium (TI)

Lorandite, $\mathrm{Tl}_{2} \mathrm{~S} . \mathrm{As}_{2} \mathrm{~S}_{3}$

Vrbaite, $\mathrm{Tl}_{2} \mathrm{~S} .3(\mathrm{As}, \mathrm{Sb})_{2} \mathrm{~S}_{3}$

Tin (Sn)

Canfieldite, $4 \mathrm{Ag}_{2} \mathrm{~S} . \mathrm{SnS}_{2}$ with some germanium $\ldots$.

Cassiterite, $\mathrm{SnO}_{2}$

Cylindrite, $6 \mathrm{PbS} \mathrm{Sb}_{2} \mathrm{~S}_{3} .6 \mathrm{SnS}_{2} \ldots \ldots \ldots, 81,88$

Franckeite, $5 \mathrm{PbS} \mathrm{Sb}_{2} \mathrm{~S}_{3} .2 \mathrm{SnS}_{2}$

Stannite, $\mathrm{Cu}_{2}$ S.FeS.SnS ${ }_{2} \ldots$

Teallite, SnS.PbS

Titanium (Ti)

Ilmenite, $\mathrm{FeTiO}_{3}$

Rutile, $\mathrm{TiO}_{2}$ 
Tungsten (W)

Ferberite, $\mathrm{FeWO}_{4}$

Hübnerite, $\mathrm{MnWO}$

Tungstenite, $\mathrm{WS}_{2}$

Wolframite, $(\mathrm{Fe}, \mathrm{Mn}) \mathrm{WO}_{4}$

\section{Uranium (U)}

Uraninite, uranate of $\mathrm{U}, \mathrm{Pb}$, etc

Vanadium (V)

Cuprodescloizite, $\mathrm{Pb}_{2}(\mathrm{Zn}, \mathrm{Cu})_{2} \mathrm{~V}_{2} \mathrm{O}_{9} \cdot \mathrm{H}_{2} \mathrm{O}$

Sulvanite, $\mathrm{Cu}_{3} \mathrm{VS}_{4}$

\section{Zine (Zn)}

Chalcophanite, $(\mathrm{Mn}, \mathrm{Zn}) \mathrm{O} .2 \mathrm{MnO}_{2} .2 \mathrm{H}_{2} \mathrm{O}$

Cuprodescloizite, $\mathrm{Pb}_{2}(\mathrm{Zn}, \mathrm{Cu})_{2} \mathrm{~V}_{2} \mathrm{O}_{9} \cdot \mathrm{H}_{2} \mathrm{O}$

Franklinite, $(\mathrm{Fe}, \mathrm{Zn}, \mathrm{Mn}) \mathrm{O} .(\mathrm{Fe}, \mathrm{Mn})_{2} \mathrm{O}_{3}$

Sphalerite, $\mathrm{ZnS}$

Tetrahedrite, $5 \mathrm{Cu}_{2} \mathrm{~S} .2(\mathrm{Cu}, \mathrm{Fe}, \mathrm{Zn}) \mathrm{S} .2 \mathrm{Sb}_{2} \mathrm{~S}_{3}$

Voltzite, $4 \mathrm{ZnS} . \mathrm{ZnO}$

Wurtzite, $\mathrm{ZnS}$ 


\section{Part 4. MICROCHEMICAL METHODS}

\section{INTRODUCTION}

Microchemical qualitative analysis consists in testing for one or more unknown elements in a solution by adding suitable reagents that will precipitate these elements, if present, out of the unknown solution. The reactions are carried out on a glass slide and observed under the microscope. The reagents usually chosen are those that will precipitate the elements as crystals of characteristic shape and color; the crystals are thus diagnostic of the elements sought. With only a few elements is the precipitate amorphous, and this is only because suitable crystalline precipitates are lacking. In the thiocyanate test for iron no precipitate is thrown down, but the unknown solution turns a deep-red color when the reagent is added.

Two great advantages possessed by microchemical analysis over ordinary qualitative analysis are the saving of time and the saving of material. Practically every microchemical test can be made in 10 minutes or even less, whereas an ordinary qualitative analysis may consume hours in evaporations over a steam bath or in filtrations. Microchemical analyses also require much less material than ordinary chemical analyses. A microchemical analysis can usually be made on a quantity of material almost invisible to the unaided eye.

Microchemical methods are by no means new or modern, but they have been singularly neglected by the analytical chemist, owing to the prevailing belief that microscopic methods are intricate and require a long apprenticeship. They have received far greater recognition from European than from American chemists and petrologists.

The pioneer investigations in microchemistry were carried out by Boricky, ${ }^{53}$ who worked out methods for determining the elements in rock-forming minerals.

The methods used by Boričky were improved and expanded by Prof. H. Behrens, of the Polytechnic Sichool of Delft, Holland, who developed microchemical methods for the identification of practically all the then known solid elements. ${ }^{54}$ His book was later revised

\footnotetext{
"Borlčky, Emmanuel, Elemente einer neuen chemische-mikroskopischen Mineral- und Gesteins-Analyse: Naturwiss. Landesdurchf. Böhmen Archiv, Band 3, Prague, 1877. Translated into English by Winchell, N. H., Minnesota Geol. Survey Ann. Rept., vol. 19, pp. $1-80,1892$.

"Behrens, H., Mikrochemische Methoden, Amsterdam, 1882.
} 
and translated into English by himself. ${ }^{55}$ Behrens deserves the credit of having done more to further the science of microchemistry than anyone else. Several editions of his textbook have appeared, and the latest, in 1921, revised by Kley, ${ }^{56}$ is the foremost work on this subject in Europe.

The best text written in English is that of Chamot, ${ }^{57}$ who, with his coworkers at Cornell University, has carried on instruction in microchemistry for nearly 30 years. For many years Cornell was the only institution in the United States where microchemistry was taught. Recently other institutions have taken it up, but it is regrettable that many of the leading universities still offer no courses in this subject.

Credit for the first application of microchemical methods to the identification of opaque minerals in polished sections is due to Prof. Waldemar Lindgren, of the Massachusetts Institute of Technology, who initiated instruction in this application of the method in 1920. The present writer first became acquainted with microchemical methods while studying under Professor Lindgren in 1922. The possibilities of the method are set forth in a timely editorial. ${ }^{58}$

Recently McKinstry ${ }^{59}$ has contributed a valuable article, refer. ences from which will be found in the description of the tests for cobalt (p. 137) and elsewhere in this bulletin.

A valuable series of articles on microchemical methods, by Put. nam, Roberts, and Selchow ${ }^{60}$ has recently appeared. These authors record the results of much new and original research work and give tests for some of the rarer elements. The present writer is indebted to them for the pyridine double bromide test for gold that appears on page 153 .

\section{PROCEDURE AND APPARATUS}

The elements of a mineral are identified by means of microchemical tests made on a very small amount of material gouged out of the surface of the mineral. In order to be certain that the material tested is pure-that is, does not contain extraneous minerals which may be intergrown with the mineral to be tested-a polished surface is prepared and observed under a reflecting microscope, and the powder to be tested is gouged from this surface by means of a chisel-pointed needle. The powder thus obtained is collected by

${ }^{55}$ Behrens, H., A manual of microchemical analysis, London, 1894. Now out of print

${ }^{56}$ Behrens, H., Mikrochemische Analyse, revised by P. D. C. Kley, Leipzig, Leopold Voss 1921. No translation into English has yet appeared.

${ }^{67}$ Chamot, E. M., Elementary chen:ical microscopy, New York, 1921.

${ }^{6}$ Lindgren, Waldemar, Microchemical methods; Econ. Geology, vol. 19, p. 762, 1924.

${ }^{59}$ McKinstry, H. E., Microchemical methods in the identification of opaque mineras; Econ. Geology, vol. 22, p. 830, 1927.

${ }^{00}$ Putnam, P. C., Roberts, E. J., and Selchow, D. H., Contributions to determinat: mineralogy ; Am. Jour. Sci., 5th ser., vol. 15, pp. 89-102, 253-261, 423-430, 455-460,1928 
means of a sharpened match or splinter of soft wood, the point of which has been moistened and wiped nearly dry. The powder adheres to the damp wood. The match with its adhering powder is dipped into a drop of nitric acid or other suitable acid on a glass slide. The surface tension which holds the powder to the wood is broken, and the powder drops from the wood and falls to the bottom of the drop. The acid is evaporated off by holding the slide over a microflame. ${ }^{61}$ The residue is observed under the microscope to see if any of the original material has remained unattacked. If necessary, successive drops of the reagent can be applied and evaporated off until the material has been dissolved or broken up chemically. The residue is tested by means of microchemical tests. In general, this method can be applied to any mineral, but it is most suitable to the opaque or nearly opaque ore minerals, because nearly all of these can be taken into solution by means of ordinary acids. The quantity of material tested depends on the delicacy of the test, but usually an amount which can be collected within an area on the polished surface from 0.17 to 0.35 millimeter in diameter is sufficient. The present writer has hitherto rejected tests which have called for an area of pure mineral greater than the larger figure. The textbooks used in this work are those of Chamot and of Behrens and Kley, already referred to. Neither was written primarily with the idea of adapting the methods to mineralogy, but the methods described in both are for the most part applicable to these tests. Chamot describes in great detail the effect that some interfering elements have on the tests. However, his descriptions do not exhaust the combinations of elements found in ore minerals. His work is open to the objection that many elements, some of great importance in the ore minerals-gold, for instance-are not even mentioned. Likewise no tests are given for tellurium, selenium, molybdenum, vanadium, tungsten, and the rarer elements. Behrens and Kley's book is written in German. It gives tests for practically every element found in ore minerals but is open to the objection that the effect of interfering elements is hardly mentioned. For instance, the thallium nitrate test for gold is given the preference over other tests. ${ }^{62}$ It is a very beautiful and delicate test when applied to pure native gold but is valueless in the presence of tellurium ions.

The efforts of the present writer have been directed to the determination of the extent to which a test for an element in a mineral is interfered with by chemical compounds of other elements occurring in the same mineral. He has found, for instance, that the presence

\footnotetext{
"This method of transferring material from a polished section to a glass slide was originated by Dr. K. K. Landes while a student in the laboratory of economic geology at Harvari University.

"Behrens, H., op. cit. (1921), p. 155.
} $15122-31-9$ 
of copper ions does not interfere with the precipitation of silver as a bichromate, whereas lead is precipitated as bichromate simulta. neously with silver bichromate and somewhat masks the recognition of the silver compound. Consequently, if lead is greatly in excess of silver it must be removed from the solution before proceeding with the silver test. In other tests a double compound of the interfering elements may be formed. For example, lead is usually precipitated as an iodide. This is honey-yellow in color and crystallizes in welldefined plates. But if mere traces of bismuth ions are present in solution, the lead precipitate is entirely changed in character, and a rusty-brown amorphous precipitate results.

These interfering effects have made it necessary to experiment on each mineral until a suitable procedure could be developed to obviate the difficulties encountered. Usually a chemical separation is necessary. One or more of the elements in the drop is precipitated, learing the other element or elements in solution. Methods of filtration on a microscopic scale were tried out, especially the Behrens method described by Chamot. ${ }^{63}$ These methods were found unsuitable owing to the small size of the drops used in the present investigation, the amount of liquid absorbed by the filter paper being too great. A suitable method of filtration was devised, involving the use of small capillary tubes. The precipitate in the drop is allowed to settle; water is carefully added to the drop in case the evaporation is rapid. Usually the precipitate clings to the glass slide after it has settled. The capillary tube is dipped into the drop and the filtrate is drawn up into the tube by capillarity. The presence of a few particles of the precipitate in the liquid can be neglected. No difficulties from this source have been encountered by the writer. The end of the tube is then moved to another place on the slide, and the liquid is removed by blowing on the other end of the tube. The lower end of the tube should be in contact with the slide before blowing the drop out of the tube, otherwise the drop tends to cling to the outside of the tube.

The precipitate is then washed with one or more drops of wate: or dilute acid to remove the remaining traces of the filtrate. Each drop is picked up by means of the capillary tube and either added to the filtrate or blown out onto a blotter and rejected.

Collecting the material.-The polished section is placed in the fild of the microscope and observed, using a short-mounted Bausch \& Lomb 16-millimeter or Leitz No. 3 objective. Objectives of higher magnification focus too close to the surface of the specimen to permit the free manipulation of the needle.

An area of the mineral to be tested is then selected. It should be free from small inclusions of extraneous minerals. The diameter of

* Chamot. E. M., op. cit., p. 286. 
the mineral should be a little larger than the minimum diameter required to give a satisfactory test, as it requires considerable skill to avoid pushing the needle over the mineral boundary.

The size of needle depends chiefly on the hardness of the mineral scratched. The needles used by the author are manufactured $b_{\bar{y}}$ R. J. Roberts, Redditch, England, and range from Sharps No. 6 to No. 12 in size. With the smaller sizes a smaller area can be scratched. The No. 12 needles are very easily broken, however, and can be used only on very soft minerals. For harder minerals, such as cobaltite, the force necessary to obtain a groove requires a larger and stronger needle, and No. 6 has been found satisfactory.

The point is ground off by holding the needle diagonally against a rapidly rotating carborundum wheel. The surface obtained is a frustum of a cone. The contact between needle and wheel should be only momentary, a fraction of a second, as it is not necessary to grind off entirely the taper-

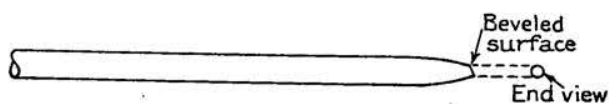

FIgURE 11.-Enlarged view of needle for scraping polished surface ing point of the needle. The beveled surface should make an angle of about $60^{\circ}$ with the axis of the needle. (See fig. 11.)

The needle is then brought into the field of the microscope and the point is worked back and forth within the area until a groove or crater-like cavity is gouged. The depth of this cavity is usually kept considerably less than the diameter of its intersection with the surface of the section-a precaution necessary to avoid penetration into an adjoining underlying mineral.

Some difficulty is usually experienced in scratching the harder minerals. It is best to follow a small pit, seam, or abrasion on the surface. The needle wears rapidly and should be sharpened after every operation. In the softer minerals a rolling motion while pushing the needle is sometimes of help.

A sharpened match is highly effective in collecting the powder. A jagged point is to be avoided. The point should be smooth and regular in shape. A safety-razor blade has been found superior to a knife in sharpening the match. After wetting the end of the match, it should be wiped with a clean rag to remove the superfluous moisture before touching it to the specimen. If it is too wet, some liquid is likely to be transferred from the match to the specimen. A little practice will indicate the extent to which the moisture should be removed.

A platinum wire, in which a loop has been bent at its lower end, is the best instrument for applying the drops of the necessary reagents to the slide. The size of the drop should be kept to a minimum, and the deeper the drop with respect to its diameter on the slide the 
slower will be the rate of evaporation and the larger the growth of the crystals to be investigated. A wire of very small diameter bends too readily and is hard to keep in shape. A wire of comparatively large diameter does not readily permit the bending of a small loop. The diameter of the wire used by the author is 0.35 millimeter, and the inside diameter of the loop is 0.62 millimeter. The loop is formed by bending around a needle.

Glass slides.-The chemical operations are carried out on a glass slide. The slide should be perfectly clean and free from scratches or abrasions. The slides used in mounting thin sections for petro. graphic work are satisfactory. Those used in this work are 25 by 45 millimeters in area and not over 1 millimeter thick. If heat is to be applied, the drop should be placed at one corner of the slide, otherwise the slide will almost certainly break.

Keeping a slide clean is much more difficult than would appear at first thought. The slightest trace of grease or other material on the slide causes the drop to spread and prevents certain manipulations necessary for the best performance of the tests. The usual sulphuric chromic cleansing mixture is satisfactory for cleaning slides. The writer uses a soap containing pumice. The soap is rubbed with a small wet sponge until the sponge is partly filled with lather. The slide is first rubbed with the soapy sponge, then held under a faucet supplying water as hot as can be comfortably endured. When the lather is completely removed by the hot water, the slide is dipped into a vessel containing distilled water in order to remove the remaining tap water. It is then removed, shaken to remove as much of the distilled water as possible, and stood up on end to dry.

The upper end of the slide should lean against a clean, smooth, vertical surface, and the lower end should rest on a paper towel of filter to remove the water as it collects at the bottom of the slide. This procedure obviates the necessity of drying the slide with a cloth, which might add lint or a film of grease to the slide. The clean slides are kept in a beaker and protected from dust by a watch. glass cover.

The tests given by Chamot ${ }^{84}$ illustrate the difficulty in getting a perfectly clean slide. The extreme cleanliness required by the use of the ultramicroscope is not necessary, but the slide should be suff. ciently clean to permit the performance of Chamot's method I. (See p. 124.) The following test is sufficient to determine this matter of cleanliness: Dip a clean platinum wire into a drop of water placed on the slide and draw the wire slowly out of the drop, keeping the end in contact with the surface of the slide. If a channel of liquid accompanies the wire, the slide is clean, for otherwise the liquid will not follow the wire.

es Chamot, E. M., op. cit., p. 150. 
Microburner.-The ordinary Bunsen flame is not suitable for microchemical work, as it spreads the heat over too great an area. The somewhat elaborate auxiliary flame described by Chamot ${ }^{65}$ is likewise not essential. An improvised alcohol lamp made by the writer (fig. 12) is just as good and obviates the necessity of a gas connection. The temperature attained by the flame need not be much above the boiling point of the acids used. Chemical reactions involving roasting or sublimation have not been utilized in the present investigation; all tests used are wet tests. The principal requirement of the lamp is that it furnish a flame of small cross section.

Reagents.-A great deal more difficulty is experienced in getting a mineral in solution by evaporating a drop of acid containing the mineral on a glass slide than by placing the mineral in a test tube containing the reagent and boiling the reagent a minute or two. When heating the drop on a slide, a good part of the reagent is evaporated before it comes to boiling, and even after it reaches the boiling point it is entirely evaporated within a few seconds at the most. For this reason it is essential to have a drop of minimum

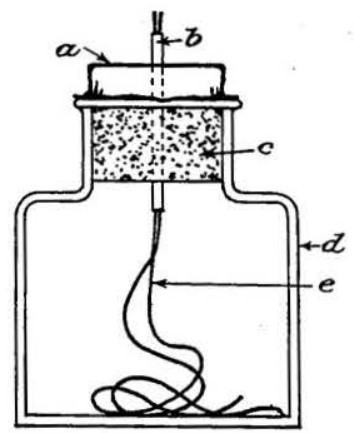

F I G U R D 12. - Improvised alcohol lamp. $a$, Tinfoil cover to keep cork from catching fire; $b$, brass tube; $c$, cork; $d$, ink bottle; $e$, one or two strings

diameter and maximum thickness rather than to have it spread out over the slide.

Very few of the ore minerals thus far tested have hitherto resisted attack by the reagents used. Some oxides, such as chromite, hematite, and cassiterite, can not be brought into solution by nitric acid on a slide. Some sulphides, sulpharsenites, and sulphantimonites go into solution with great difficulty. For such minerals Chamot recommends the use of a small capsule or crucible covered with a glass slide, together with a suitable holder. In this way the reagent can be brought to boiling point and held there a minute or so. ${ }^{66}$ Pyrargyrite, which is almost insoluble when heated on a slide, was tested in a crucible and was easily broken up chemically. About twice the quantity of material ordinarily used on a glass slide is necessary when using the capsule. The liquid is boiled to dryness. The capsule is allowed to cool, then leached with three or four drops of dilute acid. This is picked up by means of the capillary tube and transferred to a glass slide and there subjected to the usual treatment.

The reagent most frequently used is $1: 1$ nitric acid (1 volume of concentrated $\mathrm{HNO}_{3}$, specific gravity 1.42 , plus 1 volume of $\mathrm{H}_{2} \mathrm{O}$ ).

Chamot, E. M., op. cit., p. 153.

"Idem, p. 293, fig. 153 . 
Aqua regia (3 volumes of concentrated $\mathrm{HCl}$ plus 1 volume of concen. trated $\mathrm{HNO}_{3}$ ) is used where a strong oxidizing action is required. Where it is necessary to make use of a strong oxidizing action with. out introducing chlorine ions, fuming nitric acid is used. The stronger reagents have a tendency to spread over the slide, and it is greatly preferable to use $1: 1 \mathrm{HNO}_{3}$ where possible. The reagent to be used is always determined by trial.

Capillary tubes.-The process of separating a filtrate from a precipitate is described above (p. 118). The capillary tubes used by the writer are made from a tube of soft glass originally 3.6 millimeters in outer diameter, 2.2 millimeters in inner diameter, and about 16 centimeters long. The tube is held, one end in each hand, and heated in the middle over a hot flame of small cross section; when red-hot, but before it begins to sag, it is quickly removed from the flame and elongated by pulling the hands away from each other. (See fig. 13.) During the heating the tube should be given a rotary motion in order to heat the cross section equally. The tube should not be elongated while still in the flame. An ordinary

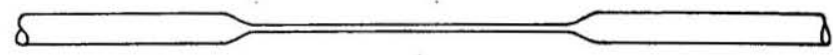

Frgure 13.-Capillary tube. About natural size

Bunsen flame is not suitable for this purpose as it does not concentrate the heat in a sufficiently small cross section, and the improvised alcohol lamp already described does not give a hot enough flame. A blowpipe flame has been found most satisfactory. The writer uses a blowpipe operated by a power blast and fixed in a stand. An ordinary hand blowpipe serves just as well, but the services of an assistant who operates the blowpipe will be necessary, as the worker will need both of his hands to draw out the tubing.

Ordinarily the capillary tube thus obtained is longer than is convenient to work with and is therefore broken off at a point 8 to 10 centimeters from the constriction. The inner diameter of the capil. lary part generally ranges from 0.06 to 0.12 millimeter. If the diameter is smaller than 0.06 millimeter it is difficult to blow the liquid out of the tube. If larger than 0.12 millimeter, the liquid is blown out too easily and tends to form a drop of large diameter in comparison to its volume. With a little practice satisfactory tubes can easily be made. The diameter of the capillary part is remarkably uniform over a comparatively great length, and it is still more remarkable that the walls of the tube rarely coalesce anywhere. Tubes 2 feet or more in length can be prepared in this manner with the exercise of a little skill. The longer the tube is drawn out the smaller the diameter tends to be. Usually the best practice is to draw it out about a foot and then break it off to a convenient length. 


\section{METHODS OF APPLYING TESTS}

The criterion used in the recognition of nearly every element is the character of crystals formed by precipitation within the drop. It is therefore desirable to regulate the conditions of the tests so as to obtain crystals of maximum size and constant character. The reagent used in bringing about the precipitation should have a higher concentration than the unknown elements in the drop to be tested. If the concentration of ions that unite to form the precipitate is too great, the crystals will be small and poorly formed. If the concentration is too low, the precipitate will not form at all. The happy medium at which the crystals will be of maximum size is determined by experiment. For instance, the crystals of silver bichromate are of maximum size and most satisfactory in character when a small fragment of ammonium bichromate is placed in a 0.5 per cent solution of silver nitrate. Similarly the crystals of copper mercuric thiocyanate, which are the most useful for the recognition of copper, are most satisfactory in size and shape when a solution of potassium mercuric thiocyanate is added to a 0.5 per cent solution of copper nitrate. The writer believes that approximately this concentration is most satisfactory for most elements. Efforts have been made, therefore, to start with a quantity of material that will yield approximately this concentration of the metallic element in the drop to be tested. If the concentration is too great the fact will be manifest at once by the abundance of the precipitate and its tendency to be amorphous. The test can therefore be repeated on new material, or the drop can be diluted and a portion picked up by the capillary tube, transferred to a new place on the slide, and tested. On the other hand, efforts have been made to ascertain the minimum amount of mineral necessary to give a definite positive test for the element in question. Chamot ${ }^{67}$ says :

It is essential that reagents be always applied in the best possible manner and in concentrations and under condtions such as will lead to the separation of a solid crystalline phase in a very short period of time. It is therefore necessary that we first ascertain the best method of procedure for each particular reagent. Most failures to obtain satisfactory results when attempting microchemical reactions are due to a lack of appreciation of the importance of this fact. Manuals of microchemical analysis usually neglect to state definitely the best manner of adding a reagent to the drop to be tested, assuming that the investigator will ascertain for himself the conditions which will yield him products most easily identified. Under similar conditions as to concentration, acidity, and manner of reagent application, the crystalline phase will not only almost invariably separate with the same habit, but the crystals will usually develop to the same size and will l'e upon the object slide in each experiment in the same positions with respect to faces.

Chamot, E. M., op. cit., p. 298. 
- Chamot describes the typical procedures used by him and desig. nates them by roman numerals. His nomenclature is followed by the present writer. Some of his procedures had no application in the writer's tests and are not here described.

Method $I$ is the one most used in this investigation and is prefer. able to any other." "A drop of the reagent is placed near a drop of the solution to be tested. By means of a platinum wire or drawn. out glass rod a tiny channel is made to flow from the reagent into the test drop, the slide being tipped to facilitate the flow, but under no condition should the two drops merge completely." If the slide is not perfectly clean, it will be almost impossible to form a channel between the drops, as the liquid will not wet the glass. Except in a test for iron a sharp-pointed needle is better

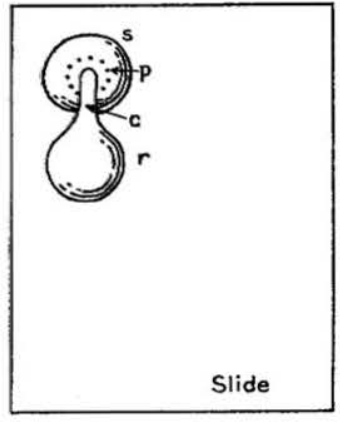

Figure 14.-Di a g r a m showing Chamot's method I. The reagent drop (r) has been made to flow into the drop to be tested (s) through a tiny channel (c). The precipitate separates at p. than a platinum wire or glass rod for joining the drops. A platinum wire of small diameter will bend too easily, and a tiny glass rod is too fragile. The presence of a little iron gained from the needle can be ignored in by far the greater proportion of tests. Where iron is objectionable a sharpened match serves very well to form the channel. The size of the drops used by the writer is smaller than that pictured by Chamot (see fig. 14), the maximum being 2 millimeters and the average about 1.5 millimeters. The distance between the edges of the drops is usually not more than 1 millimeter.

In method III a tiny fragment of the solid reagent is added to a drop of the solution of the substance to be tested. This method is of frequent use. It furnishes a high concentration of ions of the reagent but is open to the objection that the quantity of reagent ap. plied is not so easily controlled as when applied in liquid of a definite concentration. Beginners almost invariably use too large a frag. ment of the reagent, and this causes a large proportion of the reagent to crystallize out in advance of the precipitate desired and to mask it. The writer crushes the solid reagent and spreads it over a dark surface and by means of a moist sharpened match picks up about the smallest fragment that can be seen by the unaided eye and transfers it to the center of the drop. If, after microscopic observation, more reagent seems to be needed it can be supplied.

${ }^{\text {es }}$ Chamot, E. M., op. cit., p. 299. 
In method III-A a drop of the reagent is added to the test drop at its center. ${ }^{69}$ An example of this method is given on page 157 of this paper. A modification not described by Chamot might be termed method III-B. The drop containing the unknown elements is evaporated over the microburner or, if slow evaporation is desirable, by merely exposing it to the atmosphere. The liquid reagent that causes the precipitation is added to the dried residue. An example of this method is the dimethyl glyoxime test for nickel (p. 134). Where it is desirable to keep the drop from spreading, the test drop is slowly evaporated, and when it is nearly dry but still moist a drop of the reagent is added directly to it. An example of this procedure is the ammonium molybdate test for arsenic.

The operations of joining the drops or dropping the fragment in the test drop are best done under the microscope, using a Leitz No. 2 or a Bausch \& Lomb 32-millimeter objective and a I Leitz No. 3 eyepiece. After the crystals of the desired precipitate begin to form, higher powers can be used. The Leitz No. 4 is the objective most used for final observation. It combines high magnification with clearness of vision and good perspective. Objectives of higher magnification than this are only rarely needed.

In both of the textbooks cited more than one test is usually given for one element. The choice between tests is determined only by experiment. Some of the tests are of such great delicacy that they indicate the presence of an element when its proportion in a mineral is so small that it does not appear in the formula given in the usual texts. For instance, when chalcocite is taken into solution in nitric acid, the test drop will usually stain a solution of potassium mercuric thiocyanate pink, indicating the presence of iron in chalcocite. In such tests experience is the best guide in judging whether the element is present in sufficiently large amounts to be recorded in the formula. These tests can also be supplemented by tests of lower delicacy.

The microchemical method can not be used to distinguish between two minerals having the same elements, such as boulangerite and quinkenite, or petzite and sylvanite. Moreover, the susceptibility of this method is strictly limited, and reliable tests can not be expected on quantities of material represented by areas on the section less than 0.17 millimeter in diameter. It is useless to make tests on grains of a mineral so minute that they can be seen only with objectives of stronger magnification than the 16-millimeter lens. With such material the identity of a mineral must be based on other characteristics, such as behavior in polarized light, cleavage, color, hardness, and etching behavior.

Chamot, E. M., op. cit., p. 302. 


\section{SENSITIVITY OF MICROCHEMICAL TESTS}

In the outline preceding the description of each test is given the concentration of the most dilute solution that will yield a satisfac. tory reaction. It is recognized that to assign a definite value for allowable dilution is always more or less arbitrary. The figures given are based largely on the result of the writer's experience in teaching microchemistry to advanced students in economic geology. They are believed to represent the most dilute solutions that ordi. narily will yield consistent results to the average student.

The molality of a solution with respect to an element is defined as the weight of the element in grams per liter of solution divided by the atomic weight of the element. For instance, a solution of lead acetate containing 0.379 gram of salt per 100 cubic centimeters is 0.01 molal with respect to lead; likewise a solution of cobalt nitrate containing 0.291 gram of salt per 100 cubic centimeters is 0.01 molal with respect to cobalt. These two solutions have an equal number of atoms of the metallic element per unit volume.

A comparison of the molalities of the limiting solutions, shown in the following table; gives a truer conception of the sensitivity of the tests than the concentrations of the solutions themselves:

Approximate sensitivities of microchemical tests

\begin{tabular}{|c|c|c|c|c|}
\hline \multirow[b]{2}{*}{ Element } & \multirow[b]{2}{*}{ Test } & \multirow{2}{*}{$\begin{array}{l}\text { Concentration of most dilute solu- } \\
\text { tion (grams of salt per } 100 \text { cubic } \\
\text { centimeters of solution) }\end{array}$} & \multicolumn{2}{|c|}{$\begin{array}{l}\text { Concentration } \\
\text { of element }\end{array}$} \\
\hline & & & $\begin{array}{l}\text { Gram } \\
\text { per } 100 \\
\text { cubic } \\
\text { centi. } \\
\text { meters }\end{array}$ & $\begin{array}{l}\text { Molst. } \\
\text { ity of } \\
\text { solution }\end{array}$ \\
\hline Zinc. & Potassium mercuric thiocyanate..- & $0.01 \mathrm{Zn}\left(\mathrm{C}_{2} \mathrm{H}_{3} \mathrm{O}_{2}\right)_{2} .2 \mathrm{H}_{2} \mathrm{O}$ & 0.00297 & 0.0045 \\
\hline Copper & 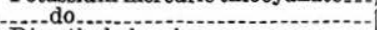 & $0.01 \mathrm{Cu}\left(\mathrm{NO}_{3}\right)_{2.3} \mathrm{H}_{2} \mathrm{O}$ & .00263 & .00013 \\
\hline Nickel. & Dimethyl glyoxime & $0.02 \mathrm{Ni}\left(\mathrm{NO}_{3}\right)_{2} .6 \mathrm{H}_{2} \mathrm{O}$ & .00406 & .0 \\
\hline Do. & Potassium mercuric thiocyanate... & & .00406 & \\
\hline Cobalt. & 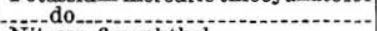 & $0.01 \mathrm{Co}\left(\mathrm{NO}_{3}\right) .6 \mathrm{H}_{2} \mathrm{O}$ & .00203 & .000 ill \\
\hline Do. & Nitroso $\beta$-naphthol & $0.02 \mathrm{CoCl}_{2} .6 \mathrm{H}_{2} \mathrm{O} \ldots$ & .00496 & $.000 \mathrm{ell}$ \\
\hline Do... & Potassium nitrite & $0.05 \mathrm{Co}\left(\mathrm{NO}_{3}\right)_{2} .6 \mathrm{H}_{2} \mathrm{O}$.. & .1010 & .0171 \\
\hline Iron & Potassium mercuric thiocyanate... & $0.02 \mathrm{Fe}\left(\mathrm{NO}_{3}\right)_{3} .9 \mathrm{H}_{2} \mathrm{O} \ldots$ & .00277 & .00000 \\
\hline $\begin{array}{ll}\text { Do... } \\
\text { Do }\end{array}$ & Potassium ferrocyanide & do & .00277 & .0000 \\
\hline Do... & Ammonium hydroxide & $0.03 \mathrm{Fe}\left(\mathrm{NO}_{3}\right)_{3} .9 \mathrm{H}_{2} \mathrm{O} \ldots$ & 00415 & .0074 \\
\hline Mercury...... & $\begin{array}{l}\text { Cupferron } \\
\text { Cobalt mercuric thiocyanate }\end{array}$ & $0.005 \mathrm{HgCl} \mathrm{Cl}_{2} \ldots . .$. & $\begin{array}{l}.00115 \\
.0037\end{array}$ & .0002 \\
\hline Lead........... & Potassium iodide & $0.005 \mathrm{~Pb}\left(\mathrm{C}_{2} \mathrm{H}_{3} \mathrm{O}_{2}\right) \cdot 3 \mathrm{H}_{2} \mathrm{O}$ & .00273 & .00018 \\
\hline Do.. & 1:5 hydrochloric acid. & $0.10 \mathrm{~Pb}\left(\mathrm{C}_{2} \mathrm{H}_{3} \mathrm{O}_{2}\right) \cdot 3 \mathrm{H}_{2} \mathrm{O} \ldots$ & .0546 & .000 \\
\hline Do . & Triple nitrite & $0.01 \mathrm{~Pb}\left(\mathrm{NO}_{3}\right)_{2}$ & .00625 & .0001 \\
\hline Silver.... & Ammonium bichromate..... & $0.02 \mathrm{AgNO}_{3} \ldots$ & .0093 & \\
\hline Do... & 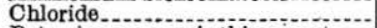 & $\ldots$ do $\ldots$ & .0093 & \\
\hline & Potassium mercuric thiocyanate... & do.................... & .0093 & \\
\hline Gold & Pyridine and hydrobromic acid .... & $0.02 \mathrm{HAuCl}_{4}$ & .0116 & \\
\hline Do ... & Stannous chloride & $0.10 \mathrm{HAuCl}_{1}$ & .058 & \\
\hline Arsenic ... & Ammonium molybdate........... & $0.01\left(\mathrm{NH}_{4}\right)_{3} \mathrm{AsO} .3 \mathrm{H}_{2} \mathrm{O} \ldots \ldots$ & .00304 & .0 \\
\hline Do & Potassium iodide & $0.02\left(\mathrm{NH}_{4}\right)_{3} \mathrm{AsO} .3 \mathrm{H}_{2} \mathrm{O}$ & .00608 & \\
\hline Antimony.... & Cesium double chloride... & $0.02 \mathrm{SbCl}_{3-.}$ & .01056 & \\
\hline ismuth. & Cesium double iodide & $0.01 \mathrm{SbCl}_{3}$ & .00528 & 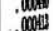 \\
\hline $\begin{array}{l}\text { ismuth...... } \\
\text { Do........ }\end{array}$ & Cesium double chloride.......... & $0.02 \mathrm{Bi}\left(\mathrm{NO}_{3}\right)_{3} .5 \mathrm{H}_{2} \mathrm{O} \ldots \ldots$ & .0086 & \\
\hline in & 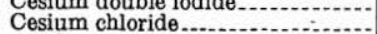 & $\begin{array}{l}0.01 \mathrm{Bi}(\mathrm{NO} \\
0.01 \mathrm{SnCl}\end{array}$ & .00626 & .00 \\
\hline ellurium & - & $0.01 \mathrm{Te}$ (in $\mathrm{HCl}$ ). & .01 & 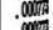 \\
\hline 0 & Potassium iodide & & & $(m$ \\
\hline & stannous chioride. & & & \\
\hline
\end{tabular}




\section{REAGENTS}

Below is a list of the reagents used.

\section{Acids and ammonia}

1:1 $\mathrm{HNO}_{5}-1$ volume of concentrated $\mathrm{HNO}_{3}$ (specific gravity 1.42) added to 1 volume of $\mathrm{H}_{2} \mathrm{O}$.

1:7 $\mathrm{HNO}_{3}-1$ volume of $1: 1 \mathrm{HNO}_{3}$ added to 3 volumes of $\mathrm{H}_{2} \mathrm{O}$.

1 per cent $\mathrm{HNO}_{3}-1$ volume of concentrated $\mathrm{HNO}_{3}$ added to 99 volumes of $\mathrm{H}_{2} \mathrm{O}$. Aqua regia-1 volume of concentrated $\mathrm{HNO}_{3}$ added to 3 volumes of concentrated

$\mathrm{HCl}$ (specific gravity 1.09).

1:5 $\mathrm{HCl}-1$ volume of $1: 1 \mathrm{HCl}$ added to 2 volumes of $\mathrm{H}_{2} \mathrm{O}$.

5 per cent $\mathrm{HCl}-5$ volumes of $1: 1 \mathrm{HCl}$ added to 45 volumes of $\mathrm{H}_{2} \mathrm{O}$.

$\mathrm{NH}_{6} \mathrm{OH}-$ concentrated solution.

1 per cent $\mathrm{NH}_{4} \mathrm{OH}-1$ volume of concentrated $\mathrm{NH}_{4} \mathrm{OH}$ added to 99 volumes of $\mathrm{H}_{2} \mathrm{O}$.

1 per cent acetic acid-1 volume of glacial $\mathrm{H}\left(\mathrm{C}_{2} \mathrm{H}_{3} \mathrm{O}_{2}\right)$ added to 99 volumes of $\mathrm{H}_{2} \mathrm{O}$.

Pyridine-hydrobromic acid-1 volume of pyridine added to 9 volumes of 40 per cent $\mathrm{HBr}$.

\section{Salt solutions}

Dimethyl glyoxime-1 volume of 2 per cent dimethyl glyoxime in alcohol, added to 1 volume of $\mathrm{H}_{2} \mathrm{O}$.

Potassium mercuric thiocyanate- $\mathbf{5}$ per cent solution in water. (See directions for preparing salt, p. 128.)

a-nitroso $\beta$-naphthol-Dissolve 1 gram of nitroso $\beta$-naphthol in 33 cubic centimeters of cold glacial acetic acid, add equal volume of $\mathrm{H}_{2} \mathrm{O}$, and filter.

Cupferron- 6 per cent solution in $\mathrm{H}_{2} \mathrm{O}$.

Acetate reagent-Dissolve 0.5 gram of sodium acetate and 0.5 gram of copper acetate in 100 cubic centimeters of 1 per cent acetic acid.

Stannous chloride-2 per cent solution in 1:5 HCl.

Ammonium molybdate-1.5 per cent solution in 1:7 $\mathrm{HNO}_{3}$.

\section{Solids}

Cesium chloride- $\mathrm{CsCl}$.

Potassium iodide-KI.

Potassium nitrite- $\mathrm{KNO}_{2}$.

Potassium ferrocyanide- $\mathrm{K}_{4} \mathrm{Fe}(\mathrm{CN})_{0}$.

Cobalt nitrate- $\mathrm{Co}\left(\mathrm{NO}_{3}\right)_{2} \cdot 6 \mathrm{H}_{2} \mathrm{O}$.

Potassium thiocyanate-KCNS.

Ammonium bichromate- $\left(\mathrm{NH}_{4}\right)_{2} \mathrm{Cr}_{2} \mathrm{O}_{7}$.

Rubidium chloride- $\mathrm{RbCl}$.

Copper acetate- $\mathrm{Cu}\left(\mathrm{C}_{2} \mathrm{H}_{3} \mathrm{O}_{2}\right)_{2}$.

Sodium acetate- $\mathrm{NaC}_{2} \mathrm{H}_{3} \mathrm{O}_{2}$.

Sodium bismuthate- $\mathrm{NaBiO}_{3}$.

Calcium acetate- $\mathrm{Ca}\left(\mathrm{C}_{2} \mathrm{H}_{3} \mathrm{O}_{2}\right)_{2}$.

Potassium mercuric thiocyanate is not purchasable in the market but can be made up in the laboratory as follows:

Prepare about 50 cubic centimeters of a 10 per cent solution of potassium thiocyanate (KCNS). Also prepare a solution of mer- 
curic chloride $\left(\mathrm{HgCl}_{2}\right)$ by dissolving 10 grams of the salt in about 160 cubic centimeters of water. Slowly titrate the KCNS solution into a beaker containing all of the $\mathrm{HgCl}_{2}$. No precipitate will appear until about 13 cubic centimeters of the KCNS solution has been added. At that point precipitation of white mercuric thio. cyanate proceeds rapidly, according to the reaction $2 \mathrm{KCNS}+$ $\mathrm{HgCl}_{2}=\mathrm{Hg}(\mathrm{CNS})_{2}+2 \mathrm{KCl}$. This reaction is reversible, and only part of the mercury can be precipitated in this manner. Add 2 cubic centimeters more of KCNS solution and let stand 5 minutes. If more KCNS is added at this stage the mercuric thiocyanate already formed will begin to dissolve, which is to be avoided.

Filter the mercuric thiocyanate precipitate, after washing it in order to get rid of adhering traces of $\mathrm{KCNS}$ and $\mathrm{KCl}$, and transfer

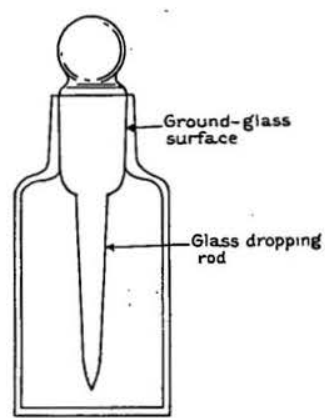

Figure 15.-Glass-stoppered dropping bottle. About natural size it into a 250 cubic centimeter beaker. The mer. curic thiocyanate precipitate is soluble in in excess of KCNS with the formation of the double salt $\mathrm{K}_{2} \mathrm{Hg}(\mathrm{CNS})_{4}$. Titrate 10 per cent KCNS solution into the beaker containing the mercuric thiocyanate precipitate. The solution should be added slowly, and the contents of the beaker stirred constantly. The first chemical change is the formation of $\mathrm{KCNS} . \mathrm{Hg}(\mathrm{CNS})_{\text {: }}$, which is almost insoluble in water but is soluble in an excess of KCNS with the formation of $2 \mathrm{KCNS} . \mathrm{Hg}(\mathrm{CNS})_{2}$. When about 23 cubic cen. timeters of KCNS solution has been added, the precipitate dissolves suddenly. It is essential to

be careful at this stage to keep the excess of KCNS at a minimum. The resulting solution, if of the proper concentration, is ready for use. It is best, however, to evaporate the solution to dryness, weigh the salt, and dissolve it in about twenty times its weight of water. About 5.5 grams of solid $2 \mathrm{KCNS} . \mathrm{Hg}(\mathrm{CNS})_{2}$ is formed by the procedure above set forth.

The solution should be tested by merging a drop of it into a drop of 1 per cent cobalt nitrate solution, weakly acidified with $\mathrm{HNO}_{3}$. A blue precipitate should form immediately and should consist of isolated or interlocking needles. (See pl. 4, A.) If precipitation is delayed and branching forms appear at the edges of the drop (see pl. $5, A$ ), an excess of KCNS in the reagent is indicated. In this event it is probably quicker and more satisfactory to start from the beginning again than to attempt to purify the reagent by recrystal. lizing it from water. The entire procedure, with the exception of the final evaporation, does not require more than 30 minutes. The reagent should also be tested by merging it with a drop of 0.01 per cent cobalt nitrate solution. When the drop approaches dryness ting 
blue cobalt mercuric thiocyanate prisms will appear, provided the reggent is of satisfactory purity. Copper, iron, and zinc do not require the same degree of purity of reagent as cobalt.

The preceding list of reagents comprises 10 acids and other liquids leaving no residue on evaporation, 7 salt solutions, and 12 solids. The liquid reagents are best kept in 15 cubic centimeter glassstoppered dropping bottles. (See fig. 15.) Cáre must be taken, however, to remove the glass dropping rods from the bottles from time to time, as the rods tend to become "frozen" to the necks of: the bottles owing to evaporation of the salts. ${ }^{70}$ The solids are kept in cylindrical phials with cork stoppers.

\section{DETAILS OF TESTS}

\section{ZINC}

\section{Potassium mercuric thiocyanate test.-}

Reagent: $5 \%$ potassium mercuric thiocyanate solution in $\mathrm{H}_{2} \mathrm{O}$.

Product: Zinc mercuric thiocyanate $\left(\mathrm{Zn}(\mathrm{CNS})_{2} \cdot \mathrm{Hg}(\mathrm{CNS})_{2}\right)$, white feathery crosses.

Limit: $0.01 \%$ zinc nitrate solution. ${ }^{n}$

This is the only test used by the writer for the determination of inc. Another test, the sodium carbonate reaction described by Chamot, is characteristic when obtained, but the writer has never had much success with it, as the conditions under which the zinc carbonato tetrahedra form are not always obtainable. The potassium mercuric thiocyanate test, on the other hand, is reliable, delicate, and easily controlled.

Potassium mercuric thiocyanate $\left(\mathrm{K}_{2} \mathrm{Hg}(\mathrm{CNS})_{4}\right.$; see p. 128) is the most useful reagent for microchemistry yet discovered. It yields delicate and satisfactory tests with solutions containing zinc, copper. cobalt, nickel, iron, silver, and gold, provided that only one metal is present in the solutions. With lead the reaction is less satisfactory. The reactions are given below for comparison:

Zinc nitrate: White feathery crosses.

Copper nitrate: Yellowish-green mosslike clumps and rounded prisms.

Cobalt nitrate: Indigo-blue prisms.

Nickel nitrate: Brown spherulites.

Iron nitrate: No precipitate, but solution is colored red.

Silver nitrate: White curdy amorphous precipitate.

Gold chloride: White amorphous precipitate; more gelatinous than that of silver.

Lead nitrate: Colorless prisms; not satisfactory.

\footnotetext{
"Glass-stoppered dropping bottles similar to that shown in the sketch may be obtained trom Arthur H. Thomas Co., Philadelphia, Pa., for approximately 40 cents each.

"This outline was devised by Putnam, Roberts, and Selchow. See articles already cited.
} 
The acetates of these metals work equally well when acidified with $\mathrm{HNO}_{3}$.

All reactions involving the use of potassium mercuric thiocyanate succeed best in the presence of $\mathrm{HNO}_{3}$, although they work fairly well with neutral solutions. Potassium mercuric thiocyanate does not precipitate zinc copper, etc., from alkaline solutions.

Tests were made to determine the delicacy of the zinc reaction by successively diluting a solution of zinc acetate in 1 per cent $\mathrm{HNO}_{3}$. The solution was free from cadmium.

1\%: The solution gave a strong reaction but was too concentrated, Amorphous grains and distorted forms predominate. ${ }^{72}$

$0.5 \%$ : Highly satisfactory. Feathery crosses predominate.

$0.1 \%$ : Highly satisfactory. The simpler forms predominate.

$0.05 \%$ : Satisfactory. Simple crosses, some with arrowhead points common. $0.01 \%$ : Test distinct but requires time. A few small simple crosses are observed. This is probably the limit of allowable dilution.

The most common forms are illustrated in Plate 2.

The presence of other metals in addition to zinc in the unknown solution modifies the results above given.

Iron does not materially alter the shape of the zinc mercuric thiocyanate crystals but stains them a light lavender to purplish red, the color depending on the concentration of iron. This color is best observed in reflected light. Most specimens of sphalerite when tested in this way show the presence of more or less iron.

Copper has a far-reaching effect on the zinc reaction, the two elements tending to form isomorphous crystals. If one element is in excess of the other, part of the predominating element will unite with all of the subordinate element to form mixed crystals, and the remainder of the predominating element will form pure crystals. Acetates of zinc and copper acidified with $\mathrm{HNO}_{3}$ were mixed and tested with $\mathrm{K}_{2} \mathrm{Hg}(\mathrm{CNS})_{4}$, with the following results:

$0.5 \%$ zinc acetate ${ }^{73}$ and $0.005 \%$ copper acetate.-Pure zinc crystals alone form. No sign of copper.

$0.5 \%$ zinc acetate and $0.01 \%$ copper acetate.-Almost no evidence of copper. Some of the zinc crosses show a faint violet tinge in reflected light.

$0.5 \%$ zinc acetate and $0.2 \%$ copper acetate.-Same as normal zinc test, but crystals are tinged violet in reflected light; almost opaque in transmitted light. In addition to crosses, simpler crystals also seen. (See fig. 16, a.)

$0.5 \%$ zinc acetate and $0.5 \%$ copper acetate.-Same as preceding, but color is a light violet. Simpler crosses predominate over feathered crosses. (See fig. 16, b.)

\footnotetext{
${ }^{72}$ In this paper solutions are usually expressed, for convenience, as percentages of the dissolved salt. This is slightly inaccurate, because 1 cubic centimeter of the solution weighs more than 1 gram. The concentrations as given are actually grams of salt per 100 cubic centimeters of solution.

7s This solution is actually 1 cubic centimeter of 1 per cent zinc acetate added to 1 cubic centimeter of 0.01 per cent copper acetate. The percentages as given above are percentages of each salt expressed in terms of total solution.
} 
$0.5 \%$ zinc acetate and $0.1 \%$ copper acetate.-Isolated purple prisms like those shown in Figure 16, o. Crystals fairly transparent. Crosses like preceding subordinate.

$0.5 \%$ zinc acetate and $0.25 \%$ copper acetate.-Isolated prisms like preceding most characteristic. Color violet to purple; some opaque. No crosses observed.

$0.5 \%$ zinc acetate and $0.5 \%$ copper acetate.-Clumps of prisms which have the same form as the copper compound but are dark purple. In addition some normal yellowish-green copper prisms are present. (See fig. 16, d.)

$0.25 \%$ zinc acetate and $0.5 \%$ copper acetate.-Typical copper crystals greatly predominate, but in addition there are clumps of sporelike prisms, opaque in transmitted light and
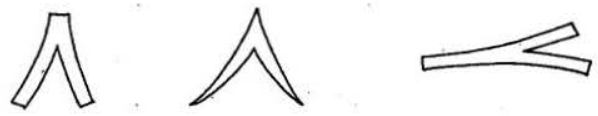
dark purple in reflected light.

$0.1 \%$ zinc acetate and $\mathbf{0 . 5 \%}$ copper acetate.-Same as above, but clumps of mixed crystals are smaller and less numerous. (See
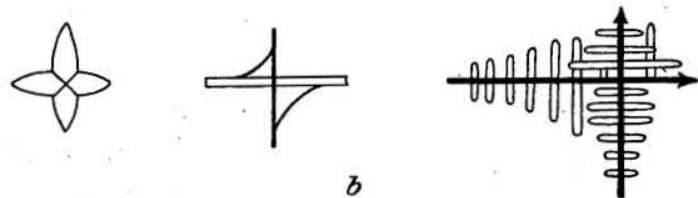

fig. 16, e.)

$0.05 \%$ zinc acetate and $0.5 \%$ copper acetate.-Similar to preceding, but mixed crystals still less numerous.

$0.02 \%$ zinc acetate and $\mathbf{0 . 5 \%}$ copper acetate.-No-indication of zinc.

The conclusion is that a very small percentage of copper in a fairly concentrated zinc solution is readily detected by this method; the pres-
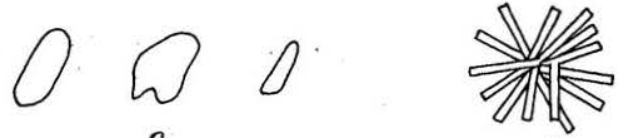

d ence of a small percentage of zinc in a fairly concentrated copper solution is also easily detected, although the sensitivity of the test is less than when the zinc predominates.

Cobalt and zinc also form mixed crystals. Mixtures of cobalt and inc nitrates yielded the following results:

$0.5 \%$ zinc nitrate and $0.5 \%$ cobalt nitrate.-Almost no indication of the presence of zinc. Some deep-blue crosses and V-shaped crystals have the forms of the normal zinc compound. Normal deep-blue cobalt prisms predominate. $0.5 \%$ zinc nitrate and $0.1 \%$ cobalt nitrate.-Zinc crosses predominate. Most of them are normal, but some are tinged with blue.

$0.5 \%$ zinc nitrate and $0.05 \%$ cobalt nitrate.-Zinc crosses, most of them normal but a few tinged light blue. 
$0.5 \%$ zinc nitrate and $0.02 \%$ cobalt nitrate.-Normal zinc crosses, a few of which are tinged pale blue in reflected light. About the limit of possible dilution for cobalt.

The conclusion is that a small percentage of cobalt can be detected in a concentrated zinc solution but zinc can not be detected in a strong cobalt solution. The cobalt should be removed by means of potassium nitrite (p. 138) before testing for zinc with potassium mercuric thiocyanate.

\section{According to Chamot, ${ }^{74}$}

Inorganic salts of cadmium yield $\mathrm{Cd}(\mathrm{CNS})_{2} \cdot \mathrm{Hg}(\mathrm{CNS})_{2}$ in brilliant colorless, probably orthorhombic prisms, usually several times as long as broad, but the uppearance of these prisms varies with the conditions which obtain at the time of their formation, as, for example, the concentration, depth of the test drop, amount of reagent added, acidity, etc. These variations are, however, not of a kind to render the test doubtful, long prisms either singly or in groups being the rule.

Even a small amount of cadmium destroys the feathery and branched character of the skeletons of zinc-mercury thiocyanate, owing to the formation of mixed crystals, and there generally result crystallites of the shape of an arrowhead. Small amounts of zinc in the presence of much cadmium will usually escape detection.

\section{COPPER}

\section{Potassium mercuric thiocyanate test.-}

Reagent: $5 \% \mathrm{~K}_{2} \mathrm{Hg}(\mathrm{CNS})_{4}$ in $\mathrm{H}_{2} \mathrm{O}$.

Product: Copper mercuric thiocyanate in greenish-yellow mosslike aggregates and prisms.

Limit: 0.01 copper nitrate solution.

The test for copper is carried out precisely like the corresponding test for zinc. Clumps of mosslike or fernlike dendrites are precipitated in solutions of relatively high concentration. The color of the aggregate is yellow with a tinge of green. Where the dilution is greater, aggregates of acicular prisms form. These give parallel extinction in polarized light. They are generally light greenish yellow, but some of the thicker crystals may be distinctly olive-green. (See pl. 3.)

Extraneous elements, for the most part at least, do not seem to interfere with the copper test. Copper, however, may interfere with tests for other elements. It is frequently desirable to separate the copper from some other elements that are soluble in nitric acid. This can be done by taking the copper into solution by means of a drop of ammonia, transferring the drop to a different position on the slide, evaporating off the ammonia, and taking the copper into solution again by means of nitric acid. The copper is then proved by means of potassium mercuric thiocyanate, as already described. If the concentration of copper is sufficiently great in the ammoniacal

" Chamot, E. M., op. cit., p. 355. 
solution the familiar blue color is readily recognized, and the final test is not absolutely necessary. It should be remembered that nickel, cobalt, zinc, silver, arsenic, and some other elements are also soluble in ammonia. None of these seem to interfere with the copper test.

The potassium mercuric thiocyanate test for copper is so satisfactory and "foolproof" that none other is needed. The only fault that can be found with it is that some minerals which are not supposed to contain copper yield good tests for it. For instance, a good copper test can usually be obtained from bismuthinite. The student should be on guard for results of this kind and ignore faint tests.

Potassium iodide.-If a fragment of potassium iodide is placed in a $1: 5 \mathrm{HCl}$ solution containing copper, a finely divided light-yellow amorphous precipitate of cuprous iodide $(\mathrm{CuI})$ is formed. This reaction is sensitive and useful, in that it often appears in tests for other elements, especially for antimony, bismuth, and tin. Copper should be eliminated before testing for these elements. When the copper iodide precipitate appears, it is best to wash the original residue with successive drops of $1: 7 \mathrm{HNO}_{3}$ and test each one for copper with $\mathrm{K}_{2} \mathrm{Hg}(\mathrm{CNS})_{4}$ solution until a negative reaction is obtained. Then proceed with the test for $\mathrm{Sb}, \mathrm{Bi}$, or $\mathrm{Sn}$.

Cesium chloride.-When a fragment of cesium chloride is placed in a $1: 5 . \mathrm{HCl}$ solution cortaining copper ions, orange or golden colored needles form at the edge of the drop. This result, like that with potassium iodide, sometimes appears in tests for other elements and does not interfere to any extent with the tests for $\mathrm{Sb}, \mathrm{Bi}$, or $\mathrm{Sn}$.

When successive fragments of $\mathrm{KI}$ and $\mathrm{CsCl}$ are placed in a 1:5 $\mathrm{HCl}$ solution containing copper, irregular, more or less rounded grains form. These are opaque in transmitted light and white in obliquely reflected light. In addition. long colorless prisms with ragged sides sometimes form. It is best to remove all copper before making the $\mathrm{KI}$ and $\mathrm{CsCl}$ test for antimony or bismuth.

Triple nitrite reaction.-The triple nitrite reaction is described on page 145. If the test is being made for copper, lead is supplied, preferably in the form of a solution containing 0.5 per cent of lead scetate and 0.5 per cent of sodium acetate in 1 per cent acetic acid.

\section{Dimethyl glyoxime test.-}

\section{NICKEL}

Reagent: $2 \%$ dimethyl glyoxime solution in alcohol plus an equal volume of $\mathrm{H}_{2} \mathrm{O}$. Product: Nickel dimethyl glyoxime $\left(\mathrm{Ni}\left(\mathrm{C}_{4} \mathrm{H}_{7} \mathrm{~N}_{2} \mathrm{O}_{2}\right)_{2}\right)$.

Limit: $0.02 \%$ nickel nitrate.

The familiar dimethyl glyoxime test used in qualitative analysis is also the best for microchemical tests. This reagent is not soluble in $15122-31-10$ 
water but is readily soluble in alcohol and other organic solvents. Alcohol spreads over the slide and consequently should be diluted with water. Accordingly a 2 per cent solution of the reagent in alcohol is diluted by adding an equal volume of water. The resultant mixture is a 1 per cent solution of dimethyl glyoxime. The solution continually precipitates white crystalline dimethyl glyoxime, which sinks and tends to form a cake. For this reason it is best to avoid shaking or stirring the solution. The dimethyl glyoxime crystals are easily recognized under the microscope and do not obscure the nickel test.

The precipitation takes place in neutral, weakly alkaline, and weak acetic acid solutions. No precipitation occurs in $\mathrm{HNO}_{3}$ or $\mathrm{HCl}$ solutions. Cobalt retards the formation of the precipitate, and therefore if much cobalt is present it should be removed by starting with an acetic acid solution, precipitating the cobalt with potassium nitrite, and testing the filtrate with dimethyl glyoxime. The familiar pink precipitate is usually amorphous at first, but a felty mass of tiny pink needles soon forms. When observed with a high power these needles are seen to be strongly pleochroic between colorless and pink. With the analyzer inserted, the interference color is usually light blue.

In testing an unknown mineral for nickel the material is dissolved in 1:1 $\mathrm{HNO}_{3}$ or aqua regia. The residue is leached with 1: $7 \mathrm{HNO}_{3}$, and the filtrate is transferred to a new place on the slide and evaporated over the microflame. A drop of 1 per cent $\mathrm{NH}_{4} \mathrm{OH}$ is added to the residue from the evaporation in order to precipitate any iron present. If iron were left in solution it would likewise be thrown down by dimethyl glyoxime as a reddish precipitate. Hence it is advisable to eliminate the iron by precipitation with ammonia before making the nickel test. The ferric hydroxide formed occludes a considerable percentage of nickel, but most of the nickel remains in solution. The ammonia is allowed to evaporate in the air. When nearly dry the dimethyl glyoxime solution is added directly to the nearly evaporated ammoniacal solution. The gelatinous ferric hydroxide does not interfere with the formation or observation of the nickel precipitate. If the concentration of nickel ions is very low, the test drop can be evaporated and the reagent, made slightly ammoniacal, is added directly to the residue. Dimethyl glyoxime gave a good test on a 0.02 per cent solution but a poor test on a 0.01 per eent solution of nickel nitrate.

Potassium mercuric thiocyanate test.-

Reagent: $5 \% \mathrm{~K}_{2} \mathrm{Hg}$ (CNS) $)_{4}$ in $\mathrm{H}_{2} \mathrm{O}$.

Product: Nickel mercuric thiocyanate ( $\left.\mathrm{NiHg}(\mathrm{CNS})_{4}\right)$.

Limit: $0.02 \% \mathrm{Ni}\left(\mathrm{NO}_{3}\right)_{2}$ solution. 
Potassium mercuric thiocyanate added to a $1: 7 \mathrm{HNO}_{3}$ solution containing nickel ions precipitates round spherulites that are light brown in transmitted light and white in reflected light. Sometimes an amorphous white powder is first precipitated at the edge of the drop, but it tends to redissolve with the formation of the spherulites. The nickel mercuric thiocyanate is more soluble than the corresponding cobalt precipitate and does not form till the drop is partly evaporated. -The spherulites are tinged pink if iron is present in colution. (See pl. 4, D.) This test is exceedingly useful in that the nickel spherulites often appear when testing for cobalt and iron.

\section{COBALT}

\section{Potassium mercuric thiocyanate test.-}

Reagent: $5 \% \mathrm{~K}_{2} \mathrm{Hg}(\mathrm{CNS})_{4}$ in $\mathrm{H}_{2} \mathrm{O}$.

Product: Cobalt mercuric thiocyanate $\left(\mathrm{CoHg}(\mathrm{CNS})_{4}\right)$, in indigo-blue prisms. Limit: $0.01 \%$ cobalt nitrate solution.

Tests for cobalt are carried out exactly like those for copper and inc. The precipitate separates as dark-blue prisms, usually in clusters. (See pl. $4, A$.) With dilute solutions (0.05 per cent or less of $\left.\mathrm{Co}\left(\mathrm{NO}_{3}\right)_{2}\right)$, blue spherulites also form. The formation of spherulites has not been sufficiently emphasized by Chamot and is not mentioned by Behrens and Kley. If much nickel is present the cobalt mercuric thiocyanate will not form as prisms but will appear at the edge of the drop as blue spherulites. (See pl. 4, C.) If the ratio of cobalt to nickel is relatively high the spherulites will show radiating fibers, each giving parallel extinction. With still higher ratios incipient prisms form at the surface of the spherulite, and finally a mass of radiating quills something like a prickly pear results. The presence of other elements also causes the formation of the spherulites. Potassium cobalt nitrite when taken into solution with nitric acid and tested with potassium mercuric thiocyanate will cause the formation of the spherulites.

Although the potassium mercuric thiocyanate test for cobalt is as delicate as the corresponding test for copper, the cobalt compound tends to supersaturate and frequently does not begin to be precipitated until evaporation of the drop is well advanced. This is particularly true when spherulites predominate over prisms. Once formed, however, the precipitate is not readily taken into solution by diluting the drop with water or nitric acid.

The spherulites may appear anywhere within the drop but are most commonly found at the circumference. At first thought it rould seem that the absence of the more typical prisms and the formation of blue spherulites in their place is a serious drawback to this test. However, one soon becomes familiar with their appear- 
ance and accepts them as proof of the presence of cobalt. Experi. ments performed on mixtures of cobalt and nickel chlorides indicate that if the ratio of nickel to cobalt is greater than $1: 5$, only spheru. lites will form. If the ratio is less than that, some well-formed crystals will be produced in addition to the spherulites.

A comparatively strong nitric acid solution $\left(1: 7 \mathrm{HNO}_{3}\right)$ gives the best results, although the test will work in a neutral, weak nitric or acetic acid solution. It does not work well in hydrochloric or sulphuric acid. With weak nitric acid the precipitate tends to as. sume branching and mosslike forms rather than prisms.

Experiments were made to determine the sensitiveness of the cobalt test by successively diluting a solution of cobalt nitrate.

1.0\%: Excellent test.

$0.5 \%$ : Even better.

$0.1 \%$ : Good test but requires a minute or more. The crystals appear at the edge of the drop. Most of the precipitate appears as small formless rounded grains. The blue eclor is sufficiently diagnostic.

$0.05 \%$ : Similar to preceding.

$0.01 \%$ : Blue spherulites first appear at the edge of the drop. After a while tiny needles also appear, both singly and in clusters. This is probably close to the limit of possible dilution.

The formation of the triple thiocyanate of cobalt, zinc, and mercury is described on page 131. Copper and cobalt do not combine to form mixed crystals under the same conditions, but each forms its own precipitate. Copper comes down first in characteristic yellowish-green crystals, and cobalt later in blue prisms and spherulites.

Iron does not interfere with the formation of typical cobalt prisms unless in relatively concentrated solution. Ferric ions stain the thio. cyanate solution reddish but do not form an insoluble compound. Mixtures of ferric and cobalt nitrates in 1:7 $\mathrm{HNO}_{3}$ in various proportions were tested as follows:

$0.5 \% \mathrm{Co}\left(\mathrm{NO}_{3}\right)_{2}$ and $0.05 \% \mathrm{Fe}\left(\mathrm{NO}_{3}\right)_{3}$.-Normal cobalt prisms.

$0.5 \% \mathrm{Co}\left(\mathrm{NO}_{3}\right)_{2}$ and $0.10 \% \mathrm{Fe}\left(\mathrm{NO}_{3}\right)_{3}$. - Normal cobalt prisms predominate; also some formless grains much darker but distinctly blue in strong transrerse illumination.

$0.5 \% \mathrm{Co}\left(\mathrm{NO}_{3}\right)_{2}$ and $0.25 \% \mathrm{Fe}\left(\mathrm{NO}_{3}\right)_{3}$.-Cobalt prisms; some normal, but most of them very dark blue. The blue color appears with strong illumination.

$0.5 \% \mathrm{Co}\left(\mathrm{NO}_{3}\right)_{2}$ and $0.5 \% \mathrm{Fe}\left(\mathrm{NO}_{3}\right)_{3}$.-Same as preceding, but solution turns very pink, and prisms are envelope-shaped.

$0.25 \% \mathrm{Co}\left(\mathrm{NO}_{3}\right)_{2}$ and $0.5 \% \mathrm{Fe}\left(\mathrm{NO}_{3}\right)_{3}$.- Same as preceding. Most of the prisus are opaque, but a few normal cobalt prisms are also seen.

$0.1 \% \mathrm{Co}\left(\mathrm{NO}_{3}\right)_{2}$ and $0.5 \% \mathrm{Fe}\left(\mathrm{NO}_{3}\right)_{3}$.-Cobalt prisms black but show blue at thin edges.

$0.05 \% \mathrm{Co}\left(\mathrm{NO}_{3}\right)_{2}$ and $0.5 \% \mathrm{Fe}\left(\mathrm{NO}_{3}\right)_{3}$.- Solution strong red. Prisms light purplish to black. No sign of blue.

$0.02 \% \mathrm{Co}\left(\mathrm{NO}_{3}\right)_{2}$ and $0.5 \% \mathrm{Fe}\left(\mathrm{NO}_{3}\right)_{3}$.- Solution red to purple. Small seedlike grains purplish to black.

$0.05 \% \mathrm{Co}\left(\mathrm{NO}_{3}\right)_{2}$ and $2.5 \% \mathrm{Fe}\left(\mathrm{NO}_{3}\right)_{3}$.-Very good test. Prisms purple to black. 
T. \$. GEOLOGICAL SURVEY
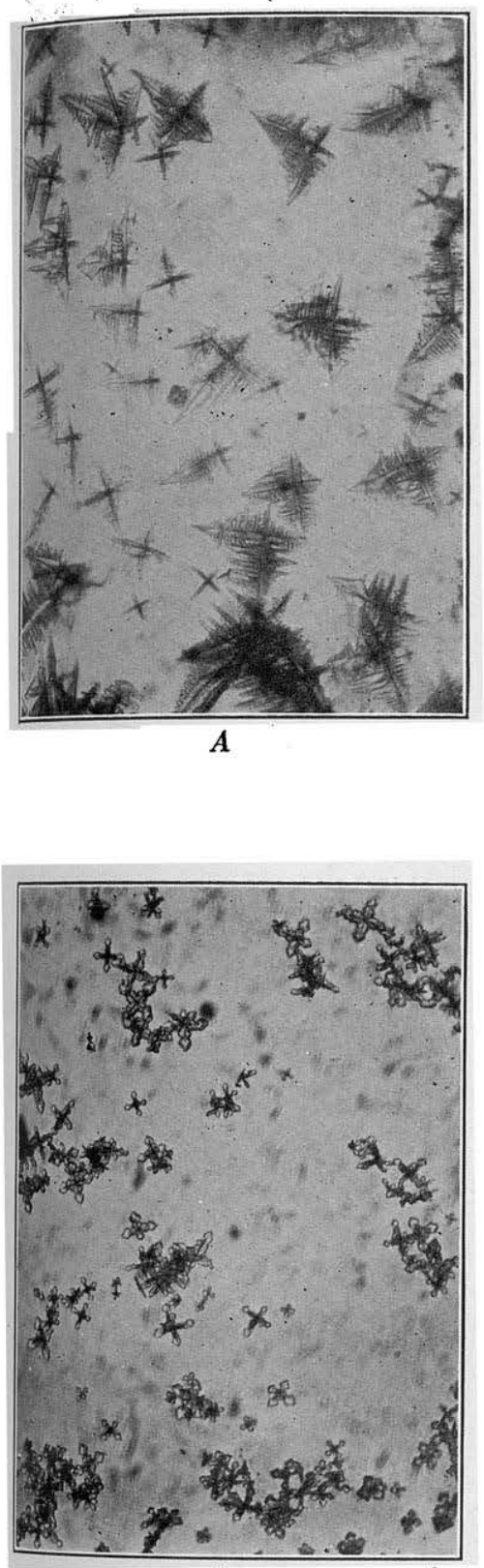

C
BULLETIN 825 PLATE 2
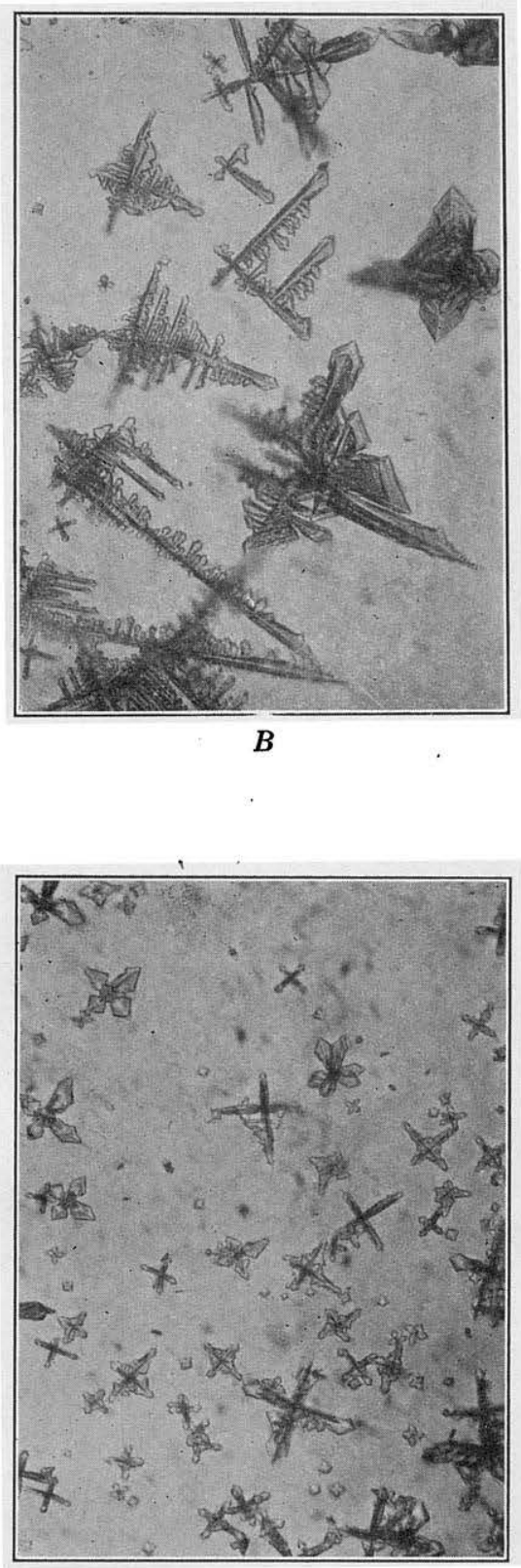

D

MICROCHEMICAL TESTS FOR ZINC

A, B Tinc mercuric thiocyanate crystals obtained from a 0.5 per cent zinc nitrate solution, $\times 128$; $C, D$, zinc mercuric thiocyanate crystals obtained from a 0.1 per cent zinc nitrate solution, $X 208$; (4) . . . . 

U. S. GEOLOGICAL SURVEY
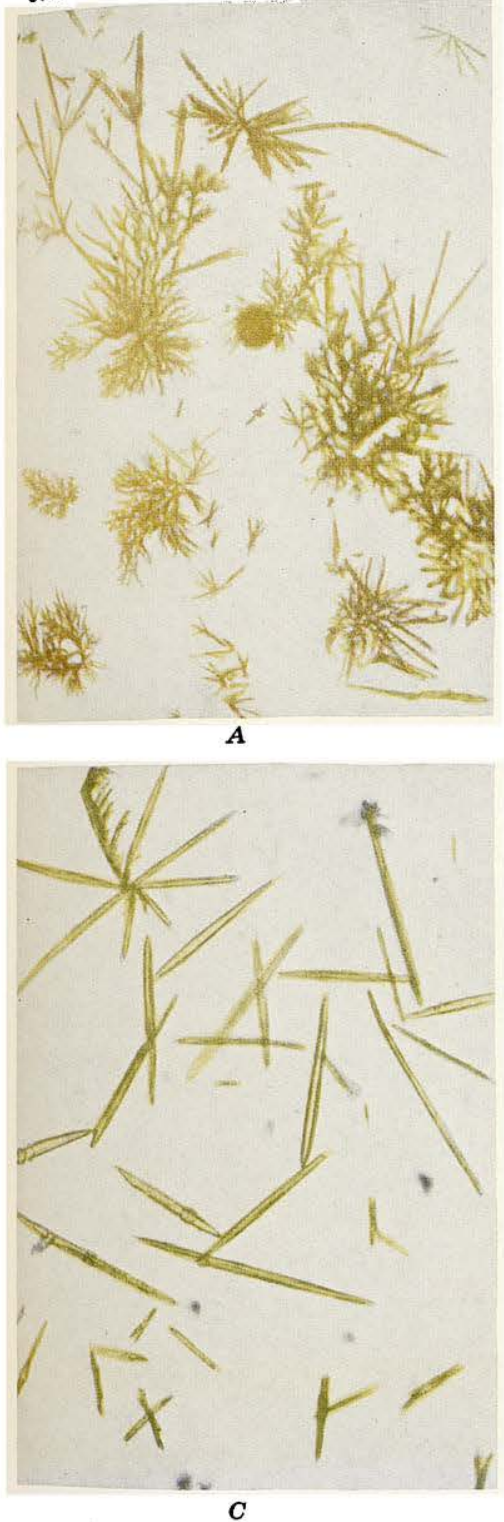

BULLETIN 825

PLATE 3

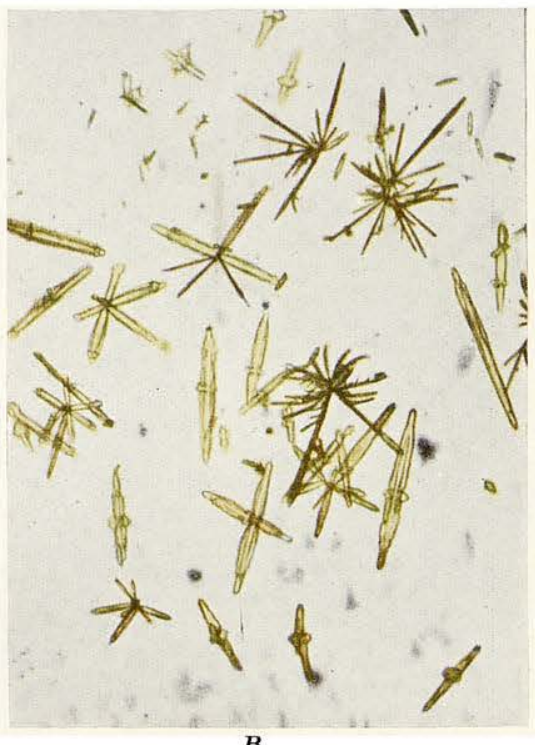

$B$

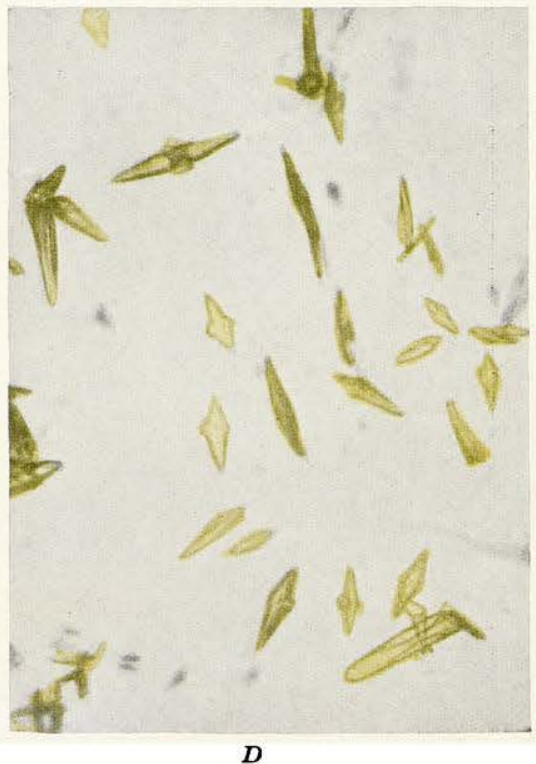

MICROCHEMICAL TESTS FOR COPPER

$A$, Copper mercuric thiocyanate crystals. Obtained from a $0.5 \%$ copper nitrate solution. $X 130$. $B$, Copper mercuric thiocyanate crystals. Obtained from a $0.1 \%$ copper nitrate solution. $\times 130$. $C, D$, From same solution as $B$. 


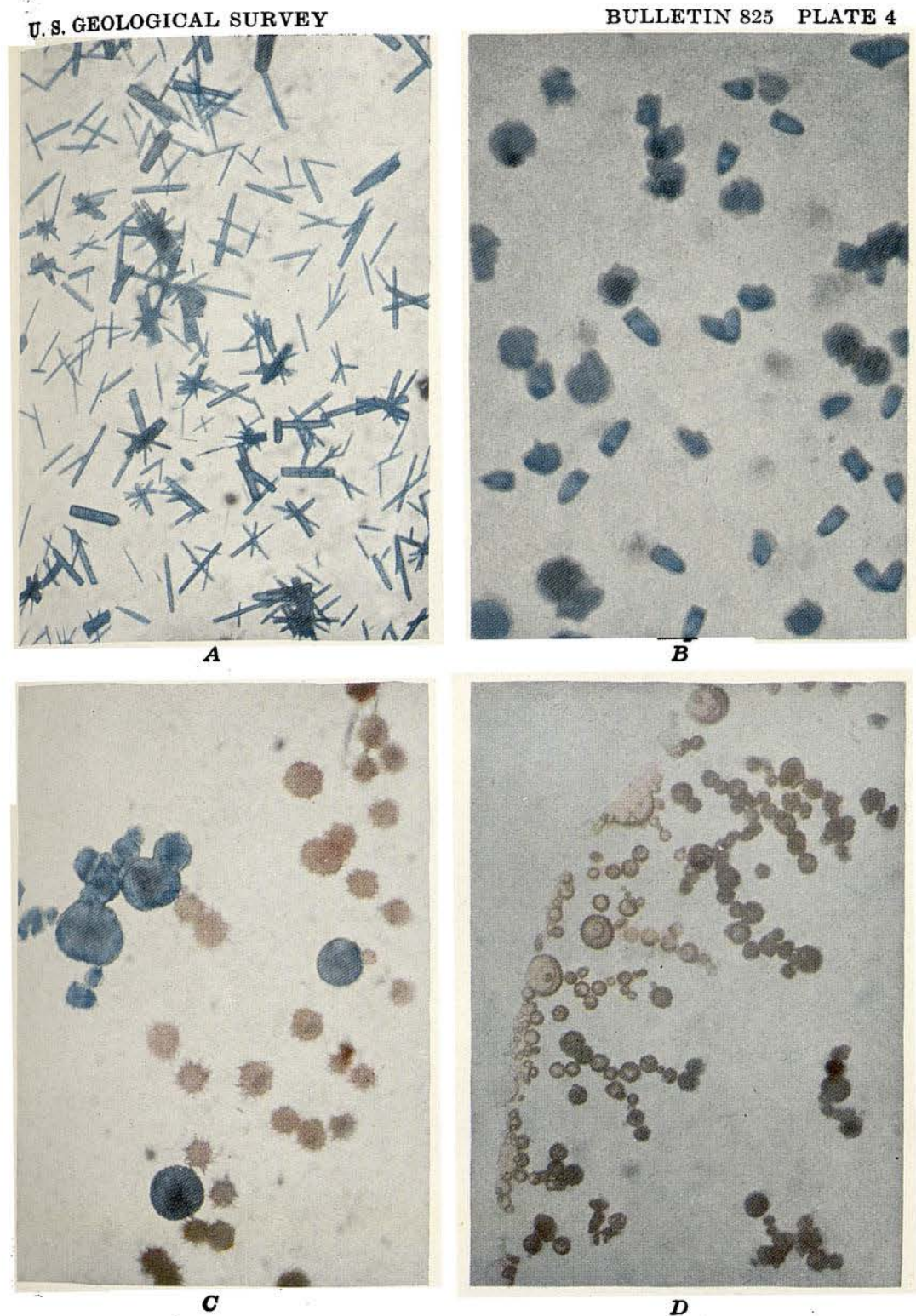

$D$

MICROCHEMICAL TESTS FOR COBALT AND NICKEL

$A$, Cobalt mercuric thiocyanate prisms. From a $1 \%$ cobalt nitrate solution. $\times 130$. B, Cobalt mercuric thiocyanate prisms and spherulites. From a solution containing $0.5 \%$ cobalt nitrate and $0.1 \%$ nickel nitrate. $\times 260 . C$, Cobalt and nickel mercuric thiocyanate spherulites. From a solution containing $0.5 \%$ cobalt nitrate and $0.5 \%$ nickel nitrate. $\times 210$. $D$, Nickel mercuric thiocyanate spherulites. From a solution containing $0.5 \%$ nickel nitrate. $\times 210$. 

O. \$. GEOLOGICAL SURVEY.

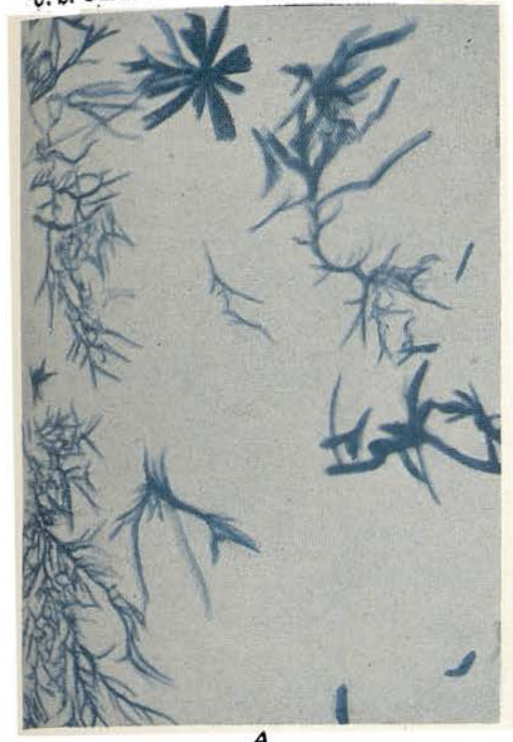

$A$

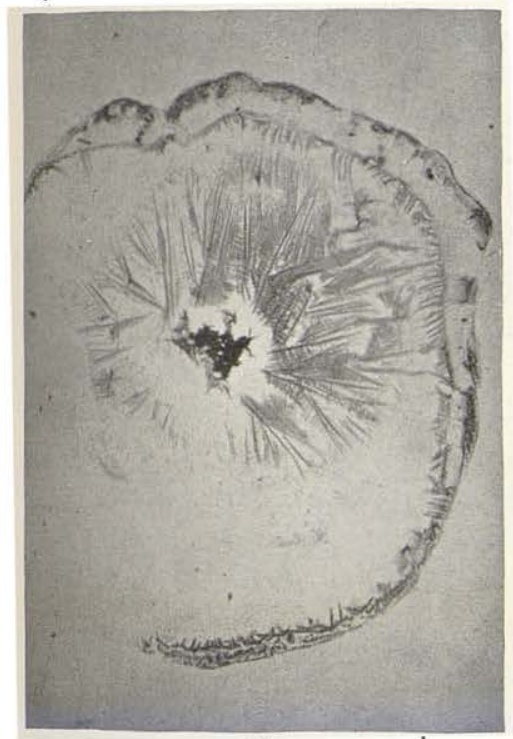

C
BULLETIN 825

PLATE 5

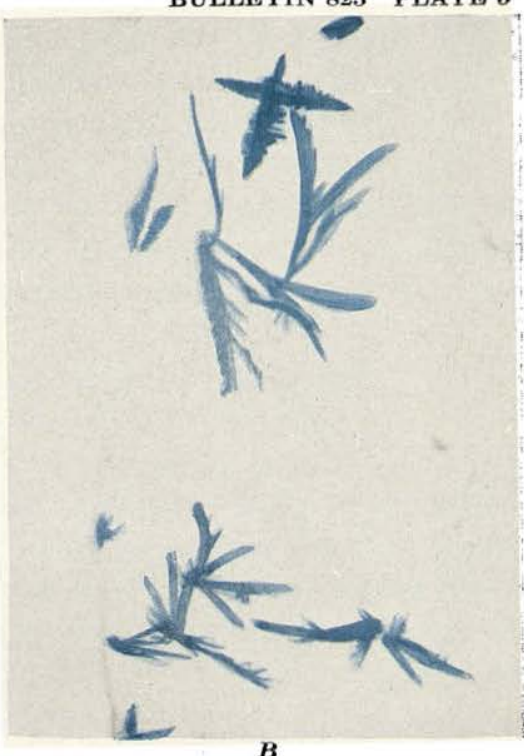

$\boldsymbol{B}$

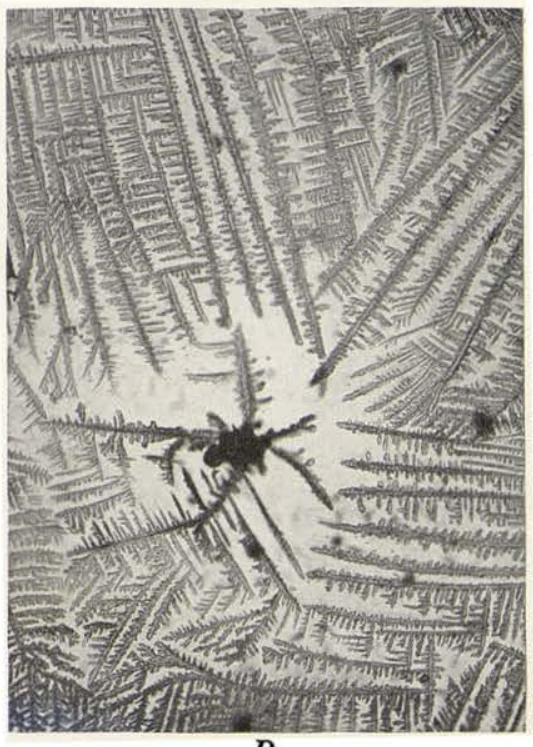

\section{$\boldsymbol{D}$}

$A$, $B$, Cobalt mercuric thiocyanate. From a $0.5 \%$ mercuric chloride solution. $\times 210$. C, Mercuric chloride residue. Obtained by evaporating cinnabar (HgS) powder to dryness with aqua regia. $\times 23$. $D$, Mercuric chloride residue. Obtained by evaporating cinnabar powder to dryness on a glass slide with aqua regia. $\times 130$. 

$0.05 \% \mathrm{Co}\left(\mathrm{NO}_{3}\right)_{2}$ and $5.0 \% \mathrm{Fe}\left(\mathrm{NO}_{3}\right)_{3 .}-\mathrm{Black}$ prisms and spherulites. Some of the thinnest prisms are purplish.

Summarizing the above experiments shows that a moderate concentration of iron does not interfere with the thiocyanate test for cobalt, but a high concentration modifies the shape and color of the precipitate. As iron occurs in most cobalt minerals, especially the arsenides, the student should familiarize himself with the appearance of the mixed crystals. A potassium mercuric thiocyanate test yielding a red solution and purple to black prisms calls for a confirmatory test for cobalt by the nitroso $\beta$-naphthol or potassium nitrite method.

Nitroso $\beta$-naphthol test.-

Reagent: $1.5 \%$ solution of $\alpha$-nitroso $\beta$-naphthol in $50 \%$ acetic acid.

Product: $\left(\mathrm{C}_{10} \mathrm{H}_{6}(\mathrm{NO}) \mathrm{O}\right)_{2} \mathrm{Co}$, a bulky amorphous red precipitate.

Limit: $0.02 \% \mathrm{CoCl}_{2}$.

Nitroso $\beta$-naphthol is an organic compound used in quantitative analysis for the gravimetric determination of cobalt in the presence of nickel. The precipitate is voluminous and comes down quickly. For this reason this method has some advantages over the potassium nitrite method next described. McKinstry ${ }^{75}$ recommends nitroso $\beta$-naphthol as a microchemical test for cobalt.

The reagent is made by dissolving 1 gram of nitroso $\beta$-naphthol in 33 cubic centimeters of, cold glacial acetic acid, adding an equal volume of water, and filtering. The addition of water greatly increases the surface tension of the liquid and prevents the drop from spreading over the slide.

The cobalt precipitate is almost identical in appearance and character with metallic selenium. Its color ranges from crimson in relatively large quantities to brick-red in lesser amounts. The test works best in strong $\mathrm{HCl}$ solutions, as the corresponding nickel compound is soluble in $\mathrm{HCl}$. However, when method I is used, the precipitate does not agglutinate but spreads over the entire area covered by the drop. As the precipitate is not collected into definite crystals, the color of the precipitate in very small masses is uncertain, and the test thus loses much of its sensitiveness.

A better method is to evaporate the unknown solution quickly over the microflame. The material tends to collect as a circular ridge around the periphery of the drop. The slide is cooled, and a drop of reagent is applied directly to the dried residue. A crimson to brickred ring much bulkier than the original residue forms in its place. Nickel does not interfere with this test. The corresponding nickel compound is light lemon-yellow and is comparatively soluble in acetic acid. As the drop evaporates, the nickel compound begins to come down but does not obscure the red cobalt compound already formed.

\footnotetext{
${ }^{i 5}$ McKinstry, H. E., Microchemical methods in the identification of opaque minerals: Econ. Geology, vol. 22, p. 830, 1927.
} 
A solution containing 1 per cent of $\mathrm{NiCl}_{2}$ and 0.02 per cent of $\mathrm{CoCl}_{2}$ gave an excellent test for cobalt by this method.

By far the greatest objection to this test is the fact that iron is likewise completely precipitated with the cobalt. The iron precipitate is chocolate-brown in color and if present in any quantity com. pletely obscures the cobalt precipitate. Hence iron must be removed from solution before testing for cobalt.

Copper and chromium are likewise precipitated from acid solutions, but aluminum, lead, cadmium, manganese, nickel, mercury, and zinc are not precipitated by nitroso $\beta$-naphthol. ${ }^{76}$

Iron can not be separated from cobalt by making the solution ammoniacal, as the ferric hydroxide has a strong tendency to occlude any cobalt present. ${ }^{77}$ This was confirmed by the writer. A drop of solution containing 0.5 per cent of $\mathrm{CoCl}_{2}$ and 0.5 per cent of $\mathrm{FeCl}_{3}$ was evaporated over a microflame. After cooling, concentrated $\mathrm{NH}_{4} \mathrm{OH}$ was added to the residue. The filtrate was transferred to another place on the slide and evaporated to dryness. The residue was taken into solution in 1: $7 \mathrm{HNO}_{3}$ and tested for cobalt by means of potassium mercuric thiocyanate. Only a trace of cobalt was found.

A more successful attempt was made by means of cupferron. (See p. 141.) A solution containing $\mathrm{CoCl}_{2}$ and $\mathrm{FeCl}_{3}$ was evaporated to dryness. The residue was cooled and then leached with a 6 per cent solution of cupferron, which was likewise evaporated to dryness. The residue was leached with $1: 7 \mathrm{HNO}_{3}$, and the filtrate was transferred to another place on the slide and evaporated to dryness. The residue was cooled and then leached with nitroso $\beta$-naphthol reagent. The red cobalt ring formed immediately, and no trace of the brown iron compound was observed. Mixtures of various proportions of $\mathrm{CoCl}_{2}$ and $\mathrm{FeCl}_{3}$ were tested in this manner. A solution containing 0.5 per cent of $\mathrm{FeCl}_{3}$ and 0.05 per cent of $\mathrm{CoCl}_{2}$ gave a good test for cobalt. One containing 0.5 per cent of $\mathrm{FeCl}_{3}$ and 0.03 per cent of $\mathrm{CoCl}_{2}$ gave a distinct cobalt precipitate, but the amount.was so small that the writer could not be sure of its color, which is the only criterion in judging the test.

Potassium nitrite test.-

Reagent: Solid potassium nitrite $\left(\mathrm{KNO}_{2}\right)$.

Produet: Potassium cobalt nitrite $\left(3 \mathrm{KNO}_{2 .} \mathrm{Co}\left(\mathrm{NO}_{2}\right)_{2.11 / 2} \mathrm{H}_{2} \mathrm{O}\right)$ in small yellow isometric cubes.

Limit: $0.5 \% \mathrm{Co}\left(\mathrm{NO}_{3}\right)_{2}$.

The potassium nitrite test for cobalt is described in detail both by Chamot and by Behrens and Kley. The solution containing the

\footnotetext{
* Mellor, J. W.. A treatise on chemical analysis, p. 394, London, 1913.

" Clennell, J. E., The estimation of cobalt by the nitroso $\beta$-naphthol method: Mining Mag. (London), vol. 36, p. 272, 1927.
} 
cobalt, generally a nitric acid solution, is evaporated to dryness. It is then redissolved in a drop of 1 per cent acetic acid, a fragment of potassium nitrite is added, and the drop is gently heated but not sufficiently to evaporate it. Potassium cobalt nitrite separates out in tiny yellow cubes, so minute as to simulate an amorphous or finely granular deposit. With high magnification the grains seem almost spherical and greatly resemble those of ammonium arsenomolybdate, described on page 156.

Behrens and Kley ${ }^{78}$ state that this reaction is especially valuable for separating cobalt from other metals, because in the absence of dements of the barium group only cobalt is precipitated. Chamot is also very favorably disposed toward this reaction. He says ${ }^{79}$ : "This test has its greatest value in a negative way, since failure to obtain the very insoluble double nitrite may be considered as indicative of the absence of cobalt."

The test would be very useful indeed if it were as reliable as these comments would indicate. The present writer can not agree with their conclusions. The results of many days of experimentation with cobalt solutions of various strengths indicate that the test is unreliable when the concentration of cobalt is less than 0.5 per cent of cobalt nitrate. There is evidently a strong tendency for the double nitrite to supersaturate, and it may not come down at all. The test requires a great deal of manipulative skill. The fragment of $\mathrm{KNO}_{2}$ must not be too large, and the solution must not be heated too much, or it will evaporate before the precipitate has a chance to come down. For these reasons this test is not recommended as a test for cobalt, but it is occasionally useful in removing an excess of cobalt before testing for other elements.

\section{IRON}

\section{Potassium mercuric thiocyanate test.-}

Reagent: $5 \% \mathrm{~K}_{2} \mathrm{Hg}(\mathrm{CNS})_{4}$ in $\mathrm{H}_{2} \mathrm{O}$.

Product: No precipitate forms, but solution turns pink.

Limit: $0.02 \% \mathrm{Fe}\left(\mathrm{NO}_{3}\right)_{3}$.

Any thiocyanate solution will give a delicate test for iron. The iron must be in the ferric state in this as in the other tests described in this paper. As a matter of fact, the iron is certain to be left in the ferric state if the mineral to be determined is taken into solution or broken down by means of nitric acid or aqua regia.

The residue is then leached with 1: $7 \mathrm{HNO}_{3}$, and the drop is transferred to another place on the slide and joined to a drop of the reagent by method I (p. 124). Ferric nitrate solutions of 0.2 per cent

Behrens, H., and Kley, P. D. C., op. cit., p. 77.

"Chamot, E. M., op. cit., p. 413. 
or greater concentration immediately turn pink near the junction of the two solutions. If the concentration is less than 0.2 per cent the color may not appear until the test drop is nearly dry. The pink to purple color then appears at the periphery of the drop.

This test is very valuable in that it often appears when testing for other elements. Many minerals containing 1 per cent or more of iron will often give a distinct thiocyanate test for iron when, as \& matter of fact, iron is not expressed in the formulas usually given for these minerals. The writer has obtained distinct tests for iron with sphalerite and chalcocite, for instance. This is not an objection to the test but merely records the fact that more or less iron is found in many ore minerals that are not usually classed as iron-bearing.

Potassium ferrocyanide test.-

Reagent: Solid potassium ferrocyanide $\left(\mathrm{K}_{4} \mathrm{Fe}(\mathrm{CN})_{6}\right)$. Product: Amorphous blue precipitate (prussian blue). Limit: $0.02 \%$ ferric nitrate or chloride solution.

A fragment of potassium ferrocyanide when added to a solution containing ferric ions will give the familiar prussian-blue precipitate. It is amorphous but sufficiently characteristic in color to be diagnostic. When transverse illumination is used the color is likely to be greenish. The blue color is best observed by tilting the mirror and observing the precipitate in oblique illumination.

The greatest objection to this test is that many elements are precipitated by potassium ferrocyanide, and if any of them are present with the iron the blue color of the iron precipitate may be obscured. In the presence of a predominance of nickel or cobalt, for instance, some other test for iron should be used.

The ferrocyanide test works in neutral or acid solutions but is much more sensitive in the presence of acid. The writer could not get a blue precipitate with neutral ferric nitrate solutions in a concentration of less than 0.1 per cent, but by adding $\mathrm{HNO}_{3}$ the solution could be diluted to 0.02 per cent and still give a satisfactory test.

The test works equally well in 1:7 $\mathrm{HNO}_{3}$ and 1:5 HCl solutions. The acid should not be more concentrated than this, as the reagent itself contains iron in the molecule, and a strong acid would tend to liberate it and thus contaminate the test.

\section{Ammonium hydroxide test.-}

Reagent: $\mathrm{NH}_{4} \mathrm{OH}$ solution.

Product: Gelatinous ferric hydroxide.

Limit: About $0.03 \%$ ferric nitrate or chloride.

When ammonia is added to a neutral ferric salt or added in excess to an acid ferric solution, the familiar yellow to orange-colored gelatinous ferric hydroxide precipitate immediately forms. In order: to avoid making the drop too large it is best to evaporate off nearly 
all the excess of acid and then add the ammonia directly to the still moist residue. (See method III-B, p. 125.)

The precipitate tends to cling to the glass. It can be washed by three or four successive drops of water, taken again into solution with 1:7 $\mathrm{HNO}_{3}$, and the drop tested for iron with a fragment of potassium ferrocyanide. In this manner iron can be separated from copper, nickel, and many other elements soluble in ammonia. Ordinarily, however, the ferric hydroxide precipitate is so distinctive in appearance that no confirmatory test is needed.

\section{Cupferron test.-}

Reagent: $6 \%$ cupferron solution in $\mathrm{H}_{2} \mathrm{O}$.

Product: Amorphous orange-colored to brick-red precipitate.

Limit: $0.03 \%$ ferric chloride or nitrate.

Cupferron is a name used for convenience to describe the ammonium salt of nitrosophenyl hydroxylamine $\left(\mathrm{C}_{6} \mathrm{H}_{5} \mathrm{~N} . \mathrm{NO} . \mathrm{ONH}_{4}\right){ }^{80}$ It precipitates quantitatively ferric iron, cupric copper, vanadium, zirconium, thorium, cerium, titanium, and tetravalent uranium from strongly acid solutions. The reagent is soluble in water and ammonia but insoluble in acids unless the acids are greatly in excess. For microchemical work cupferron can be considered practically insoluble in 1: $7 \mathrm{HNO}_{3}$ and 1:5 HCl. ${ }^{81}$ In quantitative analysis the cupferron solution is slowly added to the beaker containing iron in a strong hydrochloric acid solution. In this manner the cupferron is kept in solution while the iron is precipitated. But acid should not be added to a cupferron solution, because a white precipitate is immediately thrown down, and it is difficult to get this precipitate into solution again. This property can be utilized in removing iron from acid solutions. The unknown solution is evaporated to dryness, and the cupferron solution added to the residue. The iron, copper, etc., are precipitated. The drop of cupferron is then likewise evaporated. By leaching the precipitate and cupferron residue with $\mathrm{HNO}_{8}$ or $\mathrm{HCl}$, nickel, cobalt, and other elements not precipitated by cupferron are taken into solution, whereas only a small proportion of cupferron is dissolved. The filtrate is transferred and tested for cobalt and other elements. (See p. 138.)

The iron precipitate is orange-colored to brick-red, the color depending on the quantity present. It greatly resembles ferric hydroxide and likewise tends to cling to the glass slide. The copper precipitate is white to gray and crystallizes in orthorhombic needles. The vanadium, titanium, and uranium precipitates are light yellow.

Cupferron solutions slowly decompose and give off a peculiar sweetish odor of nitrosobenzine. The reagent should be made up fresh about once a month.

"Treadwell, F. P., and Hall, W. T., Analytical chemistry, p. 838, New York, 1916.

"Cupferron may be obtained from the Eastman Kodak Co. for 6 cents a gram. 
Summary.-None of the four tests for iron described above preci. pitate the element in definite crystals. However, all the tests are sufficiently distinctive to identify the element. The choice of a test depends chiefly on the other elements present. In acid solutions containing unknown elements the test usually made first is with potassium mercuric thiocyanate, and hence this constitutes the most valuable test for iron. The potassium ferrocyanide test is the most distinctive of the four and can be made in the absence of other elements precipitated by the reagent. The ammonium hydroxide. test is of value in separating iron from copper or nickel. The ferric hydroxide formed, if insufficient in quantity to be distinctive in appearance, can be redissolved in $\mathrm{HNO}_{3}$ or $\mathrm{HCl}$ and tested for iron with $\mathrm{K}_{t} \mathrm{Fe}(\mathrm{CN})_{6}$.

The cupferron test is not as good as the others described, but cupferron serves as a convenient agent to remove iron from acid solutions.

\section{MERCURY}

\section{Double thiocyanate test.-}

Reagents: Solid cobalt nitrate $\left(\mathrm{Co}\left(\mathrm{NO}_{3}\right)_{2} \cdot 6 \mathrm{H}_{2} \mathrm{O}\right)$ and solid potassium thio. cyanate (KCNS).

Product: Potassium mercuric thiocyanate in blue prisms and branching forms. Limit: $0.005 \mathrm{HgCl}_{2}$ in $1 \% \mathrm{HNO}_{3}$.

The test for mercury is the formation of cobalt mercuric thiocyanate-in other words, this test is the reverse of the cobalt test above described. Mercury compounds are distinctive in that they are almost insoluble in $\mathrm{HNO}_{3}$ and $\mathrm{HCl}$ but are readily soluble in aqua regia. The residue $\left(\mathrm{HgCl}_{2}\right)$ when examined under the microscope is seen to be decidedly crystalline. It shows dendrites with rightangle branches, which often form a well-defined grating resembling that of lead nitrate. (See pl. $5, C, D$.)

The mineral is broken down with aqua regia, the residue leached with 1 per cent $\mathrm{HNO}_{3}$, and the solution transferred. A small fragment of cobalt nitrate is added; when this is dissolved, a fragment of potassium thiocyanate is added. Blue prisms and dendrites prove the presence of mercury. (See pl. $5, A, B$.) If the solution is very weak with respect to mercury the formation of the blue crystals is delayed. In very dilute solutions-less than 0.02 per cent $\mathrm{HgCl}_{2}$, for instance-blue spherulites will predominate over well-defined erystals. Sometimes the usually insoluble cobalt mercuric thiocyanate precipitate will not form at all, but when the solution is nearly dry, it will turn a deep-blue color and will precipitate soluble light-blue feathery crystals of unknown composition. If a drop of water is added to this residue, the dark-blue insoluble cobalt mereurie thiocyanate will usually form. 
This test is very sensitive, and a 0.005 per cent mercuric chloride solution will give a satisfactory reaction. The solution must be slightly acidified with nitric acid before adding the solid reagents. Ordinarily the precipitate will not come down at once, as the solution tends to be supersaturated.

\section{IEAD}

\section{Lead iodide test.-}

Reagent: Solid KI.

Product: $\mathrm{PbI}_{2}$; hexagonal lemon-yellow pleochroic plates and disks. Limit: $0.005 \%$ lead acetate.

This is the most useful test for lead. The mineral is taken into solution with nitric acid. Chlorine ions should not be introduced into the solution. The acid is carefully evaporated off with a minimum quantity of heat, otherwise a relatively insoluble basic nitrate or even the oxide will form. It is preferable not to use strong acid, as even with 1:1 $\mathrm{HNO}_{3}$ part of the sulphur is oxidized and the lead precipitated as the insoluble sulphate. Repeated evaporations with weak acid have the same effect. The residue frequently shows a boxwork structure which is one of the indications of the presence of lead. (See pl. 6, D.) Mercury compounds leave a similar residue when broken down by means of aqua regia. Practically all the lead minerals are easily attacked by nitric acid, whereas mercury minerals are almost completely inert to it.

The residue is leached with 0.5 per cent acetic acid, and the drop is transferred and tested with a tiny fragment of KI, using method III. A lemon-yellow precipitate of normal lead iodide is thrown down. ${ }^{82}$ If the concentration of lead ions is relatively large $(0.1$ per cent $\mathrm{Pb}\left(\mathrm{NO}_{3}\right)_{2}$ or greater) well-formed hexagonal plates will usually appear. (See pl. 6, $A, B$.) They may, however, be starshaped or irregular. If the solution is very dilute the crystals will be disk-shaped. Chamot recommends that a weak nitric acid solution be used. It is the writer's experience that better-formed crystals are precipitated from a neutral solution. The plates are greenish yellow in transverse illumination. They apparently show no double refraction with the analyzer inserted but are strongly pleochroic when turned up on edge, and the effect greatly resembles that of biotite plates. The best test, however, is to view them in oblique light and a Leitz No. 3 or equivalent objective. The plates are highly reflecting and glitter like the tinsel on a Christmas tree.

As lead iodide is very soluble in potassium iodide solution, the size of the KI fragment must be kept at a minimum. (See p. 124.)

"This compound is referred to in this bulletin as " normal" lead iodide to distinguish it from the basic iodide and double iodides of lead with potassium, bismuth, and other elements. 
As the drop dries, colorless needless of the double iodide $2 \mathrm{KI} . \mathrm{PbI}_{2}$ usually appear at the edge of the drop. The double iodide needles usually form a boxwork pattern, which is very characteristic and diagnostic of lead. The investigator will soon become familiar with this pattern and will not assume that it represents another element: in addition to lead.

The double iodide of potassium and lead usually forms as a product of the destruction of the normal lead iodide. As the drop evaporates, the destruction of the yellow lead iodide plates begins at the periphery, and the colorless double iodide needles take the place of the plates. As evaporation continues, the wave of destruction advances toward the center until all the normal lead iodide plates have been destroyed and a mat of the double iodide needles has taken its place. Thus the normal iodide is a transitory product, and the observer should have his eye to the microscope when he introduces the KI fragment into the drop; otherwise the normal lead iodide may disappear before being observed. Once destroyed the normal lead iodide can not be reprecipitated from a solution of the residue. However, if the concentration of lead with respect to $\mathrm{KI}$ is high, the normal yellow iodide plates may persist even after the drop has completely evaporated.

Copper and bismuth interfere with this test, and double iodides are formed. Copper can be removed by ammonia, as described on page 132, and the residue afterward tested for lead. In the presence of bismuth it is best to prove the lead by the chloride test described below. Chamot ${ }^{83}$ says that the normal lead iodide can be recrystallized from the double iodide of lead and bismuth by boiling the mixed product with hot water. The writer has never had much success with this procedure. The drop is so small that trying to heat it over a flame will usually result in the entire disappearance of the liquid. Adding water already heated is equally futile, as the drop cools too rapidly when taken from the flame to do much good. The same remarks apply to recrystallizing the normal iodide from hot water.

\section{Chloride test.-}

Reagent: 1:5 HCl.

Product: $\mathrm{PbCl}_{2}$, white orthorhombic needles.

Limit: $0.1 \%$ lead acetate.

If hydrochloric acid is added to a solution containing lead or a soluble salt of lead, characteristic long white acicular crystallites of lead chloride separate out. According to Chamot, ${ }^{84}$ there are also seen feathery dendritic X's and long irregular ragged prisms, but the prism is the only form that has been observed by the writer.

${ }^{8 s}$ Chamot, E. M., op. cit., p. 370, par. 2.

s Idem, p. 372. 
The investigator should familiarize himself with these forms, as they are likely to appear when he is testing for other elements involving the use of $\mathrm{HCl}$, notably antimony and bismuth. It is usually advisable to make an additional test on the lead chloride. This can be done by converting the chloride into the more characteristic normal iodide.

Although generally regarded as insoluble in water, lead chloride is far more soluble than the iodide. Hence if the dried chloride residue is leached with water and the drop transferred, lead iodide in the nsual hexagonal plates can be precipitated by adding a fragment of KI to the drop. This procedure is particularly valuable if bismuth ispresent, because the bismuth will hydrolyze, forming insoluble $\mathrm{BiOCl}$, when water is added to a $\mathrm{BiCl}_{3}$ residue. This is the only method by which the writer has been able to obtain a lead solution tree from bismuth if any bismuth is present in the unknown mineral. Triple nitrite reaction.-

Reagents: $0.5 \%$ of copper acetate and $0.5 \%$ of sodium acetate in $1 \%$ acetic acid; solid potassium nitrite.

Product: Potassium-copper-lead nitrite $\left(2 \mathrm{KNO}_{2} \cdot \mathrm{Cu}\left(\mathrm{NO}_{2}\right)_{2} \cdot \mathrm{PbNO}_{2}\right)_{2}$ in brown to black cubes.

Limit: $0.01 \% \mathrm{~Pb}\left(\mathrm{NO}_{2}\right)_{2}$.

The triple nitrite of potassium, lead, and copper is far more insoluble than the simple nitrites of these elements or the double nitrites formed by any two of them. The triple nitrite occurs as brown to black cubes, also square and rectangular plates. (See pl. 6, c.) The platy crystals are more likely to be brown, and the cubes are usually black. In appearance this compound is striking and highly characteristic. Either lead or copper can be tested for by supplying ions of the other element. However, the potassium mercuric thiocyanate test for copper is so nearly perfect that the triple nitrite reaction is not necessary.

As described by Chamot the solution containing the lead which is to be tested for is evaporated to dryness over the microflame in order to remove strong acids, which otherwise would interfere with the test. The residue is taken into solution with very dilute acetic rid, and successive fragments of sodium acetate, copper acetate, and potassium nitrite are introduced into the solution. If lead is present in sufficient concentration to give the test, a cloud of brown crystals will form and spread out toward the periphery of the drop. When examined in a fairly high magnification (usually a Leitz $N_{0} .4$ or equivalent objective) the crystals are seen to be predominantly cubes.

The sensitivity of the test can be greatly increased by adding a fragment of cesium chloride to the drop. The corresponding triple aitrite of cesium, lead, and copper is identical in appearance with 
the potassium salt and more insoluble. On the other hand, the size of the crystals produced by the cesium salt is correspondingly diminished.

The objection to adding three and possibly four successive frag. ments to a solution is that the quantity of material can not be easily controlled, and the solution tends to be clogged up before the triple nitrite crystals have a chance to form. The writer modifies this procedure by dissolving definite quantities of sodium acetate and copper acetate into the dilute acetic acid. Hence these elements are all supplied in one operation. By experiment it was found that 0.5 per cent of sodium acetate and 0.5 per cent of copper acetate dissolved in 1 per cent acetic acid gave a reagent that worked satisfactorily on test solutions of lead nitrite from 0.01 to 1.0 per cent concentration and on all the lead ore minerals tested.

The lead nitrate solution is evaporated to dryness, and a drop of the acetate reagent is applied to the residue. Then a fragment of potassium nitrite is introduced into the solution. If the concentration of lead is low, the triple nitrite precipitate may not form until the drop is nearly dry. If none forms, a drop of 1 per cent acetic acid is added, then a fragment of cesium chloride.

If both lead and copper are present in the same solution, both can be tested for in one operation. The solution containing the lead and copper is evaporated to dryness. The residue is taken up in a drop of 1 per cent acetic acid, and successive fragments of sodium acetate and potassium nitrite are introduced into the drop. If the triple nitrite does not form, more 1 per cent acetic acid is added, then a fragment of cesium chloride.

All minerals containing lead tend to form the basic nitrate of lead when evaporated down to $1: 1 \mathrm{HNO}_{3}$. This salt is relatively insollble in 1 per cent acetic acid and in the acetate reagent above described. The procedure recommended by the present writer is as follows: Take the mineral in solution by means of $1: 1 \mathrm{HNO}_{3}$; evaporate to dryness. Leach the residue with a drop of $1: 7 \mathrm{HNO}_{3}$; transfer drop to another place on the slide and slowly evaporate to dryness. Then add acetate reagent or 1 per cent acetic acid and proceed as already described. More or less antimony or bismuth if present will. be carried over with the $1: 7 \mathrm{HNO}_{3}$ solution, but these elements tend to hydrolyze in the weak acetate reagent and do not materially interfere with the test.

Chamot ${ }^{85}$ states:

The addition of an excessive amount of potassium nitrite is objectionable because of the fact that the triple nitrite is quite soluble in solutions of this reagent. On the other hand it is essential that the amount be very slightly in

${ }^{85}$ Chamot, E. M., op. cit., p. 374 . 
excess of that called for by theory. It is therefore necessary to proceed somewhat cautiously. Add a tiny fragment of nitrite; then, after waiting a few moments, if no crystals appear, add a little more.

Nickel can be substituted for copper in the triple nitrite reaction. Before the discovery of the dimethyl glyoxime test for nickel, the triple nitrite test was the one relied upon for the identification of that element. The nickel salt $2 \mathrm{KNO}_{2} \cdot \mathrm{Ni}\left(\mathrm{NO}_{2}\right)_{2} \cdot \mathrm{Pb}\left(\mathrm{NO}_{2}\right)_{2}$ is precipitated as colorless, yellow, and light-brown cubes very much smaller than those of the corresponding copper salt. The color of the nickel salt is also decidedly different from that of the copper salt. The nickel salt never forms dark-brown to black cubes, which are most characteristic of the copper salt.

In the writer's opinion, the triple nitrite reaction is very valuable and should be at the disposal of all who employ microchemical methods. The test requires more skill in manipulation than the iodide test for lead. On the other hand, the triple nitrite is a stable compound and once formed persists afier the drop has dried up, whereas the normal iodide of lead is transitory in character. The delicacy of the triple nitrite test is about equal to that of the iodide test. The triple nitrite works well in the presence of bismuth, in contrast with the iodide test, which is seriously impaired by its presence.

In general the writer prefers the iodide test for lead on account of its simplicity, but in case there is any doubt about the iodide test, the triple nitrite test furnishes a valuable check.

Cesium chloride.-Lead chloride has only a slight solubility in 1:5 HCl. However, a fragment of cesium chloride placed in such a solution will precipitate small but well-formed crystals of the double chloride $\mathrm{PbCl}_{2} \cdot 2 \mathrm{CsCl}$. These are usually short colorless prisms of rectangular outline. They give a low interference color in polarized light and parallel extinction. The quantity of such a precipitate is small, and it does not interfere with tests for antimony, bismuth, tin, and tellurium.

This reaction is not recommended as a test for lead, but the student should be familiar with it, as it is likely to appear when he is testing for other elements.

If successive fragments of $\mathrm{KI}$ and $\mathrm{CsCl}$ are placed in a $1: 5 \mathrm{HCl}$ solution containing lead ions, the double chloride just described usually forms. In addition long, thin yellow to orange-colored needles are usually observed at the edge of the drop. These may be crystals of the double iodide, but this is not certain.

Ammonium bichromate.-A fragment of ammonium bichromate placed in a 1 per cent $\mathrm{HNO}_{3}$ solution containing lead ions precipi. tates a finely divided amorphous yellow powder, which is lead bi. $15122-31-11$ 
chromate. This reaction often appears in testing for silver. It is best to check the presence of lead in the original residue by the potas. sium iodide test. Usually 1 per cent $\mathrm{HNO}_{3}$, will remove only a small proportion of the lead. A drop of 1:5 HCl is applied to the original residue and then evaporated off over the microflame. A drop of water is then applied to the residue and also evaporated off in order to remove the last traces of $\mathrm{HCl}$. The residue is cooled and then leached with a drop of $\mathrm{H}_{2} \mathrm{O}$, and the drop is transferred and tested with a fragment of $\mathrm{KI}$.

\section{SILVER}

A large number of silver minerals occur in nature, but many of them are rare. The most abundant sources of silver, however, are certain copper and lead minerals, such as tetrahedrite, tennantite, and gàlena. Careful microscopic examination of these ore minerals indicates that in most localities the silver is contained in the molecule itself and not as definite silver minerals included in the basemetal minerals.

All the silver minerals tested by the writer are broken down by 1:1 $\mathrm{HNO}_{3}$, but some of them entail considerable difficulty. Pyrargyrite, for instance, usually requires about 10 successive drops of nitric acid, each evaporated to dryness over a microflame, before enough material is available in soluble form for testing. It is exceedingly important that no chlorine ions be introduced, as silver chloride is immediately precipitated and once formed can not be broken down by subsequent treatment with nitric acid. Hence in transferring material from the polished sulphide to the glass slide the match should be moistened with distilled water-not by touching it to the tongue, as the saliva contains enough sodium chloride to precipitate part of the silver when the match is introduced into the test drop. The nitric acid should be tested for its freedom from chlorine ions by pouring a little of it into silver nitrate solution. If any cloudiness appears, the acid should be rejected. These precautions are not necessary if the silver chloride test is used.

\section{Ammonium bichromate test.-}

Reagent: Solid ammonium bichromate $\left(\left(\mathrm{NH}_{4}\right)_{2} \mathrm{Cr}_{2} \mathrm{O}_{7}\right)$.

Product: Silver bichromate in ruby-red triclinic crystals; sometimes also silver chromate in ruby-red orthorhombic crystals.

Limit: $0.02 \% \mathrm{AgNO}_{3}$.

The mineral is dissolved in 1:1 nitric acid. The excess acid is carefully evaporated off, avoiding overheating the residue. The residue is leached with 1 per cent nitric acid. The drop is transferred to a different place on the slide and tested with a fragment of ammonium bichromate by method III. The silver separates as bichromate and chromate. Both compounds are dark ruby-red in 
color. The bichromate is triclinic, and the plates give inclined extinction in polarized light. The chromate is orthorhombic and shows prrallel extinction. The bichromate shows little or no pleochroism, whereas the chromate shows strong pleochroism; thin chromate plates are blood-red when the elongation is parallel to the plane of polarization and yellow when perpendicular to it. Usually the bichromate predominates over the chromate if the silver nitrate solution is pure. In the presence of sulphuric, selenious, or tellurious acids and other extraneous ions the silver tends to precipitate as chromate. The bichromate shows an astonishing variety of forms, a few of which are shown in Plate 7. The color of the crystals and their solubility in ammonia are distinctive criteria. With weaker concentration of silver it is necessary to use a very small fragment of ammonium bichromate-just enough to give part of the drop a faint tinge of yellow.

When the drop has nearly evaporated the yellowish ammonium bichromate residue usually obscures the reddish silver compounds, and it is necessary to add a drop of 1 per cent $\mathrm{HNO}_{3}$ in order to take the excess ammonium bichromate into solution again. Both silver compounds are insoluble in weak nitric acid after they have once formed.

Copper does not interfere with the bichromate test for silver unless it is present greatly in excess of the silver. The writer was unable to get a bichromate test for silver in tennantite which assayed 1 per cent of Ag. Some freibergite specimens, however, gave good bichromate tests for silver. No analyses of these specimens were available. A solution containing 2.5 per cent of $\mathrm{Cu}\left(\mathrm{NO}_{3}\right)_{2}$ and 0.05 per cent of $\mathrm{AgNO}_{3}$ gave a good silver test, but a solution containing 2.5 per cent of $\mathrm{Cu}\left(\mathrm{NO}_{3}\right)_{2}$ and 0.025 per cent of $\mathrm{AgNO}_{3}$ gave a negative silver test.

Lead is precipitated as chromate, usually as an amorphous yellow powder. It does not prevent the formation of the red silver bichromate and chromate crystals, but an abundance of the yellow powder may mask the observations of the silver crystals. A solution containing 0.5 per cent of $\mathrm{Pb}\left(\mathrm{NO}_{3}\right)_{2}$ and 0.05 per cent of $\mathrm{AgNO}_{3}$ gave a good bichromate test for silver. None of the specimens of argentiferous galena tested by the writer yielded a bichromate test for silver.

The ammonium bichromate test for silver is highly distinctive, and when the concentration of silver is comparatively high the test is almost spectacular. On the other hand, certain solutions seem to reduce the sensitivity of the test greatly. Also it requires considerable sill and experience to get the conditions just right. One source of error, getting chloride ions into the test drop, has already been mentioned. Another error commonly made by beginners is mistaking 
ammonium bichromate itself for the silver compound. The ammo. nium bichromate is orange-colored and very soluble, whereas the sil. ver compound is red and once formed is highly stable in 1 per cent $\mathrm{HNO}_{3}$. To make certain that the observer sees the silver compound, he should allow the drop to evaporate almost to dryness, then add a drop of 1 per cent $\mathrm{HNO}_{3}$ to the residue. The ammonium bichromate goes back into solution, and any silver bichromate and chromate crystals present are not affected.

Again it should be emphasized that when the concentration of silver is low (less than 0.1 per cent of $\mathrm{AgNO}_{3}$ ) the crystals of silver bichromate will be very small, needlelike, but usually irregular, and will form at the edge of the drop. (See pl. 7, D.) The color of these crystals is the main criterion. If the crystals are very small, the color may best be observed in obliquely reflected light.

The student should make a 0.02 per cent $\mathrm{AgNO}_{3}$ solution in 1 per cent $\mathrm{HNO}_{3}$ and prove his skill by making successive tests until consistent positive results are obtained.

Chloride test.-

Reagents: $1: 5 \mathrm{HCl}$ and concentrated $\mathrm{NH}_{4} \mathrm{OH}$.

Product: Silver chloride in minute colorless isometric crystals.

Limit: $0.02 \% \mathrm{AgNO}_{3}$.

The mineral is broken down chemically by successive drops of 1: $1 \mathrm{HNO}_{3}$. A drop of 1: $5 \mathrm{HCl}$ is applied to the dried residue. Any silver present is immediately precipitated as chloride. Silver chloride is so insoluble in water and in acids that it is completely amorphous. It can be made to crystallize by dissolving in concentrated ammonia and allowing the solution to evaporate. As evaporation proceeds tiny highly refracting colorless isometric silver chloride crystals begin forming at the periphery of the drop, and precipitation proceeds toward the center until the drop dries up. If the concentration in silver is comparatively high, the crystals will assume complex forms, such as combinations of cube and hexoctahedron, cube and octahedron, and, more rarely, the tetrahedron. Viewed with a 4-millimeter objective many of these crystals are very beautiful. Often triangular and pseudohexagonal plates can be observed. When the concentration of silver is comparatively low more simple forms predominate. The cube is the most characteristic form, but the octahedron is frequently formed.

Some elements have a detrimental effect on the test, notably coppar and tellurium. Copper causes the precipitate to come down as very small black skeletal crystals. When these appear copper should be tested for. If found, the silver test should be repeated, and the silver chloride should be leached with successive drops of $1: 7 \mathrm{HNO}_{3}$ until the drop fails to give a copper test with potassium mercuric 
thiocyanate. Then the silver chloride should be leached with a drop of water to remove the nitric acid and dissolved in ammonia.

If tellurium is present, the silver chloride should be leached by successive drops of $1: 5 \mathrm{HCl}$, and each drop removed and tested by megns of a fragment of $\mathrm{CsCl}$. When the drop does not give an immediate precipitate with cesium chloride, the silver chloride is sufficiently free from tellurium to proceed with the silver test. It is then washed with a drop of water and taken into solution with $\mathrm{NH}_{4} \mathrm{OH}$.

Mercurous mercury and lead are the other common elements of group I (qualitative analysis), which are precipitated by chlorine ions. Neither dissolves in ammonia. Mercurous chloride turns black; lead chloride is converted to the insoluble hydrate, usually retaining the needle forms of the lead chloride pseudomorphously. Formerly the writer preferred to leach the silver chloride with ammonia and transfer the ammonia drop to another place on the slide for observation. It has been found better to leave the ammonia drop on the original residue; the crystals seem to be larger and better formed in this manner. To avoid confusion between the white particles of the original residue and the silver chloride crystals the ammonia drop is made large enough to spread out a little beyond the edge of the residue. Then the microscope is trained on the edge of the ammonia drop until the first crystals of silver chloride form.

A specimen of tennantite from Bonanza, Colo., was analyzed and found to contain 1.00 per cent of Ag. It was tested in the manner above described and yielded well-formed cubes of $\mathrm{AgCl}$. However, the writer was not able to obtain a satisfactory chloride test for silver by making up a solution containing 0.5 per cent each of $\mathrm{Cu}\left(\mathrm{NO}_{3}\right)_{2}$ and $\mathrm{AgNO}_{3}$ in $\mathrm{H}_{2} \mathrm{O}$. The silver yielded an abundant amorphous white precipitate when $\mathrm{HCl}$ was added to the solution. This precipitate was washed with five successive drops of $1: 7 \mathrm{HNO}_{3}$ and one of water. When dissolved in $\mathrm{NH}_{4} \mathrm{OH}$, the forms reprecipitated were small, black, and skeletal. The writer can not explain this discrepancy.

Mixtures of $\mathrm{Pb}\left(\mathrm{NO}_{3}\right)_{2}$ and $\mathrm{AgNO}_{3}$ were tested by this method. The lead chloride occludes some of the silver chloride formed and 80 reduces the sensitivity of the test somewhat, although not seriously. A solution containing 0.5 per cent of $\mathrm{Pb}\left(\mathrm{NO}_{3}\right)_{2}$ and 0.05 per cent of $\mathrm{AgNO}_{3}$ gave well-formed cubes. One containing 0.5 per cent of $\mathrm{Pb}\left(\mathrm{NO}_{3}\right)_{2}$ and 0.025 per cent of $\mathrm{AgNO}_{3}$ gave a negative test. Several specimens of galena associated with silver minerals were tested in the same way, but all of them gave negative silver tests.

It frequently happens that in making this test minute black formless grains come down when the ammonia drop evaporates. Com- 
monly these are probably silver chloride grains, but in the absence of definite isometric forms it is safest to consider the test indecisive.

The silver chloride test does not give the satisfaction that the bichromate test affords. The crystals are small and colorless. However, a little experience with this method imparts a surprising amount of confidence in it, owing to the unique chemical behavior of silver chloride. The test is more sensitive than the bichromate test, but its chief value lies in the fact that silver can be separated from elements, notably lead, that interfere with the bichromate test. Fa. miliarity with the chloride test is therefore recommended, although for most purposes the bichromate test is best.

A curdy white precipitate suddenly appearing when a drop of $\mathrm{HCl}$ is introduced in the course of a microchemical test gives a decided indication of the presence of silver. This precipitate should be cleaned by suitable acids and further tested by means of ammonia.

\section{Potassium mercuric thiocyanate test.-}

Reagents: $5 \% \mathrm{~K}_{2} \mathrm{Hg}(\mathrm{CNS})_{4}$ in $\mathrm{H}_{2} \mathrm{O}$.

Product: Curdy white amorphous precipitate (KCNS.AgCNS).

Limit: $0.02 \% \mathrm{AgNO}_{8}$.

The mineral is broken down with $1: 1 \mathrm{HNO}_{3}$. The residue is leached with a drop of $1: 7 \mathrm{HNO}_{3}$, the drop transferred to another place on the slide and joined to a drop of potassium mercuric thiocyanate (method I). An amorphous cloudy white precipitate forms. 'This reaction is useful in that the precipitate often appears incidentally in tests for other elements. So far as the writer is aware no other element gives a similar precipitate with potassium mercuric thiocyanate. However, when it appears, the presence of silver should be confirmed by the bichromate test.

The potassium mercuric thiocyanate test for silver works particularly well in the presence of copper. The silver precipitate comes down before the copper precipitate and may be seen as a horseshoeshaped wave of white grains spreading out in front of the copper precipitate. The silver precipitate is best seen in obliquely reflected light.

\section{GOLD}

Gold minerals in nature are few. The most common is the native metal itself. It is readily recognized in polished surfaces by its color and sectility. An etching test with KCN will serve to distinguish gold from chalcopyrite in case the area is too small to scratch.

Tellurium is the element that is most frequently found in goldbearing minerals-in fact, it is almost the only element which is found in nature combined with gold. A selenide of gold is known but is exceedingly rare. There are a number of tellurides of gold, 
U. S. GEOLOGICAL SURVEY
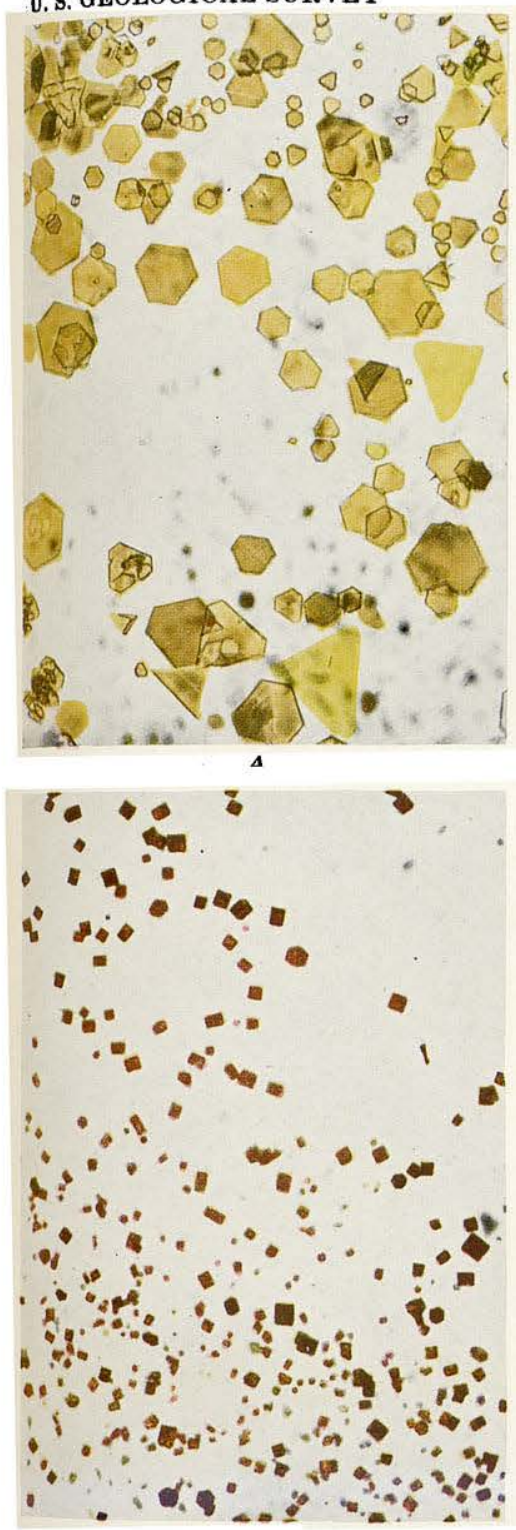

C
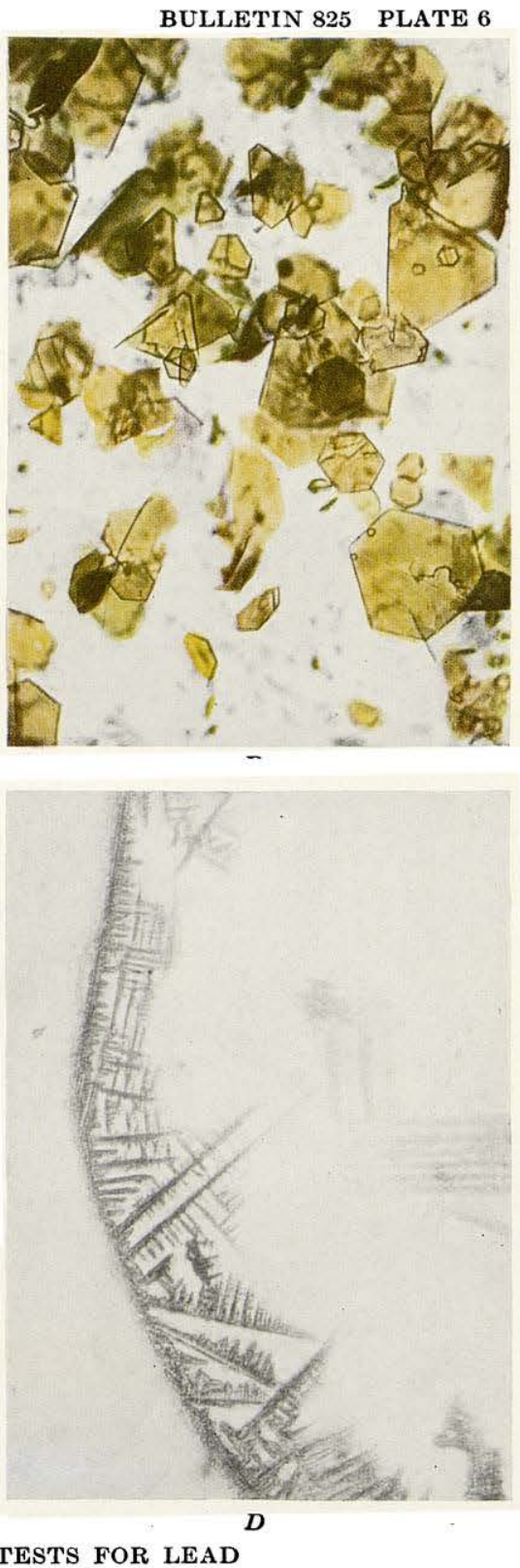

$A$, Lead iodide crystals same solution as $A$. C. Potassium-lead-copper triple nitrite crystals. Obtained from a $0.5 \%$ lead acetate solution. $\times 215$. $D$, Lead nitrate residue. Obtained from evaporating to dryness a drop of $1: 1 \mathrm{HNO}_{3}$ containing galena powder. $\times 70$. 

U.S. GEOLOGICAL SURVEY
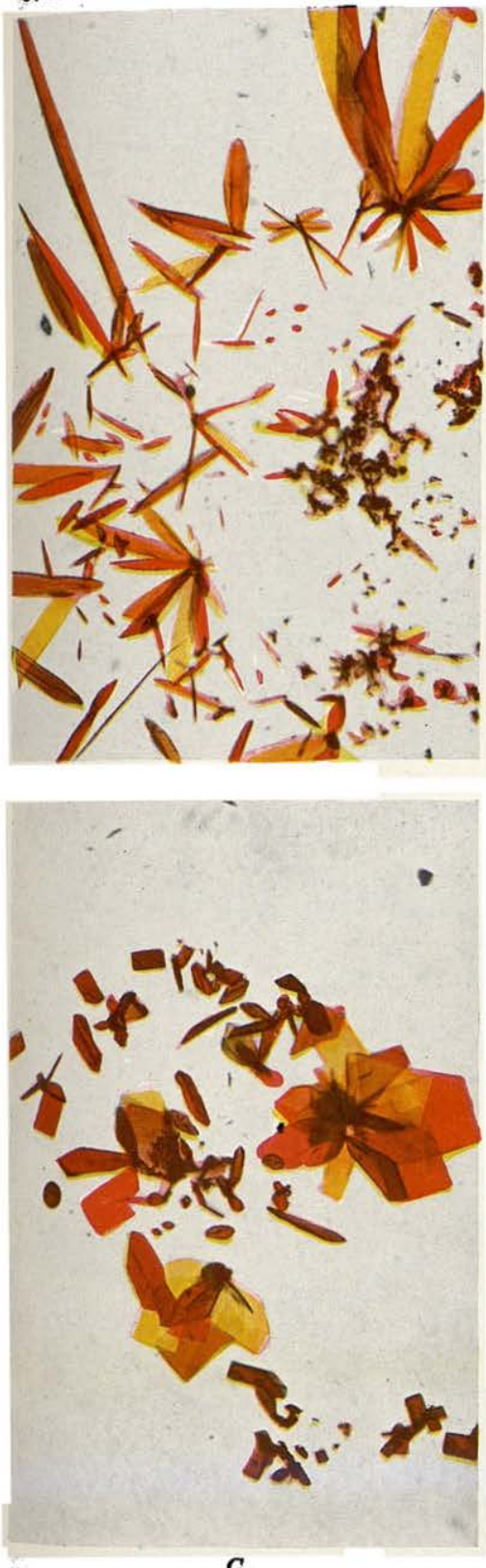

BULLETIN 825 PLATE 7
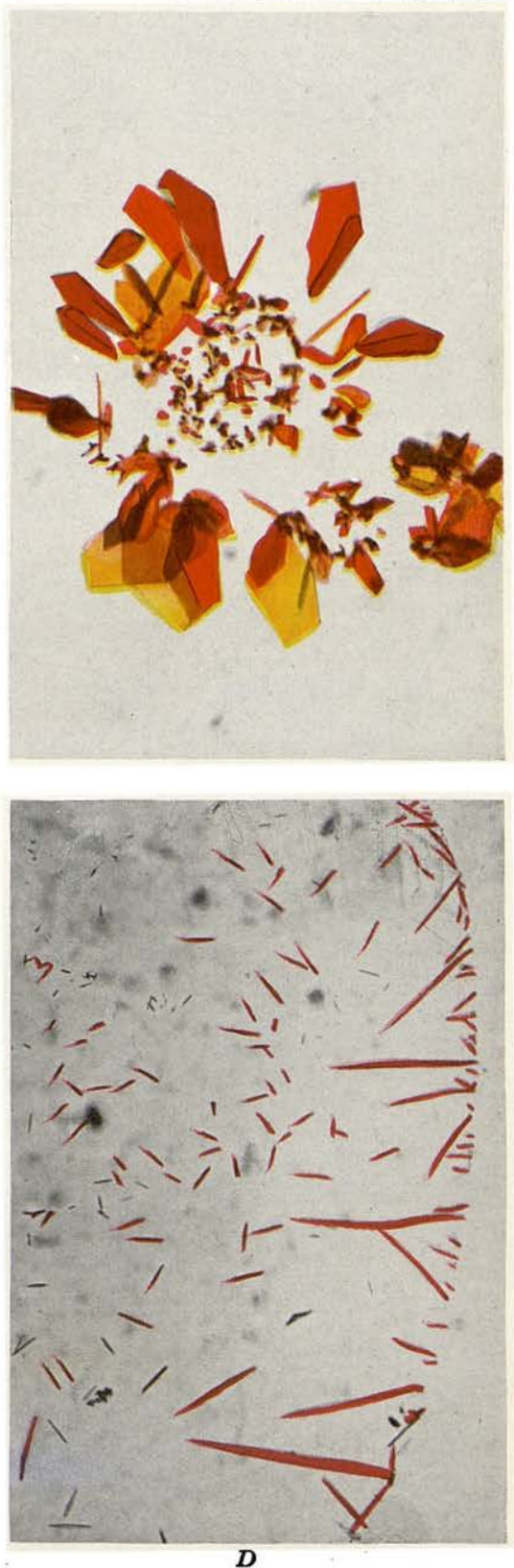

A, Silver bichromate crystals. Obtained from a $0.5 \%$ silver nitrate solution. $\times 70$. $B, C$, From same solution as $A$. $D$, Silver bichromate crystals. Obtained from a $0.1 \%$ silver nitrate solution. $\times \mathbf{7 0}$. 


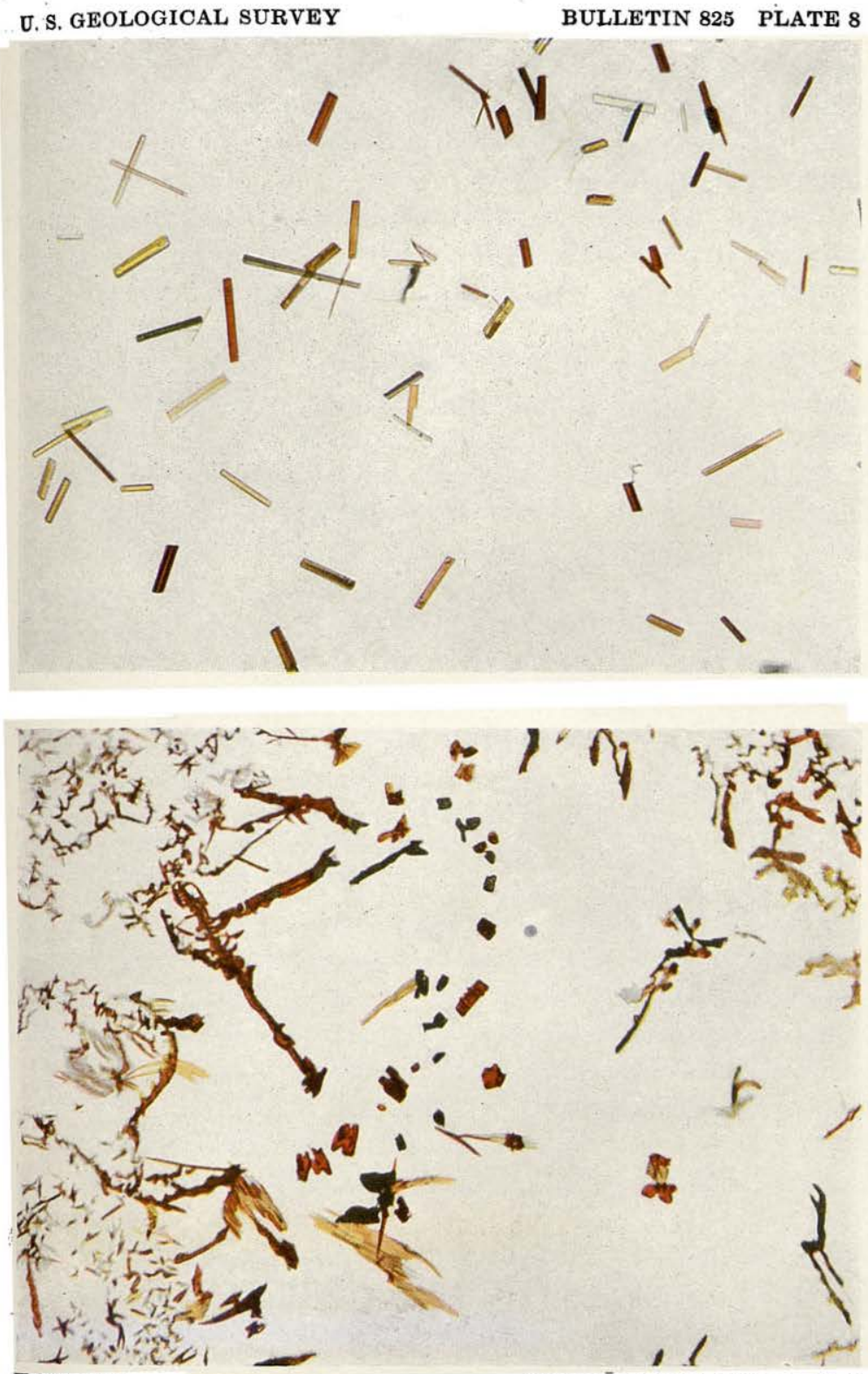

Halftone by

The Colorplate Engraving Co., New York, N. Y., Roberts, and D. Selchow.

CRYSTALS OF GOLD PYRIDINE DOUBLE BROMIDE $\times 250$. Agfa color plate 

most of them containing more or less silver. These minerals are not characteristic in appearance, and it is usually desirable to confirm their identity by microchemical tests.

The tellurides are easily broken down by 1:1 $\mathrm{HNO}_{3}$. The dense white amorphous tellurious acid and metallic gold remain. The gold is usually covered and obscured by the tellurious acid. This acid is readily soluble in 1:5 HCl, however, and the gold can then be recognized by its color by observing it in obliquely reflected light. A No. 3 Leitz or other objective of equivalent magnification is most satisfactory for this purpose. This suffices to identify gold in the majority of tests.

Where a confirmatory test is desirable, the gold can be taken into solution by means of aqua regia. Before doing this it is best to make sure that all the tellurium is removed. The original residue should be leached by successive drops of $1: 5 \mathrm{HCl}$ and each drop tested by means of a fragment of cesium chloride until a precipitate is not immediately thrown down. (See p. 165.) A drop of aqua regia is then applied and the gold dissolved. The residue is gold chloride $\left(\mathrm{HAuCl}_{4}\right)$ and is orange-colored and very soluble in water. A drop of $\mathrm{H}_{2} \mathrm{O}$ is applied to this residue. When the residue is dissolved the drop is transferred to another place on the slide and tested by one of the two following methods.

\section{Pyridine double bromide test.-}

Reagent: 1 volume pyridine in 9 volumes of $40 \% \mathrm{HBr}$.

Product: $\left(\mathrm{Cl}_{6} \mathrm{H}_{6} \mathrm{NH}\right) \mathrm{AuBr}_{4}$; pleochroic crystals.

Limit: $0.02 \% \mathrm{HAuCl}_{4} .4 \mathrm{H}_{2} \mathrm{O}$ solution.

The most satisfactory test for gold is the formation of the double bromide of gold and pyridine. Credit for this test is due to Messrs. Putnam, Roberts, and Selchow, who discovered and described it. (See p. 116.) The gold is taken into solution by one or more applications of aqua regia, and the excess acid is evaporated off. The residue is leached with a drop of water, and the drop is transferred to another place on the slide and joined to a drop of the reagent by method I. A well-crystallized precipitate forms if gold is present in sufficient concentration.

The crystal form varies somewhat with the concentration. With moderately concentrated solutions prisms predominate. With dilute solutions the crystals are leaflike and feathery, often radiating inward from the periphery of the drop. (See pl. 8.)

The crystals are extraordinarily pleochroic, varying from orangecolored to maroon when the elongation is perpendicular to the vibration direction of the lower nicol and from colorless to pale yellow for the parallel orientation. This extreme pleochroism is diagnostic of the compound. The crystals are monoclinic or triclinic, and the maximum extinction is about $10^{\circ}$ on the prism. 
The authors cited state that the following elements give no reactions themselves within 10 minutes from 0.02 molal solution, nor does their presence singly or together appreciably affect the formation of the gold compound: $\mathrm{Al}, \mathrm{As}, \mathrm{Ba}, \mathrm{Be}, \mathrm{Bi}, \mathrm{Ca}, \mathrm{Cd}, \mathrm{Cl}, \mathrm{Co}, \mathrm{Cr}, \mathrm{Cs}$, $\mathrm{Cu}, \mathrm{Fe}, \mathrm{Ga}, \mathrm{Ge}, \mathrm{Hg}$ (mercuric), I, In, Ir, La, Mg, Mn, Mo, Ni, Os, $\mathrm{PO}_{4}, \mathrm{Pd}, \mathrm{Pt}, \mathrm{Ru}, \mathrm{Se}, \mathrm{Sn}$ (stannic), Te, $\mathrm{Th}, \mathrm{Ti}, \mathrm{UO}_{2}, \mathrm{~V}, \mathrm{~W}, \mathrm{Zn}$, and, by analogy based on the periodic system, B, Li, Na, K, Rb, Sr, Sb.

Under ordinary conditions the analogous lead compound may also be precipitated. This compound is characterized by long, grasslike, colorless, nonpleochroic crystals, and hence differs widely from the corresponding gold compound. Most of the lead remains behind as chloride when the residue from the original aqua regia treatment is leached with water. The authors state that the lead can be kept in solution by adding $\mathrm{NaBr}$ to the test drop before joining the reagent drop to it.

This gold test is particularly valuable in that the presence of tellurious acid does not interfere with it. The limit of dilution as determined by the present writer is about 0.02 per cent of gold chloride.

Experiments carried out on lead nitrate solutions of various concentrations indicate that the pyridine and $\mathrm{HBr}$ solution will cause precipitate from a 0.05 per cent $\mathrm{Pb}\left(\mathrm{NO}_{3}\right)_{2}$ or more concentrated solution. The precipitate is not sufficiently characteristic in appearance and properties to constitute a good lead test, but the student should familiarize himself with this compound, as it may appear when he is testing for gold.

Stannous chloride test.-

Reagent: $5 \% \mathrm{SnCl}_{2}$ in 1:5 $\mathrm{HCl}$.

Product: Metallic gold (purple of Cassius).

Limit: $0.1 \%$ gold chloride.

The mineral is dissolved in 1:1 $\mathrm{HNO}_{3}$. Usually metallic gold separates out and may be recognized by its color. If necessary, however, a confirmatory test can be made as follows: Dissolve the residue in aqua regia. The reddish-brown trichloride is formed. Dissolve this in a drop of water and transfer the drop. Test the drop with stannous chloride solution, using method I. A precipitate of finely divided metallic gold forms where the solutions come into contact. This spreads out toward the periphery of the test drop in a widening band. The precipitate is amorphous and ranges in color from reddish to bluish tinges. Purple is the most distinctive color (purple of Cassius).

Under similar conditions selenious acid yields a red precipitate and tellurious acid a dull-black powder. It must be admitted that this test leaves much to be desired as regards delicacy and uniform- 
itt in appearance of the precipitate. It works in the presence of other elements, however, and is given here because the reaction may appear in testing for selenium. All traces of tellurious acid must be removed before testing for gold.

The thallous nitrate reaction described by Behrens and Kley is unreliable and should be avoided.

\section{ARSENIC}

The reactions for arsenic recommended in the textbooks on microchemistry can be summarized as follows:

1. The production of arsine. This test is highly recommended by Chamot and is an adaptation of the familiar Marsh test for arsenic used in qualitative chemical analysis. It is undoubtedly exceedingly sensitive but requires a somewhat complicated apparatus ${ }^{86}$ and is therefore not used in this work, as the ammonium molybdate test described below is highly satisfactory and can be made on a glass slide.

2. The formation of silver arsenate. The red crystals formed by adding silver nitrate to an arsenate solution are characteristic, but the test solution must be nearly neutral, and, moreover, many other dements interfere with this test.

3. The formation of ammonium-magnesium arsenate. This compound is the one commonly used in quantitative analysis. It is recommended by Behrens and Kley as a microchemical test. A grain of magnesium acetate is added to an ammoniacal arsenate solution. The crystals formed are colorless but are characteristic in shape. ${ }^{87}$ It is the writer's experience that this test requires conditions restricted in range. Although it is sensitive and was obtained on a 0.01 per cent ammonium arsenate solution, the crystals so formed mere blunt square prisms rather than the more distinctive envelopeshaped crystals depicted by Behrens and Kley, which were obtained only in relatively concentrated solutions. The test requires alkaline conditions and is therefore less convenient than one that will work in acid solutions.

4. The formation of ammonium arseni-molybdate. This test is the same as the corresponding phosphorus test used in qualitative analysis. According to Behrens and Kley it is far inferior to the ammonium-magnesium arsenate test just described, but the writer's experience is decidedly to the contrary. The molybdate test is entirely satisfactory and is recommended for the following reasons: It is at least as sensitive as the ammonium-magnesium arsenate test and gives a good reaction with 0.01 per cent ammonium or

"Chamot, E. M., op. cit., fig. 162, p. 396.

"Behrens, H., and Kley, P. D. C., op. cit., p. 136. fig. 93. 
sodium arsenate; it is effective in strongly acid solutions; the crystals obtained are yellow in color and characteristic in shape; only two elements (phosphorus and silicon) interfere with the test; the test works over a great range of conditions and is almost "foolproof." Ammonium molybdate test.-

Reagent: 1.5\% ammonium molybdate in 1: $7 \mathrm{HNO}_{3}$.

Product: Ammonium arseni-molybdate in tiny yellow isometric crystals. Limit: $0.01 \%\left(\mathrm{NH}_{4}\right)_{3} \mathrm{AsO}_{4}$.

The mineral is decomposed on a glass slide by successive drops of 1:1 $\mathrm{HNO}_{3}$ or aqua regia. Arsenic compounds in general are more easily broken down by aqua regia than by nitric acid, but if antimony is also to be tested for it is better to use nitric acid. Both acids have a strongly oxidizing action and convert arsenic to the pentavalent state.

The residue is leached with a drop of $1: 7 \mathrm{HNO}_{3}$. Antimony remains behind; arsenic goes into solution as arsenic acid, $\mathrm{H}_{3} \mathrm{As}_{4} \mathrm{O}_{4}$. The drop is transferred to a new place on the slide and slowly evaporated over the microflame. When nearly dry a drop of the 1.5 per cent ammonium molybdate solution is added to the residue. This is evaporated very slowly by holding the slide at least 4 inches above the microflame and drawing it in and out of the heat until evaporation is complete. When dry, the excess ammonium molybdate is dissolved by adding a drop of $1: 7 \mathrm{HNO}_{3}$ to the residue. Tiny yellow octahedra of ammonium arseni-molybdate remain if arsenic is present inthe unknown solution. (See pl. 9, $C, D$.)

These crystals are isometric. Octahedra predominate, but usually other isometric forms such as the trisoctahedron are combined with the octahedron. The crystals are usually very small and are often so nearly spherical that the individual forms can not be distinguished. Twins, especially on the spinel law, are common, and tiny crosses seem to suggest other systems than the isometric. The crystals are, however, isotropic in polarized light. The color is distinctive-a light lemon-yellow-and is best observed in oblique light, using a No. 4 Leitz or 8-millimeter Bausch \& Lomb objective and turning aside the reflecting mirror so as to cut off the transverse illumination. The crystals are readily soluble in $\mathrm{NH}_{4} \mathrm{OH}$ but are not easily recrystallized.

Ammonium arseni-molybdate is precipitated slowly and only partly in cold solutions, in contrast to the corresponding phosphorus compound, which is precipitated rapidly and completely under the same conditions. Few if any ore minerals having a metallic luster contain phosphorus.

Bismuth, according to Behrens and Kley, will form a precipitate identical in appearance with the corresponding arsenic compound. 
The writer can not confirm this statement. Tests carried out on different concentrations of bismuth nitrate, using the same procedure as the test for arsenic, failed to give any precipitate.

Silicic acid is completely removed by evaporating the mineral to dryness with $\mathrm{HNO}_{3}$; insoluble $\mathrm{SiO}_{2}$ separates out of the solution.

The ammonium molybdate test for arsenic does not interfere with the usual tests for other elements. For instance, after the arsenic test already outlined is made, the unknown solution can be tested for cobalt or copper by placing a drop of potassium mercuric thiocyanate directly on top of the 1:7 $\mathrm{HNO}_{3}$ solution containing the excess of ammonium molybdate. (Method III-A, p. 125.) In fact, the copper or cobalt mercuric thiocyanate forms better crystals when arsenic has been removed from solution than it does when arsenic is present. Antimony and arsenic are common associates but are easily separated by means of nitric acid. In testing for arsenic, it is customary to test for antimony as well. If bismuth is present it will be indicated in the test for antimony.

To throw down ammonium arseni-molybdate there must be a considerable excess of ammonium molybdate. However, if too much ammonium molybdate is present some will separate out in advance of the arseni-molybdate and obscure it. For this reason it is preferable to use an ammonium molybdate solution of definite strength rather than to introduce the solid reagent. The test works in acid or neutral solutions, but the ammonium arseni-molybdate crystals are most characteristic when formed in a nitric acid solution. The writer has found by experiments with solutions of various strengths that a 1.5 per cent solution of ammonium molybdate in 1:7 $\mathrm{HNO}_{3}$ forms the most satisfactory reagent.

\section{Potassium iodide test.-}

Reagent: Solid KI.

Product: Arsenic tri-iodide ( $\mathrm{AsI}_{3}$ ), an amorphous yellow powder.

Limit: $0.02 \%\left(\mathrm{NH}_{4}\right)_{3} \mathrm{AsO}_{4}$ in $1: 5 \mathrm{HCl}$.

The mineral is broken down by metuns of $1: 1 \mathrm{HNO}_{3}$ or aqua regia. The residue is leached with a drop of $1: 5 \mathrm{HCl}$, and the drop transferred to another place on the slide. A fragment of $\mathrm{KI}$ is placed in the drop. An amorphous yellow to orange-colored precipitate forms if arsenic is present. The color of the arsenic precipitate resembles that of the copper iodide precipitate but is a darker shade of yellow. The color is best observed in obliquely reflected light, with a 16millimeter objective.

With solutions of greater concentration than 0.10 per cent $\left(\mathrm{NH}_{4}\right)_{3} \mathrm{AsO}_{4}$ the precipitation is immediate; with lesser concentration, the precipitate comes down at the edge of the drop a few seconds later. 
Under the same conditions antimony colors the drop yellow but gives no precipitate.

The iodide test is not as distinctive as the molybdate test for arsenic but is very sensitive and is frequently encountered in making the cesium double iodide test for antimony or bismuth.

Cesinom double iodide test.-

Reagents: Solid KI and CsCl.

Product: Arsenic cesium double iodide ( $\mathrm{AsI}_{3} .3 \mathrm{CsI}$ ) in orange-colored hexagons or formless grains.

Limit: $0.02 \%\left(\mathrm{NH}_{4}\right)_{3} \mathrm{AsO}_{4}$ in 1:5 $\mathrm{HCl}$.

The mineral is broken down by $1: 1 \mathrm{HNO}_{3}$ or aqua regia. The residue is leached with a drop of 1:5 HCl, and the drop transferred. A fragment of $\mathrm{KI}$ is added, then one of $\mathrm{CsCl}$. The $\mathrm{KI}$ gives an amorphous precipitate, as described in the preceding test. When the $\mathrm{CsCl}$ fragment is added the arsenic iodide precipitate is replaced by a deep orange-colored precipitate which is probably the cesium arsenic double iodide. This is usually amorphous, but if the concentration in arsenic is relatively high, orange-colored hexagons will sometimes form in addition to the amorphous precipitate. These hexagons are identical in shape and color with those of the corresponding antimony compound. (See p. 161.) This would apparently be an exceedingly good test for arsenic were it not for the danger of confusing it with the antimony test.

In contrast with antimony, cesium chloride alone does not give a precipitate with arsenic, hence in testing for antimony it would be better to add the cesium chloride before the potassium iodide. The fact that potassium iodide alone precipitates arsenic out of an $\mathrm{HCl}$ solution is also of great value in distinguishing between arsenic and antimony. When the yellow arsenic iodide precipitate appears, the original residue should be leached with successive drops of 1:7 $\mathrm{HNO}_{3}$ until all the arsenic is removed before testing for antimony. When the arsenic is removed from the original residue, this can be tested for antimony by leaching with a drop of $1: 5 \mathrm{HCl}$. This is transferred and a fragment of $\mathrm{KI}$ added. If a yellow amorphous precipitate forms, arsenic is still present and the purification is not complete. If no precipitate forms and the solution stains yellow, the presence of antimony is indicated. It may be confirmed by adding a fragment of $\mathrm{CsCl}$. Orange-colored hexagons will then prove antimony and not arsenic. If no yellow precipitate appears when $\mathrm{KI}$ is added and the solution does not turn yellow, antimony can not be tested for by the cesium double iodide method, because orange-colored hexagons would mean either arsenic or antimony. The process should be repeated, using the cesium double chloride test for antimony (p. 160). 


\section{These facts are summarized as follows:}

KI alone gives an amorphous yellow precipitate with arsenic.

KI alone gives no precipitate with antimony. It colors a moderately concentrated antimony solution light yellow but is without effect on a dilute solution.

$\mathrm{CsCl}$ alone gives no precipitate with arsenic.

$\mathrm{CsCl}$ alone gives a well-defined precipitate with antimony.

The cesium double iodide reaction with arsenic is not mentioned in the literature. For reasons already given it is regarded as a nuisance by the present writer. However, a complete understanding of it is of vital importance when dealing with arsenic and antimony minerals.

\section{ANTIMONY AND BISMUTH}

The microchemical tests for antimony and bismuth are so similar that some experience is necessary to distinguish between them. If either element is in the solution to the exclusion of the other, no difficulty is experienced in its identification. If both elements are present, however, they tend to form isomorphous crystals. Hence it is believed best to describe the two elements together.

Bismuth compounds are readily soluble in $\mathrm{HNO}_{3}$, with the formation of the nitrate $\mathrm{Bi}\left(\mathrm{NO}_{3}\right)_{3}$. This will remain in solution in even weak concentrations of $\mathrm{HNO}_{3}$, but in the absence of acids it is hydrolyzed by $\mathrm{H}_{2} \mathrm{O}$, precipitating the oxy-nitrate $\mathrm{BiONO}_{3}$. These bismuth compounds are readily soluble in $1: 5 \mathrm{HCl}$, with the formation of $\mathrm{BiCl}_{3}$. The chloride is likewise hydrolyzed by pure water and precipitates $\mathrm{BiOCl}$. In testing for bismuth the ore mineral containing it is first broken down by $1: 1 \mathrm{HNO}_{3}$. The bismuth nitrate resulting is taken into solution by $1: 5 \mathrm{HCl}$, as the microchemical tests to be described work better in a hydrochloric than in a nitrate solution.

Antimony differs from bismuth in that its compounds are not readily soluble in nitric acid. Antimony compounds, however, are almost without exception easily broken down by 1:1 $\mathrm{HNO}_{3}$, the antimony being oxidized to $\mathrm{Sb}_{2} \mathrm{O}_{5}$. This is a white powder comparatively insoluble in $1: 7 \mathrm{HNO}_{3}$ but unfortunately sufficiently soluble to give a microchemical test for antimony. Therefore nitric acid can not be relied on to separate antimony and bismuth in the residue obtained by evaporating a compound containing the two elements down to dryness with 1:1 $\mathrm{HNO}_{3} . \mathrm{Sb}_{2} \mathrm{O}_{5}$ is readily soluble in 1:5 HCl. Usually not all the antimony goes into solution in the first drop; successive drops can be applied to the same residue and an antimony test obtained from each. Hence if too much antimony is present in the first drop to give a satisfactory test, as shown by a dense amorphous precipitate, the second or third drop of $1: 5 \mathrm{HCl}$ applied to the origi- 
nal residue will usually exhibit well-formed crystals when tested in the same way.

The microchemical tests recommended for antimony and bismuth are $(a)$ the formation of the double chloride with cesium $\left(\mathrm{SbCl}_{8} \cdot 3 \mathrm{Cs} \mathrm{Cl}\right.$ or $\left.\mathrm{BiCl}_{3} .3 \mathrm{CsCl}\right)$ and $(b)$ the formation of the double iodide with cesium $\left(\mathrm{SbI}_{3} \cdot \mathrm{CsCl}\right.$ or $\left.\mathrm{BiI}_{3} \cdot \mathrm{CsCl}\right)$. Both tests have their advantages and disadvantages. If both antimony and bismuth are present in the same compound it is recommended that both tests be made so that the good points of each test can be combined.

\section{Dowble chloride test.-}

Reagents: Solid cesium chloride ( $\mathrm{CsCl})$.

Product: Antimony (or bismuth) cesium chloride $\left(\mathrm{SbCl}_{3} .3 \mathrm{CsCl}\right)$ in colorless highly refracting plates.

Limit: $0.02 \% \mathrm{SbCl}_{3}$ solution; $0.02 \% \mathrm{BiCl}_{3}$ solution.

The mineral is broken down by placing it in a drop of $1: 1 \mathrm{HNO}_{3}$ and evaporating to dryness. The residue is leached in a drop of $1: 5$ $\mathrm{HCl}$, the drop is transferred to another place on the slide, and a fragment of $\mathrm{CsCl}$ introduced into the solution (method III).

Antimony forms star-shaped crystals, also hexagonal plates, some with radiating spokes. The forms exhibited are identical in shape with those of the double iodide. (See p. 162.) Those of the double iodide are strongly colored, however, whereas the double chloride crystals are transparent and colorless. Most of the double chloride precipitate is amorphous. The best-formed crystals are found at the edge of the mass, the center of the mass usually comprising formless, more or less equidimensional grains.

Bismuth forms transparent rhombs that resemble cleavage flakes of calcite. (See pl, 11, C.) In addition to the rhombs, petal-shaped and knifeblade-shaped crystals sometimes form, more rarely hexagons resembling the corresponding antimony compound. In solutions containing bismuth, however, hexagons have never been observed alone, except with very dilute bismuth chloride solutions (0.02 per cent or less $\mathrm{BiCl}_{3}$ ). Almost invariably rhombs are also present.

Bismuth cesium chloride invariably forms very thin transparent plates. They are so thin that they show little or no birefringence in polarized light. In fact, at times they are almost invisible in ordinary light but are readily detected owing to their refringence. By raising and lowering the objective the Becke line becomes prominent. The plates are highly reflecting, however, and sometimes exhibit iridescent colors owing to interference between the waves reflected from top and bottom surfaces. This is marked in polarized light, and the effect has been mistaken for birefringence. It disappears when oblique light is shielded from the slide by means of the hand or other object. The bismuth compound has a much stronger crystallizing tendency than the corresponding antimony compound, and amorphous grains are rare. 
To summarize the above observations, colorless hexagons, stars, and formless grains indicate antimony; very thin, colorless rhombs, petals, and blades indicate bismuth.

When both antimony and bismuth are present the subordinate element combines with part of the predominant element to form isomorphous crystals. The remainder of the predominant element forms the crystals characteristic of that element. The isomorphous crystals have characteristics foreign to both of the constituent elements. Solutions of antimony chloride in $1: 5 \mathrm{HCl}$ and bismuth chloride in 1:5 HCl were mixed in different proportions and tested with $\mathrm{CsCl}$, with the following results:

$0.5 \% \mathrm{SbCl}_{3}$ and $0.01 \% \mathrm{BiCl}_{3}$.-Antimony hexagons and formless grains. No signs of bismuth.

$1.5 \% \mathrm{SbCl}_{3}$ and $0.02 \% \mathrm{BiCl}_{3}$. - Same as preceding.

$.0 .5 \% \mathrm{SbCl}_{3}$ and $0.03 \% \mathrm{BiCl}_{3}$.-Antimony stars, hexagons, and formless grains. No signs of bismuth.

$0.5 \% \mathrm{SbCl}_{3}$ and $0.06 \% \mathrm{BiCl}_{3}$. - Star-shaped crystals, some of considerable size. These undoubtedly contain both $\mathbf{S b}$ and $\mathbf{B i}$, as they are not characteristic of either.

$105 \% \mathrm{SbCl}_{3}$ and $0.12 \% \mathrm{BiCl}_{3}$. - Beautiful star-shaped crystals with branching arms.

$0.5 \% \mathrm{SbCl}_{3}$ and $0.25 \% \mathrm{BiCl}_{3}$. - Same as preceding; also some large white hexagons with ribs.

$0.5 \% \mathrm{SbCl}_{5}$ and $0.5 \% \mathrm{BiCl}_{3}$-Complex star-shaped and treelike crystals.

${ }_{125 \%} \% \mathrm{SbCl}_{3}$ and $0.5 \% \mathrm{BiCl}_{3}$.-Same as preceding; also characteristic bismuth thombs.

$012 \% \mathrm{SbCl}_{3}$ and $0.5 \% \mathrm{BiCl}_{3}$. - Rhombs very abundant; stars less so. Many of the crystals are seedlike, irregular grains.

$0.06 \% \mathrm{SbCl}_{3}$ and $0.5 \% \mathrm{BiCl}_{3}$.- Rhombs abundant; "seeds" less so. The " seeds" are highly refracting and have a heavy black line around them. Some of them are better formed and seem to be flat-lying hexagonal prisms. Irregular forms are abundant.

${ }_{0.3 \%} \% \mathrm{SbCl}_{3}$ and $0.5 \% \mathrm{BiCl}_{3}$. $-\mathrm{Rhombs}$ greatly predominate. but "seeds" also abundant.

$0.015 \% \mathrm{SbCl}_{3}$ and $0.5 \% \mathrm{BiCl}_{3}$. $-\mathrm{Abundant}$ bismuth rhombs; no sign of antimony. The student should make up one or more of the above-described mixed solutions and test with $\mathrm{CsCl}$ in order to familiarize himself bimself with the appearance of the mixed crystals.

Double iodide test.-

Reggent: Solid potassium iodide (KI) and solid cesium iodide ( $\mathrm{CsCl})$.

Product: Antimony (or bismuth) cesium double iodide in orange-colored to red hexagons and stars.

Limit: $0.01 \% \mathrm{SbCl}_{3}$ solution; $0.005 \% \mathrm{BiCl}_{3}$ solution.

The mineral is broken by placing it in a drop of $1: 1 \mathrm{HNO}_{3}$ and evaporating to dryness. The residue is leached in a drop of $1: 5 \mathrm{HCl}$, and the drop is transferred to another place on the slide. 1 tiny fragment of potassium iodide is poked into one side of the drop. If antimony or bismuth chloride is present in 0.10 per cent or 
greater concentration, that part of the solution adjacent to the frag. ment is colored light yellow. Bismuth gives the solution a deeper yellow than antimony. If either element is absent or present in con. centrations weaker than 0.10 per cent of the chloride, no coloration of the solution takes place.

When the fragment of $\mathrm{KI}$ is dissolved, a fragment of $\mathrm{CsCl}$ is poked into the opposite side of the drop. Where the solutions come into contact the yellow color disappears and the double iodide of antimony or bismuth is precipitated in hexagons or stars. The antimony compound is orange-colored and the bismuth compound rose-red. (See pl. 10 and pl. 11, A, B.) Hexagons are most abundant, but stars are very common with antimony. Hexagons, some of them elongated, are the usual forms precipitated by bismuth. This forms a marked contrast to the corresponding double chloride of cesium and bismuth. Stars are seldom seen where bismuth is present to the exclusion of antimony.

If the solution is concentrated with respect to antimony, or if some compounds that interfere with the test are present, notably arsenic, tellurium, or $\mathrm{H}_{2} \mathrm{SO}_{4}$, the first antimony precipitate is likely to be in the form of irregular rounded grains. Usually some of these grains will act as nuclei around which well-formed hexagonal plates will later crystallize. The nuclei are of a darker color than the material added later. If the quantity of the antimony precipitate is great the color of the aggregate is distinctly reddish and is almost identical with that of the bismuth compound. If the antimony crystals themselves are thick they may be red and be mistaken for bismuth. This is the greatest objection to the double iodide test. In oblique reflected light the antimony crystals are likewise red. Beginners are apt to make mistakes in judging the color of the crystals. With experience this difficulty tends to be minimized, final determination on thick, more or less equidimensional crystals being avoided and thinner and more platy hexagons and stars sought. It is recommended that the student have on hand stock solutions of $\mathrm{SbCl}_{3}$ and $\mathrm{BiCl}_{3}$ in $1: 5 \mathrm{HCl}$ and that he occasionally check his ability to distinguish colors.

The bismuth crystals are more constant in character, and if suffcient iodide is present the bismuth hexagons are always red. A fer of the ore minerals contain both antimony and arsenic. The mineral is usually broken down with $1: 1 \mathrm{HNO}_{3}$ and then leached with three successive drops of 1: $7 \mathrm{HNO}_{3}$. Nearly all the arsenic is removed by this treatment, also nearly all the copper and other more basic minerals. Nevertheless it is very difficult to remove the final traces of arsenic.

Compounds containing both arsenic and bismuth are rare in nature or lacking altogether. 
The double iodide test was performed on solutions of $\mathrm{SbCl}_{3}$ in $1: 5$ $\mathrm{HCl}$ and $\mathrm{BiCl}_{3}$ in 1:5 HCl mixed in various proportions. The following results were obtained:

$0.5 \% \mathrm{SbCl}_{3}$ and $0.01 \% \mathrm{BiCl}_{3}$. - The usual orange-colored hexagons and stars characteristic of $\mathbf{S b}$; no sign of $\mathbf{B i}$.

$0.5 \% \mathrm{SbCl}_{3}$ and $0.02 \% \mathrm{BiCl}_{3}$. - Orange-colored hexagons predominate, but a few red hexagons are also present.

$0.5 \% \mathrm{SbCl}_{3}$ and $0.03 \% \mathrm{BiCl}_{8}$ - - Orange-colored stars and hexagons predominate, but some red stars and hexagons are also present. It is believed that red stars are indicative of the presence of both $\mathrm{Sb}$ and $\mathbf{B i}$.

$0.5 \% \mathrm{SbCl}_{3}$ and $0.06 \% \mathrm{BiCl}_{3}$. - Same as preceding. Orange-colored hexagons predominate over red.

$0.5 \% \mathrm{SbCl}_{3}$ and $0.12 \% \mathrm{BiCl}_{3}$. - Same as preceding, but red hexagons more numerous.

$0.5 \% \mathrm{SbCl}_{3}$ and $0.25 \% \mathrm{BiCl}_{3}$.-Red bismuth hexagons alone seen. No sign of $\mathrm{Sb}$.

As the proportion of bismuth in the solution increases the effect of the antimony on the characteristics of the mixed crystals decreases and finally becomes zero.

The following conclusions seem justified:

1. If either antimony or bismuth is present to the exclusion of the other, either the double iodide or double chloride test is satisfactory. However, the color effect of the double iodide would seem to make that test preferable.

2. If both antimony and bismuth are present, with bismuth predominant, only the double chloride test will indicate the presence of antimony.

3. If both antimony and bismuth are present, with antimony predominant, the double iodide test is preferable.

4. A small proportion of bismuth is detectable in the presence of a large proportion of antimony, but a small proportion of antimony is not detectable in the presence of a large proportion of bismuth.

5. If both elements are suspected, both the double chloride and double iodide tests should be performed.

Arsenic causes a precipitate identical in character with antimony cesium double iodide. Means for distinguishing between the arsenic and antimony compounds are discussed on page 159.

\section{Cesium chloride test.-}

TIN

Reagent: Solid cesium chloride.

Product: Cesium chlorostannate $\left(\mathrm{Cs}_{2} \mathrm{SnCl}_{6}\right)$, in colorless highly refracting octahedra.

Limit: $0.01 \% \mathrm{SnCl}_{2}$.

The test for tin is similar to the cesium chloride test for antimony. The mineral is broken down by $1: 1 \mathrm{HNO}_{3}$. The residue is leached $15122-31-12$ 
by three successive drops of $1: 7 \mathrm{HNO}_{3}$ to remove the soluble nitrates and finally with a drop of water to remove the remaining traces of $\mathrm{HNO}_{3}$. The tin remains in the residue. This is leached with a drop of 1:5 HCl, and the drop is transferred to another place on the slide and tested with a fragment of $\mathrm{CsCl}$ (method III). A precipitate of colorless highly refracting octahedra proves tin. (See pl. 11, D.)

Usually one drop of $1: 5 \mathrm{HCl}$ will not dissolve all of the tin in the residue. Three or four successive drops can be applied to the residue, each giving a good tin test.

Tin compounds behave similarly to those of antimony in that the tin does not dissolve in nitric acid but is oxidized to metastannic acid $\left(\mathrm{H}_{2} \mathrm{SnO}_{3}\right)$. This is a white amorphous powder which dissolves in $\mathrm{HCl}$, forming stannic chloride $\left(\mathrm{SnCl}_{4}\right)$. When a fragment of cesium (or rubidium) chloride is placed in a stannic chloride solution the insoluble compound cesium (or rubidium) chlorostannate is precipitated in colorless isometric crystals, which are almost invariably octahedra. The tin must be in the tetravalent condition in order to give this test. In testing the $\mathrm{SnCl}_{2}$. solution cited in the outline, a few drops of $\mathrm{H}_{2} \mathrm{O}_{2}$ were added to oxidize the tin to $\mathrm{SnCl}_{4}$.

If tin alone is to be tested for, the writer prefers rubidium to cesium chloride as a reagent. The rubidium chlorostannate octahedra are always larger and better formed than those of the corresponding cesium compound. The sensitivity of the rubidium chloride test seems to be as great as that of the cesium chloride test. ${ }^{88}$ The cesium salt is more insoluble than the rubidium salt and forms smaller crystals, frequently requiring a 4-millimeter objective for their discernment.

On the other hand, rubidium chloride is not a satisfactory reagent for identifying antimony and bismuth. Consequently in making a systematic series of tests on an unknown mineral it is better to use cesium chloride. If tin is present, a precipitate will form, and if this is unsatisfactory as far as the forms of the individual crystals are concerned, the test can be repeated with rubidium chloride.

The precipitate is sometimes so fine grained that the individual crystal forms can not be recognized. This indicates that the tin was not completely oxidized to the stannic condition. This is remedied by placing a drop of aqua regia on the original residue and evaporating to dryness. The residue is leached by a drop of $1: 5 \mathrm{HCl}$, and the drop is transferred to another place on the slide and tested with a fragment of rubidium chloride as before.

Antimony does not form an isomorphous compound with tin. When a mineral containing both antimony and tin is tested antimony forms the characteristic orange-colored hexagons of the double

88 The writer differs from Chamot in this regard. 
iodide, and tin forms colorless octahedra side by side with the antimony hexagons. (See detailed description of tests for franckeite and cylindrite, pp. 187, 188.)

The textbooks of Chamot and of Behrens and Kley state that if a fragment of potassium iodide is dissolved in the tin-bearing solution the cesium precipitate will be colored yellow. The present mriter is unable to agree with this statement. Under the conditions described here the octahedra remain colorless.

Lead forms a double salt with cesium similar to the tin salt. Nost of the lead is removed by leaching the original residue with 1:7 $\mathrm{HNO}_{3}$ and transferring the drop. By the addition of $\mathrm{HCl}$ the remaining lead is converted to $\mathrm{PbCl}_{2}$, which is almost insoluble in the $\mathrm{HCl}$. However, if lead is present in the mineral, it is best to add a fragment of $\mathrm{KI}$ to the $\mathrm{HCl}$ solution before adding the $\mathrm{CsCl}$. The double iodide of cesium and lead will form orange-colored needles, which will not interfere with the formation of the tin salt.

The tin test above described is highly satisfactory in every respect. The octahedra are sometimes almost spectacular in appearance. All the tin sulpho salts occurring in nature are readily broken down by $\mathrm{HNO}_{3}$. Cassiterite $\left(\mathrm{SnO}_{2}\right)$ is insoluble and must first be fused in a solium carbonate bead in order to be converted to a soluble form. The bead is then crushed and dissolved in a few drops of $1: 5 \mathrm{HCl}$ in a small capsule or annealing cup like that described by Chamot. ${ }^{80}$ 1 drop of the solution is drawn up into a capillary tube and blown ant on a glass slide. A fragment of $\mathrm{CsCl}$ is added to the drop. The presence of $\mathrm{NaCl}$ caused by dissolving in $\mathrm{HCl}$ the excess of $\mathrm{Na}_{2} \mathrm{CO}_{3}$ does not interfere with the formation of the tin salt. Of course, the amount of cassiterite required to give a satisfactory tin test is sereral times that of the sulpho salts.

\section{TELLURIUM}

\section{Cesium chloride test.-}

Reagent: Solid cesium chloride ( $\mathrm{CsCl})$.

Product: Cesium chlorotellurite $\left(\mathrm{Cs}_{2} \mathrm{TeCl}_{6}\right)$.

Limit: $0.01 \%$ Te in $1.5 \mathrm{HCl}$.

The mineral is broken down by $1: 1 \mathrm{HNO}_{3}$. The residue is leached with a drop of 1:5 HCl. The drop is then transferred and tested with a fragment of cesium chloride, by method III. Well-formed lemon-yellow isometric crystals are precipitated. If the concentration of tellurium is low the crystals begin to appear at the edge of the drop and the precipitation proceeds toward its center.

In concentrated solutions octahedra predominate in the precipilate. With lower concentrations pseudohexagonal plates and triingles are more common. These show no double refraction and are

"Chamot, E. M., op. cit., fig. 154, p. 294. 
probably isometric. (See pl. 9, A,B.). The composition of the crystals may be confirmed, as stated by Behrens and Kley, by adding a small fragment of KI to the center of the drop. If the amount added is small the crystals are stained brown, but if greater quan. tities are added the crystals are completely destroyed, and a precipitate of small amorphous brown grains is formed.

The principal difficulty with the tellurium test is that of getting the material into solution. A minimum of heat is required, otherwise anhydrous tellurious acid $\left(\mathrm{H}_{2} \mathrm{TeO}_{3}\right)$ forms instead of the normal compound $\mathrm{H}_{2} \mathrm{TeO}_{3} \cdot \mathrm{nH}_{2} \mathrm{O}$. The anhydrous variety is only sparingly soluble in even the strongest acids.

This test is exceedingly satisfactory in every respect. It is simple and reliable, and the product is highly characteristic.

The test does not work well in neutral or weakly acidic solutions. The strength of acid given above was determined by experiment, as follows: 0.5 gram of metallic tellurium was placed in a beaker containing 20 cubic centimeters of $1: 1 \mathrm{HNO}_{3}$, and the solution evaporated to dryness on a steam bath. The white powder, $\mathrm{H}_{2} \mathrm{TeO}_{3} \cdot \mathrm{nH}_{2} \mathrm{O}$ was taken into solution in 17 cubic centimeters of 1:1 HCl. When dissolved, 33 cubic centimeters of $\mathrm{H}_{2} \mathrm{O}$ was added to the solution, the result being 1 per cent of $\mathrm{Te}$ in $1: 5 \mathrm{HCl}$.

Potassium iodide test.-Potassium iodide added to a solution containing $\mathrm{H}_{2} \mathrm{TeO}_{3}$ precipitates a finely divided amorphous chocolatebrown powder consisting in part of the tetra-iodide of tellurium $\left(\mathrm{TeI}_{4}\right)$ and in part of the native element. When the $\mathrm{KI}$ is added the precipitate comes down immediately in the place where the fragment is added; then the precipitate spreads out toward the periphery of the drop in a widening ring. The precipitate in the center of the ring is redissolved, forming a liquid with a peculiar greenish tinge in transmitted light. This liquid is surrounded by the ring of brownish precipitate. The reaction is not easy to describe but is highly characteristic and when once observed will never be forgotten.

Selenious acid gives exactly the same reaction with KI. The precipitate is of a lighter shade of brown than the corresponding tellurium precipitate, and when the quantity precipitated is small the prevailing tint may be pinkish or orange-colored. The inexperienced observer, however, should make confirmatory tests on a fresh drop of test solution. (The solvent is, of course, 1:5 HCl.) First a fragment of $\mathrm{CsCl}$ is added to the drop. If a honey-yellow precipitate of isometric crystals forms, tellurium is present. If no precipitate forms, the drop is merged with a drop of stannous chloride solution (see p.167); a brick-red precipitate proves selenium.

All the amorphous precipitates described here are best observed in obliquely reflected light, with a 16-millimeter objective. 
The iodide test for tellurium is of particular value in that it is sometimes encountered in testing for other elements. It is the main reliance for identifying both selenium and tellurium in the systematic scheme of analysis described on pages 173-179.

\section{SELENIUIM}

\section{Stannous chloride test.-}

Reagent: $5 \% \mathrm{SnCl}_{2}$ solution in 1: $5 \mathrm{HCl}$.

Product: Native selenium, a brick-red powder.

Lmit: $0.05 \% \mathrm{SeO}_{2}$ in $\mathrm{H}_{2} \mathrm{O}$.

The red color of native selenium is very characteristic and when obtained no additional test is necessary to confirm the presence of the element. Most selenides are readily broken down by nitric acid. Usually metallic selenium is thrown down at once and can be observed best in oblique light. When once precipitated it can not be taken into solution with nitric acid or aqua regia. With some compounds, such as clausthalite, some metallic selenium is precipitated, but most of it goes into solution as selenious acid $\left(\mathrm{H}_{2} \mathrm{SeO}_{3}\right)$.

The usual procedure is as follows: Break down mine with 1:1 $\mathrm{HNO}_{3}$ and leach residue with a drop of $1: 5 \mathrm{HCl}$. A red tinge in the residue indicates selenium. To confirm it, transfer the filtrate and merge with it a drop of $5 \% \mathrm{SnCl}_{2}$ solution (method I). Selenium will be precipitated as a brick-red powder. This test is highly satisfactory, but the precipitate is amorphous, and it is best to use a larger amount of mineral than when testing for tellurium. An area on the mineral 0.35 millimeter in diameter is usually sufficient. Gold, mercury, and tellurium, if present in solution, will likewise be thrown down but rarely obscure the red color of the precipitate.

\section{Potassium iodide test.-}

\section{Reagent: Solid potassium iodide (KI).}

Product: Selenium iodide $\left(\mathrm{SeI}_{4}\right)$, a reddish-brown powder.

Limit: $0.02 \% \mathrm{SeO}_{2}$ in $\mathrm{H}_{2} \mathrm{O}$.

The mineral is broken down by repeated applications of $1: 1$ $\mathrm{HNO}_{3}$. Usually some of the selenium will be precipitated as the element, and the remainder will form the acid $\mathrm{H}_{2} \mathrm{SeO}_{3}$, which remains as a white powder when the solution is evaporated. The residue from evaporation is leached with a drop of 1:5 HCl, and the drop is transferred. A fragment of $\mathrm{KI}$ is placed in the center of the drop. A reddish-brown amorphous precipitate of $\mathrm{SeI}_{4}$ is thrown down immediately and spreads toward the periphery of the drop as 8 widening ring. (See description of the corresponding test for tellurium, p. 166.) The color is very similar to that of the corresponding Te precipitate but is a lighter shade of brown. 
Although the iodide reaction for selenium is more sensitive than the stannous chloride test, the color of the iodide precipitate is less characteristic than that of the native element. The iodide test is of value in that it is sometimes encountered in testing for other elements, notably antimony, bismuth, and tin.

Cesium chloride gives no precipitate with selenious acid, and this fact constitutes the best means of distinguishing between selenium and tellurium. When the iodide precipitate is obtained, it is best to take more material in solution with 1:5 HCl. Usually enough selenious or tellurious acid remains in the residue from the preceding test to yield another satisfactory test for the respective element. If not, fresh mineral powder is dissolved in 1:1 $\mathrm{HNO}_{3}$, and the residue is leached with a drop of 1:5 HCl. A fragment of cesium chloride is added. A precipitate of honey-yellow isometric crystals proves tellurium. If no precipitate forms, the solution contains selenium. This is confirmed by merging the drop with a drop of stannous chloride solution; a brick-red precipitate proves selenium.

\section{MANGANESE}

\section{Sodium bismuthate test.-}

Reagent: Solid sodium bismuthate $\left(\mathrm{NaBiO}_{3}\right)$.

Product: Sodium permanganate $\left(\mathrm{NaMnO}_{4}\right)$, which colors solution pink to purple.

Limit: $0.02 \% \mathrm{MnCl}_{2} .4 \mathrm{H}_{2} \mathrm{O}$ in $1: 7 \mathrm{HNO}_{3}$.

The oxides of manganese are only sparingly soluble in nitric acid but are readily soluble in $1: 1 \mathrm{HCl}$ and in aqua regia. The powder is taken into solution by means of three or four successive drops of either of these reagents. The residue, $\mathrm{MnCl}_{2} \cdot 4 \mathrm{H}_{2} \mathrm{O}$, collects as a ring around the periphery of the drop. It usually has a pinkish tinge and is distinctively crystalline, but the nature of the crystals can not be determined, owing to their small size.

The residue is taken into solution in a drop of $1: 7 \mathrm{HNO}_{3}$. A fragment of sodium bismuthate is added. This is a strong oxidizing agent and oxidizes the bivalent manganese to the permanganate state. The $\mathrm{MnO}_{4}$ ions color the solution pink to purple, the color depending on the concentration of $\mathrm{MnO}_{4}$. If the concentration is very low the color may not appear immediately but will be apparent near the edge of the drop when it begins to dry up.

The permanganate solution thus formed is not permanent under the conditions just described. In a short time, usually about a quarter of a minute, the permanganate ions break down and deposit manganese dioxide as a brown powder. The deposition of this powder begins at the edge of the drop and proceeds toward the center. The solution loses its color as the powder is deposited. 


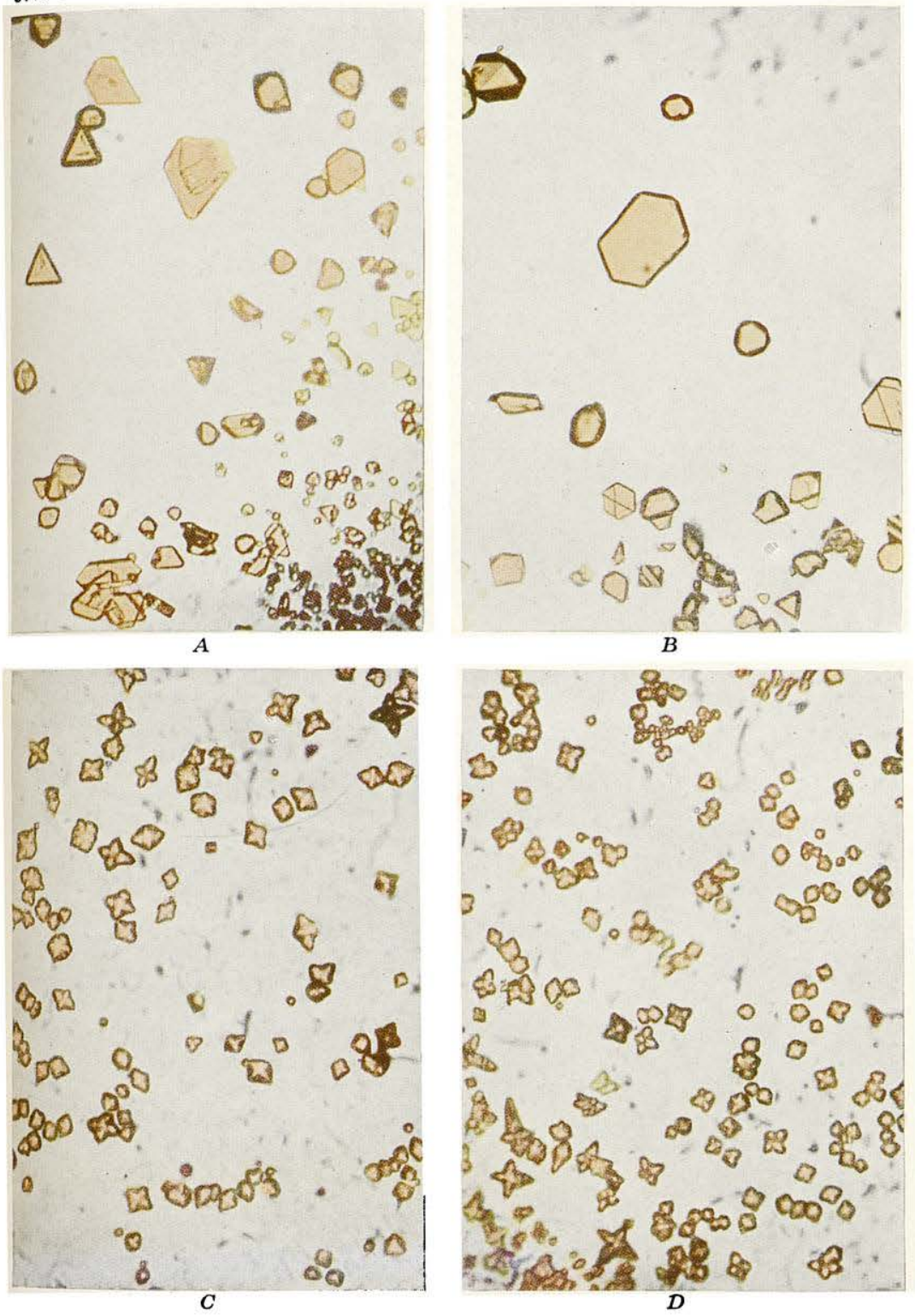

MICROCHEMICAL TESTS FOR ARSENIC AND TELLURIUM

A, Cesium chloro-tellurite crystals. Obtained from a $1: 5 \mathrm{HCl}$ solution containing $1 \%$ Te. $\times 70$. B. From same solution as $A$. $C$, Ammonium arseno-molybdate crystals. obtained from a $0.5 \%$ ammonium arsenate solution in $1: 7 \mathrm{HNO}_{3} \times 215$. $D$, From same solution as $C$. 

U. S. GEOLOGICAL SURVEY
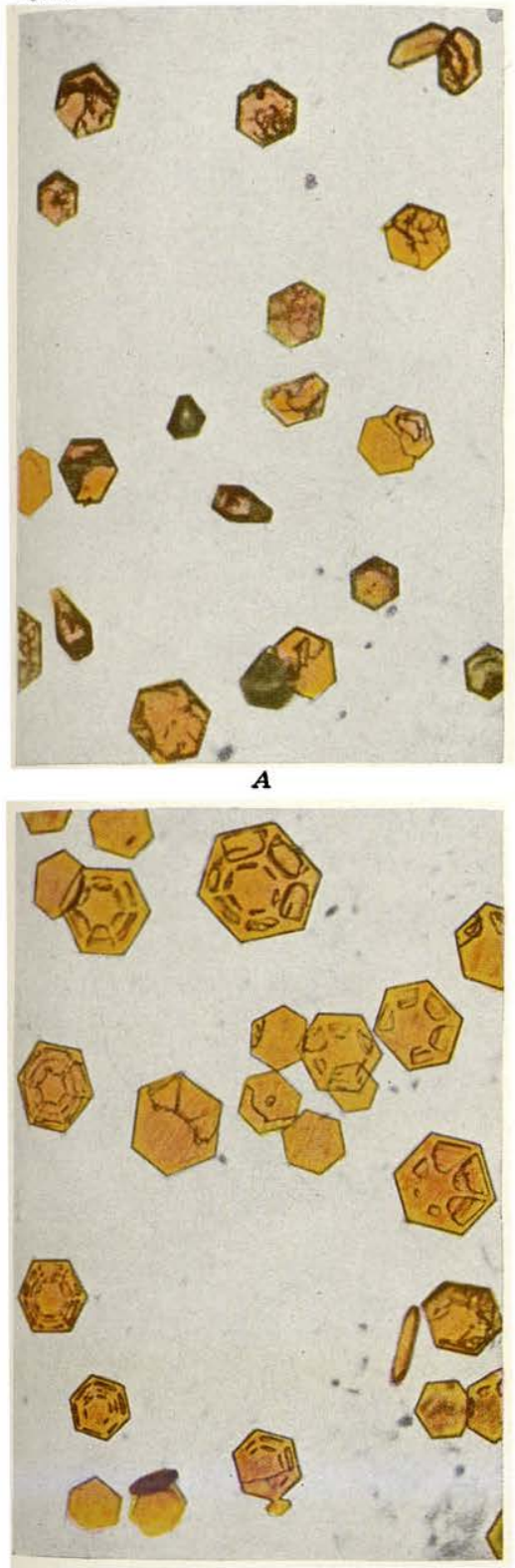

$\boldsymbol{C}$
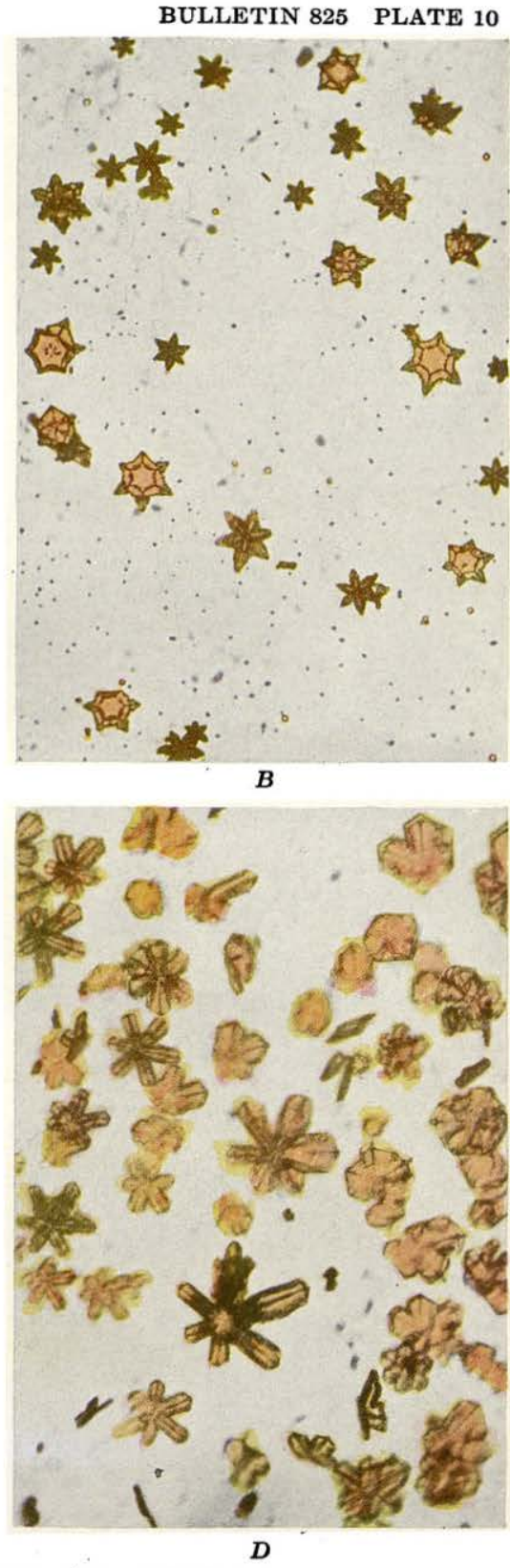

MICROCHEMICAL TESTS FOR ANTIMONY

$A, B, C, D$, Antimony-cesium iodide crystals obtained from a $0.5 \%$ antimony chloride solution in 1:5 $\mathrm{HCl}$. $\times 130$. 

U. S. GEOLOGICAL SURVEY
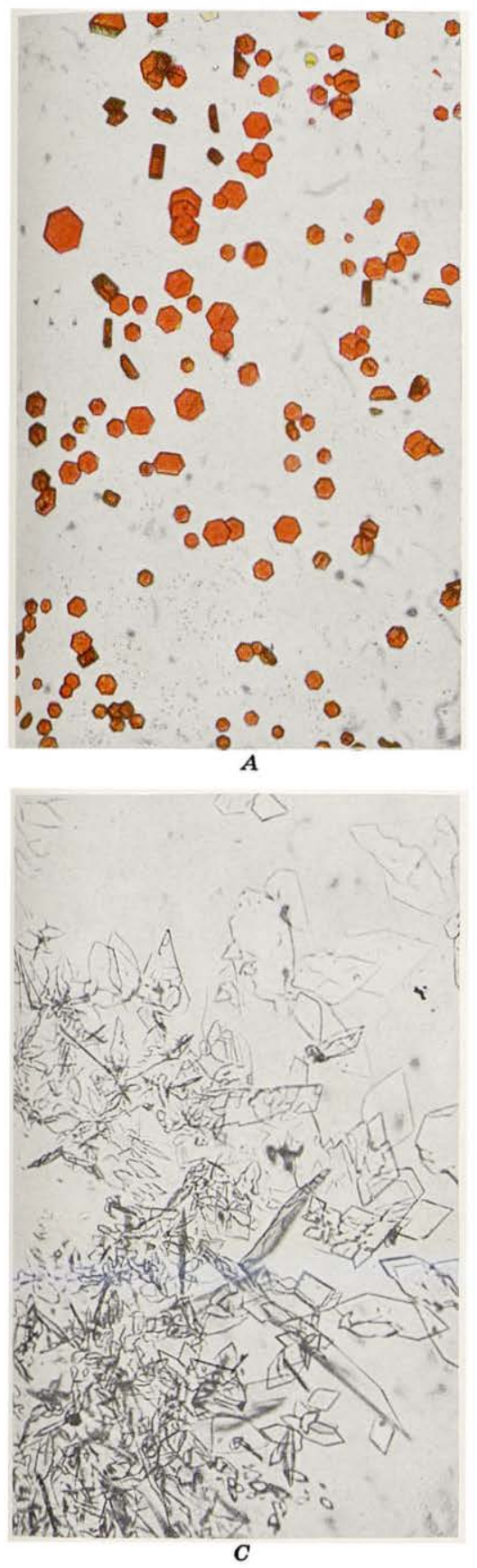

BULLETIN 825 PLATE 11
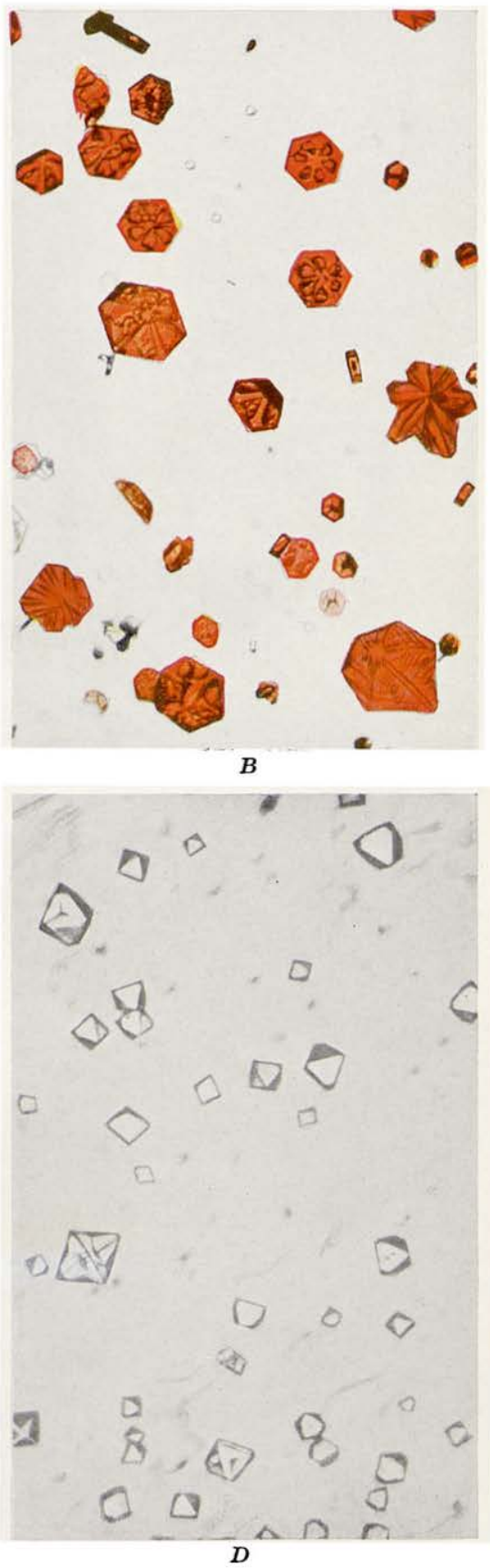

MICROCHEMICAL TESTS FOR BISMUTH AND TIN

$A, B$, Bismuth-cesium iodide crystals. $\times 250$. $C$, Bismuth-cesium chloride crystals. $\times 75 . \quad D$, Tin-rubidium chloride crystals. $\times 145$. 
It is advisable to use a fairly large quantity of sodium bismuthate, much more than is usual in method III. Only a part of the reagent is dissolved, the amount depending on the proportion of manganese present.

The sodium bismuthate test is described by Chamot but is not given the prominence which it merits. It is sensitive and highly distinctive, and other elements do not interfere with it. In addition it works in a strongly acid solution.

Some minerals, such as hübnerite $\left(\mathrm{MnWO}_{4}\right)$ and wolframite ( $\left.(\mathrm{Fe}, \mathrm{Mn}) \mathrm{WO}_{4}\right)$, can not be taken into solution by nitric or hydrochloric acids or by aqua regia. It is obvious that the sodium bismuthate test is not applicable to these minerals, and the sodium carbonate bead test used in blowpipe analysis is the best one to use.

\section{SULPHUR}

Reagent: Solid calcium acetate $\left(\mathrm{Ca}\left(\mathrm{C}_{2} \mathrm{H}_{3} \mathrm{O}_{2}\right)_{2}\right)$.

Product: Calcium sulphate (gypsum) crystals.

Limit: $0.15 \% \mathrm{H}_{2} \mathrm{SO}_{4}$.

Elemental sulphur and $\mathrm{H}_{2} \mathrm{~S}$.- - The element sulphur is known in several allotropic modifications, but the form existing in commercial deposits is the ordinary orthorhombic form, usually with crystal faces. It is soluble in carbon disulphide $\left(\mathrm{CS}_{2}\right)$, benzene $\left(\mathrm{C}_{6} \mathrm{H}_{6}\right)$, and a number of other organic solvents and can be recrystallized from these reagents, thus forming a quick and accurate test. Carbon disulphide is the most effective solvent, but it slowly decomposes, liberating elemental sulphur. If this reagent is employed, it should be tested by evaporating a drop on a glass slide. If a residue is observed the reagent should be rejected. Benzene forms a satisfactory solvent, but it evaporates with extreme rapidity. The powder to be tested is placed on a glass slide, and a cover glass about 3 millimeters in diameter is placed over it. A drop of benzene is brought to the edge of the cover glass by means of a platinum wire. When liberated from the wire, it immediately flows under the cover glass. Although partly protected by the cover glass the drop evaporates in about a minute, but meanwhile some sulphur has been dissolved, and as the drop evaporates the sulphur is precipitated in crystals. The crystals are large and tend to agglomrrate. Recognizable forms are not common, but straight edges are seen at the boundaries of the mass. The best indication of crystalline sulphur is the very high birefringence of the mass. The interference color is white of a high order, similar to that of carbonate crystals. In oblique reflected light crystalline sulphur is yellow.

Some simple sulphides, such as stibnite, sphalerite, and bismuthinite, and all or nearly all of the sulphosalts of bismuth yield a 
yellow globule of sulphur when decomposed by nitric acid. This is identical in appearance with ordinary fused sulphur but does not dissolve in carbon disulphide or benzene. However, the appearance of the globule itself is sufficiently indicative, and no other test for sulphur is necessary.

A few simple sulphides when treated with nitric acid evolve $\mathrm{H}_{2} \mathrm{~S}$ gas. The odor of this gas is very distinctive, and minute quantities are easily detectable. The test is carried out directly on the polished section, thus obviating the necessity of transferring powder to a glass slide. The minerals liberating $\mathrm{H}_{2} \mathrm{~S}$ are alabandite $(\mathrm{MnS})$, troilite $(\mathrm{FeS})$, and a small proportion of galena specimens.

By far the greater proportion of the sulphides and sulphosalts when decomposed by nitric acid deposit sulphur as a white amorphous powder. This powder is not sufficiently distinctive in appearance to constitute a test for sulphur. It is one of the allotropic forms of sulphur and like the yellow globule above described does not dissolve in carbon disulphide or benzene.

Sulphate test.-Attempts to take the amorphous white sulphur residue into solution on a glass slide have resulted in failure. The reagents tried were $1: 1 \mathrm{HNO}_{3}$, fuming $\mathrm{HNO}_{3}$, and aqua regia. It is possible to take this sulphur into solution by treatment in a beaker with fuming $\mathrm{HNO}_{3}$. The sulphur is oxidized to $\mathrm{H}_{2} \mathrm{SO}_{4}$ and in quantitative analysis is precipitated by barium chloride and weighed as barium sulphate. Oxidation of the sulphur in this manner requires continued boiling of the reagent and hence is not possible on a glass slide. A possible means of escaping this difficulty is to fuse the sulphide in a sodium carbonate or potassium nitrate bead. This requires much more material than has been used in tests already described, and high concentrations of sodium or potassium are introduced into the test drop. Hence this means is employed only as a last resort.

Fortunately in treating most sulphur-bearing minerals with $1: 1$ $\mathrm{HNO}_{3}$, some sulphur is oxidized to $\mathrm{H}_{2} \mathrm{SO}_{4}$ in addition to that deposited as the solid element. With some sulphides high in sulphur, such as pyrite, marcasite, and hauerite $\left(\mathrm{MnS}_{2}\right)$, a relatively large proportion of the sulphur is oxidized. The same is true of minerals readily soluble in $\mathrm{HNO}_{3}$, such as chalcocite and sphalerite. Sulphides difficultly soluble in $\mathrm{HNO}_{3}$ and many sulpho salts yield a relatively lower proportion of $\mathrm{H}_{2} \mathrm{SO}_{4}$. In evaporating a mineral to dryness care should be taken not to raise the temperature so high that the $\mathrm{H}_{2} \mathrm{SO}_{4}$ begins to evaporate. Fortunately $\mathrm{H}_{2} \mathrm{SO}_{4}$ is not very volatile, and to evaporate it requires a higher temperature and more time than is usually employed in making microchemical tests. With reasonable care very little is lost by evaporation. 
The common elements yielding insoluble sulphates are barium, strontium, calcium, and lead. The most insoluble sulphate and theoretically the one which should yield the most sensitive test is barium. However, under the conditions here described the precipitate is always amorphous and can be recrystallized only in strong sulphuric acid. Strontium acetate forms with sulphite ions an amorphous precipitate in neutral solutions and weak acids. With high magnifications a few tiny orthorhombic crystals can usually be observed at the edge of the amorphous mass. In strong $\mathrm{HCl}$ strontium sulphate is more soluble, and more of the precipitate is crystalline. This precipitate should also be observed with high magnification. The crystals are colorless and highly refracting but too small to show birefringence with crossed nicols. The most common forms are orthorhombic bipyramids resembling octahedra, blunt prisms with rounded terminations, and oval spherulites. Like barium sulphate the strontium compound can be recrystallized in hot, concentrated $\mathrm{H}_{2} \mathrm{SO}_{4}$, but this is, of course, prohibited in testing for sulphates. It is useful in testing for strontium with $\mathrm{H}_{2} \mathrm{SO}_{4}$. The crystals so obtained are larger, more uniform in character, and hence more distinctive than those obtained from strong $\mathrm{HCl}$. The strontium reaction can be used as a test for the presence of sulphate ions, but the corresponding calcium test is better, for it yields more distinctive crystals. The limit of dilution with the strontium reaction is about 0.05 per cent of $\mathrm{H}_{2} \mathrm{SO}_{4}$, or three times that of the calcium test.

Lead acetate furnishes a much more satisfactory test for sulphate ions than the strontium salt. Lead sulphate has a stronger crystallizing tendency than strontium sulphate, and a larger percontage of the precipitate is crystalline, although most of it is amorphous. The most characteristic forms of lead sulphate are tiny colorless diamond-shaped or boat-shaped plates and slender prisms with sharp-pointed terminations. Many of these prisms have enlargements or protuberances, oval or diamond-shaped in cross section, at the center of the prisms. The limit of possible dilution with this test is about 0.02 per cent of $\mathrm{H}_{2} \mathrm{SO}_{4}$. The reagent is used in solid form and is applied to the center of the drop. The lead sulphate test would be very satisfactory were it not for the fact that other ions, notably those of arsenic acid, also precipitate lead. Lead arsenate resembles lead sulphate, and if arsenic is present in a substance, sulphur can not be tested for in this manner.

Calcium acetate precipitates sulphates from a neutral or weak hydrochloric acid solution. The reaction is less satisfactory in a nitric acid solution. Calcium sulphate is more soluble than the sulphates of strontium and lead, and the tendency to crystallize is strong, hence the precipitate is entirely crystalline. From neutral 
or weak acid solutions the product is gypsum $\left(\mathrm{CaSO}_{4} \cdot 2 \mathrm{H}_{2} \mathrm{O}\right)$; from strong acid solutions anhydrite $\left(\mathrm{CaSO}_{4}\right)$ predominates. Under the conditions here described gypsum alone will form. Gypsum ordinarily is not precipitated immediately from solutions low in $\mathrm{SO}_{4}$ ions. The first crystals are usually precipitated near the edge of the drop, and precipitation proceeds toward the center. The crystals are colorless and very characteristic. Long, slender needles predominate. The terminations show various forms. Usually they are very irregular, and many resemble the frayed ends of wood splinters. In somewhat more concentrated solutions the ends may show regular crystal faces inclined at an angle to the prism, indicating that the crystal form is monoclinic. A highly characteristic form is a reentrant angle in either or both ends, giving a fishtail or swallowtail appearance to the crystals. Arrowhead twins, although occasionally encountered, are not common. The birefringence is low, and the crystals are so thin that polarization colors are usually not observed. The prisms are often so slender that they resemble fibers. These tend to interlock and form a mat.

Arsenic acid precipitates calcium as a white amorphous calcium arsenate powder from neutral solutions. Calcium arsenate is kept in solution by a 5 per cent or stronger $\mathrm{HCl}$ solution. The best gypsum crystals are obtained with as weak an acid solution as can be used. Consequently, the 5 per cent $\mathrm{HCl}$ solution is recommended for the sulphate test.

Aqua regia is the most effective reagent for oxidizing sulphur. The mineral is broken down by two or more applications of aqua regia. The residue is cooled and then leached with a drop of 5 per cent $\mathrm{HCl}$. This drop is transferred to another place on the slide, and a fragment of calcium acetate is added at its center. The characteristic gypsum crystals should appear in half a minute or less. The amount of material required to give a good sulphate test is about double that used with most tests. An area on the polished section about 0.35 millimeter in diameter is sufficient.

Arsenic acid and ferric chloride both delay the crystallization of gypsum but do not prevent it entirely. Arsenic acid is a gelatinous substance and if present in relatively concentrated proportion prevents the diffusion of the elements through the solution. Ferric chloride has practically the same effect. Consequently if too much material containing arsenic and iron is used in too small a drop, the resulting sulphur test will be unsatisfactory. If the calcium acetate dissolves very slowly or fails to dissolve, it indicates that either or both of these detrimental compounds are present. The student should practice with arsenopyrite until he can get a good sulphate test at every attempt. The following modification of the procedure above described is suggested: 
Break down the mineral with two successive drops of aqua regia, evaporating each to dryness. Leach the residue with a drop of 5 per cent $\mathrm{HCl}$, transfer the drop, and add a fragment of calcium acetate. If the fragment dissolves slowly or fails to dissolve, the concentration of arsenic acid or ferric chloride or both is probably too high. It is then necessary to wait until the drop is nearly dry before gypsum crystals begin to form, and they may not form at all. If the expected gypsum crystals fail to appear add a drop of aqua regia to the original residue, evaporate to dryness, leach with 5 per cent $\mathrm{HCl}$, and test for sulphates with calcium acetates as before. The reason for this modification is that arsenic is much more readily oxidized by aqua regia than sulphur. The first attack on the mineral will oxidize most of the arsenic and take into solution most of the iron. When these are removed in the first drop of 5 per cent $\mathrm{HCl}$, the proportion of arsenic acid and iron with respect to sulphur in the residue is much lower. After the second attack the effect of the detrimental compounds is much less, and the sulphate test can be carried out successfully.

In conclusion, the writer does not advocate that the sulphur test be made except where absolutely necessary. The great majority of ore minerals contain sulphur, and identification of the metallic elements usually suffices to determine the mineral. The sulphate test is necessary, however, to distinguish between such minerals as arsenopyrite ( $\mathrm{FeAsS})$ and löllingite $\left(\mathrm{FeAs}_{2}\right)$ or between chloanthite $\left(\mathrm{NiAs}_{2}\right)$ and gersdorffite (NiAsS). Earthy minerals containing sulphur, such as hauerite $\left(\mathrm{MnS}_{2}\right)$, should be tested for sulphur in this manner.

\section{A SYSTEMATIC SCHEME OF MICROCHEMICAL ANALYSIS 90}

Microchemical tests are applicable to any mineral provided it can be taken into solution. However, it is usually most convenient to determine transparent minerals by optical methods. The best of these methods consists in determination of the indices of refraction of the mineral by immersing the powdered mineral in liquids of known indices of refraction.

The scheme of analysis described here is almost entirely confined to the opaque ore minerals. The opaque minerals are easily identified under the reflecting microscope by their color in vertically reflected light. This is discussed more fully on pages 30 and 34 .

As already stated (p. 2), it is best to identify the opaque ore mineral by means of hardness, polarization, and etching tests and then confirm the identification by microchemical tests for one or

This scheme is based on one originated by the writer's former students, Drs. H. J. Brdiser and H. A. Powers. 
more elements. However, this scheme may fall down because of errors in the etch tables, mistakes in interpreting polarization or etching effects, or errors in the formulas of the minerals as given in the standard texts, or because the minerals may be "new "- that is, not yet described in the literature. Any one of these factors would cause a failure in the scheme. The next resource will then be to identify the elements of the mineral by a systematic scheme similar to that of qualitative chemical analysis.

Any systematic scheme of this kind is greatly simplified by making the hardness test at the outset. This is described on page 35 but is outlined here for convenience. The mineral is tested for hardness by means of a sharp needle. Minerals that scratch readily are classified as soft, and those that scratch with difficulty or not at all are classified as hard. The advantage of making this classification at the outset is that certain elements are rarely if ever found in the hard group and others are rarely if ever found in the soft group.

\section{HARD MINERALS}

The hard opaque minerals may be divided into three groups, as follows:

A. Insoluble or very difficultly soluble in concentrated $\mathrm{HNO}_{3}$, 1:1 $\mathrm{HNO}_{3}$, or aqua regia. This group consists mostly of the ironbearing oxide minerals, also cassiterite, tungstates, and rare-earth minerals.

B. Readily soluble in 1:1 $\mathrm{HNO}_{3}$ or aqua regia. This group consists mostly of the nickel-cobalt-iron arsenides, antimonides, sulpharsenides, and sulphantimonides; also pyrite and marcasite.

C. Insoluble or slightly soluble in $\mathrm{HNO}_{3}$ but readily soluble in aqua regia and 1:1 $\mathrm{HCl}$. This group consists of the hard manganese oxides.

Group A.-The No. 9 needle ordinarily used will not scratch a hard mineral. A powder may usually be obtained by using a No. 6 or stronger needle, the point of which is ground back by means of the emery wheel so as to give a relatively large cutting edge. By working it back and forth in a pit on the surface of the mineral, powder will gather in front of the needle. A nonmetallic red powder indicates hematite; brick-red to light brown indicates the hydrous iron oxides of the "limonite" group, also chromite; dark brown indicates franklinite or braunite; a black metallic powder is probably magnetite. All these minerals except braunite are almost insoluble in strong nitric acid and aqua regia. However, by many repeated applications of aqua regia, each evaporated over the microflame, enough material can be taken into solution to give an iron test. The residue is colored orange to brown. It is taken into 
solution with a drop of 1: $7 \mathrm{HNO}_{3}$ and tested for iron with a fragment of $\mathrm{K}_{4} \mathrm{Fe}(\mathrm{CN})_{6}$.

If the mineral is too hard to yield any powder by the method just described it is probably a rare-earth mineral. As already stated, these minerals are not considered in this investigation.

Group B.-The minerals of group B are readily broken down by means of $1: 1 \mathrm{HNO}_{3}$ or aqua regia. Minerals of the nickel-cobaltarsenic-antimony group tend to be associated together. Niccolite is recognized at sight from its color. Its neighbors can be assumed to belong to that group until proved otherwise. This group is large, and nearly all the minerals in it look almost exactly alike. In reflected light they are ashy gray and "hard" (in the sense already mentioned). With experience one can always tell from color and hardness that a mineral with the above description is chloanthite, löllingite, gersdorffite, skutterudite, rammelsbergite, smaltite, kallilite, willyamite, hauchecornite, safflorite, or ullmanite. Arsenopyrite and löllingite can not be told apart by etching, polarization, or microchemical tests. They are slightly different in color, however, arsenopyrite being galena-white and löllingite ashy gray. Arsenopyrite tends to be associated with pyrrhotite, sphalerite, galena, and chalcopyrite; and löllingite with the other arsenides in the group just described.

A hard white mineral soluble in nitric acid will contain only nickel, cobalt, iron, arsenic, antimony, bismuth, or sulphur. Dissolve the mineral in either $\mathrm{HNO}_{3}$ or aqua regia. Notice the residue : A white amorphous powder indicates sulphur or antimony.

A gelatinous ring around the drop indicates arsenic.

If the residue is green look for nickel; if yellow or orange, iron; if pink, cobalt.

Tests for all the elements of this group can be made from the above-mentioned residue. Leach the residue with 1 per cent $\mathrm{HNO}_{3}$.

1. Iron, nickel, cobalt, and arsenic go into the solution. Transfer 1 per cent $\mathrm{HNO}_{3}$ drop and evaporate almost to dryness. Add drop of ammonium molybdate solution and heat very slowly till dry. Add drop of 1:7 $\mathrm{HNO}_{3}$, which dissolves the excess of ammonium molybdate, leaving minute yellow octahedra or twinned isometric crystals if arsenic is present. Filter the solution from these crystals, transfer to another place on the slide, and add potassium mercuric thiocyanate (method I). If iron is present, the solution turns red. If cobalt is present, it forms acicular blue crystals, which usually are grouped as crosses or rosettes. If nickel is present, minute brown spherulites form slowly near the edge of the drop.

2. Antimony, bismuth, and sulphur remain undissolved in the original residue. The formation of a flocculent precipitate at this stage 
indicates bismuth. Leach original residue with at least three suc. cessive drops of 1 per cent $\mathrm{HNO}_{3}$ to remove remaining traces of arsenic, then a drop of $\mathrm{H}_{2} \mathrm{O}$ to remove the nitric acid. Then leach original residue with a drop of 1:5 HCl. Transfer drop and add fragment of KI. If an amorphous yellow or orange-colored precipitate forms, some arsenic is still present. If solution turns light yellow, it contains antimony or bismuth, or both. Add fragment of $\mathrm{CsCl}$. Orange-colored hexagons prove antimony; red hexagons prove bismuth.

There are only three hard minerals that contain antimonykallilite $(\mathrm{Ni}(\mathrm{Bi}, \mathrm{Sb}) \mathrm{S})$, ullmanite $(\mathrm{Ni}(\mathrm{As}, \mathrm{Sb}) \mathrm{S})$, and willyamite $((\mathrm{Co}, \mathrm{Ni}) \mathrm{SbS})$.

Group C.-If a hard mineral is almost insoluble in $1: 1 \mathrm{HNO}_{3}$ but readily soluble in aqua regia, the presence of manganese should be tested for by leaching the residue with a drop of $1: 7 \mathrm{HNO}_{3}$, transferring the drop, and adding solid sodium bismuthate. If the drop turns pink or purple, manganese is present.

\section{SOFT MINERALS}

The soft minerals are divided into three groups-(A) soluble in 1:1 $\mathrm{HNO}_{3}$, (B) insoluble in 1:1 $\mathrm{HNO}_{3}$ but soluble in aqua regia, (C) insoluble in both $1: 1 \mathrm{HNO}_{3}$ and aqua regia. Group $\mathrm{C}$ is not considered in the analytical scheme here proposed.

Group A.-A soft mineral soluble in nitric acid will not contain mercury. Examine the residue for following hints:

A white amorphous powder indicates sulphur or antimony.

A gelatinous ring indicates either arsenic or silver.

A curdy white structureless mass suggests bismuth or tellurium.

A white lattice or boxlike structure indicates lead; more rarely an excess of silver may give a similar structure.

Sulphur sometimes leaves a light-yellow agglutinated mass instead of a white powder.

An agglutinated brick-red mass proves selenium.

If the residue is green, suspect copper or nickel; if yellow or orange-colored, iron.

Selenium occurs only in combination with silver, gold, bismuth, copper, mercury, lead, tellurium, and sulphur.

Step 1, for iron, copper, zinc, cobalt, nickel, and silver: Leach the residue from the original mineral solution with a drop of 1 per cent $\mathrm{HNO}_{3}$. Transfer the drop, and merge with it a drop of potassium. inercuric thiocyanate. If iron is present, the test solution will turn red; copper forms a crystalline precipitate consisting of greenishvellow mosslike clumps, prisms, and crosses; zinc forms white or gray feathery crosses; silver forms an amorphous white powder; 
cobalt forms indigo-blue prisms; nickel forms small brown spherulites near the edge of the drop.

Step 2, for copper, arsenic, selenium, and tellurium: Leach the original residue with $\bullet$ a drop of $\mathrm{H}_{2} \mathrm{O}$ to remove traces of $\mathrm{HNO}_{3}$. Remove the drop. Then leach the original residue with a drop of $1: 5$ HCl. Any silver remaining from the preceding test will come down here as curdy white chloride. Lead compounds are changed to the chloride, which often appears as white needles. Transfer the filtrate to another place on slide. Add a fragment of KI. Any copper remaining from the preceding test will come down as an amorphous white, gray, or light-yellow powder; arsenic will come down as an amorphous yellow to orange-colored powder; tellurium or selenium will come down as a fine-grained amorphous chocolate-brown powder; if antimony or bismuth is present in appreciable amount ( 0.1 per cent or more of $\mathrm{SbCl}_{3}$ ), the solution will be colored yellow in the neighborhood of the KI fragment. The selenium and tellurium precipitates come down instantly. The arsenic and copper precipitates are sometimes slow in forming. Therefore, it is best to observe the drop until it has nearly evaporated, then add sufficient $1: 5 \mathrm{HCl}$ to give the drop its original volume. Once formed, the copper and arsenic precipitates are not soluble in the additional acid. All the precipitates thrown down by KI are amorphous and are best observed in oblique reflected light. The tellurium and selenium precipitates will not be confused with the arsenic and copper precipitates, owing to the peculiar manner in which the tellurium and selenium reactions proceed. (See detailed description, p. 167.) When a tellurium or selenium precipitate is obtained, confirmatory tests by the cesium chloride and stannous chloride reactions should be made in letermine whether the precipitate is tellurium or selenium. The confirmatory tests can usually be made by leaching the original residue with another drop of $1: 5 \mathrm{HCl}$ and testing the resulting solution. If not enough material remains in the original residue to yield these tests, it may be necessary to make them on additional mineral powder. The arsenic and copper precipitates are very much alike-a fact which may cause some confusion in identification. The arsenic precipitate is yellower than the copper precipitate and sometimes is distinctly orange-colored. If there is any doubt as to the identity of the precipitate, it is advisable to make the ammonium molybdate test for arsenic on fresh mineral powder. It should be remembered that a large part, possibly most of the arsenic, has been removed from the original residue by leaching the residue with 1 per cent $\mathrm{HNO}_{3}$ and the subsequent drop of water and removal of both drops. This arsenic is lost in making the thiocyanate test for other elements (step 1). Hence, failure to get the iodide test for arsenic at this stage does not indicate that no arsenic was present in the mineral. 
'This is the weakest point in the entire analytical scheme. However, in nine cases out of ten there. is enough arsenic acid remaining to give a good indication for arsenic when tested with KI. As already indicated, if there is any reason to believe that arsenic is present in the mineral and the iodide test fails to show it, the molybdate test for arsenic should be made on additional powdered mineral.

Step 3, for arsenic, antimony, bismuth, and tin: A fragment of cesium chloride is added to the same drop in which the KI test has just been made. The drop should first be restored to its original size by adding more 1:5 HCl. If arsenic is present, part of the iodide precipitate formed by the preceding test is converted to the cesium arsenic double iodide, which forms as an orange-colored amorphous precipitate near the border of the drop. Usually the concentration in arsenic is not great enough to cause the formation of the orange-colored hexagons described on page 158. The precipitate is best observed in oblique reflected light. Antimony will form orangecolored hexagons; bismuth will form red hexagons; tin will form colorless octahedra.

Step 4, for lead, gold, sulphur, and selenium: Examine the original residue in obliquely reflected light, using a 16-millimeter objective. Gold, if present, will be seen as yellow metallic grains; any selenium not converted to the soluble acid and removed in the preceding operation remains behind in the original residue as the native element, which is observed as a reddish powder or agglutinated mass. When $\mathrm{HCl}$ was added in step 2, the lead was converted into the chloride $\left(\mathrm{PbCl}_{2}\right)$. Usually the lead chloride needles can be recognized at sight under the microscope. It is always advisable, however, to make the iodide test for lead, whether the chloride crystals are recognizable or not. Volatilize the $\mathrm{HCl}$ by adding a drop of water to the residue and evaporating to dryness. Cool and add a drop of water to the residue. Transfer the drop to another place on the slide and add a fragment of KI. Lead, if present, will form lemon-yellow hexagons and disk-shaped plates of the normal iodide. To confirm the presence of gold, add a drop of aqua regia to the residue, evaporate to dryness over the microflame. Gold is converted to an orangecolored to brown residue that is very soluble in water. Leach this residue with a drop of water, transfer the drop, and merge with it a drop of pyridine- $\mathrm{HBr}$ solution. Pleochroic red crystals prove gold.

The following observations on the soft minerals attacked by $\mathrm{HNO}_{3}$ are of value: The presence of arsenic excludes bismuth, tellurium, mercury, selenium, and zinc; the presence of nickel excludes copper, gold, lead, mercury, selenium, silver, and zinc.

Group B.-A soft mineral insoluble in $\mathrm{HNO}_{3}$ but easily soluble in aqua regia probably contains mercury or manganese. If $\mathrm{Hg}$ is 
present, the $\mathrm{HgCl}_{2}$ residue will usually show a lattice pattern. (See pl. 5, D.) A manganese residue forms as a circular ring with a faint tinge of pink.

Test for mercury: Dissolve in aqua regia. Leach the residue with 1 per cent nitric acid. Transfer the drop, and add a small fragment of $\mathrm{Co}\left(\mathrm{NO}_{3}\right)_{2}$. When dissolved, add a fragment of KCNS. Blue branching or acicular crystals, the same as seen in the test for cobalt, prove the presence of mercury. Mercury is associated only with antimony, selenium, tellurium, and sulphur.

Test for manganese: Dissolve in aqua regia. Leach the residue with a drop of 1: $7 \mathrm{HNO}_{3}$, transfer the drop, and add solid sodium bismuthate. If the solution turns pink or purple, manganese is present.

\section{SPECIFIC TESTS FOR MINERALS}

The systematic scheme of microchemical analysis described above is designed to identify in as few operations as possible all the principal elements occurring in ore minerals. The tests given in the scheme are those which are applicable to the greatest number of elements. The test outlined for any particular element is not necessarily the best test for that element. For instance, the tests with potassium mercuric thiocyanate for both silver and nickel are given because that reagent will indicate the presence of a considerable number of additional elements; but the ammonium bichromate test for silver and the dimethyl glyoxime test for nickel are more characteristic for the respective elements than the thiocyanate tests.

As a rule, the investigator, by means of polarization, color, hardness, and etch tests, has a definite clue to the identity of the mineral under investigation. He then desires to confirm his observation by tests for the elements he believes are contained in the mineral. In the following pages the procedure believed to be the best for identifying the elements in each mineral is described in detail. In using this procedure many short cuts are possible, in comparison with the systematic scheme. For instance, there is no particular need of making a thiocyanate test on galena. It is hoped that the use of these short cuts will result in much saving of time.

: Aguilarite $\left(\mathrm{Ag}_{2}(\mathrm{Se}, \mathrm{S})\right)$.-Decompose mineral with 1:1 $\mathrm{HNO}_{3}$. Usually several applications of acid are necessary. The residue is gelatinous and may be tinged yellow and reddish. Leach residue with drop of 1 per cent $\mathrm{HNO}_{3}$, transfer drop, and add fragment of ammonium bichromate. The presence of selenious acid will delay but not prevent the formation of silver bichromate. Red crystals prove silver. Leach original residue with drop of 1:5 HCl. Any silver remaining is converted into white flocculent chloride. Transfer filtrate and merge with it a drop of stannous chloride. A brick-red amorphous precipitate proves selenium. If desired the silver chloride in the residue can be tested by the ammonia method. Remove all traces of selenium by several

$15122-31-13$ 
successive drops of 1:5 $\mathrm{HCl}$; remove traces of $\mathrm{HCl}$ with a drop of water. Then place a drop of concentrated $\mathrm{NH}_{4} \mathrm{OH}$ on residue. After a few seconds colorless highly refracting isometric crystals will separate out of the solution. (See description of naumannite, p. 195.)

Aikinite $\left(\mathrm{Cu}_{2} \mathrm{~S} .2 \mathrm{PbS} . \mathrm{Bi}_{2} \mathrm{~S}_{3}\right)$. -Decompose mineral with one or two applications of $1: 1 \mathrm{HNO}_{3}$. Aikinite is very easily attacked by $\mathrm{HNO}_{2}$. The residue is white and curdy. Carefully examine residue for lattice structure (lead nitrate). Leach residue with drop of 1 per cent $\mathrm{HNO}_{3}$, transfer drop, and evaporate to dryness. To the residue from the evaporation add a drop of 1 per cent acetic acid; add fragment of sodium acetate and potassium nitrite to the drop. If black cubes of the triple nitrite do not form at once, add fragment of cesium chloride. Black and brown cubes prove lead and copper. Some of the bismuth was carried orer in the nitric acid solution; most remains in the original residue. Leach this with a drop of 1:5 HCl. Transfer drop and add fragment of KI. The solution near the fragment is colored yellow ( $\mathrm{Sb}$ or $\mathrm{Bi}$ ). Then add fragment of $\mathrm{CsCl}$. Red hexagons prove bismuth.

Alabandite $(\mathrm{MnS})$.-Take mineral into solution with $1: 1 \mathrm{HNO}_{3}$. Evapo. rate, cool, and leach residue with a drop of 1: $7 \mathrm{HNO}_{3}$. Add fragment of sodium bismuthate. Solutions turn pink or purple, proving manganese.

Alaskaite $\left(\mathrm{Ag}_{2} \mathrm{~S} . \mathrm{PbS} .2 \mathrm{Bi}_{2} \mathrm{~S}_{3}\right)$.-Decompose mineral with 1:1 $\mathrm{HNO}_{3}$. Leach residue with drop of 1 per cent $\mathrm{HNO}_{3}$. Transfer drop and add fragment of ammonium bichromate. A yellow amorphous precipitate indicates lead, and red crystals indicate silver. The two precipitates do not mask each other.

The original residue contains bismuth and most of the lead. Add drop of 1: $5 \mathrm{HCl}$, transfer drop; and add fragment of KI. Solution turns yellow near fragment. Then add fragment of $\mathrm{CsCl}$. Red hexagons prove bismuth. Leach original residue with three more drops of $1: 5 \mathrm{HCl}$ to remove last traces of bismuth. Then add three successive drops of water to original residue. Transfer each drop to another place on slide and add fragment of KI. The first drop may not give a satisfactory test for lead, owing to the presence of remaining traces of $\mathrm{HCl}$ and $\mathrm{Bi}$. The second or third drop will yield yellow hexagons, which prove lead.

Algodonite $\left(\mathrm{Cu}_{0} \mathrm{As}\right)$.-Take mineral into solution with 1:1 $\mathrm{HNO}_{3}$. Residue is gelatinous with tinge of green. Leach residue with drop of 1:7 $\mathrm{HNO}_{3}$, transfer drop, and heat slowly by passing in and out of flame. When nearly dry add directly a drop of ammonium molybdate solution. Again heat slowly over flame till dry. Add drop of 1:7 $\mathrm{HNO}_{3}$ solution to redissolve excess of ammonium molybdate and examine. Tiny yellow isometric crystals prove arsenic. Transfer filtrate (containing copper nitrate and dissolved ammonium molybdate) and merge with it a drop of potassium mercuric thiocyanate solntion. A yellowish-green precipitate proves copper.

Altaite $(\mathrm{PbTe})$.-Decompose mineral with 1:1 $\mathrm{HNO}_{3}$. Examine residue for lattice structure. Leach residue with drop of 1:5 HCl. Transfer drop and add fragment of $\mathrm{CsCl}$. Honey-yellow isometric crystals prove tellurium. Add to original residue a drop of water and evaporate drop over flame to remove $\mathrm{HCl}$. Cool and again add drop of water to original residue and transfer drop. Add to it a fragment of KI. Yellow. hexagons prove lead.

Andorite ( $\left.\mathrm{Ag}_{2} \mathrm{~S} .2 \mathrm{PbS} .3 \mathrm{Sb}_{2} \mathrm{~S}_{3}\right)$.-Decompose mineral with 1:1 $\mathrm{HNO}_{3}$. The mineral is somewhat resistant to attack, but with a liberal supply of finely divided material and at least 10 successive drops of liquid, enough can be taken into solution to yield the tests. Leach residue with a drop of $1: 5 \mathrm{HCl}$, transfer drop, and test for $\mathrm{Sb}$ by meas's of $\mathrm{KI}$ and $\mathrm{CsCl}$. Orange-colored hexagons prove antimony. Leach original residue with two additional drops of $\mathrm{HCl}$ to 
remove remaining traces of $\mathrm{Sb}$ and reject drops. Then leach original residue with three successive drops of $\mathrm{H}_{2} \mathrm{O}$ and add to each a small fragment of $\mathrm{KI}$. Yellow glistening hexagons prove lead. Finally leach original residue with drop of concentrated $\mathrm{NH}_{4} \mathrm{OH}$. Observe edge of drop with a 4-millimeter objective. Tiny, highly refracting isometric crystals of AgCl separate from solution after a few moments. The bichromate test for silver has been successfully used on andorite, but it requires about three times the amount of material ordinarily used for microchemical tests and, in addition, more than an arerage degree of technical skill.

Antimony (Sb).-Decompose mineral with 1:1 $\mathrm{HNO}_{3}$. Residue is white and powdery. Leach residue with drop of 1:5 HCl, transfer drop, and add fragment of KI. Solution turns yellow near fragment. Add fragment of CsCl. orange-colored hexagons and stars prove antimony.

Aramayoite $\left(\mathrm{Ag}_{2} \mathrm{~S}\right.$. $\left.(\mathrm{Sb}, \mathrm{Bi})_{2} \mathrm{~S}_{3}\right)$.-Decompose mineral with 1:1 $\mathrm{HNO}_{3}$. Leach residue with drop of 1 per cent $\mathrm{HNO}_{3}$, transfer drop, and add fragment of ammonium bichromate. Red crystals prove silver. Leach original residue with drop of 1:5 HCl. Transfer drop and add fragment of KI. Solution turns yellow near fragment. Add fragment of $\mathrm{CsCl}$. Both orange-colored and red hexagons form, proving antimony and bismuth.

Argentite $\left(\mathrm{Ag}_{2} \mathrm{~S}\right)$.-Decompose mineral with 1:1 $\mathrm{HNO}_{3}$. Leach residue with 1 per cent $\mathrm{HNO}_{3}$, transfer drop, and add fragment of ammonium bichromate. Red crystals prove silver.

Argyrodite $\left(4 \mathrm{Ag}_{2} \mathrm{~S} . \mathrm{GeS}_{2}\right)$.-Argyrodite is very resistant to attack by $1: 1 \mathrm{HNO}_{2}$, but by repeated applications (usually about ten suffice) enough material can be taken into solution to give the silver test. Leach residue with drop of 1 per cent $\mathrm{HNO}_{3}$, transfer drop, and add fragment of ammonium bichromate. Red crystals prove silver. No good test for germanium is available.

Arsenargentite ( $\left.\mathrm{Ag}_{3} \mathrm{As}\right)$.-Take mineral into solution with $1: 1 \mathrm{HNO}_{3}$. Leach residue with drop of 1 per cent $\mathrm{HNO}_{3}$, transfer drop, and add fragment of ammonium bichromate. Red crystals prove silver. Take more mineral into solution with 1:1 $\mathrm{HNO}_{3}$. Add drop of 1:5 HCl to residue to prec pitate the silver: Evaporate to dryness, cool, and add drop of 1:7 $\mathrm{HNO}_{3}$ to residue. Transfer drop and slowly evaporate over microflame. When nearly dry add drop of ammonium molybdate solution and again evaporate slowly over microflame. When dry, cool and add drop of 1:7 $\mathrm{HNO}_{3}$ to residue. Tiny yellow isometric crystals prove arsenic.

Arsenic (As).-Mineral goes completely into solution in 1:1 $\mathrm{HNO}_{3}$, leaving no residue. Evaporate almost to dryness. Leach residue with drop of ammonium molybdate reagent and evaporate slowly to dryness over flame. Cool and leach with a drop of 1:7. $\mathrm{HNO}_{3}$. Tiny yellow isometric erystals prove arsenic.

Arsenoferrite $\left(\mathrm{FeAs}_{2}\right)$.-Test identical with that for arsenopyrite.

Arsenopyrite (FeAsS).-Decompose mineral with 1:1 $\mathrm{HNO}_{3}$. The residue usually has a tinge of yellow or orange. Leach residue with drop of 1:7 $\mathrm{HNO}_{3}$, transfer drop, and evaporate slowly by pass ng slide in and out of microflame. When nearly dry add directly a drop of ammonium molybdate solution and again heat slowly over microflame. When dry, cool and add drop of $1: 7 \mathrm{HNO}_{3}$ to redissolve excess ammonium molybdate. Examine under microscope. Tiny yellow isometric crystals prove arsenic. Take up filtrate and transfer to another place on slide. Merge with it a drop of potassium mercuric thiocyanate solution. A red coloration of test solution proves iron.

Baumhauerite (4PbS.3As $\mathrm{S}_{3}$ ).-Decompose mineral with 1:1 $\mathrm{HNO}_{3}$. Leach residue with drop of $1: 5 \mathrm{HCl}$, transfer drop, and evaporate almost to dry- 
ness. Leach residue from the evaporation with drop of ammonium molybdate solution. Evaporate slowly to dryness. Cool and add drop of 1:7 $\mathrm{HNO}_{2}$. Tiny yellow isometric crystals prove arsenic. Leach original residue with three successive drops of $\mathrm{H}_{2} \mathrm{O}$, transfer drops, and add to each a fragment of KI. Yellow glistening hexagonal plates prove lead.

Benjaminite ( $\left.(\mathrm{Cu}, \mathrm{Ag})_{2} \mathrm{~S} .2 \mathrm{PbS} .2 \mathrm{Bi}_{2} \mathrm{~S}_{3}\right)$. -Decompose mineral with $1: 1 \mathrm{HNO}$. Leach residue with drop of 1 per cent $\mathrm{HNO}_{3}$, transfer drop, and merge with it a drop of potassium mercuric thiocyanate solution. Greenish-yellow mosslike clumps and prisms prove copper, and an amorphous white powder proves silver. Leach original residue with drop of 1:5 $\mathrm{HCl}$, transfer drop, and add fragments of $\mathrm{KI}$ and $\mathrm{CsCl}$. Red hexagonal plates prove bismuth. Leach original residue with two successive drops of $1: 5 \mathrm{HCl}$ to remove most of the remaining bismuth; reject both drops, then leach original residue with three successive drops of $\mathrm{H}_{2} \mathrm{O}$. Add to each a fragment of $\mathrm{KI}$. The first drop may not give a satisfactory test, owing to presence of remaining traces of $\mathrm{HCl}$. This keeps a trace of bismuth in solution, which tends to modify the lead iodide precipitate. The second or third drop will give good yellow hexagons, which prove lead.

Berthierite (FeS. $\mathrm{Sb}_{2} \mathrm{~S}_{3}$ ).-Decompose mineral with 1:1 $\mathrm{HNO}_{3}$. Leach residue with drop of 1:7 $\mathrm{HNO}_{3}$, transfer drop, and add fragment of $\mathrm{K}_{4} \mathrm{Fe}(\mathrm{CN})_{\text {e. }}$ A blue amorphous precipitate proves iron. Leach original residue with drop of 1: $5 \mathrm{HCl}$, transfer drop, and add fragment of KI. Solution is colored yellow near fragment. Then add fragment of cesium chloride. Yellow hexagons and stars prove antimony.

Berthonite $\left(2\left(\mathrm{~Pb}, \mathrm{Cu}_{2}\right) \mathrm{S}_{2} \mathrm{Sb}_{2} \mathrm{~S}_{3}\right)$. -Decompose mineral with $1: 1 \mathrm{HNO}_{3}$. Leach residue with drop of $1: 7 \mathrm{HNO}_{3}$, transfer drop, and evaporate to dryness. To residue from this evaporation add drop of 1 per cent acetic acid. Add successive fragments of sodium acetate and potassium nitrite to the acetic acid solution. If black and brown cubes do not come down at once add fragment of $\mathrm{CsCl}$. The formation of the cubes proves the presence of both lead and copper. Leach original residue with two additional drops of $\mathrm{HNO}_{3}$ to remove all the copper before testing for Sb. Both drops are rejected. Dry original residue and add to it a drop of 1:5 HCl. Transfer drop and add fragment of KI. Solution is colored yellow near fragment. Then add fragment of $\mathrm{CsCl}$. Orange-colored hexagons and stars prove antimony.

Bismuth (Bi).-Dissolve mineral in 1:1 $\mathrm{HNO}_{3}$. All goes into solution. Evaporation leaves white curdy residue. Take this into solution with 1:5 HCl. Add fragment of KI. Solution turns vivid yellow. Then add fragment of CsCl. Red hexagons prove bismuth.

Bismuthinite $\left(\mathrm{Bi}_{2} \mathrm{~S}_{3}\right)$.-Decompose mineral with 1:1 $\mathrm{HNO}_{3}$. Add drop of 1: $5 \mathrm{HCl}$ to residue. The bismuth goes into solution as chloride; the sulphur remains as an agglutinated yellow mass. Transfer filtrate, then add successive fragments of $\mathrm{KI}$ and $\mathrm{CsCl}$. Red hexagons prove bismuth.

Bismutoplagionite $\left(5 \mathrm{PbS} .4 \mathrm{Bi}_{2} \mathrm{~S}_{3}\right)$.-Decompose mineral with $1: 1 \mathrm{HNO}_{3}$. Leach residue with drop of 1:5 HCl, transfer drop, and add to it fragments of $\mathrm{KI}$ and $\mathrm{CsCl}$. Red hexagonal plates prove Bi. Leach original residue with two successive drops of $1: 5 \mathrm{HCl}$ to remove most of the remaining bismuth; reject both drops. Then leach original residue with three successive drops of $\mathrm{H}_{2} \mathrm{O}$. Add to each a fragment of $\mathrm{KI}$. The first drop may not give a satisfactory lead test, owing to remaining traces of $\mathrm{HCl}$. This keeps a trace of bismuth in solution, which tends to modify the lead iodide precipitate. The second or third drop will give good yellow hexagons, proving lead. 
Bormite $\left(\mathrm{Cu}_{6} \mathrm{FeS}_{4}\right)$.-Decompose mineral with 1:1 $\mathrm{HNO}_{3}$. Leach residue with 1:7 $\mathrm{HNO}_{3}$. Transfer drop and merge with it a drop of potassium mercuric thiocyanate solution (method I). Part of the drop turns pink, indicating iron. $\mathbf{A}$ greenish-yellow crystalline precipitate indicates copper. The iron test may be faint unless a relatively large amount of mineral is used. A microchemical test for bornite is rarely needed.

Boulangerite (5PbS.2Sb $\left.{ }_{2} \mathrm{~S}_{3}\right)$.-Decompose mineral with $1: 1 \mathrm{HNO}_{3}$. Examine residue for lattice structure, indicating lead. Leach residue with drop of 1:5 HCl. Transfer drop and add fragment of KI. Solution turns yellow near fragment as it dissolves. Then add fragment of cesium chloride. Orangecolored hexagons and stars prove antimony. Add drop of water to original residue and evaporate over flame to remove remaining traces of $\mathrm{HCl}$. Cool and again add drop of water to original residue. Transfer drop and add fragment of KI. Glistening yellow hexagons prove lead.

Bournonite $\left(\mathrm{Cu}_{2} \mathrm{~S} .2 \mathrm{PbS} . \mathrm{Sb}_{2} \mathrm{~S}_{3}\right)$. -Decompose mineral with $1: 1 \mathrm{HNO}_{3}$. Examine residue for lead nitrate lattice. Leach residue with a drop of 1:7 $\mathrm{HNO}_{3}$. Transfer drop and evaporate to dryness. To the residue from this evaporation add drop of 1 per cent acetic acid. Then add successive fragments of sodium acetate and potassium nitrite to drop. If brown and black cubes of the triple nitrite de not form at once, add a fragment of CsCl. Cubic crystals of the triple nitrite prove lead and copper. Leach original residue with two additional drops of $\mathrm{HNO}_{3}$, followed by one of water to remove all the copper before testing for antimony. Reject all three drops. Add to original residue a drop of 1:5 HCl. Transfer drop and add fragment of KI. Solution is colored yellow near the fragment as it dissolves. Add fragment of $\mathrm{CsCl}$. Orangecolored hexagons and stars prove antimony.

Braunite $\left(3 \mathrm{Mn}_{2} \mathrm{O}_{3} \cdot \mathrm{MnSiO}_{2}\right)$.-Decompose mineral with several successive applications of aqua regia. Leach residue with drop of $1: 7 \mathrm{HNO}_{3}$, transfer drop, and add solid sodium bismuthate. Solution turns purple near fragments, proving manganese.

Bravoite $\left((\mathrm{Fe}, \mathrm{Ni}) \mathrm{S}_{2}\right)$.-Decompose mineral with $1: 1 \mathrm{HNO}_{3}$. Leach residue with 1:7 $\mathrm{HNO}_{s}$, transfer drop, and evaporate to dryness. To residue from this evaporation add directly a drop of 1 per cent $\mathrm{NH}_{4} \mathrm{OH}$. A gelatinous yellow or orange-colored precipitate proves iron. Allow drop to evaporate slowly in the air. When evaporation is almost complete but the residue is still moist add drop of dimethyl glyoxime solution (method III-B, p. 125). A pink precipitate proves nickel.

Broithauptite (NiSb).-Decompose mineral with 1:1 $\mathrm{HNO}_{3}$. Leach residue with 1: $7 \mathrm{HNO}_{3}$, transfer drop, and evaporate to dryness. Add drop of dimethyl glyoxime solution directly to the residue from this evaporation. A pink precipitate proves nickel. Leach original residue with a drop of 1:5 $\mathrm{HCl}$, transfer drop, and add fragment of KI. Solution turns yellow near fragment as it dissolves. Then add fragment of $\mathrm{CsCl}$. Orange-colored hexagons and stars prove antimony.

Brongniardite $\left(\mathrm{Ag}_{2} \mathrm{~S} . \mathrm{PbS} . \mathrm{Sb}_{2} \mathrm{~S}_{3}\right)$. - Decompose mineral with 1:1 $\mathrm{HNO}_{3}$. Leach , residue with drop of 1 per cent $\mathrm{HNO}_{3}$. Transfer drop and add a fragment of ammonium bichromate. Red crystals, usually needlelike, prove silver, and an amorphous yellow precipitate proves lead. Leach original residue with a drop of 1:5 HCl. Transfer drop and add fragment of KI. Solution turns yellow near fragment. Then add fragment of $\mathrm{CsCl}$ to opposite side of drop. Orangecolored hexagonal plates prove antimony. If necessary lead can be confirmed by adding a drop of $\mathrm{H}_{2} \mathrm{O}$ to the original residue, transferring drop, and adding a fragment of KI. Yellow glistening hexagonal plates prove lead. 
Calaverite ( $\left.(\mathrm{Au}, \mathrm{Ag}) \mathrm{Te}_{2}\right)$.-Decompose mineral with $1: 1 \cdot \mathrm{HNO}_{3}$. One or two applications of acid suffice. The residue is a white curdy powder. Leach residue with 1:5 HCl. The white curdy residue goes into solution at once. Transfer drop and add a fragment of CsCl. Honey-yellow isometric crystals prove tellurium.

Gold remains in the original residue in small metallic grains and lumps. Usually examination in oblique reflected light, with a 16-millimeter objective, suffices to identify it. If the appearance of the residue is not sufficiently characteristic, add drop of aqua regia to residue and evaporate to dryness. An orange-colored residue of gold chloride remains. Dissolve this in water, transfer drop, and merge with it a drop of pyridine-HBr reagent. Strongly pleochroic (colorless to deep red) prisms prove gold.

Silver remains in the original residue as the curdy white chloride. Leach it with two or three successive drops of $1: 5 \mathrm{HCl}$ to remove remaining traces of gold and tellurium, then one of water to remove $\mathrm{HCl}$. Then add to original residue a drop of concentrated $\mathrm{NH}_{4} \mathrm{OH}$. Observe with an 8-millimeter objective. in a few seconds tiny colorless isometric crystals of $\mathrm{AgCl}$ will be seen floating in the solution.

Canfieldite (4 $\mathrm{Ag}_{2} \mathrm{~S} . \mathrm{SnS}_{2}$, with some germanium).-Decompose mineral with several applications of aqua regia. Leach residue with drop of 1:5 HCl, transfer drop, and add fragment of rubidium chloride. Highly refracting colorless octahedra prove tin. Germanium is not tested for. Add drop of water to original residue and evaporate to dryness to remove last traces of HCl. Then add to original residue a drop of concentrated $\mathrm{NH}_{4} \mathrm{OH}$. Observe drop with 8-millimeter objective. After a few seconds tiny colorless, highly refracting isometric crystals of $\mathrm{AgCl}$ will be seen floating in the solution.

Carrollite $\left((\mathrm{Co}, \mathrm{Cu}) \mathrm{S}^{-} \mathrm{Co}_{2} \mathrm{~S}_{3}\right)$.-Decompose mineral with $1: 1 \mathrm{HNO}_{3}$. Leach residue with drop of 1:7 $\mathrm{HNO}_{3}$, transfer drop, and merge with it a drop of potassium mercuric thiocyanate solution. Copper precipitates as yellowishgreen prisms and mosslike clumps, and cobalt precipitates separately as indigoblue prisms.

Cassiterite $\left(\mathrm{SnO}_{2}\right)$.-Fuse mineral in sodium carbonate bead in loop of platinum wire. Dissolve residue in a small capsule containing a few drops of 1:5 HCl. Pick up a drop by means of capillary tube and transfer to slide. Add to the drop a fragment of rubidium chloride. Highly refracting colorless octahedra prove tin.

Cerargyrite $(\mathrm{AgCl})$.-Place fragment of mineral in a drop of concentrated $\mathrm{NH}, \mathrm{OH}$. In a few seconds crystals of $\mathrm{AgCl}$ will separate out of the solution. Frequently these are skeletal and opaque. They are white in obliquely reflected light. The prevalent forms are right-angle crosses and stars in which three arms $120^{\circ}$ apart radiate from a center. A careful search and repeated drops of ammonia will almost always yield well-formed transparent cubes and other isometric forms.

Chalcocite $\left(\mathrm{Cu}_{2} \mathrm{~S}\right)$.-Decompose mineral by means of $1: 1 \mathrm{HNO}_{3}$. One application suffices. Leach residue with drop of 1:7 $\mathrm{HNO}_{3}$, transfer drop, and merge with it a drop of potassium mercuric thiocyanate solution. Greenish-yellow mosslike clumps and needlelike prisms prove copper.

Chalcophanite $\left((\mathrm{Mn}, \mathrm{Zn}) 0.2 \mathrm{MnO}_{2} \cdot 2 \mathrm{H}_{2} \mathrm{O}\right)$. - Take mineral into solution with aqua regia. Leach residue with $1: 7 \mathrm{HNO}_{3}$. Transfer drop and merge with a drop of potassium mercuric thiocyanate. A precipitate of violet or purple prisms proves zinc. Some crosses are usually to be seen, but the greater majority of the crystals are arrow-shaped or short prisms with ragged ends. Take fresh material into solution with aqua regia, leach residue with drop 
of 1: $7 \mathrm{HNO}_{3}$, transfer drop, and add solid sodium bismuthate fragments. Solution turns purple near fragments, proving manganese.

Chalcopyrite $\left(\mathrm{CuFeS}_{2}\right)$.-Decompose mineral by several applications of 1:1 $\mathrm{HNO}_{3}$. Residue is orange-colored or yellow. Leach residue with drop of 1:7 $\mathrm{HNO}_{3}$, transfer drop, and merge with it a drop of potassium mercuric thiocyanate. Greenish-yellow mosslike clumps and needlelike prisms prove copper. The test drop turns reddish, proving iron.

Chalcostibite $\left(\mathrm{Cu}_{2} \mathrm{~S}_{2} \mathrm{Sb}_{2} \mathrm{~S}_{3}\right)$.-Decompose mineral by means of 1:1 $\mathrm{HNO}_{3}$. Leach residue with drop of $1: 7 \mathrm{HNO}_{3}$, transfer drop, and merge with it a drop of potassium mercuric thiocyanate solution. Greenish-yellow mosslike clumps and prisms prove copper. Leach residue with two successive drops of 1:7 $\mathrm{HNO}_{3}$ to remove copper; reject both drops. Then leach residue with a drop of 1:5 $\mathrm{HCl}$, transfer drop, and add fragment of $\mathrm{CsCl}$. The solution near the fragment turns yellow as the KI dissolves. Then add fragment of $\mathrm{CsCl}$. Orange-colored hexagons and stars prove antimony.

Chloanthite $\left(\mathrm{NiAs}_{2}\right)$.-Take mineral into solution with aqua regia. Leach residue with drop of 1:7 $\mathrm{HNO}_{3}$, transfer drop, and slowly evaporate till nearly dry. Then add drop of ammonium molybdate solution and again slowly evaporate by passing the slide over the microflame several times. When dry, cool and add drop of 1:7 $\mathrm{HNO}_{3}$ to redissolve the excess of ammonium molybdate. Examine with 8-millimeter objective. Tiny yellow isometric crystals prove arsenic. Transfer filtrate to another place on slide and merge with it a drop of potassium mercuric thiocyanate solution. Small brown spherulites prove nickel. If desired this test may be confirmed by drying drop and adding dimethyl glyoxime solution directly to the spherulites. A pink precipitate will form if nickel is present. The reason the thiocyanate test is given precedence here is that iron and cobalt are usually contained isomorphously in chloanthite, and these are immediately precipitated by the thiocyanate solution.

Chromite $\left(\mathrm{FeO} \cdot \mathrm{Cr}_{2} \mathrm{O}_{3}\right)$. - The powder is very resistant to acids, but enough of it can be taken into solution for an iron test by placing powder in aqua regia and evaporating to dryness. Usually about 10 successive drops will suffice. Leach residue with drop of 1:7 $\mathrm{HNO}_{3}$, transfer drop, and add fragment of $\mathrm{K} \mathrm{Fe}(\mathrm{CN})_{\text {. }}$. A blue amorphous precipitate proves iron. There is no satisfactory test for chromium.

Cinnabar (HgS).-Decompose mineral by means of aqua regia. Examine residue for mercuric chloride lattice structure. Leach residue with drop of 1 per cent $\mathrm{HNO}_{3}$, transfer drop, and add fragment of $\mathrm{Co}\left(\mathrm{NO}_{3}\right)_{2}$. When dissolved add fragment of potassium thiocyanate. Indigo-blue prisms and branching forms of cobalt mercuric thiocyanate prove mercury.

Clausthalite ( $\mathrm{PbSe})$.-Decompose mineral by meaus of 1:1 $\mathrm{HNO}_{3}$. Examine residue for lead nitrate lattice structure and a red tinge, which indicates selenium. Leach residue with drop of 1:5 HCl, transfer drop, and merge with it a drop of stannnous chloride solution. A brick-red amorphous precipitate proves selenium. Add a drop of water to original residue and evaporate over microflame to remove traces of $\mathrm{HCl}$. Cool, then add another drop of water to original residue. Transfer drop and add to it a fragment of KI. Glistening yellow hexagons prove lead.

Cobaltite (CoAsS).-Decompose mineral by means of $1: 1 \mathrm{HNO}_{3}$. Leach residue with drop of 1:7 $\mathrm{HNO}_{3}$, transfer drop, and slowly evaporate by placing slide in and out of microflame several times. When nearly but not entirely dry add drop of ammonium molybdate solution and again slowly evaporate over flame. When dry, cool and add drop of 1:7 $\mathrm{HNO}_{3}$ to redissolve excess of ammonium molybdate. Examine microscopically with 8-millimeter objective. 
Tiny yellow isometric crystals prove arsenic. Transfer filtrate to another place on slide and merge with it a drop of potassium mercuric thiocyanate solution (method I). Indigo-blue prisms prove cobalt. If the solution turns pinkish, iron is also present.

Coloradoite (HgTe).-Decompose mineral by means of aqua regia. Leach residue with drop of 1 per cent $\mathrm{HNO}_{3}$, transfer drop, and add to it a fragment of cobalt nitrate. When dissolved, add a fragment of potassium thiocyanate (KCNS) to the solution. Indigo-blue branching forms and prisms prove mercury. Leach original residue with drop of 1:5 HCl. Transfer drop and add fragment of $\mathrm{CsCl}$. Honey-yellow isometric crystals prove tellurium.

Copper $(\mathrm{Cu})$.-Dissolve mineral in $1: 1 \mathrm{HNO}_{3}$. One application is sufficient. Leach residue with drop of 1:7 $\mathrm{HNO}_{3}$, transfer drop, and merge with it a drop of potassium mercuric thiocyanate solution. Greenish-yellow mosslike clumps and prisms prove copper.

Oorynite (NiAsS, with $\mathrm{Sb}$; antimonial gersdorffite).-Decompose mineral with 1:1 $\mathrm{HNO}_{3}$. Leach residue with 1:7 $\mathrm{HNO}_{3}$, transfer drop, and evaporate slowly. When nearly dry add drop of ammon um molybdate solution and again evaporate slowly till completely dry. Add drop of 1:7 $\mathrm{HNO}_{3}$ to residue in order to redissolve excess of ammonium molybdate. Tiny yellow isometric crystals prove arsenic. Transfer filtrate and merge with it a drop of potassium mercuric thiocyanate. Small brown spherulites prove nickel. Leach original residue with two successive drops of $1: 7 \mathrm{HNO}_{3}$ and one of $\mathrm{H}_{2} \mathrm{O}$ in order to remove remaining traces of arsenic. Then leach residue with drop of 1: $5 \mathrm{HCl}$, transfer drop, and add fragment of $\mathrm{CsCl}$. Colorless hexagons prove antimony.

Cosalite $\left(2 \mathrm{PbS} \mathrm{Bi}_{2} \mathrm{~S}_{3}\right)$.-Decompose mineral with 1:1 $\mathrm{HNO}_{3}$. Examine residue for lead nitrate lattice. Leach residue with 1:5 HCl. Transfer drop and add fragment of KI. Solution turns yellow near fragment as it dissolves. Then add fragment of $\mathrm{CsCl}$. The yellow color disappears near fragment and red hexagons form, proving bismuth. Leach original residue with two successive drops of 1:5 HCl, rejecting both. This will remove most of the remaining trace of bismuth. Then add three successive drops of water to or ginal residue. Transfer each drop to another place on slide and test with fragment of KI. The first drop may not give a satisfactory test for lead, owing to the presence of remaining traces of lead and bismuth, but the second or third drop will yield yellow hexagonal plates, which prove lead.

Covellite (CuS).-Decompose mineral with 1:1 $\mathrm{HNO}_{3}$. Leach residue with drop of 1:7 $\mathrm{HNO}_{3}$, transfer drop, and merge with it a drop of potassium mercuric thiocyanate solution. Greenish-yellow mosslike clumps and prisms prove copper.

Cubanite (CuS.2FeS).-Decompose mineral by several applications of 1:1 HNOs. Residue is orange-colored or yellow. Leach residue with drop of 1:7 $\mathrm{HNO}_{3}$, transfer drop; and merge with it a drop of potassium mercuric thiocyanate. Greenish-yellow mosslike clumps and prisms prove copper. A reddish color in the test drop proves iron.

Cuprite $\left(\mathrm{Cu}_{2} \mathrm{O}\right)$.-Dissolve mineral with 1:1 $\mathrm{HNO}_{3}$. Leach residue with drop of 1:7 $\mathrm{HNO}_{3}$, transfer drop, and merge with it a drop of potassium mercuric thiocyanate solution. Greenish-yellow mosslike clumps and prisms prove copper.

Cuprodescloizite $\left(\mathrm{Pb}_{2}(\mathrm{Zn}, \mathrm{Cu})_{2} \mathrm{~V}_{2} \mathrm{O}_{2} \cdot \mathrm{H}_{2} \mathrm{O}\right)$.-Dissolve mineral with $1: 1 \mathrm{HNO}_{3}$. Leach residue with drop of 1 per cent $\mathrm{HNO}_{3}$, transfer drop, and merge with it a drop of potassium mercuric thiocyanate solution. Greenish-yellow mosslike clumps and prisms prove copper. If zinc is present, the typical zinc precipitate 
should appear. (See description of zinc-copper isomorphous precipitate on p. 130.) Two specimens of cuprodescloizite from different localities tested by the writer gave negative zinc tests. Leach original residue with drop of 1:5 $\mathrm{HCl}$, remove drop from residue, and reject it. Add drop of water to original residue and evaporate to dryness over microflame to volatilize remaining trace of $\mathrm{HCl}$, cool, leach original residue with drop of water, transfer drop, and add fragment of KI. Yellow hexagons prove lead. Vanadium not testerl for by writer.

Cylindrite $\left(6 \mathrm{PbS} . \mathrm{Sb}_{2} \mathrm{~S}_{3} \cdot 6 \mathrm{SnS}_{2}\right)$.-Decompose mineral with 1:1 $\mathrm{HNO}_{3}$. Leach residue with 1:5 HCl, transfer drop, and add fragment of KI. Solution turns yellow near fragment as it dissolves. Add fragment of $\mathrm{CsCl}$ to solution. The yellow color of the solution disappears, and orange-colored hexagons and colorless octahedra form, proving antimony and tin. Leach original residue with drop of water and evaporate over microflame in order to volatilize remainder of $\mathrm{HCl}$. Cool, leach original res due with drop of water, transfer drop, and aid fragment of $\mathrm{KI}$. Yellow hexagons prove lead.

Delafossite $\left(\mathrm{CuFeO}_{2}\right)$. - Dissolve mineral in 1:1 $\mathrm{HNO}_{3}$. Leach residue with drop of 1:7 $\mathrm{HNO}_{3}$, transfer drop, and merge with it a drop of potassium mercuric thiocyanate solution. Greenish-yellow mosslike clumps and prisms prove copper. Test solution is tinged red, proving iron.

Domeykite $\left(\mathrm{Cu}_{3} \mathrm{As}\right)$.-Take mineral into solution with 1:1 $\mathrm{HNO}_{3}$. Add drop of ammonium molybdate solution to residue and slowly evaporate to dryness. cool and add drop of 1:7 $\mathrm{HNO}_{3}$ to residue to redissolve excess of ammonium molybdate. Tiny yellow isometric crystals prove arsenic. Transfer filtrate and merge with it a drop of potassium mercuric thiocyanate solution. Greenishyellow mosslike clumps and prisms prove copper.

Dufrenoysite $\left(2 \mathrm{PbS} . \mathrm{As}_{2} \mathrm{~S}_{3}\right)$. -Decompose mineral with $1: 1 \mathrm{HNO}_{3}$. Leach residue with drop of $1: 5 \mathrm{HCl}$, transfer drop, and evaporate almost to dryness. Leach residue from this evaporation with drop of ammonium molybdate soluHon. Evaporate slowly to dryness. Cool and add drop of 1:7 $\mathrm{HNO}_{3}$. Tiny yellow isometric crystals prove arsenic. Leach original residue with three successive drops of $\mathrm{H}_{2} \mathrm{O}$, transfer drops, and add to each a fragment of $\mathrm{KI}$. Yellow glistening hexagonal plates prove lead.

Dyscrasite $\left(\mathrm{Ag}_{3} \mathrm{Sb}\right)$.-Decompose mineral with 1:1 $\mathrm{HNO}_{3}$. Leach residue with drop of 1 per cent $\mathrm{HNO}_{3}$, transfer drop, and add fragment of ammonium bichromate. Red crystals prove silver. Leach original residue with 1:5 HCl, transfer drop, and add fragment of KI. Solution is colored yellow near fragment as it dissolves. Add fragment of $\mathrm{CsCl}$. Orange-colored hexagons and stars prove antimony.

Electrum (alloy of $\mathrm{Au}$ and $\mathrm{Ag}$ ). -Decompose mineral with 1:1 $\mathrm{HNO}_{8}$. Leach residue with drop of 1 per cent $\mathrm{HNO}_{3}$, transfer drop, and add fragment of ammonium bichromate. Red crystals prove silver. Exiamine original residue in obliquely reflected light. Usually the gold color of the particles of the residue is sufficient to identify the element. If not, dissolve residue in aqua regia and evaporate to dryness. Leach residue with drop of water, transfer drop, and merge with it a drop of pyridine-HBr solution. Strongly pleochroic red prisms prove gold.

Emplectite $\left(\mathrm{Cu}_{2} \mathrm{~S}_{\mathrm{S}} \mathrm{Bi}_{2} \mathrm{~S}_{3}\right)$.-Decompose mineral with 1:1 $\mathrm{HNO}_{3}$. Leach residue with drop of 1 per cent $\mathrm{HNO}_{3}$, transfer drop, and merge with it a drop of potassium mercuric thiocyanate solution. Greenish-yellow mosslike clumps and prisms prove copper. Leach original residue with two additional drops of 1 per cent $\mathrm{HNO}_{3}$ and one of water in order to remove as much of the remaining traces of copper as possible. Reject the three drops. Leach original residue 
with drop of 1:1 $\mathrm{HCh}_{\mathrm{h}}$ transfer drop, and add fragment of $\mathrm{KI}$. If any copper was carried over in the drop, it will precipitate as a light-yellow or gray amorphous powder. The solution turns yellow near the fragment as it dissolves. Then add fragment of $\mathrm{CsCl}$. Red hexagons prove bismuth.

Enargite $\left(\mathrm{Cu}_{2} \mathrm{~S} .4 \mathrm{CuS} . \mathrm{As}_{2} \mathrm{~S}_{3}\right)$.-Decompose mineral with $1: 1 \mathrm{HNO}_{3}$. Leach residue with 1:7 $\mathrm{HNO}_{3}$, transfer drop, and evaporate slowly over microflame. When nearly dry add drop of ammonium molybdate solution and again slowly evaporate. When dry, cool and add drop of 1:7 $\mathrm{HNO}_{3}$ to redissolve excess of ammonium molybdate. Tiny yellow isometric crystals prove arsenic. Transfer filtrate and merge with it a drop of potassium mercuric thiocyanate solution. Greenish-yellow mosslike clumps and prisms prove copper.

Eucairite $\left(\mathrm{Cu}_{2} \mathrm{Se} \cdot \mathrm{Ag}_{2} \mathrm{Se}\right)$.-Decompose mineral with 1:1 $\mathrm{HNO}_{3}$. Leach residue with drop of 1 per cent $\mathrm{HNO}_{3}$, transfer drop, and merge with it a drop of potassium mercuric thiocyanate solution. Greenish-yellow mosslike clumps and prisms prove copper, and an amorphous milky-white powder proves silver. Leach original residue with drop of 1:5 HCl, transfer drop, and merge with it a drop of stannous chloride solution. A brick-red amorphous precipitate proves selenium. Some elemental selenium will probably remain behind in the original residue as a red powder and can be best observed in oblique reflected light, using a 16-millimeter objective.

Famatinite $\left(\mathrm{Cu}_{2} \mathrm{~S} .4 \mathrm{CuS} . \mathrm{As}_{2} \mathrm{~S}_{3}\right)$.-Decompose mineral with $1: 1 \mathrm{HNO}_{3}$. Leach residue with drop of $1: 7 \mathrm{HNO}_{3}$, transfer drop, and evaporate slowly over microflame. When nearly dry add directly a drop of ammonium molybdate solution. Again evaporate over microflame. When dry, cool and add drup of 1: $7 \mathrm{HNO}_{3}$ to redissolve excess of ammonium molybdate. If arsenic is present in the mineral, tiny yellow isometric crystals will be seen near the periphery of the drop. Most specimens of famatinite contain more or less arsenic, and some idea of the proportion can be gained from the abundance of the ammonium arseni-molybdate precipitate. Transfer filtrate and test it for copper with potassium mercuric thiocyanate. Leach original residue with two drops of 1 per cent $\mathrm{HNO}_{3}$ and one of water to remove final traces of copper. Then leach original residue with drop of 1:5 HCl, transfer drop, and add fragment of KI. If any copper is present in the drop it will be precipitated as a light-yellow amorphous powder. The solution stains yellow near the KI fragment as it dissolves. Add fragment of $\mathrm{CsCl}$. The yellow color of the solution disappears, and orange-colored hexagons and stars form, proving antimony. Examine edge of drop in obliquely reflected light for amorphous orange-colored cesiumarsenic iodide precipitate.

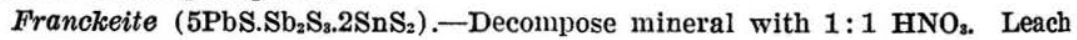
residue with 1:5 $\mathrm{HCl}$, transfer drop, and add fragment of $\mathrm{KI}$. Solution turns yellow near fragment as it dissolves. Add fragment of $\mathrm{CsCl}$ to solution. The yellow color of the solution disappears and orange-colored hexagons and colorless octahedra form, proving antimony and tin. Leach original residue with drop of water and evaporate over microflame in order to volatilize remainder of HCl. Cool, leach original residue with drop of water, transfer drop, and add fragment of KI. Yellow hexagons prove lead.

Franklinite $\left((\mathrm{Fe}, \mathrm{Zn}, \mathrm{Mn}) \mathrm{O} .(\mathrm{Fe}, \mathrm{Mn})_{2} \mathrm{O}_{3}\right)$.- Take mineral into solution with repeated applications of aqua regia. Leach residue with drop of 1:7 $\mathrm{HNO}_{3}$, transfer drop, and merge with it a drop of potassium mercuric thiocyanate solution. Solution turns red, and black prisms of indefinite form are precipitated. The composition of these is unknown, but both manganese and zinc may possibly be present. The test solution turns deep red, proving iron. Take additional fresh material into solution by means of aqua regia, leach residue 
with a drop of $1: 7 \mathrm{HNO}_{3}$, transfer drop, and test for manganese with sodium bismuthate fragments. A liberal amount of franklinite powder should be used in making the manganese test. Franklinite is extremely resistant to acid attack, but with persistence enough material can be taken into solution to give satisfactory tests.

Freibergite $\left(5(\mathrm{Cu}, \mathrm{Ag})_{2} \mathrm{~S} .2(\mathrm{Cu}, \mathrm{Fe}) \mathrm{S} .2 \mathrm{Sb}_{2} \mathrm{~S}_{3}\right)$.-Decompose mineral with $1: 1$ $\mathrm{HNO}_{3}$. Leach residue with 1 per cent $\mathrm{HNO}_{3}$, transfer drop, and merge with it a drop of potassium mercuric thiocyanate solution. A white curdy precipitate proves silver, and greenish-yellow mosslike clumps and prisms prove copper. Leach original residue with two successive drops of 1:7 $\mathrm{HNO}_{3}$ and one of water to remove final traces of copper. Then leach original residue with drop of 1:5 HCl, transfer drop, and add fragment of KI. Solution turns yellow Hear drop as it dissolves. If copper is present in drop it is precipitated here as an amorphous light-yellow or gray powder. Add fragment of $\mathrm{CsCl}$. Orangecolored hexagons and stars prove antimony.

Freieslebenite $\left(3 \mathrm{Ag}_{2} \mathrm{~S} .4 \mathrm{PbS} .3 \mathrm{Sb}_{2} \mathrm{~S}_{3}\right)$.-Decompose mineral with 1:1 $\mathrm{HNO}_{3}$. Leach residue with drop of 1 per cent $\mathrm{HNO}_{3}$. Transfer drop and add fragment of ammonium bichromate. Ied crystals, usually needle-shaped, prove silver, and an amorphous yellow precipitate proves lead. Leach original residue with a drop of 1:5 HCl. Transfer drop and add fragment of KI. Solution turns yellow near fragment. Then add fragment of $\mathrm{CsCl}$ to opposite side of drop. orange-colored hexagonal plates prove antimony. If necessary lead can be confirmed by adding a drop of $\mathrm{H}_{2} \mathrm{O}$ to the original residue, transferring drop, and adding a fragment of KI. Yellow glistening hexagonal plates prove lead.

Galena $(\mathrm{PbS})$.-Decompose mineral with 1:1 $\mathrm{HNO}_{3}$. Examine residue for lead nitrate lattice structure. Add drop of $1: 5 \mathrm{HCl}$ to residue. Remove $\mathrm{HCl}$ drop. Add drop of water to original residue and evaporate it to dryness in order to volatilize remaining trace of $\mathrm{HCl}$. Cool, add drop of water to original residue, transfer drop, and add to it a fragment of KI. Yellow hexagons prove lead.

Galenobismutite ( $\left.\mathrm{PbS}^{\mathrm{Bi}} \mathrm{B}_{2} \mathrm{~S}_{3}\right)$,-Decompose mineral with $1: 1 \mathrm{HNO}_{3}$. Examine residue for lead nitrate lattice. Leach reșidue with 1:5 HCl. Transfer drop and add fragment of KI. Solution turns yellow near fragment as it dissolves. Then add fragment of $\mathrm{CsCl}$. The yellow color disappears near fragment and red hexagons form, proving bismuth. Leach original residue with two successive drops of $1: 5 \mathrm{HCl}$, rejecting both. This will remove most of the remaining trace of bismuth. Then add three successive drops of water to original residue. Trausfer each drop to another place on slide and add fragment of KI. The first drop may not give a satisfactory test, owing to presence of remaining traces of bismuth. The second or third drop will give good yellow hexagons, which prove lead.

Geocronite $\left(5 \mathrm{PbS} \cdot \mathrm{Sb}_{2} \mathrm{~S}_{3}\right)$.-Decompose mineral with $1: 1 \mathrm{HNO}_{3}$. Examine residue for lattice structure, indicating lead. Leach residue with drop of 1:5 HCl. Transfer drop and add fragment of KI. Solution turns yellow near fragment as it dissolves. Then add fragment of cesium chloride. Orangecolored hexagons and stars prove antimony. Add drop of water to original residue and evaporate over flame to remove remaining traces of $\mathrm{HCl}$. Cool and again add drop of water to original residue. Transfer drop and add fragment of KI. Glistening yellow hexagons prove lead.

Germanite ( $\mathrm{Cu} \times \mathrm{GeSy}$ ).--Mineral very resistant to acid attack, but by repeated efforts, with aqua regia, enough can be broken down to give a copper test. Leach residue with 1: $7 \mathrm{HNO}_{3}$, transfer drop, and test for copper by merging with drop of potassium mercuric thiocyanate solution. There is no satisfactory microchemical test for germanium, an element allied chemically to silicon. 
Gersdorffite (NiAsS).-Decompose mineral with 1:1 $\mathrm{HNO}_{3}$. Leach residue with 1: $7 \mathrm{HNO}_{3}$, transfer drop, and slowly evaporate over microflame. When nearly dry, add a drop of ammonium molybdate and again slowly evaporate over microflame to dryness. Cool and add drop of 1: $7 \mathrm{HNO}_{3}$ to redissolve excess of ammonium molybdate. Tiny yellow isometric crystals prove arsenic. Transfer filtrate and merge with drop of potassium mercuric thiocyanate (method I). Small brown spherulites prove nickel. Cobalt and iron, if present, will be indicated at the same time. If desired, the dimethyl glyoxime test for nickel can be made by adding a drop of the reagent directly to the dried residue from the thiocyanate test.

Glaucodot ((Co,Fe)AsS).-Decompose mineral with $1: 1 \quad \mathrm{HNO}_{3}$. Leach residue with 1:7 $\mathrm{HNO}_{3}$, transfer drop, and evaporate slowly over microflame. When nearly dry, add drop of ammonium molybdate solution and again evaporate slowly to dryness over microflame. Cool and add drop of 1:7 $\mathrm{HNO}_{3}$ to redissolve excess of ammonium molybdate. Tiny yellow isometric crystals prove arsenic. Transfer filtrate and merge with drop of potassium mercuric thiocyanate solution. Test drop turns pink, proving iron, and dark indigo-colorel prisms are precipitated, oroving cobalt. Sometimes the prisms are so dark that the blue color is not apparent. It is best shown in strong oblique illumination.

Goethite $\left(\mathrm{Fe}_{2} \mathrm{O}_{3} \cdot \mathrm{H}_{2} \mathrm{O}\right)$. - Take mineral into solution in aqua regia. Repeated applications are necessary, as the mineral is resistant to chemical attack. Leach residue with a drop of $1: 5 \mathrm{HCl}$ and add $\mathrm{K}_{4} \mathrm{Fe}(\mathrm{CN})_{6}$ fragments. A blue amorphous powder proves iron.

Gold $(\mathrm{Au})$.- The color and sectility of gold are usually sufficient to identify it in polished section. If desired, however, a microchemical test can be made by dissolving mineral in aqua regia. The residue is dissolved in water, and the drop transferred and merged with a drop of pyridine-HBr solution. Strongly pleochroic (colorless to deep red) prisms prove gold.

Guanajuatite $\left(\mathrm{Bi}_{2}(\mathrm{Se}, \mathrm{S})_{3}\right)$.-Decompose mineral with $1: 1 \mathrm{HNO}_{3}$. Leach residue with drop of $1: 5 \mathrm{HCl}$, transfer drop, and add fragment of $\mathrm{CsCl}$. Thin iridescent colorless rhombs and knifeblade-shaped plates prove bismuth. Examine residue in obliquely reflected light. An agglutinated deep-red mass proves selenium. If necessary a microchemical test for selenium can be made by decomposing more mineral with $1: 1 \mathrm{HNO}_{3}$. The residue is leached with a drop of 1:5 HCl, and the drop is transferred and merged with a drop of stannous chloride solution (method I). A brick-red powder proves selenium.

Guitermanite (3PbS.As $\mathrm{S}_{3}$ ). -Decompose mineral with $1: 1 \mathrm{HNO}_{3}$. Examinè residue for lead nitrate lattice. Leach residue with $1: 5 \mathrm{HCl}$, transfer drop, and evaporate slowly over microflame. When nearly dry add drop of ammonium molybdate solution and again evaporate slowly over microflame. When dry, cool and add drop of $1: 7 \mathrm{HNO}_{3}$ to redissolve excess of ammonium molybdate. Tiny yellow isometric crystals prove arsenic. Add drop of water to original residue and evaporate to dryness over microflame to volatilize remaining trace of $\mathrm{HCl}$. Cool and add drop of water to original residue, transfer drop, and add fragment of KI. Yellow hexagons prove lead. The crystals may be somewhat distorted, owing to the presence of arsenic.

Hauerite $\left(\mathrm{MnS}_{2}\right)$.-Take mineral into solution in aqua regia. Take up residue in drop of 1:7 $\mathrm{HNO}_{3}$ and add fragments of sodium bismuthate. Solution turns pink or purple near fragments, proving manganese.

Hausmannite $\left(\mathrm{Mn}_{8} \mathrm{O}_{4}\right)$.-Take mineral into solution in aqua regia. Leach residue with a drop of 1:7 $\mathrm{HNO}_{3}$, transfer drop, and add fragments of sodium bismuthate. Solution turns pink or purple near fragments, proving manganese. 
Hematite $\left(\mathrm{Fe}_{2} \mathrm{O}_{3}\right)$.-This mineral is very resistant to chemical attack, but by repeated applications of aqua regia enough material can be taken into solution to give an iron test. Leach residue with a drop of 1:5 HCl, transfer drop, and add fragment of $\mathrm{K}_{4} \mathrm{Fe}(\mathrm{CN})_{\text {o. }}$ A blue amorphous precipitate proves iron.

Hessite $\left(\mathrm{Ag}_{2} \mathrm{Te}\right)$.-Decompose mineral with 1:1 $\mathrm{HNO}_{3}$. Leach residue with drop of 1 per cent $\mathrm{HNO}_{3}$, transfer drop, and add fragment of ammonium bichromate. Red crystals prove silver. Leach original residue with drop of 1:5 HCl. Any silver not already removed will be precipitated as a curdy white mass. Transfer filtrate and add fragment of $\mathrm{CsCl}$. Honey-yellow isometric crystals prove tellurium.

Jamesonite (4PbS.FeS.3 $\left.\mathrm{Sb}_{2} \mathrm{~S}_{3}\right)$. -Decompose mineral with $1: 1 \mathrm{HNO}_{3}$. Leach residue with drop of 1 per cent $\mathrm{HNO}_{3}$, transfer drop, and merge with drop of potassium mercuric thiocyanate solution. Test solution is tinged pink, proving presence of iron. Leach original residue with drop of 1:5 HCl. Transfer filtrate and add fragment of KI. Solution is colored light yellow near fragment as it dissolves. Add fragment of $\mathrm{CsCl}$. Orange-colored hexagons and stars prove antimony. Leach original residue with drop of water and evaporate to dryness to volatilize the remaining trace of $\mathrm{HCl}$. Cool again, add a drop of water to original residue, transfer drop, and add fragment of KI. Yellow hexagons prove lead.

Jordanite (4PbS.As $\mathrm{S}_{3}$ ).-Decompose mineral with $1: 1 \mathrm{HNO}_{3}$. Examine residue for lead nitrate lattice. Leach residue with 1:5 $\mathrm{HCl}$, transfer drop, and evaporate slowly over microflame. When nearly dry, add drop of ammonium molybdate solution and again evaporate slowly over microflame. When dry, cool and add drop of 1:7 $\mathrm{HNO}_{3}$ to redissolve excess of ammonium molybdate. Tiny yellow isometric crystals prove arsenic. Add drop of water to original residue and evaporate to dryness over microflame to volatilize remaining trace of $\mathrm{HCl}$. Cool and add drop of water to original residue, transfer drop, and add fragment of KI. Yellow hexagons prove lead. The crystals may be somewhat distorted, owing to the presence of arsenic.

Kallitite (Ni(Sb,Bi) S).-Decompose mineral with 1:1 $\mathrm{HNO}_{3}$. Leach residue with 1 per cent $\mathrm{HNO}_{3}$, transfer drop, evaporate to dryness, and add drop of dimethyl glyoxime solution. A pink precipitate proves nickel. Leach original residue with drop of 1:6 HCl, transfer drop, and add fragment of KI. Solution turns yellow. Add fragment of $\mathrm{CsCl}$. Red hexagons prove bismuth. Leach original residue with another drop of $1: 5 \mathrm{HCl}$ and again repeat cesium double iodide test. Both red and orange-colored hexagons will form, proving that antimony is also present.

Keeleyite $\left(\mathrm{PbS} \cdot \mathrm{Sb}_{2} \mathrm{~S}_{3}\right)$.-Decompose mineral with 1:1 $\mathrm{HNO}_{3}$. Examine residue for lattice structure, indicating lead. Leach residue with drop of 1:5 HCl. Transfer drop and add fragment of KI. Solution turns yellow near fragment as it dissolves. Then add fragment of cesium chloride. Orangecolored hexagons and stars prove antimony. Add drop of water to original residue and evaporate over flame to remove remaining traces of $\mathrm{HCl}$. Cool and again add drop of water to original residue. Transfer drop and add fragment of KI. Glistening yellow hexagons prove lead.

Kermesite $\left(\mathrm{Sb}_{2} \mathrm{~S}_{2} \mathrm{O}\right)$. - The $\mathrm{KOH}$ etch test is very characteristic. The presence of antimony can be confirmed microchemically as follows: Decompose mineral with aqua regia. Leach residue with drop of $1: 5 \mathrm{HCl}$, transfer drop, and add fragment of KI. Solution turns yellow near fragment as it dissolves. Add fragment of $\mathrm{CsCl}$ to solution. Orange-colored stars and hexagons, the stars predominating, prove antimony. 
Klaprothite $\left(3 \mathrm{Cu}_{2} \mathrm{~S} .2 \mathrm{Bi}_{2} \mathrm{~S}_{3}\right)$.-Decompose mineral with $1: 1 \mathrm{HNO}_{3}$. Leach residue with drop of 1 per cent $\mathrm{HNO}_{3}$, transfer drop, and merge with it a drop of potassium mercuric thiccyanate solution. Greenish-yellow mosslike clumps and prisms prove copper. Leach original residue with two additional drops of 1 per cent $\mathrm{HNO}_{3}$ and one of water in order to remove as much of the remaining traces of copper as pos ible. Reject the three drops. Leach original residue with drop of $1: 5 \mathrm{HCl}$, transfer drop, and add fragment of $\mathrm{KI}$. If any copper was carried over in the drop, it will be precipitated as a lightyellow or gray amorphous powder. The solution turns yellow near the frag. ment as it dissolves. Then add fragment of $\mathrm{CsCl}$. Red hexagons prove bismuth.

Klockmannite (CuSe).-Take mineral into solution in $1: 1 \mathrm{HNO}_{3}$. Leach residue with a drop of 1 per cent $\mathrm{HNO}_{3}$, transfer drop, and test for copper by merging with a drop of potassium mercuric thiocyanate reagent. Greenishyellow mosslike clumps and prisms prove copper. Leach original residue with drop of 1:5 HCl, transfer drop, and merge with drop of stannous chloride reagent. An amorphous brick-red precipitate proves selenium.

Kremnerite ( $\left.(\mathrm{Au}, \mathrm{Ag}) \mathrm{Te}_{2}\right)$.-Decompose mineral with 1:1 $\mathrm{HNO}_{3}$. One or two applications of acid suffice. The residue is a white curdy powder. Leach residue with 1:5 HCl. The white curdy residue goes into solution at once. Transfer drop and add a fragment of CsCl. Honey-yellow isometric crystals prove tellurium. Gold remains in the original residue in small metallic grains and lumps. Usually examination in oblique reflected light with a 16-millimeter objective suffices to identify the gold. If the appearance of the residue is not sufficiently characteristic, add drop of aqua regia to residue and evaporate to dryness. An orange-colored residue of gold chloride remains. Dissolve this in water, transfer drop, and merge with it a drop of pyridine- $\mathrm{HBr}$ reagent. Strongly pleochroic (colorless to deep red) prisms prove gold. Silver remains in the original residue as a curdy white chloride. Leach it with two or three successive drops of 1:5 HCl, to remove remaining traces of gold and tellurium, then one of water to remove $\mathrm{HCl}$. Then add to original residue a drop of concentrated $\mathrm{NH}_{4} \mathrm{OH}$. Observe with an 8-millimeter objective. In a few seconds tiny colorless isometric crystals of $\mathrm{AgCl}$ will be seen floating in the ammonia.

Lengenbachite (6PbS. $\left.(\mathrm{Ag}, \mathrm{Cu})_{2} \mathrm{~S} .2 \mathrm{AsS}_{3}\right)$. -Decompose mineral with 1:1 $\mathrm{HNO}_{3}$. Leach residue with drop of 1 per cent $\mathrm{HNO}_{3}$, transfer drop, and merge with drop of potassium mercuric thiocyanate reagent. An amorphous finely divided milk-white precipitate proves silver, and greenish-yellow mosslike clumps and prisms prove copper. Leach original residue with drop of 1:5 $\mathrm{HCl}$, take up drop, and reject. Leach original residue with three successive drops of $\mathrm{H}_{2} \mathrm{O}$ and add to each a tiny fragment of KI. Yellow glistening hexagonal plates prove lead. Some arsenic and copper may get into the first drop and hinder the formation of characteristic $\mathrm{PbI}_{2}$ plates, but the second or third. drop should give a good test for lead. To test for arsenic take additional fresh mineral into solution in 1:1 $\mathrm{HNO}_{3}$. Leach residue with drop of 1:7 $\mathrm{HNO}_{8}$. transfer drop, and slowly evaporate. When nearly dry, add drop of ammonium molybdate reagent and slowly evaporate to dryness. Cool and add a drop of 1: $7 \mathrm{HNO}_{3}$ to residue. Tiny yellow isometric crystals prove arsenic.

Lencopyrite $\left(\mathrm{Fe}_{3} \mathrm{As}_{4}\right)$. -Decompose mineral with 1:1 $\mathrm{HNO}_{3}$. The residue usually has a tinge of yellow or orange. Leach residue with drop of 1:7 $\mathrm{HNO}_{3}$, transfer drop, and evaporate slowly by passing in and out of microflame. When nearly dry add directly a drop of ammonium molybdate solution and again heat slowly over microflame. When dry, cool and add drop of $1: 7 \mathrm{HNO}_{3}$ to redissolve excess ammonium molybdate. Examine under microscope. Tiny yel- 
low isometric crystals prove arsenic. Take up filtrate and transfer to another place on slide. Merge with it a drop of potassium mercuric thiocyanate solution. A red coloration of test solution proves iron.

Limonite $\left(\mathrm{Fe}_{2} \mathrm{O}_{3} \cdot \mathrm{nH}_{2} \mathrm{O}\right)$. -Take mineral into solution in aqua regia. Repeated applications of acid are necessary, as limonite is resistant to chemical attack. Add drop of 1:5 $\mathrm{HCl}$ to residue and add fragment of $\mathrm{K}_{4} \mathrm{Fe}(\mathrm{CN})_{8}$ to drop. An amorphous blue precipitate proves iron.

Linnaeite $\left(\mathrm{Co}_{3} \mathrm{~S}_{4}\right)$.-Decompose mineral with 1:1 $\mathrm{HNO}_{3}$. Leach residue with 1:7 $\mathrm{HNO}_{3}$, transfer dro thiocyanate solution. Indigo-blue prisms prove cobalt. Brownish nickel spherulites will usually come down in addition to the cobalt precipitate.

Livingstonite (HgS.2Sb ${ }_{2} \mathrm{~S}_{3}$ ). -Decompose mineral with aqua regia. Examine residue for mercuric chloride lattice structure. Leach residue with drop of 1 per cent $\mathrm{HNO}_{3}$, transfer drop, and add fragment of cobalt nitrate. When dissolved add fragment of KCNS. Indigo-blue arborescent forms and prisms prove mercury. Leach original residue with drop of 1:5 HCl. Transfer drop and add fragment of KI. Solution turns yellow near fragment as it dissolves. Add fragment of $\mathrm{CsCl}$. Orange-colored hexagons and stars prove antimony.

Löllingite $\left(\mathrm{FeAs}_{2}\right)$.-Dissolve mineral in 1:1 $\mathrm{HNO}_{6}$. The residue usually has a tinge of yellow or orange. Leach residue with drop of 1:7 $\mathrm{HNO}_{3}$, transfer drop, and evaporate slowly by passing in and out of microflame. When nearly dry add directly a drop of ammonium molybdate solution and again heat slowly over microflame. When dry, cool and add drop of 1:7 $\mathrm{HNO}_{3}$ to redissolve excess ammoniumm molybdate. Examine under microscope. Tiny yellow isometric (rystals prove arsenic. Take up filtrate and transfer to another place on slide. Merge with it a drop of potassium mercuric thiocyanate solution. A red coloration of test solution proves iron.

Lorandite $\left(\mathrm{Tl}_{2} \mathrm{~S} . \mathrm{As}_{2} \mathrm{~S}_{3}\right)$. - A fragment of mineral tested in a Bunsen flame gave a vivid green (thallium) coloration to the flame. Fragment was held in a very small loop on a platinum wire that also held a small drop of $\mathrm{HCl}$. Under the same conditions copper gives a blue flame. Arsenic is tested for as follows: Decompose mineral with aqua regia. Leach residue with drop of 1: $7 \mathrm{HNO}_{3}$, transfer drop, and slowly evaporate over microflame. When nearly dry add drop of ammonium molybdate solution and again slowly evaporate, this time to dryness. Cool and add drop of 1:7 $\mathrm{HNO}_{s}$ to redissolve excess of ammonium molybdate. Tiny yellow isometric crystals prove arsenic.

Magnetite $\left(\mathrm{Fe}_{3} \mathrm{O}_{4}\right)$.- This mineral is very resistant to chemical attack, but by repeated applications of aqua regia enough material can be taken into solution to give an iron test. Leach residue with a drop of $1: 5 \mathrm{HCl}$, transfer drop, and add fragment of $\mathrm{K}_{4} \mathrm{Fe}(\mathrm{CN})_{8}$. A blue amorphous precipitate proves iron.

Manganite $\left(\mathrm{Mn}_{2} \mathrm{O}_{3}\right)$.-Take mineral into solution in aqua regia. Take up residue in drop of 1:7 $\mathrm{HNO}_{3}$ and add fragments of sodium bismuthate. Solution turns pink or purple near fragments, proving manganese.

Marcasite $\left(\mathrm{FeS}_{2}\right)$.-Decompose mineral with 1:1 $\mathrm{HNO}_{3}$. Residue is colored yellow or orange. Leach residue with drop of 1:5 HCl, transfer drop, and add fragment of $\mathrm{K} F e(C N)_{\text {. }}$. A blue amorphous precipitate proves iron.

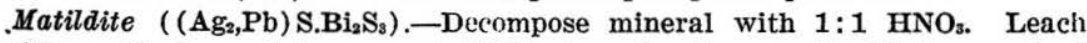
residue with drop of 1 per cent $\mathrm{HNO}_{3}$, transfer drop, and add fragment of ammonium bichromate. A yellow amorphous precipitate proves lead, and red crystals prove silver. Leach original residue with drop of 1:5 riCl. Transfer drop and add fragment of KI. Solution stains yellow near ragment as it dissolves. Then add fragment of $\mathrm{CsCl}$. Red hexagons prove bismuth. Leach original residue with two successive drops of $1: 5 \mathrm{HCl}$ and reject both. This 
will remove nearly all the remainder of the bismuth. Then leach original residue with three successive drops of water. Transfer each drop to another place on slide and test with fragment of $\mathrm{KI}$. The first drop may not give a satisfactory test for lead, owing to the presence of remaining traces of lead and bismuth, but the second or third drop will yield hexagonal plates which prove lead.

Maucherite $\left(\mathrm{Ni}_{3} \mathrm{As}_{2}\right)$.-Take mineral into solution with 1:1 $\mathrm{HNO}_{3}$. Leach residue with drop of 1:7 $\mathrm{HNO}_{3}$. Transfer drop and evaporate slowly over microflame. When nearly dry, add drop of ammonium molybdate and again evaporate slowly over microflame, this time to dryness. Cool and add drop of $1: 7 \mathrm{HNO}_{3}$ to redissolve excess of ammonium molybdate. Tiny yellow isometric crystals prove arsenic. Transfer filtrate and evaporate to dryness. Cool and add a drop of dimethyl glyoxime solution to residue. A pink precipitate proves nickel.

Melaconite $(\mathrm{CuO})$.-Dissolve mineral in 1:1 $\mathrm{HNO}_{3}$. Take up residue with drop of 1:7 $\mathrm{HNO}_{3}$, transfer drop, and merge with drop of potassium mercuric thiocyanate reagent. Greenish-yellow mosslike clumps and prisms prove copper.

Melonite $\left(\mathrm{Ni}_{2} \mathrm{Te}_{3}\right)$.-Dissolve mineral in 1:1 $\mathrm{HNO}_{3}$. Take up residue in drop of 1:5 HCl. By means of capillary tube divide this drop into two equal parts. Test one part by adding fragment of CsCl. Honey-yellow isometric crystals prove tellurium. Evaporate other part to dryness and add drop of dimethyl glyoxime reagent. A pink precipitate proves nickel.

Meneghinite (4PbS.Sb $\left.\mathrm{S}_{3}\right)$.-Decompose mineral with 1:1 $\mathrm{HNO}_{3}$. Examine residue for lattice structure, indicating lead. Leach residue with drop of 1:5 HCl. Transfer drop and add fragment of KI. Solution turns yellow near fragment as it dissolves. Then add fragment of cesium chloride. Orange-colored hexagons and stars prove antimony. Add drop of water to original residue and evaporate over flame to remove remaining traces of $\mathrm{HCl}$. Cool and again add drop of water to original residue. Transfer drop and add fragment of KI. Glistening yellow hexagons prove lead.

Metacinnabarite (HgS).-Decompose mineral by means of aqua regia. Examine residue for mercuric chloride lattice structure, Leach residue with drop of 1 per cent $\mathrm{HNO}_{3}$ and transfer drop. Add fragment of $\mathrm{Co}\left(\mathrm{NO}_{3}\right)_{2}$. When dissolved add fragment of potassium thiocyanate. Indigo-blue prisms and branching forms of cobalt mercuric thiocyanate prove mercury.

Miargyrite $\left(\mathrm{Ag}_{2} \mathrm{~S}_{2} \mathrm{Sb}_{2} \mathrm{~S}_{3}\right)$.-Decompose mineral with 1:1 $\mathrm{HNO}_{3}$. Leach residue with 1 per cent $\mathrm{HNO}_{3}$, transfer drop, and add fragment of ammonium bichromate. Red crystals prove silver. Leach original residue with a drop of 1: $5 \mathrm{HCl}$, transfer drop, and add fragment of KI. Solution turns yellow near the fragment as it dissolves. Then add a fragment of $\mathrm{CsCl}$. Orange-colored hexagons and stars prove antimony.

Millerite (NiS).-Decompose mineral with $1: 1 \mathrm{HNO}_{3}$. Leach residue with drop of 1:7 $\mathrm{HNO}_{3}$, transfer drop, and evaporate to dryness. To the residue from this evaporation add a drop of dimethyl glyoxime solution. A pink precipitate proves nickel.

Nagyagite $(\mathrm{Pb}, \mathrm{Au}, \mathrm{Te}, \mathrm{S})$ ?.-Decompose mineral with $1: 1 \mathrm{HNO}_{3}$. Leach residue with drop of 1:5 HCl. Transfer drop and add fragment of $\mathrm{CsCl}$. Honeyyellow isometric crystals prove tellurium. The crystals are smaller with nagyagite than with other tellurides, for some unknown reason. Leach original residue with a drop of water and evaporate to dryness over microflame in order to volatilize remaining trace of $\mathrm{HCl}$. Cool and add another drop of water to original residue. Transfer drop and add fragment of KI. Yellow hexagons prove lead. The writer was unable to get a test for gold by the pyridine-HBr method. 
Naumanite $\left(\mathrm{Ag}_{2}, \mathrm{~Pb}\right) \mathrm{Se}$-Decompose mineral with 1:1 $\mathrm{HNO}_{3}$. Leach residue with 1 per cent $\mathrm{HNO}_{3}$, transfer drop, and add a fragment of ammonium bichromate. A yellow amorphous precipitate proves lead, and red crystals prove silver. Add drop of 1:5 $\mathrm{HCl}$ to original residue. Any silver remaining is converted into the white flocculent chloride. Transfer filtrate and merge it with a drop of stannous chloride solution. A brick-red amorphous precipitate proves selenium. Leach originai residue with a drop of water and evaporate over a microflame in order to volatilize the remaining $\mathrm{HCl}$. Cool, again leach original residue with a drop of water, transfer drop, and add a fragment of KI. Yellow hexagons prove lead. Naumanite and aguilarite can be distinguished only by a microchemical test for lead.

Nicoolite (NiAs).-Take mineral into solution with 1:1 $\mathrm{HNO}_{3}$. Leach residue with drop of 1:7 $\mathrm{HNO}_{3}$. Transfer drop and evaporate slowly over microflame. When nearly dry, add drop of ammonium molybdate and again evaporate slowly over microflame, this time to dryness. Cool and add drop of 1:7 $\mathrm{HNO}_{3}$ to redissolve excess of ammonium molybdate. Tiny yellow isometric crystals prove arsenic. Transfer filtrate and evaporate to dryness. Cool and add a drop of dimethyl glyoxime solution to residue. A pink precipitate proves nickel.

Orpiment $\left(\mathrm{As}_{2} \mathrm{~S}_{3}\right)$. -Decompose mineral with 1:1 $\mathrm{HNO}_{3}$. Leach residue with 1:7 $\mathrm{HNO}_{3}$, transfer drop, and evaporate slowly over microflame. When nearly dry, add drop of ammonium molybdate solution and again evaporate slowly over microflame, this time to dryness. Cool and add drop of 1:7 $\mathrm{HNO}_{3}$ to dissolve excess of ammonium molybdate. Tiny yellow isometric crystals prove arsenic.

Owyheeite ( $\left.8 \mathrm{PbS} .2 \mathrm{Ag}_{2} \mathrm{~S}_{2} \mathrm{SSb}_{2} \mathrm{~S}_{3}\right)$. -Decompose mineral with 1:1 $\mathrm{HNO}_{3}$. Leach residue with drop of 1 per cent $\mathrm{HNO}_{3}$, transfer drop, and add tiny fragment of ammonium bichromate. An amorphous yellow precipitate proves lead, and red needlelike crystals prove silver. Leach original residue with drop of 1: $5 \mathrm{HCl}$. Transfer drop and add tiny fragment of $\mathrm{KI}$ to one side of drop. Solution turns yellow near fragment. Then add fragment of $\mathrm{CsCl}$ to other side of drop. Orange-colored hexagons and stars prove antimony. If necessary the presence of lead can be confirmed by leaching original residue with a drop of water, transferring drop, and testing for lead with fragment of $\mathrm{KI}$.

Pearcite $\left(8 \mathrm{Ag}_{2} \mathrm{~S} . \mathrm{As}_{2} \mathrm{~S}_{3}\right)$.-Decompose mineral with 1:1 $\mathrm{HNO}_{3}$. Leach residue with 1 per cent $\mathrm{HNO}_{3}$, transfer drop, and merge with a drop of potassium mercuric thiocyanate solution (method I). An amorphous white precipitate proves silver. Usually there is also a significant proportion of copper in pearcite, which is the reason for choosing the thiocyanate test in preference to the bichromate test. Much of the arsenic acid was also carried from the original residue by the 1 per cent $\mathrm{HNO}_{3}$ drop. Usually there is enough arsenic remaining to give a satisfactory test. Leach original residue with 1:7 $\mathrm{HNO}_{3}$, transfer drop, and evaporate slowly over microflame. When nearly dry, add drop of ammonium molybdate solution and again evaporate slowly over microflame, this time to dryness. Cool and add drop of $1: 7 \mathrm{HNO}_{3}$ to redissolve excess of ammonium molybdate. Tiny yellow isometric crystals prove arsenic.

Pentlanaite $((\mathrm{Ni}, \mathrm{Fe}) \mathrm{S})$.-Decompose mineral with 1:1 $\mathrm{HNO}_{3}$. Leach residue with 1: $7 \mathrm{HNO}_{3}$, transfer drop, and evaporate to dryness. Add drop of 1 per cent $\mathrm{NH}_{4} \mathrm{OH}$ to residue. A yellow gelatinous precipitate of $\mathrm{Fe}(\mathrm{OH})_{3}$ proves iron. Allow the $\mathrm{NH}_{4} \mathrm{OH}$ to evaporate in air. When nearly dry add directly a drop of dimethyl glyoxime solution. A pink (nickel) precipitate will form on top of the gelatinous ferric hydroxide coating.

Petzite ( $\left.(\mathrm{Ag}, \mathrm{Au})_{2} \mathrm{Te}\right)$.-Decompose mineral with 1:1 $\mathrm{HNO}_{3}$. Leach residue with 1 per cent $\mathrm{HNO}_{3}$, transfer drop, and add a fragment of ammonium bi15122-31-14 
chromate. Red crystals prove silver. Leach original residue with a drop of 1:5 $\mathrm{HCl}$, transfer drop, and add a fragment of $\mathrm{CsCl}$. Honey-yellow isometric crystals prove tellurium. Examine original residue in oblique illumination with a 16-millimeter objective. Usually metallic gold particles in the residue are sufficiently distinctive in appearance to make a confirmatory test unnecessary. If necessary it can be made as follows: The original residue is dissolved in aqua regia and the solution evaporated to dryness. The residue, which is orange-colored to light brown, is dissolved in water, the drop transferred and merged with a drop of pyridine-HBr solution. Strongly pleochroic (colorless to deep red) prisms prove gold.

Plagionite (5PbS.4 $\left.\mathrm{Sb}_{2} \mathrm{~S}_{3}\right)$.-Decompose mineral with $1: 1 \mathrm{HNO}_{3}$. Examine residue for lattice structure, indicating lead. Leach residue with drop of $1: 5$ HCI. Transfer drop and add fragment of KI. Solution turns yellow near fragment as it dissolves. Then add fragment of cesium chloride. Orangecolored hexagons and stars prove antimony. Add drop of water to original residue and evaporate over flame to remove remaining traces of $\mathrm{HCl}$. Cool and again add drop of water to original residue. Transfer drop and add fragment of KI. Glistening yellow hexagons prove lead.

Plessite ( (Ni,Fe) AsS ).-Decompose mineral with 1:1 $\mathrm{HNO}_{3}$. Leach residue with a drop of $1: 7 \mathrm{HNO}_{3}$, transfer drop, and evaporate it slowly over microflame. When nearly dry add drop of ammonium molybdate solution and again evaporate slowly, this time to dryness. Cool and add drop of 1:7 $\mathrm{HNO}_{3}$. Tiny yellow isometric crystals prove arsenic. Transfer filtrate and merge with drop of potassium mercuric thiocyanate. Test solution turns red, proving iron, and small brown spherulites are precipitated, proving nickel. If desired confirm nickel test by allowing the drop containing the nickel spherulites to evaporate to dryness and then adding a drop of dimethyl glyoxime solution. A pink precipitate confirms the presence of nickel.

Polybasite $\left(8 \mathrm{Ag}_{2} \mathrm{~S}_{2} \mathrm{Sb}_{2} \mathrm{~S}_{3}\right)$.-Decompose mineral with $1: 1 \mathrm{HNO}_{3}$. Leach residue with drop of 1:7 $\mathrm{HNO}_{3}$, transfer drop, and merge with drop of potassium mercuric thiocyanate. A white curdy amorphous precipitate proves silver, and just behind it a copper precipitate usually forms when testing polybasite. Leach original residue with drop of $1: 5 \mathrm{HCl}$, transfer drop, and add fragment of $\mathrm{KI}$. The solution turns yellow near the fragment as it dissolves. Then add fragment of CsCl. Orange-colored hexagons and stars prove antimony.

Polydymite $\left(\mathrm{Ni}_{3} \mathrm{~S}_{4}\right)$.-Decompose mineral with $1: 1 \mathrm{HNO}_{3}$. Leach residue with drop of 1:7 $\mathrm{HNO}_{3}$, transfer drop, and evaporate to dryness. To the residue from this evaporation add a drop of dimethyl glyoxime solution. A pink precipitate proves nickel.

Proustite $\left(3 \mathrm{Ag}_{2} \mathrm{~S} \mathrm{As}_{2} \mathrm{~S}_{3}\right)$. -Decompose mineral with $1: 1 \mathrm{HNO}_{3}$. Leach residue with drop of 1 per cent $\mathrm{HNO}_{3}$, transfer drop, and add fragment of ammonium bichromate. Red crystals prove silver. Some of the arsenic was removed from the original residue in the drop of 1 per cent $\mathrm{HNO}_{3}$. Enough is usually left in the original residue to give a satisfactory arsenic test. Leach original residue with drop of 1:7 $\mathrm{HNO}_{3}$, transfer drop, and evaporate slowly over microflame. When nearly dry, add drop of ammonium molybdate solution and again evaporate slowly over microflame, this time to dryness. Gool and add drop of 1:7 $\mathrm{HNO}_{3}$ to dissolve excess ammonium molybdate. Tiny yellow isometric crystals prove arsenic.

Psilomelane $\left(\mathrm{Mn}_{2} \mathrm{MnO}_{5}\right)$.-Take mineral into solution in aqua regia. Take up residue in drop of 1: $7 \mathrm{HNO}_{3}$ and add fragments of sodium bismuthate. Solution turns pink or purple near fragments, proving manganese.

Pyrargyrite $\left(3 \mathrm{Ag}_{2} \mathrm{~S}_{2} \mathrm{Sb}_{2} \mathrm{~S}_{3}\right)$.-Decompose mineral with $1: 1 \mathrm{HNO}_{3}$. Leach residue with 1 per cent $\mathrm{HNO}_{3}$, transfer drop, and add fragment of ammonium 
bichromate. Red crystals prove silver. Leach original residue with a drop of 1:5 HCl, transfer drop, and add fragment of KI. Solution turns yellow near the fragment as it dissolves. Then add a fragment of $\mathrm{CsCl}$. Orangecolored hexagons and stars prove antimony.

Pyrolusite $\left(\mathrm{MnO}_{2}\right)$.-Take mineral into solution in aqua regia. Take up residue in drop of $1: 7 \mathrm{HNO}_{3}$ and add fragments of sodium bismuthate. Solution turns pink or purple near fragments, proving manganese.

Pyrrhotite $\left(\mathrm{FeS}_{1^{+}}\right)$.-Decompose mineral with 1:1 $\mathrm{HNO}_{3}$. Residue is colored yellow or orange. Leach residue with drop of 1:5 HCl, transfer drop, and add fragment of $\mathrm{K}_{4} \mathrm{Fe}(\mathrm{CN})_{0}$. A blue amorphous precipitate proves iron.

Pyrite $\left(\mathrm{FeS}_{2}\right)$.-Decompose mineral with $1: 1 \mathrm{HNO}_{3}$. Residue is colored yellow or orange. Leach residue with drop of $1: 5 \mathrm{HCl}$, transfer drop, and add fragment of $\mathrm{K}_{4} \mathrm{Fe}(\mathrm{CN})_{6}$. A blue amorphous precipitate proves iron.

Rammelsbergite $\left(\mathrm{NiAs}_{2}\right)$. - Take mineral into solution with $1: 1 \mathrm{HNO}_{3}$. Leach residue with drop of $1: 7 \mathrm{HNO}_{3}$. Transfer drop and evaporate slowly over microflame. When nearly dry, add drop of ammonium molybdate and again evaporate slowly over microflame, this time to dryness. Cool and add drop of $1: 7 \mathrm{HNO}_{3}$ to redissolve excess of ammonium molybdate. Tiny yellow isometric crystals prove arsenic. Transfer filtrate and evaporate to dryness. Cool and add a drop of dimethyl glyoxime solution to residue. A pink precipitate proves nickel.

Rathite (3PbS.2As $\left.\mathrm{S}_{3}\right)$.-Decompose mineral with $1: 1 \mathrm{HNO}_{3}$. Examine residue for lead nitrate lattice. Leach residue with 1:5 $\mathrm{HCl}$, transfer drop, and evaporate slowly over microflame. When nearly dry, add drop of ammonium molybdate solution and again evaporate slowly over microflame. When dry, cool and add drop of 1:7 $\mathrm{HNO}_{3}$ to redissolve excess of ammonium molybdate. Tiny yellow isometric crystals prove arsenic. Add three successive drops of water to original residue. Transfer each drop to another place on slide and add fragment of $\mathrm{KI}$. The first drop may not give a satisfactory test for lead, owing to the presence of remaining traces of As, but the second or third drop should give yellow hexagons, possibly somewhat distorted, proving lead.

Realgar (AsS).-Decompose mineral with 1:1 $\mathrm{HNO}_{3}$. Leach residue with 1: $7 \mathrm{HNO}_{3}$, transfer drop, and evaporate slowly over microflame. When nearly dry, add drop of ammonium molybdate solution and again evaporate slowly over microflame, this time to dryness. Cool and add drop of 1:7 $\mathrm{HNO}_{3}$ to dissolve excess of ammonium molybdate. Tiny yellow isometric crystals prove arsenic.

Rezbanyite (2PbS.3Bi $\left.\mathrm{B}_{2}\right)$.-Decompose mineral with $1: 1 \mathrm{HNO}_{3}$. Examine residue for lead nitrate lattice. Leach residue with 1:5 HCl. Transfer drop and add fragment of KI. Solution turns yellow near fragment as it dissolves. Then add fragment of $\mathrm{CsCl}$. The yellow color disappears near fragment, and red hexagons form, proving bismuth. Leach original residue with two successive drops of $1: 5 \mathrm{HCl}$, rejecting both. This will remove most of the remaining trace of bismuth. Then add three successive drops of water to original residue, transfer each drop to another place on slide, and add fragment of KI. The first drop may not give a satisfactory test for lead, owing to the presence of remaining traces of $\mathrm{Bi}$ and $\mathrm{HCl}$. The second or third drop should yield yellow hexagons, proving lead.

Rickardite ( $\left.\mathrm{Cu}_{x} \mathrm{Te}\right)$.-Take mineral into solution with 1:1 $\mathrm{HNO}_{3}$. Leach residue with 1 per cent $\mathrm{HNO}_{3}$, transfer drop, and merge with drop of potassium mercuric thiocyanate solution. Greenish-yellow mosslike aggregates and prisms prove copper. Leach original residue with 1:5 HCl, transfer drop, and add fragment of $\mathrm{CsCl}$. Honey-yellow isometric crystals prove tellurium. 
Schapbachite $\left(\mathrm{Ag}_{2} \mathrm{~S} . \mathrm{PbS}_{\mathrm{B}} \mathrm{Bi}_{2} \mathrm{~S}_{3}\right)$. -Decompose mineral with 1:1 $\mathrm{HNO}_{3}$. Leach residue with drop of 1 per cent $\mathrm{HNO}_{3}$, transfer drop, and add fragment of ammonium bichromate. A yellow amorphous precipitate proves lead, and red crystals prove silver. Leach original residue with drop of 1:5 HCl, transfer drop, and add fragment of KI to one side of drop. Solution turns yellow near drop. Then add fragment of $\mathrm{CsCl}$ to opposite side of drop. Red hexagons prove $\mathrm{Bi}$. If necessary the presence of lead can be confirmed as follows: Leach original residue with three successive drops of 1:5 HCl, rejecting each. This removes most of remaining traces of bismuth. Then leach original residue with successive drops of $\mathrm{H}_{2} \mathrm{O}$, testing each with a fragment of $\mathrm{KI}$. The first drop may give an indefinite result, owing to a trace of bismuth, but this tends to hydrolyze in the water, and the second or third drop will give good $\mathrm{PbI}_{2}$ hexagonal plates.

Semseyite (9PbS.4 $\left.\mathrm{Sb}_{2} \mathrm{~S}_{3}\right)$. -Decompose mineral with $1: 1 \mathrm{HNO}_{3}$. Examine residue for lattice structure, indicating lead. Leach residue with drop of $1: 5$ HCl. Transfer drop and add fragment of KI. Solution turns yellow near fragment as it dissolves. Then add fragment of cesium chloride. Orangecolored hexagons and stars prove antimony. Add drop of water to original residue and evaporate over flame to remove remaining traces of $\mathrm{HCl}$. Cool and again add drop of water to original residue. Transfer drop and add fragment of KI. Glistening yellow hexagons prove lead.

Siegenite $\left((\mathrm{Ni}, \mathrm{Co})_{3} \mathrm{~S}_{4}\right)$.-Decompose mineral with 1:1 $\mathrm{HNO}_{3}$. Leach residue in drop of 1: $7 \mathrm{HNO}_{3}$, transfer drop, and merge with drop of potassium mercuric thiocyanate reagent. If solution turns red, iron is present. Brown spherulites prove nickel, and blue spherulites prove cobalt.

Silver (Ag).-Take into solution with 1:1 $\mathrm{HNO}_{3}$. Add drop of 1 per cent $\mathrm{HNO}_{3}$ to residue. Transfer drop and add fragment of ammonium bichromate. Red crystals prove silver.

Skutterudite ( $\left.(\mathrm{Co}, \mathrm{Ni}) \mathrm{As}_{3}\right)$. -Take mineral into solution with 1:1 $\mathrm{HNO}_{3}$. Leach residue with drop of 1:7 $\mathrm{HNO}_{3}$, transfer drop, and evaporate slowly over microflame. When nearly dry add drop of ammonium molybdate solution and again slowly evaporate. When dry, cool and add drop of 1:7 $\mathrm{HNO}_{3}$ solution to redissolve excess of ammonium molybdate. Tiny yellow isometrie crystals prove arsenic. Transfer filtrate and merge with drop of potassium mercuric thiocyanate solution. Brown spherulites prove nickel and blue prisms cobalt.

Smaltite-chloanthite $\left((\mathrm{Co}, \mathrm{Ni}) \mathrm{As}_{2}\right)$. - Take mineral into solution in aqua regia. Take up residue in a drop of 1:7 $\mathrm{HNO}_{3}$ and slowly evaporate drop. When nearly dry, add drop of ammonium molybdate reagent and slowly evaporate to dryness, cool, add drop of 1:7 $\mathrm{HNO}_{3}$ to residue, and examine with an 8-millimeter objective. Tiny yellow isometric crystals prove arsenic. Transfer flltrate and merge with drop of potassium mercuric thiocyanate reagent. If solution turns red, iron is present. Brown spherulites prove nickel; blue spherulites, cobalt.

Sphalerite $(\mathrm{ZnS})$.-Decompose mineral with aqua regia. Leach residue with drop of 1:7 $\mathrm{HNO}_{3}$, transfer drop, and merge with drop of potassium mercuric thiocyanate. White feathery crosses prove zinc.

Stannite $\left(\mathrm{Cu}_{2} \mathrm{~S} \text {.FeS.SnS }\right)_{2}$.-Decompose mineral with 1:1 $\mathrm{HNO}_{3}$. Leach residue with drop of 1:7 $\mathrm{HNO}_{3}$, transfer drop, and merge with drop of potassium mercuric thiocyanate solution. Greenish-yellow mosslike aggregates and isolated prisms prove copper. Test solution turns pink, proving iron. Remove last traces of copper in original residue by leaching it with two successive drops of 1:7 $\mathrm{HNO}_{3}$ and one of $\mathrm{H}_{2} \mathrm{O}$; reject all three drops. Leach residue 
with drop of $1: 5 \mathrm{HCl}$, transfer drop, and add fragment of rubidium chloride. Colorless highly refracting octahedra prove tin.

Sternbergite $\left(\mathrm{AgFe}_{2} \mathrm{~S}_{3-4}\right)$.-Decompose mineral with 1:1 $\mathrm{HNO}_{3}$. Leach residue with 1 per cent $\mathrm{HNO}_{3}$, transfer drop, and add fragment of ammonium bichromate. Red crystals prove silver. Leach original residue with a drop of 1: $7 \mathrm{HNO}_{3}$, transfer drop, and add fragment of $\mathrm{K}_{4} \mathrm{Fe}(\mathrm{CN})_{\text {f. }}$. An amorphous blue precipitate proves iron. Most of the iron was carried over with the silver in the first drop, but there is usually enough left in the original residue to yield a satisfactory test.

Stephanite $\left(5 \mathrm{Ag}_{2} \mathrm{~S}_{2} \mathrm{Sb}_{2} \mathrm{~S}_{3}\right)$.-Decompose mineral with $1: 1 \mathrm{HNO}_{3}$. Leach residue with 1 per cent $\mathrm{HNO}_{3}$, transfer drop, and add fragment of ammonium bichromate. Red crystals prove silver. Leach original residue with a drop of 1:5 $\mathrm{HCl}$, transfer drop, and add fragment of KI. Solution turns yellow near the fragment as it dissolves. Then add a fragment of $\mathrm{CsCl}$. Orange-colored hexagons and stars prove antimony.

Stibnite $\left(\mathrm{Sb}_{2} \mathrm{~S}_{3}\right)$. - The $\mathrm{KOH}$ etch test is very characteristic. The presence of antimony can be confirmed microchemically as follows: Decompose mineral with aqua regia. Leach residue with drop of 1:5 HCl, transfer drop, and add fragment of KI. Solution turns yellow near fragment as it dissolves. Add fragment of $\mathrm{CsCl}$ to solution. Orange-colored stars and hexagons, the stars predominating, prove antimony.

Stromeyerite $\left(\left(\mathrm{Ag}_{2}, \mathrm{Cu}\right) \mathrm{S}\right)$.-Decompose mineral with $1: 1 \mathrm{HNO}_{3}$, leach residue with drop of 1 per cent $\mathrm{HNO}_{3}$, transfer drop, and add fragment of ammonium bichromate. Red crystals prove silver. Leach residue with drop of 1: $7 \mathrm{HNO}_{3}$, transfer drop, and merge with drop of potassium mercuric thiocyanate solution. Greenish-yellow mosslike aggregates and prisms prove copper.

Sulvanite $\left(\mathrm{Cu}_{3} \mathrm{VS}_{4}\right)$.-Decompose mineral with 1:1 $\mathrm{HNO}_{3}$. Mineral is very resistant to chemical attack, and at least 10 successive applications of acid will be necessary. Leach residue with drop of 1:7 $\mathrm{HNO}_{3}$, transfer drop, and merge with drop of potassium mercuric thiocyanate reagent. Greenish-yellow mosslike clumps and prisms prove copper. A satisfactory test for vanadium is not available.

Sylvanite (( $\left.\mathrm{Ag}, \mathrm{Au}) \mathrm{Te}_{2}\right)$.-Decompose mineral with $1: 1 \mathrm{HNO}_{3}$. One or two applications of acid suffice. The residue is a white curdy powder. Leach residue with 1:5 HCl. The white curdy residue goes into solution at once. Transfer drop and add a fragment of $\mathrm{CsCl}$. Honey-yellow isometric crystals prove tellurium. Gold remains in the original residue in small metallic grains and lumps. Usually examination in oblique reflected light with a 16-millimeter objective suffices to identify the gold. If the appearance of the residue is not sufficiently characteristic, add drop of aqua regia to residue and evaporate to dryness. An orange-colored residue of gold chloride remains. Dissolve this in water, transfer drop, and merge with it a drop of pyridine- $\mathrm{HBr}$ reagent. Strongly pleochroic (colorless to deep red) prisms prove gold. Silver remains in the original residue as a curdy white chloride. Leach it with two or three successive drops of 1:5 $\mathrm{HCl}$ to remove remaining traces of gold and tellurium, then one of water to remove $\mathrm{HCl}$. Then add to original residue a drop of concentrated $\mathrm{NH}_{4} \mathrm{OH}$. Observe with an 8-millimeter objective. In a few seconds tiny colorless isometric crystals of $\mathrm{AgCl}$ will be seen floating in the ammonia solution.

Teallite (PbS.SnS).-Decompose mineral with 1:1 $\mathrm{HNO}_{2}$. Leach residue with drop of $1: 5 \mathrm{HCl}$, transfer drop, and add fragment of rubidium chloride. Colorless, highly refracting octahedra prove tin. Add a drop of water to the original residue and evaporate over microflame to volatilize remaining trace 
of HCl. Cool again, add a drop of water to the original residue, transfer drop, and add fragment of KI. Yellow hexagons prove lead.

Tellurium (Te).-Dissolve in 1:1 $\mathrm{HNO}_{3}$. Leach residue with drop of 1:5 $\mathrm{HCl}$, transfer drop, and add fragment of CsCl. Honey-yellow isometric crystals prove tellurium.

Tennantite $\left(5 \mathrm{Cu}_{2} \mathrm{~S} .2(\mathrm{Cu}, \mathrm{Fe}, \mathrm{Zn}) \mathrm{S} .2 \mathrm{As}_{2} \mathrm{~S}_{3}\right)$.-Decompose mineral with $1: 1$ $\mathrm{HNO}_{3}$. Leach residue with 1: $7 \mathrm{HNO}_{3}$, transfer drop, and evaporate slowly over microflame. When nearly dry, add drop of ammonium molybdate solution and again slowly evaporate. When dry, cool and add drop of 1:7 $\mathrm{HNO}_{3}$ to redissolve excess of ammonium molybdate. Tiny yellow isometric crystals prove arsenic. Transfer filtrate and merge with it a drop of potassium mercuric thiocyanate solution. Greenish-yellow mosslike clumps and prisms prove copper.

Tetradymite $\left(\mathrm{Bi}_{2}(\mathrm{Te}, \mathrm{S})_{3}\right)$.-Dissolve mineral in 1:1 $\mathrm{HNO}_{3}$. Dissolve residue in drop of 1:5 HCl. Add fragment of $\mathrm{CsCl}$. Colorless iridescent rhomb-shaped and blade-shaped plates prove bismuth. In addition honey-yellow isometric crystals also form, proving tellurium.

Tetrahedrite $\left(5 \mathrm{Cu}_{2} \mathrm{~S} .2(\mathrm{Cu}, \mathrm{Fe}, \mathrm{Zn}) \mathrm{S} .2 \mathrm{Sb}_{2} \mathrm{~S}_{3}\right)$. -Decompose mineral by means of 1:1 $\mathrm{HNO}_{3}$. Leach residue with drop of 1: $7 \mathrm{HNO}_{3}$, transfer drop, and merge with it a drop of potassium mercuric thiocyanate solution. Greenish-yellow mosslike clumps and prisms prove copper. Leach residue with two successive drops of 1: $7 \mathrm{HNO}_{3}$ to remove copper and reject both drops. Then leach residue with a drop of 1:5 $\mathrm{HCl}$, transfer drop, and add fragment of $\mathrm{CsCl}$. The solution near the fragment turns yellow as the KI dissolves. Then add fragment of CsCl. Orange-colored hexagons and stars prove antimony.

Tiemannite (HgSe).-Decompose mineral with aqua regia. Examine residue in oblique reflected light for mercuric chloride lattice and red tinge of elemental selenium. Leach residue with 1 per cent $\mathrm{HNO}_{3}$, transfer drop, and add a small fragment of cobalt nitrate. When dissolved add a fragment of KCNS. Indigoblue arborescent forms and prisms prove mercury. Leach original residue with drop of 1:5 HCl, transfer drop, and merge with a drop of stannous chloride solution. A brick-red amorphous precipitate proves selenium.

Troilite (FeS).-Dissolve mineral in 1:1 $\mathrm{HNO}_{3}$. Leach residue with drop of 1:5 HCl, transfer drop, and add fragment of $\mathrm{K}_{\mathrm{f}} \mathrm{Fe}(\mathrm{CN})_{6}$. Amorphous blue precipitate proves iron.

Ulmanite (NiSbS).-Decompose mineral with $1: 1 \mathrm{HNO}_{3}$. Leach residue with drop of 1: $7 \mathrm{HNO}_{3}$, transfer drop, and evaporate to dryness. To the residue from this evaporation add a drop of dimethyl glyoxime solution. A pink precipitate proves nickel. Leach original residue with a drop of $1: 5 \mathrm{HCl}$, transfer drop, and add fragment of KI. Solution turns yellow near fragment as it dissolves. Then add fragment of $\mathrm{CsCl}$. Orange-colored hexagons and stars prove antimony.

Umangite $\left(\mathrm{Cu}_{3} \mathrm{Se}_{2}\right)$.-Decompose mineral with 1:1 $\mathrm{HNO}_{3}$. Examine residue in oblique reflected light for a reddish tinge, indicating selenium. Leach residue with a drop of 1 per cent $\mathrm{HNO}_{3}$, transfer drop, and merge with drop of potassium mercuric thiocyanate solution. Greenish-yellow mosslike aggregates and isolated prisms prove copper. Leach original residue with drop of 1:5 $\mathrm{HCl}$, transfer drop, and merge with drop of stannous chloride solution. An amorphous brick-red precipitate proves selenium.

Violarite $\left((\mathrm{Ni}, \mathrm{Fe})_{3} \mathrm{~S}_{4}\right)$. -Decompose mineral with $1: 1 \mathrm{HNO}_{3}$. Leach residue with 1: $7 \mathrm{HNO}_{3}$, transfer drop, and evaporate to dryness. Add drop of 1 per cent $\mathrm{NH}_{4} \mathrm{OH}$ to residue. A yellow gelatinous precipitate of $\mathrm{Fe}(\mathrm{OH})_{3}$ proves iron. Allow the $\mathrm{NH}_{4} \mathrm{OH}$ to evaporate in air. When nearly dry, add directly a drop of dimethyl glyoxime solution. A pink precipitate will form on top of the gelatinous ferric hydroxide coating, proving nickel. 
Voltzite $(4 \mathrm{ZnS} . \mathrm{ZnO})$.-Decompose mineral with aqua regia. Leach residue with drop of 1: $7 \mathrm{HNO}_{3}$, transfer drop, and merge with drop of potassium mercuric thiocyanate. White feathery crosses prove zinc.

Vrbaite $\left(\mathrm{Tl}_{2} \mathrm{~S} .3(\mathrm{As}, \mathrm{Sb})_{2} \mathrm{~S}_{3}\right)$. - Thallium tested for by flame coloration. A small loop at the end of the platinum wire was filled with a drop of $1: 1 \mathrm{HCl}$ and a small fragment of mineral placed in this loop. This was placed in the hottest part of a Bunsen flame. A vivid green color identified thallium. Test for arsenic and antimony microchemically as follows: Decompose mineral with 1:1 $\mathrm{HNO}_{3}$. Leach residue with drop of $1: 7 \mathrm{HNO}_{3}$ and slowly evaporate over microflame. When nearly dry add drop of ammonium molybdate solution and again evaporate slowly over microflame. When dry add a drop of 1:7 HNO. to redissolve excess of ammonium molybdate. Tiny yellow isometric crystals prove arsenic. Leach original residue with drop of 1:5 HCl, transfer drop, and add fragment of $\mathrm{CsCl}$. Colorless hexagons prove antimony.

Weissite (Cu,Te; exact proportions not known).-Take mineral into solution with $1: 1 \mathrm{HNO}_{3}$. Leach residue with 1 per cent $\mathrm{HNO}_{3}$, transfer drop, and merge with drop of potassium mercuric thiocyanate solution. Greenish-yellow mosslike aggregates and prisms prove copper. Leach original residue with 1:5 HCl, transfer drop, and add fragment of $\mathrm{CsCl}$. Honey-yellow isometric crystals prove tellurium.

Willyamite ((Co,Ni)SbS).-Decompose mineral with drop of $1: 1 \mathrm{HNO}_{3}$. Leach residue with drop of 1:7 $\mathrm{HNO}_{2}$, transfer drop, and merge with it a drop of potassium mercuric thiocyanate. If both cobalt and nickel are present, both blue and brown spherulites will form. If cobalt is present in sufficient concentration, blue prisms will also form. (See description on p. 136.) Leach original residue with a drop of 1:5 HCl. Transfer drop and add fragment of KI. The solution turns yellow near the drop as it dissolves. Then add fragment of $\mathrm{CsCl}$. Orange-colored hexagons and stars prove antimony.

Wittichenite $\left(3 \mathrm{Cu}_{2} \mathrm{~S}_{3} \mathrm{Bi}_{2} \mathrm{~S}_{3}\right)$.-Decompose mineral with 1:1 $\mathrm{HNO}_{3}$. Leach residue with drop of $1: 7 \mathrm{HNO}_{3}$, transfer drop, and merge with drop of potassium mercuric thiocyanate solution. Greenish-yellow mosslike clumps and prisms prove copper. Leach original residue with drop of $1: 5 \mathrm{HCl}$, transfer drop, and add fragment of $\mathrm{CsCl}$. Thin colorless rhombohedral plates prove bismuth. The double iodide test for bismuth does not work well, as more or less copper is carried over with the bismuth, and this is precipitated by KI, masking the bismuth test.

Wurtzite $(\mathrm{ZnS})$.-Decompose mineral with aqua regia. Leach residue with drop of 1: $7 \mathrm{HNO}_{3}$, transfer drop, and merge with drop of potassium mercuric thiocyanate reagent. White feathery crosses prove zinc.

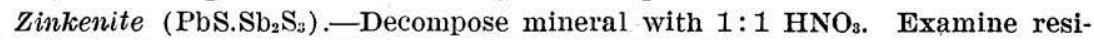
due for lattice structure, indicating lead. Leach residue with drop of 1:5 HCl. Transfer drop and add fragment of KI. Solution turns yellow near fragment as it dissolves. Then add fiagment of cesium chloride. Orange-colored hexagons and stars prove antimony. Add drop of water to original residue and evaporate over flame to remove remaining traces of $\mathrm{HCl}$. Cool and again add drop of water to original residue. Transfer drop and add fragment of KI. Glistening yellow hexagons prove lead. 



\section{INDEX}

\begin{tabular}{lr|} 
& Page \\
\hline &
\end{tabular}

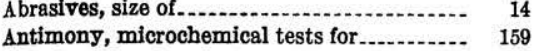

Arsenic, microchemical tests for ............... 155

B

Bakelite

Bateman, A. M. ................. 13, 42 Behrens, H.... 115, 116, 117, 135, 139, 155, 156, 165, 166 Binnenthal minerals........................... 103 Bismuth, microchemical tests for ............ $\quad 159$

Boricky, E........................................ 115

Bottle, glass-stoppered dropping ............. $\quad 128$

Broderick, T. M......................... 71

Busch microscope lamp........................ 42

Buttgenbach, $\mathrm{H}$

\section{$\mathrm{C}$}

Cabeen, C. $\mathbf{K}$

Cadmium, microchemical test for

Camera, Bausch \& Lomb Le Chatelier model. improvised

Leitz "Makam"

Canfleld mineral collection

Capillary tubes.

Chalcography

Chamot, E. M

$117,118,120,121,123,124,125,132,135,139$, $143,144,145,146,155,164,165,169$.

Clennell, J. E

Cobalt, microchemical tests for

Color.

Color standards, Ridgway's.

Colored minerals, table.

Comparison microscope.

Copper, microchemical tests for

Cupferron

\section{D}

Dana, J. D.

Davy, W. M....... 1, 2, 3, 11, 17, 36, 49, 50, 51, 52, 103

Determinative table

Development of photographic plate

Discredited and doubtful minerals

E

Etch reactions

Etch reagents

Exposure formula.

\section{F}

Fahey, J. J

Fairbanks, E. E Farnham, C. M Filters, light_........................................ 33

Flotation of polishing powders
Foshag, W. F

Page

Fraser, H. J

vII, 59,97

Frebold, Georg

VII, 173

104

\section{G}

Gold, microchemical tests for................ 152 Graton, L. C................................ 52

\section{$\mathrm{H}$}

Hardness_.............. 35

Harvey, R. D

Hind, H. L

I

Illuminator, vertical.............................

Impregnation of specimen ..................... 15

Index of minerals, alphabetic................. 61

grouped by elements..................... 107

Internal reflection, table of minerals showing. $\quad 34$

Iron, microchemical tests for............. 139

K

Kerr, P. F._.......... 49, 56

Kley, P. D. C..... 116, 117, 135, 139, 155, 156, 165, 166

Koenigsberger, J................................. 39

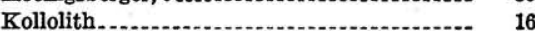

L

Landes, K. K................................ 117

Laney, F. B ...... 76

Lead, microchemical tests for.................. 143

Leveling cup.................................. 7

Lindgren, Waldemar................... 86, 116

Lovering, T. S.................................... VII

M

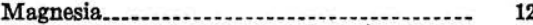

Manganese, microchemical tests for ........... 168

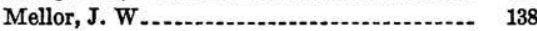

Mercury, microchemical tests for............ 142

Merwin, H. E............... 105

Metallographic microscope................. 3

Microburner, alcohol........................... 121

Microchemical methods...................... $\quad 115$

Mineragraphy................................... 3

Mineralography

Mounts, brass............................. 8

Murdoch, Joseph................ 1, 3, 30, 32, 36, 50, 52

Murdoch ore mineral suite.................. VII, 2, 38

McKinstry, H. E................................ 116, 137

$\mathbf{N}$

Needle, chisel-pointed......- 119

Níckel, microchemical tests for................ 133

Nitroso $\beta$-naphthol 
\begin{tabular}{ll}
0 & Page \\
\hline &
\end{tabular}

Osborne, F. F

Outline of determinative table.

Oven, warming.

\section{P}

Photomicrography of polished sections

Plate, Wratten M panchromatic

Polarized light.

Polishing process at the U. S. Geological Survey.

at Harvard University.

at University of Minnesota.

Potassium mercuric thiocyanate, preparation of

Posnjak, E

Powders, colored, table of minerals with.... $\quad 35$

Powers, H. A. vII, 173

Putnam, P. C - $116,129,153$

\section{$\mathbf{R}$}

Ramdohr, Paul

Randles, W. B

Reagents, etch microchemical.

Reed, F. S

Roberts, E. J

Roebling mineral collection

Ross, C. S

Rouge, black magnetic

Samson cloth. Sampson, Edward

Saw, diamond.

Schneiderhöhn, Hans

Schwartz, G. M

Selchow, D

Selenium, microchemical tests for
Page

Sensitivity of microchemical tests........... 126 Shannon, E. V............. 74, 77, 78, 82, 87, 94, 101

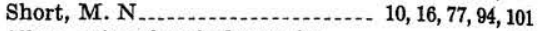
Silver, microchemical tests for ............... 148 Slides, glass . . . Smitheringale, W. V. . . . . 54

Specific tests for minerals.................... 179

Spencer, L. J............................ 91

Steiger, George....... vir

Sulphur, microchemical tests for............. 169

Systematic scheme of microchemical analysis. . 173

$\mathrm{T}$

Talmage hardness machine ............... 37

Talmage, S. B . . . . . . $31,32,35,37$

Tellurium, microchemical tests for ............ 165

Thiel, G. A ........... vII, 54

Thomson, Ellis.................................. 100

Tin, microchemical tests for .............. 163

Tubes, capillary 122

\section{V}

Van der Veen, R. W ......... 1, 5, 37, 40, 51, 52 Vanderwilt, J. W............................ 11,38

W

Walker, T. L._. . .

Wax, modeling .............................. 7 sealing

Wells, R. C.................................... 94

Wherry, E. T.

Whitehead, W. L........................... 3

Winchell, N. H. and A. N.................... 39

Wright biquartz wedge.......... 40, 44, 45, 46

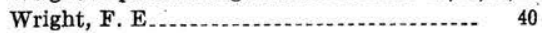

Z

Zinc, microchemical tests for 

